\title{
Fenômenos críticos em sistemas \\ de partículas interagentes \\ e suas aplicações na modelagem \\ de mercados financeiros
}

\section{Fernando Pigeard de Almeida Prado}

TESE APRESENTADA

$\mathrm{AO}$

INSTITUTO DE MATEMÁTICA E ESTATÍSTICA

DA

UNIVERSIDADE DE SÃO PAULO

PARA OBTER O GRAU

$\mathrm{DE}$

DOUTOR EM ESTATÍSTICA

Área de Concentração: Probabilidade

Orientador: Prof. Dr. Vladimir Belitsky

-São Paulo, 27 de janeiro de 2004-

Durante a realização deste trabalho, o autor recebeu suporte financeiro da Fapesp. 


\title{
Fenômenos críticos em sistemas de partículas interagentes \\ e suas aplicações na modelagem de mercados financeiros
}

\author{
Este exemplar corresponde à redação final \\ da dissertação devidamente corrigida e \\ defendida por Fernando Pigeard de Almeida Prado \\ e aprovada pela comissão julgadora.
}

São Paulo, 30 de abril de 2004.

Banca Examinadora:

- Prof. Dr. Vladimir Belitsky (Presidente) - IME - USP

- Prof. Dr. Henrique von Dreifus - IME - USP

- Prof. Dr. Antonio Fernando Ribeiro de Toledo Piza - IF - USP

- Prof. Dr. Liacir dos Santos Lucena - Instituto de Física - UFRN

- Prof. Dr. Ernesto Modecki - Instituto de Matematica - Universidad de la Republica (Uruguai) 


\section{Agradecimentos}

A Vladimir Belitsky pelo estímulo, orientação e aposta em um novo tipo de pesquisa. A Antonio Luiz Pereira pela generosidade e disponibilidade no final do processo. A Pablo Ferrari pela amizade e apoio. A Paolo Tommasini pelas preciosas sugestões.

Ao Marcus, meu sobrinho, por ter me ajudado na elaboração das figuras deste trabalho. À Adriana por toda a ajuda, paciência e carinho. 


\section{Resumo}

Apresentamos um modelo estocástico de agentes interagentes que permite investigar a formação de preço em mercados financeiros como sendo um resultado de dois fatores: a interação social entre agentes e a heterogeneidade de suas crenças individuais. Nós mostramos que nosso modelo exibe transição de fase. Para encontrar as propriedades desta transição, importante para a aplicação, nós analisamos certos sistemas dinâmicos. Tanto a existência de transição de fase quanto os sistemas dinâmicos que emergem deste estudo tornam o modelo interessante do ponto de vista de um estudo matemático. $\mathrm{O}$ modelo também é atraente do ponto de vista de aplicação, pois explica a formação de bolhas especulativas e crashes, assim como a multiplicidade de preços de equilíbrio em mercados financeiros. 


\section{Abstract}

We present a stochastic model of interacting agents that allows one to investigate price formation in financial markets as a result of the combination of two factors: the social interactions between agents and the heterogeneity of their individual beliefs. We show that our model exhibits phase transition. To find the properties of this phase transition, important for application, we analyze certain dynamical systems. Both the existence of phase transition and the dynamical systems that emerge in this study, make our model interesting from the point of view of mathematical study. The model is also attractive from the point of view of application. The model explains formation of bubbles and crashes, as well as multiplicity of equilibrium prices in financial markets. 


\section{Sumário}

1 Introdução 1

1.1 Revisão bibliográfica comentada . . . . . . . . . . . . . . . . . . . 2

1.2 Modelos de agentes heterogêneos interagentes . . . . . . . . . . . . . 5

1.3 Objeto da tese . . . . . . . . . . . . . . . . . . . . . . 6

1.4 Apresentação do trabalho de tese e resumo dos principais resultados . . . . 7

2 Modelo de Influência Social Determinística $\quad 9$

2.1 Definição do modelo . . . . . . . . . . . . . . . . . . . . . 9

2.1.1 Excesso de demanda relativo . . . . . . . . . . . . . . . . . 9

2.1 .2 Ajustamento do preço . . . . . . . . . . . . . . . . 10

2.1.3 Heterogeneidade das avaliações individuais sobre o valor fundamental do ativo . . . . . . . . . . . . . . . . . . 10

2.1.4 Fundamentalistas e especuladores . . . . . . . . . . . . . . . 12

2.1.4.1 Regra de decisão fundamentalista . . . . . . . . . . . . . . 12

2.1.4.2 Regra de decisão especulativa . . . . . . . . . . . . . 13

2.2 Processo da sobrevalorização do ativo e excesso de demanda relativo . . . . 14

2.3 Limite do processo para grandes populações de agentes (Proposição 1) . . . 17

2.3.1 Corolários da Proposição 1 . . . . . . . . . . . . . . . . . 21

2.3.2 Demonstração da Proposição 1 . . . . . . . . . . . . . . . . . 24

2.4 Dinâmica de grandes populações . . . . . . . . . . . . . . . . . . . . . . 32

2.4.1 Caso $J \geq 0, K=0$ e $\lambda=0\left(H_{t}=h\right.$ não varia no tempo $) \ldots . .33$

2.4.1.1 Justificativa do interesse no caso $\lambda=0 \ldots . . . . .34$

2.4.1.2 Dinâmica de excesso de demanda $\left(\bar{A}_{t}\right)_{t \geq 0} \ldots \ldots$. . . . . 36

2.4.1.3 Excesso de demanda de equilíbrio . . . . . . . . . . . . 36 
2.4.1.4 O resultado principal: transição de fase entre excessos de demanda de equilíbrio . . . . . . . . . . . 38

2.4.1.5 Interpretações socioeconômicas: estabilidade global do excesso de demanda ou formação exógena de bolhas especulativas e crashes . . . . . . . . . . . . . . . . . 44

2.4.1.6 Similaridade com o modelo de Ising ferromagnético com interação de longo alcance . . . . . . . . . . . . . . . . 48

2.4.1.7 Melhoramento da estimativa de convergência do processo estocástico para o sistema dinâmico determinístico . . . . 51

2.4.2 Caso $J \geq 0, K=0$ e $\lambda>0$ ( $H_{t}$ varia no tempo $) \ldots \ldots \ldots \ldots 5$

2.4.2.1 Dinâmica da sobrevalorização e excesso de demanda do ativo $\left(H_{t}, \bar{A}_{t}\right)_{t \geq 0} \ldots \ldots \ldots \ldots \ldots \ldots$

2.4.2.2 Equilíbrios de $\left(H_{t}, \bar{A}_{t}\right)_{t \geq 0} \ldots \ldots \ldots \ldots \ldots \ldots$

2.4.2.3 O resultado principal: transição de fase entre existência de um único equilíbrio globalmente estável de $\left(H_{t}, \bar{A}_{t}\right)_{t \geq 0}$ em $(0,0)$ e comportamento cíclico de $\left(H_{t}, \bar{A}_{t}\right)_{t \geq 0} \quad \ldots .56$

2.4.2.4 Interpretação socioeconômica: estabilidade do preço de equilíbrio de mercado ou formação endógena de bolhas especulativas e crashes de preço . . . . . . . . . . 59

2.4.2.5 Melhoramento da estimativa de convergência do processo estocástico para o sistema dinâmico determinístico . . . . 62

2.4.3 Caso $J=0, K \geq 0$ e $\lambda=0$ ( $H_{t}=h$ não varia no tempo $) \ldots . .64$

2.4.3.1 Dinâmica de excesso de demanda $\left(\bar{A}_{t}\right)_{t \geq 0} \ldots \ldots \ldots 64$

2.4.3.2 Excesso de demanda de equilíbrio . . . . . . . . . . 65

2.4.3.3 Transição de fase entre excessos de demanda de equilíbrio 66

2.4.3.4 O resultado principal: negatividade do atrator superior . . 67

2.4.4 Caso $J=0, K \geq 0$ e $\lambda>0$ ( $H_{t}$ varia no tempo $\ldots \ldots \ldots \ldots$

2.4.4.1 Dinâmica da sobrevalorização e excesso de demanda do ativo $\left(H_{t}, \bar{A}_{t}\right)_{t \geq 0} \ldots \ldots \ldots \ldots \ldots \ldots \ldots$ 
2.4.4.2 O resultado principal: transição de fase entre existência de dois equilíbrios estáveis de $\left(H_{t}, \bar{A}_{t}\right)_{t \geq 0}$ e comportamento cíclico de $\left(H_{t}, \bar{A}_{t}\right)_{t \geq 0} \ldots \ldots \ldots \ldots \ldots$. . . . . . . . . 71

2.4.4.3 Interpretação socioeconômica: estabilidade local de dois preços de equilíbrio de mercado . . . . . . . . . . . . 72

2.4.4.4 Simulação . . . . . . . . . . . . . . . . . 78

2.4.5 J $\geq 0, K \geq 0, \lambda>0$ (caso geral). Principais resultados . . . . . . 82

2.4.5.1 Principais diferenças entre o caso geral $J \geq 0, K \geq 0$ e os casos $J \geq 0, K=0$ e $J=0, K \geq 0 \quad \ldots . . . . .84$

3 Modelo de Influência Social Estocástica $\quad 85$

3.1 Definição do modelo . . . . . . . . . . . . . . . . . . . . . 85

3.1 .1 Regras de decisão . . . . . . . . . . . . . . . . . . . 85

3.1.2 Heterogeneidade das influências sociais $J_{t}^{(s)}$ e $K_{t}^{(s)}, s \in S, t \geq 0 \quad$. . 86

3.1 .3 Justificativa para a substituição de $P_{t}^{(s)}$ por $\bar{P} \ldots$. . . . . . . . . 86

3.2 Convergência de $\left(W_{t}\right)_{t \geq 0}$ para $\left(w_{t}\right)_{t \geq 0} \ldots \ldots \ldots \ldots$. . . . . . 87

3.2.1 Convergência de $\left(W_{t}\right)_{t \geq 0}$ para $\left(w_{t}\right)_{t \geq 0}$. Caso $J_{t}^{(s)}>0$ e $K_{t}^{(s)}=0$, $s \in S, t \geq 0 \ldots \ldots \ldots \ldots$. . . . . . . . . . . . . . . . . . . . . . . . .

3.2.2 Convergência de $\left(W_{t}\right)_{t \geq 0}$ para $\left(w_{t}\right)_{t \geq 0}$. Caso $J_{t}^{(s)}=0$ e $K_{t}^{(s)}>0$, $s \in S, t \geq 0 \ldots \ldots \ldots \ldots$. . . . . . . . . . . . . . . . . . . .

3.3 Caso $J_{t}^{(s)}>0, K_{t}^{(s)}=0, \forall s \in S, \forall t \geq 0 \ldots \ldots \ldots \ldots$. . . . . . . 92

3.3.1 Dinâmica da sobrevalorização e excesso de demanda do ativo $\left(H_{t}, \bar{A}_{t}\right)_{t \geq 0} \ldots \ldots \ldots$. . . . . . . . . . . . . . . . . . .

3.3.2 O resultado principal: comportamento cíclico de $\left(H_{t}, \bar{A}_{t}\right)_{t \geq 0} \quad \ldots .95$

3.4 Caso $J_{t}^{(s)}=0, K_{t}^{(s)}>0, \forall s \in S, \forall t \geq 0 \ldots \ldots \ldots$. . . . . . . 97

3.4.1 Dinâmica da sobrevalorização e excesso de demanda do ativo $\left(H_{t}, \bar{A}_{t}\right)_{t \geq 0} \ldots \ldots \ldots \ldots$. . . . . . . . . . . . . 97

3.4.2 O resultado principal: transição de fase entre existência de dois equilíbrios estáveis de $\left(H_{t}, \bar{A}_{t}\right)_{t \geq 0}$ e comportamento cíclico de $\left(H_{t}, \bar{A}_{t}\right)_{t \geq 0} 98$

4 Modelo de Influência Social Determinística com Interação Local entre Especuladores 
4.1 Definição do modelo . . . . . . . . . . . . . . . . . . . . . . . . . . 101

4.1.1 Atitude de investimento e excesso de demanda relativo em tempo contínuo . . . . . . . . . . . . . . . . . 101

4.1.2 Ajustamento do preço em tempos exponenciais . . . . . . . . . 102

4.1.3 Tempos exponenciais de atualização de atitude de investimento . 103

4.1.4 Heterogeneidade das avaliações individuais sobre o valor fundamental do ativo (forma paramétrica de $\Phi$ ) . . . . . . . . . . . . 103

4.1.5 Fundamentalistas, especuladores e a rede de influências . . . . . . 104

4.1.6 Regra de decisão fundamentalista . . . . . . . . . . . . . . 105

4.1.7 Regra de decisão especulativa sob influência social local . . . . . . . 105

4.1 .8 Condição inicial . . . . . . . . . . . . . . . . . . . . . . . . 106

4.2 Processo da sobrevalorização do ativo, configuração de atitudes de investimento de especula-dores e média de fundamentalistas . . . . . . . . . 106

4.3 Processo da sobrevalorização e excesso de demanda do ativo $\left(H_{t}, \bar{A}_{t}\right)_{t \geq 0} \ldots 107$ 4.3.1 Caso $J=0, K \geq 0$ e $\lambda=0$ ( $H_{t}=h$ não varia no tempo $) \ldots \ldots 110$ 4.3.1.1 Formalização do processo estocástico a ser estudado e definições dos principais conceitos . . . . . . . . . . . . 110

4.3.1.2 Descontinuidade do excesso de demanda relativo . . . . . 112

4.3.1.3 Histerese . . . . . . . . . . . . . . . . . . . . 113

4.3.1.4 Sobrevalorização crítica do ativo $h_{0} \ldots \ldots \ldots \ldots \ldots$

4.3.2 Caso $J=0, K \geq 0$ e $\lambda>0$ ( $H_{t}$ varia no tempo $\ldots \ldots \ldots \ldots 117$

4.3.2.1 Embasamento da conjectura e a escolha apropriada dos tempos de atualização do preço . . . . . . . . . . . . . 119

4.3 .3 Observações finais . . . . . . . . . . . . . . . . . . . . . . . . 124

5 Conclusão 126

6 Apêndice 129

6.1 Demonstração da Proposição 2 . . . . . . . . . . . . . . . . . . . . . 129

6.2 Demonstração da Proposição 4 . . . . . . . . . . . . . . . . . . . . . . 142

6.2.1 Demonstração no caso $\left(\Phi^{\prime}(0)\right)^{-1}>2 \alpha J \quad \ldots \ldots \ldots . . . . . . .143$

6.2.2 Demonstração no caso $\left(\Phi^{\prime}(0)\right)^{-1}<2 \alpha J \ldots \ldots . . \ldots . . . . .152$ 


\section{Capítulo 1}

\section{Introdução}

Em 1995, T. Lux [19] propôs um modelo para a explicação de bolhas especulativas e crashes de preços no mercado de ações. Neste modelo, T. Lux sugere que agentes econômicos se deixem influenciar pela atitude de investimento de outros agentes. Partindo de um mecanismo de contágio de opiniões, T. Lux deriva a dinâmica do prę̧o. Esta abordagem vai contra a "hipótese de eficiência de mercado" (HEM). Sua contestação à HEM tem como base o trabalho de West, publicado em 1988 [31]. Neste artigo, West conclui que a variação da volatilidade de uma ação observada ao longo do tempo não pode ser adequadamente explicada por modelos baseados no passeio aleatório (com incrementos homogêneos no tempo). West sugere então a necessidade de se considerar modelos que foquem em mecanismos sociológicos e psicológicos que venham a capturar forças intrínsecas à dinâmica do preço.

Mais recentemente, vários modelos e resultados oriundos da teoria de Sistemas de Partículas Interagentes vêm sendo aplicados ao desenvolvimento de modelos em economia e finanças, a fim de contemplar interdependências sociais que possam ter um impacto em variáveis socioeconômicas. Estas variáveis são, por exemplo, a demanda por um ativo financeiro, o desemprego, a criminalidade e a produtividade de um determinado setor industrial. Nestes modelos, são tipicamente postuladas as interdependências individuais do ponto de vista socioeconômico e, posteriormente, são analisados os comportamentos macroscópicos emergentes destas interações, mediante as técnicas já desenvolvidas para o estudo de Sistemas de Partículas Interagentes (SPI). 


\subsection{Revisão bibliográfica comentada}

Apresentaremos a seguir uma revisão bibliográfica comentada de grande parte dos trabalhos já desenvolvidos na área de economia e finanças que se utilizam de técnicas em SPI. Boa parte desta revisão advém do artigo de J. A. Scheinkman, de 2000 [23], e de outros trabalhos que serviram de consulta para a elaboração do presente trabalho.

1. O primeiro artigo em economia a usar este tipo de estrutura foi escrito por Föllmer [1974] [11]. Em seu artigo, ele modelou uma economia cuja estrutura de preferências dos agentes apresenta uma interdependência local. Com base na estrutura do modelo de Ising, ele examinou quando uma aleatoriedade nas preferências dos agentes pode afetar o comportamento macroscópico resultante, mesmo quando o número de agentes tende para o infinito (limite termodinâmico). Como resultado, obteve dois vetores de preços de mercado possíveis; cada qual correspondendo a uma fase da economia.

2. Outros modelos inspirados em mecânica estatística partem de uma descrição mais explícita de como as decisões individuais são tomadas no tempo. Bak, Chen, Sheinkman e Woodford [1993] [32] estudaram o impacto de choques setoriais independentes sobre a flutuação agregada com um modelo que exibe uma criticalidade auto-induzida.

3. Brock [1993] [6] e Blume [1993] [5] reconheceram a relação entre uma classe de modelos de partículas interagentes e os modelos de escolhas discretas na presença de influências sociais tratados na literatura econômica.

4. Brock e Durlauf [1995] [8]e [9] desenvolveram vários resultados em escolhas discretas na presença de influências sociais.

5. Durlauf [1993] [7] construiu um modelo baseado em interações locais de tecnologia a fim de examinar a possibilidade de entraves na produtividade global, segundo o qual técnicas de baixa produtividade são empregadas justamente porque outros produtores também usam processos de baixa produtividade. 
6. Outros artigos exploram a possibilidade do "processo de aprendizagem dos agentes" . Nestes modelos, os agentes baseiam suas decisões no comportamento de outros agentes: Arthur [1989] [2]; Banerjee [1992] [3]; Bickchandani, Hirshleifer e Welch [1992] [30]; Ellison [1993] [10]; Kirman [1993] [16]; Young [1993] [35]; Ellison e Fudemberg [1993] [12]; e Gul e Lundholm [1995] [18].

7. T. Lux [1995] [19] apresentou um modelo de contágio de opiniões para a descrição da dinâmica de preço em mercados financeiros. Bolhas especulativas e crashes de preço emergem em seu modelo como fenômenos cíclicos.

8. O trabalho de Pesendorfer [1995] [21] sobre os ciclos da indústria da moda examina como um monopolista explora a presença de interações sociais.

9. Topa [1997] [29] examinou a distribuição espacial do desemprego com a ajuda do "processo de contato".

10. Glaeser, Sacerdote e Scheinkman [1996] [22] usaram o "modelo votante" para analisar a distribuição da criminalidade entre as cidades americanas.

11. Glaeser e Scheinkman [2001] [24] baseiam-se no modelo acima proposto usando um modelo de ações contínuas e sugerem uma metodologia para quantificação de interações locais e globais usando a variação de agregados entre cidades.

12. Madrigal e Scheinkman [1997] [25] apresentaram uma explicação para crashes de preço no mercado de ações usando um modelo de ordens de compra e venda ("Bid" e "Ask"). Eles mostraram que o preço de equilíbrio, como uma função do fluxo de ordens, apresenta uma descontinuidade. Esta descontinuidade pode ser interpretada como um crash de preço.

13. Kaizoji [2000] [15], baseado no modelo de Ising de longo alcance, elaborou um modelo para a explicação de bolhas especulativas e crashes como um fenômeno de transição de fase. Com base neste modelo, ele propôs uma explicação qualitativa e quantitativa para a formação de bolha especulativa e subseqüente crash da bolsa de Tóquio, ocorridos entre 1987 e 1992. 
14. Becker e Murphy [2000] [20] apresentaram uma análise abrangente das interações sociais na economia. Este volume estende a análise anterior de Becker (1991) relativa à formação de preços nos restaurantes quando existe interação social na demanda por um restaurante específico.

15. Glaeser e Scheinkman [2000] [23] exploraram a estrutura matemática comum de alguns modelos acima descritos. Eles partiram de um modelo de interação social bastante genérico e estudaram as implicações deste modelo em diversos graus de generalidades. Muitos dos modelos já mencionados emergem como casos especiais da formalização aqui proposta.

16. Scheinkman e Xiong [2002] [33] apresentaram um modelo para a explicação de crashes de preço de mercado (exemplarmente observado no crash das ações de Internet), onde cada agente superestima a precisão de sua informação em relação à informação dos demais agentes.

17. Challet e Zhang [1997] [40], inspirados no problema do "Bar El Farol" enunciado por Arthur [1994] [1], propuseram o "Minority Game" (usaremos a notação MG em referência ao modelo "Minority Game"). Segundo este jogo, cada agente $i \in$ $\{1,2, \ldots, n\}$ de um conjunto de $n$ agentes deve optar por um entre dois lados $a_{t}^{i} \in$ $\{-1,+1\}$ a cada instante de tempo $t \in\{0,1,2, \ldots\}$. A cada instante de tempo $t$, ganham aqueles que optarem pelo lado da minoria. Para a tomada de decisão cada agente observa os últimos $m$ resultados em termos de lados vencedores (por exemplo: $(-1,+1,+1, \ldots-1))$ e, com base em uma estratégia, dada por uma função $f:\{-1,+1\}^{m} \rightarrow\{-1,+1\}$, opta correspondentemente por -1 ou +1 . Cada participante $i$ possui um conjunto $\left\{f^{(i, 1)}, f^{(i, 2)}, \ldots, f^{(i, s)}\right\}$ de $s$ estratégias (funções) escolhidas aleatoriamente do conjunto de todas as estratégias possíveis (conjunto de todas as funções de $\left.f:\{-1,+1\}^{m} \rightarrow\{-1,+1\}\right)$. Cada estratégia $f$ do conjunto de todas as estratégias de todos os agentes recebe uma nota acumulativa $N_{t}(f)$ a cada instante de tempo $t$. Se a estratégia $f$ foi capaz de acertar o lado da minoria no instante $t$ (independentemente se ela foi aplicada ou não), ela recebe a nota $N_{t}(f)=N_{t-1}(f)+1$, caso contrário, $N_{t}(f)=N_{t-1}(f)+0$. Se $f_{t}^{i}(\cdot)$ denota a estratégia do agente $i$, a qual obteve a maior nota até o instante de tempo $t$, então 
sua ação $a_{t}^{i}$ adotada neste instante será $a_{t}^{i}=f_{t}^{i}\left(x_{t-1}, x_{t-2}, \ldots, x_{t-m}\right)$, onde $x_{t-j} \in$ $\{-1,+1\}(j=1,2, \ldots, m)$ denotam os últimos $m$ lados vencedores observados. Apesar de sua simples formulação, o MG apresenta resultados bem interessantes: quando o número total de estratégias $n * s$ ultrapassa $2^{m+1}$, estratégias positivamente correlacionadas (que na maior parte do campo de definição $\{-1,+1\}^{m}$ coincidem) começam a surgir expressivamente. Com isso, um número grande de agentes acaba optando pela mesma ação, resultando em grandes discrepâncias entre o número de perdedores e ganhadores ao longo do tempo. Originariamente, o MG foi estudado através de simulações numéricas. Posteriormente, as evidências destas simulações foram tratadas com maior rigor através de um modelo de spin glas por Challet e Marsilli [1999] [41], Challet, Marsili e Zecchina [2000] [37] e Marsili, Challet e Zecchina [2000] [38] (veja também [36] indicado por Zhang e os artigos na web page www.unifr.ch/econophysics $\rightarrow$ "RESOURCES/minority game" $\rightarrow$ "Analytic results"). O interesse por este modelo cresceu de tal forma que atualmente mais de cem artigos relativos ao modelo compõem a web page www.unifr.ch/econophysics $\rightarrow$ "RESOURCES/minority game".

18. Outros modelos exploram processos de formação de opinião através de um modelo de Ising, onde, diferentemente do reticulado $\mathbb{Z}^{d}$, a estrutura de interações entre os sítios é aleatória e reflete um comportamento social mais natural: "novos" sítios tem maior probabilidade de se conectarem a sítios com maior número de conexões já existentes. Nesta linha, Kacperski e Holyst [1999] [13], Kacperski e Holyst [2000] [14] e Aleksiejuk, Holyst e Stauffer [2002] [28] apresentam algumas contribuições.

\subsection{Modelos de agentes heterogêneos interagentes}

Como havíamos comentado, vários resultados oriundos da teoria de Sistemas de Partículas Interagentes (SPI) vêm sendo aplicados para o desenvolvimento de modelos em economia e finanças a fim de contemplar interdependências sociais que possam ter um impacto no agregado socioeconômico emergente.

Ocorre, no entanto, que uma das características básicas dos SPIs não tem interpretação socioeconômica imediata. Esta característica é a aleatoriedade com que cada partícula 
reage a seu meio. Supondo racionalidade das decisões dos agentes econômicos, tal aleatoriedade não se justifica de imediato ou, pelo menos, torna o modelo pouco inteligível do ponto de vista socioeconômico.

Nos modelos que surgem desta linha de pesquisa, tal aleatoriedade vem sendo tratada essencialmente por dois tipos de abordagem: de um lado, estão os modelos que atribuem esta aleatoriedade a um certo grau de irracionalidade no processo decisório real de cada agente; e, de outro lado, estão os modelos em que esta aleatoriedade é atribuída a diferenças individuais entre os agentes. Estas diferenças individuais dizem respeito a variáveis socioeconômicas específicas e relevantes para a investigação de interesse. Tais variáveis podem ser, por exemplo, a renda, a liquidez, o capital, a avaliação sobre o valor fundamental de um ativo etc. Recentemente, estes modelos têm sido denominados de "modelos de agentes heterogêneos interagentes" (MAHI). Em 2002, E. Glaeser e J. A. Scheinkman [23] publicaram um artigo revisando boa parte da pesquisa desenvolvida nesta segunda ramificação. Neste artigo, eles exploram a estrutura matemática comum de muitos modelos tipo MAHI propostos até então. Os autores partiram de um modelo de interação social bastante genérico e estudaram suas implicações em diversos graus de generalidades.

A construção e análise de modelos tipo MAHI abrem a perspectiva de que certos fenômenos socioeconômicos críticos, como a ocorrência de bolhas especulativas e crash de preço no mercado financeiro, possam ser explicados e quantificados em função da interação social de agentes heterogêneos. O interessante desta abordagem é que essa heterogeneidade pode ser observada na distribuição de probabilidades de certas variáveis socioeconômicas da população de agentes em questão. Este fato facilita o ajuste do modelo à realidade.

\subsection{Objeto da tese}

Em nosso trabalho, apresentamos três modelos para a descrição da dinâmica de preço de um ativo negociado no mercado financeiro (exemplos de ativos financeiros são: ações de uma empresa, títulos de dívida do governo, uma moeda etc.). Os modelos que apresentaremos são do tipo MAHI, i.e., baseados na interação de agentes econômicos heterogêneos. Para a construção desses modelos, desenvolvemos três sistemas de partículas interagentes. 
Uma componente importante do primeiro e do terceiro modelo está na heterogeneidade dos agentes financeiros com relação a suas avaliações individuais sobre o valor fundamental do ativo. O valor fundamental de um ativo é dado pelo preço futuro do ativo estimado no longo prazo e trazido ao valor presente pela taxa de juros acrescida de uma adicional de risco. O segundo modelo introduz o fato de que agentes financeiros diferem uns dos outros com relação à intensidade com que estes são influenciados pela opinião de outros agentes. No primeiro e no segundo modelo, a interação entre as partículas do sistema de partículas interagentes associado ao modelo é de longo alcance. O último modelo é a versão do primeiro modelo, onde as interações entre as partículas do SPI correspondente são locais. Estas interações locais modelam as interações sociais entre os agentes financeiros e seus grupos locais de referência.

Com esses três modelos, propomos uma explicação alternativa tanto qualitativa como quantitativa para a existência de mais de um preço de equilíbrio localmente estável do ativo (igualando oferta e procura), bem como uma explicação para as ocorrências de bolhas especulativas e crashes de preço do ativo.

\subsection{Apresentação do trabalho de tese e resumo dos principais resultados}

Este trabalho está dividido em cinco capítulos e um apêndice.

No Capítulo 2 apresentaremos o primeiro modelo. Neste capítulo, mostraremos também que o processo estocástico do preço, que resulta da definição do modelo, converge para um sistema dinâmico determinístico. Estudaremos o sistema dinâmico de preço para o qual o correspondente processo estocástico de preço converge, e derivaremos essencialmente os seguintes resultados para a dinâmica do preço: se a dispersão das avaliações individuais sobre o valor fundamental do ativo (modeladas por variáveis aleatórias independentes e identicamente distribuídas) for alta, então o preço (no sistema determinístico) converge para o valor esperado dessas avaliações individuais (opinião média sobre o valor fundamental do ativo). Se essa dispersão estiver abaixo de uma dispersão crítica, o preço apresenta um comportamento cíclico em torno do valor esperado das avaliações individuais. Para este comportamento cíclico derivaremos as estimativas sobre os respectivos preços de reversão de tendência (preço de crash). Um outro cenário se estabelece quando variamos a fração 
dos dois tipos de agentes que estaremos considerando nos três modelos: especuladores e fundamentalistas. Se a fração de especuladores for baixa, mas a força de interação entre especuladores, alta, obteremos dois preços de equilíbrio de mercado localmente estáveis que diferem da avaliação média (esperança matemática das avaliações individuais) sobre o valor fundamental do ativo. Um destes preços de equilíbrio de mercado está acima, e o outro, abaixo da avaliação média sobre o valor fundamental do ativo.

No Capítulo 3 apresentaremos o segundo modelo. Neste modelo, cada agente é influenciado pela atitude de investimento de outros agentes, porém, com intensidades diferentes. Estas intensidades de influência correspondem à força de interação entre as partículas do sistema de partículas interagentes correspondente ao modelo. Assumiremos que estas intensidades são distribuídas independentemente sobre a população de especuladores segundo uma distribuição de probabilidades comum para todos os especuladores. Mostraremos que este modelo gera resultados similares ao modelo apresentado no Capítulo 2, porém, neste caso, não haverá uma dispersão crítica das avaliações individuais sobre o valor fundamental do ativo.

No Capítulo 4 apresentaremos o terceiro modelo, que é a versão do primeiro modelo com interações locais entre agentes. Neste modelo, partiremos de uma rede local de influências entre agentes correspondendo a um subconjunto de $\mathbb{Z}^{2}$. Derivaremos algumas propriedades que possibilitaram conjeturar que este modelo se comporta de forma análoga ao primeiro modelo. Neste modelo, introduziremos o conceito de preço metaestável de equilíbrio de mercado, que conjecturamos existir sob condições análogas àquelas exigidas para a existência de preços de equilíbrio de mercado localmente estáveis no contexto do primeiro modelo.

No Capítulo 5 apresentaremos a conclusão deste trabalho, bem como uma investigação pretendida.

No Apêndice apresentaremos as demonstrações de duas proposições. 


\section{Capítulo 2}

\section{Modelo de Influência Social Determinística}

\subsection{Definição do modelo}

\subsubsection{Excesso de demanda relativo}

A cada instante $t=0,1,2, \ldots$, cada agente de um conjunto de $N$ agentes, denotado por $A$, demanda ou oferta uma quantidade $q$ fixa de unidades de um ativo financeiro. A atitude de investimento do agente $i \in A$ no tempo $t$ será denotada por $X_{t}^{(i)}$ seguindo a seguinte convenção:

$$
X_{t}^{(i)}:= \begin{cases}+1 & \text { agente } i \text { demanda } q \text { unidades do ativo no tempo } t \\ -1 & \text { agente } i \text { oferta } q \text { unidades do ativo no tempo } t\end{cases}
$$

Introduzimos

$$
\bar{A}_{t}=\sum_{i \in A} X_{t}^{(i)} / N
$$

De acordo com a convenção estabelecida em (2.1), a diferença entre demanda e oferta do ativo, $q \sum_{i \in A} X_{t}^{(i)}$, dividida pelo volume total de demanda e oferta do ativo, $q \sum_{i \in A}\left|X_{t}^{(i)}\right|$, é dada pela média aritmética das atitudes de investimento de todos os agentes $\bar{A}_{t}$ dada em (2.2). Chamaremos $\bar{A}_{t}$ de excesso de demanda relativo ou simplesmente excesso de demanda, ou, ainda, de média empírica das atitudes dos agentes. 


\subsubsection{Ajustamento do preço}

A cada instante de tempo $t$, um agente especial do modelo, que chamaremos de market maker, ${ }^{1}$ ajusta o preço do ativo pela seguinte regra:

$$
P_{t+1}=P_{t}+\lambda \bar{A}_{t} \text { for } t=0,1,2, \ldots \text { para um dado } \lambda>0
$$

onde $P_{t}$ denota o logaritmo natural do preço do ativo no instante $t$.

Com (2.3) assumimos que o preço seja atualizado por um fator que cresce exponencialmente em função do excesso de demanda relativo. ${ }^{2}$ Notamos que o símbolo $P_{t}$ é tradicionalmente usado para designar o "preço no tempo $t$ ", e, conseqüentemente, $\ln P_{t}$ para designar o logaritmo natural do preço do ativo no tempo $t$. Apesar disso, com um certo abuso de notação e linguagem, optamos por denotar o logaritmo do preço no instante $t \operatorname{com} P_{t}$ e nos referir a $P_{t}$ com a expressão "preço do ativo". Observamos que a constante positiva $\lambda$ é um parâmetro do modelo que regula a reação do preço ao excesso de demanda relativo. Notamos ainda que o logaritmo do quociente entre os preços que o ativo assume em dois instantes subseqüentes de tempo, dado por $P_{t+1}-P_{t}$, é chamado tradicionalmente de retorno do ativo na literatura financeira. Chamaremos $P_{t+1}-P_{t}$ de retorno do ativo ou, com um certo abuso de linguagem, de incremento de preço do ativo.

\subsubsection{Heterogeneidade das avaliações individuais sobre o valor fundamental do ativo}

Um dos fatores que influenciam as decisões de agentes financeiros com relação à compra ou à venda de um ativo é seu "valor fundamental". O valor fundamental de um ativo corresponde aos rendimentos futuros esperados do ativo trazidos ao valor presente por uma taxa de juros acrescida de um adicional de risco.

Um pressuposto essencial do modelo é que não existe um único valor fundamental do ativo. Isto porque assumimos que cada agente financeiro tem uma expectativa específica sobre os rendimentos futuros do ativo, bem como uma avaliação individual sobre os riscos de insolvência da contraparte. Portanto, partimos simplesmente da existência

\footnotetext{
${ }^{1}$ A suposição da existência de um market maker pode ser encontrada também nas contribuições de $\mathrm{T}$. Lux [19] e T. Kaizoji [15].

${ }^{2}$ Essa estrutura pode ser encontrada também no artigo de D. Challet, M. Marsili e Y.-C.Zhang [34].
} 
de "avaliações individuais (específicas de cada agente) sobre o valor fundamental do ativo". Estas avaliações individuais têm a dimensão do preço do ativo. Por questões de conveniência, denotaremos com $P_{t}^{(i)}$ o logaritmo da avaliação individual sobre o valor fundamental do ativo feita pelo agente $i$ no instante $t$ (observe que $P_{t}^{(i)}$ tem a mesma dimensão de $P_{t}$ apresentado em (2.3)). Referiremo-nos a $P_{t}^{(i)}, i \in A$, simplesmente como sendo as avaliações individuais sobre o valor fundamental do ativo no instante $t$.

A fim de admitir uma heterogeneidade entre avaliações individuais sobre o valor fundamental do ativo a cada instante de tempo $t$, modelamos as mesmas como sendo variáveis aleatórias independentes e identicamente distribuídas, assumindo a seguinte estrutura:

$$
P_{t}^{(i)}=\bar{P}+\theta_{t}^{(i)} \quad\left(\theta_{t}^{(i)} \sim \Phi\right) \quad i \in A
$$

onde $\bar{P}$ denota a avaliação média (valor esperado) das avaliações individuais sobre o valor fundamental do ativo e $\theta_{t}^{(i)}, i \in A, t=0,1, \ldots$, denotam variáveis aleatórias independentes e identicamente distribuídas, ${ }^{3}$ cuja função de distribuição de probabilidades acumulada será denotada por $\Phi$. Assumimos que $\Phi$ seja diferenciável em todo $\mathbb{R}$. Denotamos por $\Phi^{\prime}$ a derivada de $\Phi$. Assumiremos também que $\Phi^{\prime}$ é contínua em $\mathbb{R}$ e satisfaz as seguintes propriedades:

1) Simetria: $\quad \Phi^{\prime}(x)=\Phi^{\prime}(|x|) \quad \forall x \in \mathbb{R} \quad$ e

2) Monotonicidade: $\Phi^{\prime}$ é estritamente decrescente em $[0, \infty)$

Pelas propriedades dadas em (2.5), segue que a avaliação média sobre o valor fundamental do ativo é a esperança matemática de $P_{t}^{(i)}$, i.e., $\bar{P}=\mathbb{E}\left(P_{t}^{(i)}\right)$.

Estudaremos, entre outros fenômenos, como uma variação (abrupta) de $\bar{P}$ pode alterar o regime da dinâmica $\left(\bar{A}_{t}\right)_{t \geq 0}$ do excesso de demanda relativo do ativo. Entendemos que uma variação de $\bar{P}$ (avaliação média sobre o valor fundamental do ativo) ocorre na vida real devido a notícias que bombardeiam o mercado de tempos em tempos. Essas notícias

\footnotetext{
${ }^{3}$ Apesar de a suposição de independência no tempo das variáveis $\theta_{t}^{(i)}, t=0,1, \ldots,(i \in A)$ não ser muito realista (em geral, investidores otimistas tendem a permanecer otimistas e vice-versa), ela facilita a exposição dos principais fatos que queremos expor. Notamos, no entanto, que esta suposição não é necessária no caso do modelo apresentado na presente seção (modelo de campo médio), ou seja, obteríamos os mesmos resultados se escolhêssemos uma única vez $\theta^{(i)}, i \in A$, independentes e identicamente distribuídas para todos os tempos e congelássemos as avaliações individuais sobre o valor fundamental do ativo, tais que $P_{t}^{(i)}=\bar{P}+\theta^{(i)}, \forall i \in A, \forall t \geq 0$.
} 
são de domínio público e dizem respeito a mudanças nos fund̦amentos econômicos do ativo. Essas notícias, em geral, rebaixam ou elevam a maioria das avaliações individuais sobre o valor fundamental do ativo. Ao estudarmos o impacto que uma variação positiva (negativa) de $\bar{P}$ exerce sobre a dinâmica de preço do ativo em nosso modelo, procuraremos entender o impacto de notícias positivas (negativas) sobre a dinâmica do preço de um ativo na vida real.

\subsubsection{Fundamentalistas e especuladores}

O conjunto $A$ de todos os agentes consiste de dois subconjuntos: um subconjunto de especuladores $S$ e outro de fundamentalistas $F(A=S \cup F)$, atuando em uma proporção $\alpha \in[0,1]$ no mercado, i.e.,

$$
N_{S} / N=\alpha, \quad N_{F} / N=(1-\alpha)
$$

onde $N_{S}$ e $N_{F}$ denotam o número de especuladores e fundamentalistas, respectivamente $\left(N=N_{S}+N_{F}\right)$. Introduzimos

$$
\bar{S}_{t}:=\left\{\begin{array}{lll}
\sum_{s \in S} X_{t}^{(s)} / N_{S} & \text { se } & S \neq \emptyset \\
0 & \text { se } & S=\emptyset
\end{array} \quad \bar{F}_{t}:=\left\{\begin{array}{lll}
\sum_{s \in F} X_{t}^{(f)} / N_{F} & \text { se } & F \neq \emptyset \\
0 & \text { se } & F=\emptyset
\end{array}\right.\right.
$$

Chamaremos $\bar{S}_{t}$ e $\bar{F}_{t}$ de excessos de demanda relativos ou de médias das atitudes de especuladores e fundamentalistas no tempo $t$, respectivamente. Observamos que o excesso de demanda relativo $\bar{A}_{t}$ é dado pela seguinte média ponderada dos excessos de demanda relativos de especuladores e fundamentalistas:

$$
\bar{A}_{t}=\alpha \bar{S}_{t}+(1-\alpha) \bar{F}_{t}
$$

A identidade (2.8) será usada na Seção 2.2.

\subsubsection{Regra de decisão fundamentalista}

Para um fundamentalista $f \in F$ postulamos que

$$
X_{t}^{(f)}=\left\{\begin{array}{lll}
+1 & \text { se } & P_{t}<P_{t}^{(f)} \\
-1 & \text { caso contrário }
\end{array} \quad \forall f \in F, \quad \forall t=0,1,2, \ldots\right.
$$

Com a regra acima, modelamos que um fundamentalista $f \in F$ baseia sua atitude de investimento somente no preço atual do ativo e em sua avaliação individual sobre o valor 
fundamental do ativo. Ele compra (vende) uma quantidade fixa $q$ do ativo somente se o ativo estiver subvalorizado, i.e, $P_{t}<P_{t}^{(f)}$ (sobrevalorizado $P_{t} \geq P_{t}^{(f)}$ ) de seu ponto de vista individual.

\subsubsection{Regra de decisão especulativa}

A regra de decisão de um especulador $s \in S$ modela o comportamento de um agente de mercado que busca lucro de compra e venda no curto prazo. Denotando por $\Delta P_{t+1}^{(s)}$ a estimativa de retorno formada pelo especulador $s$ no instante $t$ sobre o verdadeiro retorno no instante seguinte dado por $\Delta P_{t+1}:=P_{t+1}-P_{t}$ (ainda não realizado), postulamos

$$
X_{t}^{(s)}=+1(-1) \quad \text { se, e somente se } \Delta P_{t+1}^{(s)}>0(\leq 0)
$$

Ou seja, o agente $s$ compra (vende) uma quantidade fixa do ativo no instante $t$, quando estima que o preço do ativo no instante $t+1$ será maior (menor ou igual) que o preço do ativo no instante $t$. Agora, para a estimativa de retorno $\Delta P_{t+1}^{(s)}$ postularemos inicialmente

$$
\Delta P_{t+1}^{(s)}=J_{1} \Delta P_{t}+J_{2} \bar{S}_{t-1}-J_{3}\left(P_{t}-P_{t}^{(s)}\right) \quad \text { com } \quad J_{1}, J_{2} \geq 0 \quad \text { e } \quad J_{3}>0
$$

onde $\Delta P_{t}:=P_{t}-P_{t-1}$ denota o último retorno observado, $P_{t}^{(s)}$ denota a avaliação sobre o valor fundamental do ativo formada pelo especulador $s$ no instante $t$, relembrando que $\bar{S}_{t-1}=\sum_{s \in S} X_{t-1}^{(s)} / N_{S}$, i.e., que $\bar{S}_{t-1}$ denota a média aritmética das decisões tomadas pelos especuladores no instante anterior.

A seguir, comentaremos os fatores que influenciam esta estimativa e postularemos a regra de decisão dos especuladores. Este fatores são: 1) influência da tendência do preço $\left(J_{1}>0\right)$, onde cada especulador é tanto mais influenciado a estimar que o próximo retorno do ativo seja positivo quanto maior tenha sido o último retorno observado; 2) influência da maioria dos especuladores - contágio de opinião $\left(J_{2}>0\right)$, onde cada especulador $s$ é tanto mais influenciado a orientar suas estimativas ao sinal da maioria das estimativas formadas no instante anterior quanto mais representativa tenha sido esta maioria; e 3) influência da sobrevalorização do ativo $\left(J_{3}>0\right)$, onde cada especulador $s$ é tanto mais influenciado a estimar que o próximo retorno do ativo seja negativo quanto maior for a sobrevalorização do ativo avaliado pelo especulador. De (2.3), (2.10) e (2.11) segue agora

$X_{t}^{(s)}=\left\{\begin{array}{ll}+1 & \text { se } \\ -1 & \text { caso contrário }\end{array} \bar{A}_{t-1}+K \bar{S}_{t-1}-\left(P_{t}-P_{t}^{(s)}\right)>0 \quad \forall s \in S, \forall t=1,2, .(2.1\right.$ 
onde $J:=J_{1} \lambda / J_{3} \geq 0$ e $K:=J_{2} / J_{3} \geq 0$. A regra acima pode ser interpretada da seguinte forma: cada especulador $s$ deixa de seguir a tendência positiva (negativa) do preço e/ou opinião da maioria dos outros especuladores quando ele avaliar que o preço do ativo está demasiadamente acima (abaixo) de seu valor fundamental. A justificativa para esta componente fundamentalista na regra de decisão especulativa (2.12) decorre da tentativa de os especuladores estimarem o impacto que a ação de fundamentalistas excercem sobre incremento de preço resultante no curto prazo. Se, por exemplo, o especulador $s$ estimar que o ativo está demasiadamente sobrevalorizado no instante $t\left(P_{t}-P_{t}^{(s)}\right.$ grande), avaliará também que a maioria dos fundamentalistas estimarão que o preço do ativo está acima de seu valor fundamental, i.e., o especulador $s$ estimará um excesso de demanda fundamentalista negativo no instante $t$. Formalmente, estamos assumindo, portanto, que cada especulador $s \in S$ conhece a regra de decisão fundamentalista (2.9) e sabe que, em média, sua avaliação individual sobre o valor fundamental do ativo é tão boa ou ruim quanto a de qualquer outro fundamentalista, porém, no entanto, desconhece as avaliações individuais $P_{t}^{(f)}, f \in F$, bem como a distribuição $\Phi$ que rege todas as avaliações individuais $P_{t}^{(i)}$, $i \in S \cup F$. Como o incremento de preço tem o mesmo sinal que o excesso de demanda total $\left(P_{t+1}-P_{t}=\lambda \bar{A}_{t}, \lambda>0\right)$, que por sua vez é formado pelo excesso de demanda de especuladores e fundamentalistas $\left(\bar{A}_{t}=\alpha \bar{S}_{t}+(1-\alpha) \bar{F}_{t}\right)$, é natural supor que o especulador $s$ leve em conta $P_{t}-P_{t}^{(s)}$ como uma forma de estimar a contribuição negativa dos fundamentalistas $\left(\lambda(1-\alpha) \bar{F}_{t}\right)$ para o incremento resultante do preço do ativo. Soma-se a esse comportamento o fato de cada especulador partir do mesmo tipo de comportamento por parte de outros especuladores, tanto mais se justifica a componente fundamentalista na regra (2.12). Posteriormente, estudaremos o modelo sob uma outra regra de decisão especulativa.

\subsection{Processo da sobrevalorização do ativo e excesso de demanda relativo}

Na seção anterior construímos um sistema que possui componentes estocásticas e se desenvolve no tempo discreto. Nosso próximo objetivo é estudar as propriedades do processo de preço que se forma neste sistema. Lembramos que o preço no modelo foi designado por $P_{t}$, portanto, o objetivo do estudo é o processo estocástico $\left(P_{t}\right)_{t \geq 0}$. Este objetivo 
está sendo considerado como o principal, pois o preço é uma das quantidades facilmente observadas no mercado real e, portanto, a comparação do comportamento do "preço real" com o comportamento do preço do modelo pode servir como critério de adequação do modelo à realidade.

Estudaremos inicialmente o processo tríplice $\left(P_{t}-\bar{P}, \bar{S}_{t}, \bar{F}_{t}\right)_{t \geq 0}$ que surge no sistema construído. A razão pela qual estudaremos inicialmente o processo tríplice é que, de um lado, o processo $\left(P_{t}\right)_{t \geq 0}$ pode ser facilmente extraído do processo tríplice e, de outro lado, este processo tríplice corresponde à mais reduzida observação do sistema que possui a propriedade Markoviana. Esta propriedade facilita significantemente o estudo do processo.

Vamos, agora, introduzir as notações que serão usadas neste estudo. Designamos com

$$
H_{t}:=P_{t}-\bar{P}
$$

a diferença entre o preço e a avaliação média sobre o valor fundamental do ativo definido em (2.4). Chamaremos $H_{t}$ de sobrevalorização do ativo. Note que, dado $P_{t}, H_{t}$ é a diferença média entre o preço e a avaliação individual sobre o valor fundamental do ativo, i.e., valor esperado de $P_{t}-P_{t}^{(i)}$ (lembramos que $P_{t}^{(i)}, i \in A$, são variáveis aleatórias identicamente distribuídas com valor esperado igual a $\bar{P}$ ). A sobrevalorização do ativo tem a seguinte interpretação: $H_{t}$ corresponde à percepção média dos agentes de quanto o ativo está sobrevalorizado no tempo $t$.

O processo tríplice que estudaremos será denotado por $\left(W_{t}\right)_{t \geq 0}$, onde $W_{t}=\left(H_{t}, \bar{S}_{t}, \bar{F}_{t}\right)$ denota o vetor composto pela sobrevalorização do ativo, pelo excesso de demanda relativo dos especuladores e pelo excesso de demanda relativo dos fundamentalistas. Introduziremos agora as notações para restringir o conjunto ao qual $W_{t}$ pertence. Para um dado número natural $n$, definimos $\Lambda(n) \subset[-1,1]$, tal que

$$
\Lambda(n):= \begin{cases}\{[2(k / n)-1]: k=0,1,2, \ldots, n\} & \text { se } n \neq 0 \\ \{0\} & \text { se } n=0\end{cases}
$$

Para uma quantidade total de agentes $N \in \mathbb{N}$ e uma proporção de especuladores $\alpha$, com $\alpha N \in \mathbb{N}$, definimos

$$
\Omega(\alpha, N):=\mathbb{R} \times \Lambda(\alpha N) \times \Lambda((1-\alpha) N)
$$

Segue agora, de acordo com (2.7) e (2.14), que $W_{t} \in \Omega(\alpha, N)$ para $t=0,1,2, \ldots$ Notamos que para uma dada fração $\alpha \in[0,1]$ e dois números naturais $N$ e $k$ segue $\Omega(\alpha, N) \subset$ 
$\Omega(\alpha, k N)$. Esta propriedade será empregada na Seção 2.3 quando passarmos a quantidade de agentes $N$ para o infinito.

Pela identidade (2.8) e pelas regras de decisão (2.9) e (2.12), as variáveis aleatórias $X_{t+1}^{(s)}, s \in S$, são variáveis aleatórias independentes e identicamente distribuídas dado o vetor $W_{t}(t=0,1,2, \ldots)$. O mesmo vale para as variáveis aleatórias $X_{t+1}^{(f)}, f \in F$ $(t=0,1,2, \ldots)$. Observamos que esta é a razão pela qual o processo tríplice $\left(W_{t}\right)_{t \geq 0}$ é Markoviano.

Para $w \in \Omega(\alpha, N) \subset \mathbb{R} \times[-1,1]^{2}$, denotamos com $Q_{S}(w):=P\left(X_{t+1}^{(s)}=+1 \mid W_{t}=w\right)$ e $Q_{F}(w):=P\left(X_{t+1}^{(f)}=+1 \mid W_{t}=w\right)$ as probabilidades condicionais de $X_{t+1}^{(s)}=1$ e $X_{t+1}^{(f)}=$ 1 dado $W_{t}=w$, respectivamente. Chamaremos $Q_{S}(w)$ e $Q_{F}(w)$ de probabilidades de compra de especuladores e fundamentalistas, respectivamente. De acordo com (2.3), (2.4), (2.8), (2.9), (2.12) e (2.13) é fácil ver que estas probabilidades de compra de especuladores e fundamentalistas são fụnções de $w$, i.e., com domínio em $\Omega(\alpha, N)$, passíveis de extensão ao domínio $\mathbb{R} \times[-1,1]^{2}(\supset \Omega(\alpha, N))$. Estas funções são unicamente determinadas pelo fator $\lambda$ apresentado em (2.3), pela função de distribuição de probabilidade $\Phi$ de (2.5), pela fração de especuladores $\alpha$ em (2.8) e pelas constantes $J$ e $K$ da regra (2.12). Isto é, $Q_{S}: \mathbb{R} \times[-1,1]^{2} \rightarrow[0,1]$ e $Q_{F}: \mathbb{R} \times[-1,1]^{2} \rightarrow[0,1]$, onde

$$
Q_{S}(h, \bar{S}, \bar{F})=\Phi(J \bar{A}+K \bar{S}-(h+\lambda \bar{A})) \quad \text { e } \quad Q_{F}(h, \bar{S}, \bar{F})=\Phi(-(h+\lambda \bar{A}))
$$

onde $\bar{A}:=\alpha \bar{S}+(1-\alpha) \bar{F}$.

Destacamos mais uma vez que o processo $\left(W_{t}\right)_{t \geq 0}$ é Markoviano e que, dado $W_{t-1}=$ $\left(H_{t-1}, \bar{S}_{t-1}, \bar{F}_{t-1}\right)$, o vetor $W_{t}$ é gerado da seguinte forma:

$$
W_{t}=\left(H_{t}, \bar{S}_{t}, \bar{F}_{t}\right) \text { sendo que }\left\{\begin{aligned}
H_{t} & =H_{t-1}+\lambda\left(\alpha \bar{S}_{t-1}+(1-\alpha) \bar{F}_{t-1}\right) \\
\bar{S}_{t} & =\sum_{s \in S} X_{t}^{(s)} / N_{S} \\
\bar{F}_{t} & =\sum_{f \in F} X_{t}^{(f)} / N_{F}
\end{aligned}\right.
$$

onde $X_{t}^{(s)}, s \in S$, e $X_{t}^{(f)}, f \in F$, são realizações independentes de variáveis aleatórias geradas mediante a lei

$$
\begin{aligned}
& X_{t}^{(s)}=\left\{\begin{array}{ll}
+1, & \text { com probabilidade } Q_{S}\left(W_{t-1}\right) \\
-1, & \text { com probabilidade } 1-Q_{S}\left(W_{t-1}\right)
\end{array} \quad \forall s \in S\right. \\
& X_{t}^{(f)}=\left\{\begin{array}{lll}
+1, & \text { com probabilidade } Q_{F}\left(W_{t-1}\right) \\
-1, & \text { com probabilidade } 1-Q_{F}\left(W_{t-1}\right)
\end{array} \quad \forall f \in F\right.
\end{aligned}
$$




\subsection{Limite do processo para grandes populações de agentes (Proposição 1)}

A seguir, deduziremos o sistema dinâmico para o qual a cadeia de Markov $\left(H_{t}, \bar{S}_{t}, \bar{F}_{t}\right)_{t \geq 0}$ converge em probabilidade, quando $N \rightarrow \infty$ para uma fração fixa de especuladores $\alpha$, i.e, $N_{S} / N=\alpha$. Deduziremos também uma estimativa para a velocidade de convergência do processo estocástico para o sistema dinâmico. Partiremos de funções $Q_{S}$ e $Q_{F}$ genéricas para (2.18) e (2.19). Isto porque a Proposição 1 que apresentaremos abaixo será aplicada também a uma outra regra de decisão especulativa, além daquela mencionada em (2.12).

Proposição 1. Sejam $Q_{S}$ e $Q_{F}$ duas funções com domínio em $\mathbb{R} \times[-1,1]^{2}$ e imagem em $[0,1]$ e seja $\alpha \in[0,1]$ um número racional, i.e., $\alpha=p / q$ com $p, q \in \mathbb{N}$ e $p \leq q$. Para um vetor inicial arbitrário $w_{0}:=\left(H_{0}^{(\infty)}, \bar{S}_{0}^{(\infty)}, \bar{F}_{0}^{(\infty)}\right) \in \Omega(\alpha, q)$ definimos a seqüência determinística $w_{t} t=1,2 \ldots$ pela recursão

$$
w_{t}=\left(H_{t}^{(\infty)}, \bar{S}_{t}^{(\infty)}, \bar{F}_{t}^{(\infty)}\right) \quad \text { onde }\left\{\begin{aligned}
H_{t}^{(\infty)} & =H_{t-1}^{(\infty)}+\lambda\left(\alpha \bar{S}_{t-1}^{(\infty)}+(1-\alpha) \bar{F}_{t-1}^{(\infty)}\right) \\
\bar{S}_{t}^{(\infty)} & =E_{S}\left(w_{t-1}\right) \\
\bar{F}_{t}^{(\infty)} & =E_{F}\left(w_{t-1}\right)
\end{aligned}\right.
$$

onde

$E_{S}\left(w_{t-1}\right):=\left\{\begin{array}{ll}2 Q_{S}\left(w_{t-1}\right)-1 & \text { se } \alpha>0 \\ 0 & \text { se } \alpha=0\end{array} ; \quad E_{F}\left(w_{t-1}\right):= \begin{cases}2 Q_{F}\left(w_{t-1}\right)-1 & \text { se }(1-\alpha)>0 \\ 0 & \text { se }(1-\alpha)=0\end{cases}\right.$

Seja $G: \mathbb{R} \times[-1,1]^{2} \rightarrow[-1,1]^{2}$, a função dada por

$$
G(w):=\left(E_{S}(w), E_{F}(w)\right)
$$

Se $G$ for localmente contínua segundo Lipschitz nos pontos $w_{t}, t=0,1,2 \ldots$, i.e., se para todo $w_{t} \in D:=\mathbb{R} \times[-1,1]^{2}$ existirem constantes $\vartheta=\vartheta\left(w_{t}\right)>0$ e $L=L\left(w_{t}\right)>0$, tais que

$$
w \in B_{\vartheta}\left(w_{t}\right) \cap D \Rightarrow\left\|G(w)-G\left(w_{t}\right)\right\|_{\max }<L\left\|w-w_{t}\right\|_{\max }
$$

onde $\|\cdot\|_{\text {max }}$ denota a norma dos máximos (máximo valor absoluto das componentes) e $B_{\vartheta}\left(w_{t}\right)$ uma bola em $\mathbb{R}^{3}$ de raio $\vartheta$, centrada em $w_{t}$, 
então para a cadeia de Markov $\left(W_{t}\right)_{t \geq 0}$ definida por (2.17), (2.18) e (2.19) vale a seguinte convergência em probabilidade: $\forall t \geq 0, \varepsilon>0$ vale

$$
\begin{aligned}
\quad \lim & \mathbb{P}\left(\left\|W_{t+1}-w_{t+1}\right\|_{\max }>\varepsilon \mid W_{0}=w_{0}\right)=0 \\
k & \rightarrow \infty \\
N & =k q \\
N_{S} / N & =\alpha
\end{aligned}
$$

com a seguinte cota superior para a probabilidade em (2.23) que vale para $N \in\{k q$ : $k \in \mathbb{N}\}$ (número total de agentes) suficientemente grande e sob a condição de que $\varepsilon-$ $\Pi_{t+1} / \sqrt[4]{N} \geq 0$

$$
2 t(N+1) \exp \left\{-\frac{1}{2} \underline{\alpha} M_{t} \sqrt{N}\right\}+2(N+1) \exp \left\{-\frac{1}{2} \underline{\alpha} N\left[\varepsilon-\Pi_{t+1} / \sqrt[4]{N}\right]^{2}\right\}
$$

onde $\underline{\alpha}:=\operatorname{Min}\{\alpha, 1-\alpha\}$ se $0<\alpha<1$ e $\underline{\alpha}:=1$ se $\alpha=0$ ou $\alpha=1$. As constantes $\Pi_{t} e$ $M_{t}(t=0,1,2, \ldots)$ são definidas da seguinte forma:

$$
\begin{array}{ll}
\Pi_{0}=1, \quad \Pi_{t+1}=e^{c t} \prod_{\tau=0}^{t} L\left(w_{\tau}\right) & \forall t \geq 0 \\
M_{t}=\left[\left(e^{c}-1\right) \operatorname{Min}_{0 \leq \kappa \leq t}\left(\Pi_{\kappa}\right)\right]^{2} & \forall t \geq 0
\end{array}
$$

onde c é uma constante positiva qualquer e $L\left(w_{t}\right), t=0,1,2, \ldots$, constates de Lipschitz no sentido (2.22).

A Proposição 1 afirma que a cadeia de Markov $\left(W_{t}\right)_{t \geq 0}$ converge para o sistema determinístico $\left(w_{t}\right)_{t \geq 0}$ quando o número de agentes $N$ tende ao $\infty$ sob uma dada fração $\alpha$ constante de especuladores. A idéia intuitiva da demonstração é a seguinte: dado $W_{0}=w_{0}$, segue que $H_{1}$ depende deterministicamente de $w_{0}$. Como, por construção, $H_{1}^{(\infty)}$ depende de $w_{0}$ da mesma forma que $H_{1}$ depende de $w_{0}$, segue $H_{1}=H_{1}^{(\infty)}$. Por outro lado, dado $W_{0}=w_{0}$, as decisões dos especuladores no instante seguinte $X_{1}^{(s)}, s \in S$ são variáveis aleatórias independentes e identicamente distribuídas. O mesmo vale para as variáveis $X_{1}^{(f)}, f \in F$. Segundo a lei forte dos grandes números, as médias empíricas $\bar{S}_{1}$ e $\bar{F}_{1}$ convergem em probabilidade para os respectivos valores esperados de $X_{1}^{(s)}$ e $X_{1}^{(f)}$ dado $W_{0}=w_{0}$. Por construção, estes valores esperados são $\bar{S}_{1}^{(\infty)}$ e $\bar{F}_{1}^{(\infty)}$, respectivamente. Para uma grande quantidade de agentes, podemos esperar que $W_{1}$ está próximo 
de $w_{1}$. Posto isso, podemos obter intuitivamente uma aproximação para $W_{2}$ aplicando novamente a lei forte dos grandes números, dado que $W_{1}=w_{1}$. Intuitivamente obtemos a convergência para $t>1$ através de $t$ aplicações sucessivas da lei forte dos grandes números. A demonstração rigorosa para $t>1$, bem como a obtenção da estimativa da probabilidade de desvio entre $W_{t}$ e $w_{t}$, expressão (2.30), é simples, porém, longa e não trivial. A construção da demonstração é baseada em estimativas para grandes desvios de variáveis aleatórias discretas. Esta prova será apresentada na Seção 2.3.2.

Sistemas dinâmicos limites para grandes populações de agentes. Devido à identidade (2.8) e à Proposição 1 , o excesso de demanda $\bar{A}_{t}$ converge em probabilidade para

$$
\bar{A}_{t}^{(\infty)}:=\alpha \bar{S}_{t}^{(\infty)}+(1-\alpha) \bar{F}_{t}^{(\infty)}
$$

quando a quantidade de agentes $N$ tende ao infinito sob $N_{S} / N=\alpha$. Chamaremos $P_{t}^{(\infty)}$, $H_{t}^{(\infty)}, \bar{S}_{t}^{(\infty)}, \bar{F}_{t}^{(\infty)}, \bar{A}_{t}^{(\infty)}$ e $w_{t}$ com os mesmos termos usados para $P_{t}, H_{t}, \bar{S}_{t}, \bar{F}_{t}, \bar{A}_{t}$ e $W_{t}$, respectivamente. Ou seja, chamaremos $P_{t}^{(\infty)}$ simplesmente de "preço do ativo", $H_{t}^{(\infty)}$ de "sobrevalorização do ativo" e assim por diante. Quando houver dúvida, chamaremos $P_{t}^{(\infty)}$ de "limite para grandes populações do preço do ativo", $H_{t}^{(\infty)}$ de "limite para grandes populações da sobrevalorização do ativo" e assim por diante. Para não carregar a notação e sempre que não houver dúvida, omitiremos também " $(\infty)$ " nas notações $P_{t}^{(\infty)}, H_{t}^{(\infty)}$, $\bar{S}_{t}^{(\infty)}, \bar{F}_{t}^{(\infty)}$ e $\bar{A}_{t}^{(\infty)}$ substituindo estas notações por $P_{t}, H_{t}, \bar{S}_{t}, \bar{F}_{t}$ e $\bar{A}_{t}$, respectivamente.

Apresentamos a seguir uma vizualização esquemática do processo estocástico $\left(W_{t}\right)_{t \geq 0}$, bem como de sua convergência para o sistema dinâmico $\left(w_{t}\right)_{t \geq 0}$. 


\section{PROCESSO ESTOCÁSTICO}

$$
\begin{gathered}
{\left[\begin{array}{c}
H_{0}^{(\infty)} \\
\bar{S}_{0}^{(\infty)} \\
\bar{F}_{0}^{(\infty)}
\end{array}\right] \stackrel{Q_{s,} Q_{F},}{\longrightarrow}\left[\begin{array}{c}
\left(X_{1}^{(s)}, s \in S\right) \\
\left(X_{1}^{(f)}, f \in F\right) \\
\Downarrow \\
\left(H_{1}, \bar{S}_{1}, \bar{F}_{1}\right)
\end{array}\right] \stackrel{Q_{s}, Q_{F}}{\longrightarrow}\left[\begin{array}{c}
\left(X_{2}^{(s)}, s \in S\right) \\
\left(X_{2}^{(f)}, f \in F\right) \\
\Downarrow \\
\left(H_{2}, \bar{S}_{2}, \bar{F}_{2}\right)
\end{array}\right]} \\
\Rightarrow\left(H_{t}, \bar{S}_{t}, \bar{F}_{t}\right)_{t \geq 0} \quad \text { é um processo estocástico Markoviano } \\
\text { DO ESTOCÁSTICO PARA O DETERMINÍSTICO }
\end{gathered}
$$

\section{DO ESTOCÁSTICO PARA O DETERMINÍSTICO}

Mantendo a fração $\alpha\left(=N_{S} / N\right)$ de especuladores constante, segue abaixo a convergência de $W_{1}$ para $w_{1}$ quando a quantidade de agentes $N$ tende ao infinito.

$$
\begin{array}{ll}
H_{1}=H_{0}+\lambda \bar{A}_{0}=H_{0}^{(\infty)}+\lambda \bar{A}_{0}^{(\infty)} & =: H_{1}^{(\infty)} \\
\bar{S}_{1}=\frac{\sum_{s \in S} X_{1}^{(s)}}{N_{S}} \rightarrow \mathbb{E}\left(X^{(s)} \mid W_{0}=w_{0}\right)=2 Q_{S}\left(w_{0}\right)-1=: \bar{S}_{1}^{(\infty)} \\
\bar{F}_{1}=\frac{\sum_{f \in F} X_{1}^{(f)}}{N_{F}} \rightarrow \mathbb{E}\left(X^{(f)} \mid W_{0}=w_{0}\right)=2 Q_{S}\left(w_{0}\right)-1=: \bar{F}_{1}^{(\infty)}
\end{array}
$$

onde $\bar{A}_{0}^{(\infty)}:=\alpha \bar{S}_{0}^{(\infty)}+(1-\alpha) \bar{F}_{0}^{(\infty)}$.

A convergência de $W_{2}$ para $w_{2}$ dado $W_{1}=w_{1}$ e $N_{S} / N=\alpha$ (constante) segue da seguinte forma:

$$
\begin{aligned}
& \frac{N_{S}}{N} \frac{\sum_{s \in S} X_{1}^{(s)}}{N_{S}}+\frac{N_{F}}{N} \frac{\sum_{f \in F} X_{1}^{(f)}}{N_{F}}=\bar{A}_{1} \\
& \begin{array}{lll}
N \rightarrow \infty & N_{S}=\alpha
\end{array} \quad \downarrow \quad \downarrow \quad \downarrow \\
& \alpha\left[2 Q_{S}\left(W_{0}\right)-1\right]+(1-\alpha)\left[2 Q_{F}\left(W_{0}\right)-1\right]=: \bar{A}_{1}^{(\infty)} \\
& H_{2}=H_{1}^{(\infty)}+\lambda \bar{A}_{1} \rightarrow H_{1}^{(\infty)}+\lambda \bar{A}_{1}^{(\infty)} \quad=: H_{2}^{(\infty)} \\
& \bar{S}_{2}=\frac{\sum_{s \in S} X_{2}^{(s)}}{N_{S}} \rightarrow \mathbb{E}\left(X^{(s)} \mid W_{1}=w_{1}\right)=2 Q_{S}\left(w_{1}\right)-1=: \bar{S}_{2}^{(\infty)} \\
& \bar{F}_{2}=\frac{\sum_{f \in F} X_{2}^{(f)}}{N_{F}} \rightarrow \mathbb{E}\left(X^{(f)} \mid W_{1}=w_{1}\right)=2 Q_{S}\left(w_{1}\right)-1=: \bar{F}_{2}^{(\infty)}
\end{aligned}
$$




\subsubsection{Corolários da Proposição 1}

Nesta seção apresentaremos uma estimativa alternativa à (2.24) para a probabilidade de desvio entre $W_{t}$ e $w_{t}$. Esta nova estimativa é dada por (2.30). A estimativa (2.30) permite um maior controle sobre a probabilidade de desvio entre $W_{t}$ e $w_{t}$. De acordo com a nova estimativa (2.30), concluímos que quanto mais rápido a seqüencia $\left(L\left(w_{t}\right)\right)_{t \geq 0}$ convergir para zero, menor (melhor) será a estimativa da probabilidade de desvio entre o processo estocástico $W_{t}$ e o sistema dinâmico $w_{t}$. Esta implicação será esclarecida logo após a prova do Corolário 1.

Corolário 1. Se existirem constantes de Lipschitz $\left(L\left(w_{t}\right)\right)_{t \geq 0}$, tais que o seu produtório convirja para 0 exponencialmente rápido, i.e., se existirem constantes $b \geq 1 c>0$, tais que $\prod_{\tau=0}^{t} L\left(w_{\tau}\right) \leq b e^{-c t} \quad \forall t \geq 0$, então podemos estimar a probabilidade em (2.23) por

$$
2 t(N+1) \exp \left\{-\frac{1}{2} \underline{\alpha}\left(e^{c}-1\right)^{2} \sqrt{N}\right\}+2(N+1) \exp \left\{-\frac{1}{2} \underline{\alpha} N[\varepsilon-b / \sqrt[4]{N}]^{2}\right\}
$$

Demonstração do Corolário 1. Como qualquer constante maior que uma constante de Lipschitz também é uma constante de Lipschitz, podemos admitir, sem perda de generalidade,

$$
\prod_{\tau=0}^{t} L\left(w_{\tau}\right)=b e^{-c t} \quad \forall t \geq 0
$$

substituindo (2.31) em (2.25) segue o resultado.

Observação. O Corolário 1 relaciona a taxa de decaimento exponencial do produtório das constantes de Lipschitz $\left(L\left(w_{t}\right)\right)_{t \geq 0}$ com a velocidade de convergência da probabilidade em (2.23). Note que quanto maior for $c>0$ e menor for $b \geq 1$ tanto menor será (2.30). Portanto, quanto mais rápido $\left(L\left(w_{t}\right)\right)_{t \geq 0}$ convergir para zero, menor será a estimativa da probabilidade de desvio entre $W_{t}$ e $w_{t}$.

Corolário 2. Seja $G: \mathbb{R} \times[-1,1]^{2} \rightarrow[0,1]^{2}$ em (2.21) diferenciável. Denotamos com $G_{i, j}(i=1,2$ e $j=1,2,3)$ a derivada parcial da i-ésima componente de $G$ pela $j$-ésima componente do argumento de $G$. Denotamos com $G^{\prime}$ a função dada por $G^{\prime}: \mathbb{R} \times[-1,1] \rightarrow$ $[0,1]^{2}$, onde $G^{\prime}=\left(\sum_{j=1}^{3} G_{1, j}, \sum_{j=1}^{3} G_{2, j}\right)$. 
Se $w^{*} \in \mathbb{R} \times[-1,1]^{2}$ for o ponto onde $G^{\prime}$ tem a maior norma e valer $\left\|G^{\prime}\left(w^{*}\right)\right\|_{\max }<1$, i.e., $\left\|G^{\prime}\left(w^{*}\right)\right\|_{\max }=\operatorname{Max}\left\{\left\|G^{\prime}(w)\right\|_{\text {max }}: w \in \mathbb{R} \times[-1,1]^{2}\right\}<1$, então vale a estimativa do Corolário 1 para

$$
c=-\log \left\|G^{\prime}\left(w^{*}\right)\right\|_{\text {max }} \quad e \quad b=1
$$

Demonstração do Corolário 2. Como $\left\|G^{\prime}\left(w^{*}\right)\right\|_{\max }$ é o valor máximo que a norma de $G^{\prime}$ pode assumir, $\left\|G^{\prime}\left(w^{*}\right)\right\|_{\max }$ é uma constante de Lipschitz universal. Portanto,

$$
L\left(w_{t}\right):=\left\|G^{\prime}\left(w^{*}\right)\right\|_{\max }
$$

é uma constante de Lipschitz local em $w_{t}(t=0,1,2 \ldots)$. Como $\left\|G^{\prime}\left(w^{*}\right)\right\|_{\max }<1$ temos $c>0$ em conformidade com (2.32). De (2.32) e (2.33) está claro que $\prod_{\tau=0}^{t} L\left(w_{\tau}\right)=$ $b e^{-c t} \quad \forall t \geq 0$. O resultado segue o do Corolário 1 .

O resultado do Corolário 2 relaciona a estimativa da probabilidade de desvio entre $W_{t}$ e $w_{t}$ dada em (2.24) com um "grau de estabilidade" do sistema dinâmico (determinístico) de suas médias $\left(w_{t}\right)_{t \geq 0}$ dado em (2.20). Para entender isso, note inicialmente que, por construção, $w_{t}=\left(\mathbb{E}\left(H_{t} \mid W_{0}=w_{0}\right), \mathbb{E}\left(\bar{S}_{t} \mid W_{0}=w_{0}\right), \mathbb{E}\left(\bar{F}_{t} \mid W_{0}=w_{0}\right)\right)$. Suponha, agora, que $w^{*}$ seja o único equilíbrio globalmente estável do sistema dinâmico $\left(w_{t}\right)_{t \geq 0}$, i.e., para qualquer vetor inicial $w_{0} \in \mathbb{R} \times[-1,1]^{2}, \lim _{t \rightarrow \infty} w_{t}=w^{*}$. Como o sistema dinâmico das médias é dado por

$$
w_{t}=\left(H_{t-1}^{(\infty)}+\lambda\left[\alpha G_{1}\left(w_{t-1}\right)+(1-\alpha) G_{1}\left(w_{t-1}\right)\right] ; G_{1}\left(w_{t-1}\right) ; G_{2}\left(w_{t-1}\right)\right)
$$

é fácil ver que quanto menor for $\left\|G^{\prime}\left(w^{*}\right)\right\|_{\text {max }}$, maior será a velocidade de convergência do sistema dinâmico $\left(w_{t}\right)_{t \geq 0}$ para o equilíbrio $w^{*}$ nas proximidades deste equilíbrio. Neste sentido, - $\log \left\|G^{\prime}\left(w^{*}\right)\right\|_{\text {max }}$ expressa um "grau de estabilidade" do equilíbrio global $w^{*}$. Por outro lado, segundo o Corolário 2, a constante $c$ pode ser escolhida tão grande quanto $-\log \left\|G^{\prime}\left(w^{*}\right)\right\|_{\max }$. Ou seja, quanto maior for $-\log \left\|G^{\prime}\left(w^{*}\right)\right\|_{\max }$, i.e., quanto mais estável for o equilíbrio $w^{*}$, menor será a estimativa (2.30) do Corolário 1.

Veremos que, sob algumas suposições, a cadeia de Markov $\left(W_{t}\right)_{t \geq 0}$ dada em por (2.17), (2.18) e (2.19) satisfaz as condições do Corolário 2. 
Uma vez que $w^{*}$ é o único equilíbrio globalmente estável do sistema dinâmico $\left(w_{t}\right)_{t \geq 0}$, temos que $w_{t}$ está próximo de $w^{*}$ para $t$ suficientemente grande. Supondo continuidade de $G^{\prime}$, isto implica que $\left\|G^{\prime}\left(w_{t}\right)\right\|_{\max }$ está próximo de $\left\|G^{\prime}\left(w^{*}\right)\right\|_{\max }$ para $t$ suficientemente grande. Portanto, neste caso, as constantes de Lipschitz em (2.33) são, a partir de um certo instante $t$, essencialmente as menores (melhores) possíveis e, portanto, a maior constante de decaimento para o produtório de constantes de Lipschitz é de fato - log $\left\|G^{\prime}\left(w^{*}\right)\right\|_{\text {max }}$. Neste contexto, é interessante observar que, quando $\left\|G^{\prime}\left(w^{*}\right)\right\|=1$, temos $c=0$ e a estimativa (2.30) para a probabilidade em (2.23) se perde, i.e., assume um valor maior que 1. Uma estimativa inferior para a probabilidade em (2.23) nos esclareceria se $\left\|G^{\prime}\left(w^{*}\right)\right\|=1$ é crítico ou não para a probabilidade de desvio entre $W_{t}$ e $w_{t}$, quando $w^{*}$ é o único equilíbrio globalmente estável do sistema dinâmico $\left(w_{t}\right)_{t \geq 0}$ (sistema dinâmico dos valores esperados de $W_{t}$ ). Este tema não será abordado neste trabalho.

Observamos ainda que a condição do Corolário 1 pode valer mesmo quando a seqüência $\left(L\left(w_{t}\right)\right)_{t \geq 0}$ assume, um número infinito vezes, valores maiores que um. Para tanto, basta que $\prod_{t}^{\infty} L\left(w_{t}\right)$ convirja exponencialmente rápido para zero. Este é o caso de alguns ciclos predominantemente determinísticos do processo $\left(W_{t}\right)_{t>0}$ em que a norma das derivadas nas médias $\left\|G^{\prime}\left(w_{t}\right)\right\|_{\text {max }}$ assume valores menores que 1 em maior freqüência do que valores maiores que 1. Veremos essa situação quando o sistema dinâmico das médias $\left(w_{t}\right)_{t \geq 0}$ da cadeia de Markov dada em (2.17), (2.18) e (2.19) entra em certos ciclos, que, no contexto do modelo apresentado, corresponderam à ocorrência de sucessivas bolhas especulativas e crashes de preço do ativo em um sentido ainda a ser explicado. 


\subsubsection{Demonstração da Proposição 1}

Vamos introduzir inicialmente as seguintes notações: sejam

$$
\begin{aligned}
B_{\varepsilon}\left(w_{t+1}\right) & :=\left\{w \in \Omega(\alpha, N):\left\|w-w_{t+1}\right\|_{\max } \leq \varepsilon\right\} \\
B_{\varepsilon}\left(H_{t+1}^{(\infty)}\right) & :=\left\{h \in \mathbb{R}:\left|h-H_{t+1}^{(\infty)}\right| \leq \varepsilon\right\} \\
B_{\varepsilon}\left(\bar{S}_{t+1}^{(\infty)}\right) & :=\left\{\bar{S} \in \Lambda(\alpha N):\left|\bar{S}-\bar{S}_{t+1}^{(\infty)}\right| \leq \varepsilon\right\} \\
B_{\varepsilon}\left(\bar{F}_{t+1}^{(\infty)}\right) & :=\left\{\bar{F} \in \Lambda((1-\alpha) N):\left|\bar{F}-\bar{F}_{t+1}^{(\infty)}\right| \leq \varepsilon\right\}
\end{aligned}
$$

as notações para as vizinhanças de $w_{t+1}, H_{t+1}^{(\infty)}, \bar{S}_{t+1}^{(\infty)}$ e $\bar{F}_{t+1}^{(\infty)}$, respectivamente. Denotamos com $\bar{B}_{\varepsilon}\left(w_{t+1}\right):=\Omega(\alpha, N) / B_{\varepsilon}\left(w_{t+1}\right), \bar{B}_{\varepsilon}\left(H_{t+1}^{(\infty)}\right):=\mathbb{R} / B_{\varepsilon}\left(H_{t+1}^{(\infty)}\right), \bar{B}_{\varepsilon}\left(\bar{S}_{t+1}^{(\infty)}\right):=$ $\Lambda(\alpha N) / B_{\varepsilon}\left(\bar{S}_{t+1}^{(\infty)}\right)$ e $\bar{B}_{\varepsilon}\left(\bar{F}_{t+1}^{(\infty)}\right):=\Lambda((1-\alpha) N) / B_{\varepsilon}\left(\bar{F}_{t+1}^{(\infty)}\right)$ os respectivos complementos destas vizinhanças. Para $t, \tau \in \mathbb{N}$ e $w \in \Omega(\alpha, N)$, sejam

$$
\begin{aligned}
\mu_{\tau}\left(B_{\varepsilon}\left(w^{\prime}\right) \mid w\right) & :=\mathbb{P}\left(W_{t+\tau} \in B_{\varepsilon}\left(w^{\prime}\right) \mid W_{t}=w\right) \quad \forall w^{\prime} \in \Omega(\alpha, N) \\
\mu_{\tau}\left(B_{\varepsilon}(h) \mid w\right) & :=\mathbb{P}\left(H_{t+\tau} \in B_{\varepsilon}(h) \mid W_{t}=w\right) \quad \forall h \in \mathbb{R} \\
\mu_{\tau}\left(B_{\varepsilon}(\bar{S}) \mid w\right) & :=\mathbb{P}\left(\bar{S}_{t+\tau} \in B_{\varepsilon}(\bar{S}) \mid W_{t}=w\right) \quad \forall \bar{S} \in \Lambda(\alpha N) \\
\mu_{\tau}\left(B_{\varepsilon}(\bar{F}) \mid w\right) & :=\mathbb{P}\left(\bar{F}_{t+\tau} \in B_{\varepsilon}(\bar{F}) \mid W_{t}=w\right) \quad \forall \bar{F} \in \Lambda((1-\alpha) N) \\
\mu_{\tau}\left(w^{\prime} \mid w\right) & :=\mathbb{P}\left(W_{t+\tau}=w^{\prime} \mid W_{t}=w\right) \quad \forall w^{\prime} \in \Omega(\alpha, N)
\end{aligned}
$$

De maneira análoga, introduzimos as notações $\mu_{\tau}\left(\bar{B}_{\varepsilon}\left(w^{\prime}\right) \mid w\right), \mu_{\tau}\left(\bar{B}_{\varepsilon}(h) \mid w\right), \mu_{\tau}\left(\bar{B}_{\varepsilon}(\bar{S}) \mid w\right)$ e $\mu_{\tau}\left(\bar{B}_{\varepsilon}(\bar{F}) \mid w\right)$ (paras as probabilidades condicionais dos complementos). Note que as definições de (2.35) até (2.39) não dependem de $t$, pois a cadeia de Markov $\left(W_{t}\right)_{t \geq 0}$ é homogênea no tempo, com probabilidades de transição $\mu_{1}\left(w^{\prime} \mid w\right) \forall w^{\prime}, w \in \Omega(\alpha, N)$.

A prova consistirá em mostrar que para todo $\varepsilon>0$ e $t=0,1,2, \ldots$ existe $N_{t}(\varepsilon) \in \mathbb{N}$, tal que

$$
\forall N>N_{t}(\varepsilon): \mu_{t}\left(\bar{B}_{\varepsilon}\left(w_{t+1}\right) \mid w_{0}\right) \leq \text { "cota superior dada em (2.24)" }
$$

A prova será dividida em três partes.

1) Primeiro, mostraremos que para todo $t \geq 0$ vale

$$
\mu_{t+1}\left(\bar{B}_{\varepsilon}\left(w_{t+1}\right) \mid w_{0}\right) \leq \mu_{t}\left(\bar{B}_{\varepsilon_{t}(N)}\left(w_{t}\right) \mid w_{0}\right)+\mu_{1}\left(\bar{B}_{\varepsilon}\left(w_{t+1}\right) \mid w_{t}^{*}\right)
$$


onde

$$
\varepsilon_{t}(N):=\Pi_{t+1} /\left(L\left(w_{t}\right) \sqrt[4]{N}\right)
$$

e onde $w_{t}^{*} \in B_{\varepsilon_{t}(N)}\left(w_{t}\right)$ é definido de tal sorte a maximizar a probabilidade condicional $\mu_{1}\left(\bar{B}_{\varepsilon}\left(w_{t+1}\right) \mid w_{t}^{*}\right)$, isto é,

$$
\mu_{1}\left(\bar{B}_{\varepsilon}\left(w_{t+1}\right) \mid w_{t}^{*}\right)=\operatorname{Max}\left\{\mu_{1}\left(\bar{B}_{\varepsilon}\left(w_{t+1}\right) \mid w\right): w \in B_{\varepsilon_{t}(N)}\left(w_{t}\right)\right\}
$$

2) Em seguida, mostraremos que para todo $\varepsilon>0$ e $t=0,1,2, \ldots$ existe $N_{t}(\varepsilon) \in \mathbb{N}$, tal que, para $N>N_{t}(\varepsilon)$, vale

$$
\mu_{1}\left(\bar{B}_{\varepsilon}\left(w_{t+1}\right) \mid w_{t}^{*}\right) \leq 2(N+1) \exp \left\{-\frac{1}{2} \underline{\alpha} N\left[\varepsilon-\Pi_{t+1} / \sqrt[4]{N}\right]^{2}\right\}
$$

3) Por último, mostraremos que para todo $t=0,1,2, \ldots$, existe $N_{t} \in \mathbb{N}$, tal que, para $N>N_{t}$, vale

$$
\mu_{t}\left(\bar{B}_{\varepsilon_{t}(N)}\left(w_{t}\right) \mid w_{0}\right) \leq 2 t(N+1) \exp \left\{-\frac{1}{2} \underline{\alpha} M_{t} \sqrt{N}\right\}
$$

onde $\underline{\alpha}:=\operatorname{Min}\{\alpha, 1-\alpha\}$ se $0<\alpha<1$ e $\underline{\alpha}:=1$ se $\alpha=0$ ou $\alpha=1$.

De (2.41), (2.44) e (2.45) segue o resultado em (2.40).

Demonstração de (2.41). Para $t \geq 0$ calculamos inicialmente $\mu_{t+1}\left(\bar{B}_{\varepsilon}\left(w_{t+1}\right) \mid w_{0}\right)$

$$
\begin{aligned}
\mu_{t+1}\left(\bar{B}_{\varepsilon}\left(w_{t+1}\right) \mid w_{0}\right) & =\sum_{w \in \Omega(\alpha, N)} \mu_{1}\left(\bar{B}_{\varepsilon}\left(w_{t+1}\right) \mid w\right) \mu_{t}\left(w \mid w_{0}\right) \\
& =\underbrace{\sum_{w \in \bar{B}_{\varepsilon_{t}(N)}\left(w_{t}\right)}}_{I}+\underbrace{\sum_{w \in B_{\varepsilon_{t}(N)}\left(w_{t}\right)}}_{I I}=(\star)
\end{aligned}
$$

Agora, I e II podem ser estimados como segue:

$$
I=\sum_{w \in \bar{B}_{\varepsilon_{t}(N)}\left(w_{t}\right)} \underbrace{\mu_{1}\left(\bar{B}_{\varepsilon}\left(w_{t+1}\right) \mid w\right)}_{\leq 1} \mu_{t}\left(w \mid w_{0}\right) \leq \mu_{t}\left(\bar{B}_{\varepsilon_{t}(N)}\left(w_{t}\right) \mid w_{0}\right)
$$




$$
\begin{aligned}
I I=\sum_{w \in B_{\varepsilon_{t}(N)}\left(w_{t}\right)} \mu_{1}\left(\bar{B}_{\varepsilon}\left(w_{t+1}\right) \mid w\right) \mu_{t}\left(w \mid w_{0}\right) & \leq \sum_{w \in B_{\varepsilon_{t}(N)}\left(w_{t}\right)} \mu_{1}(\bar{B}_{\varepsilon}\left(w_{t+1}\right) \mid \underbrace{w_{t}^{*}}_{(2.43)}) \mu_{t}\left(w \mid w_{0}\right) \\
& =\mu_{1}\left(\bar{B}_{\varepsilon}\left(w_{t+1}\right) \mid w_{t}^{*}\right) \sum_{w \in B_{\varepsilon_{t}(N)}\left(w_{t}\right)} \mu_{t}\left(w \mid w_{0}\right) \\
& =\mu_{1}\left(\bar{B}_{\varepsilon}\left(w_{t+1}\right) \mid w_{t}^{*}\right) \underbrace{\mu_{t}\left(B_{\varepsilon_{t}(N)}\left(w_{t}\right) \mid w_{0}\right)}_{\leq 1} \\
& \leq \mu_{1}\left(\bar{B}_{\varepsilon}\left(w_{t+1}\right) \mid w_{t}^{*}\right)
\end{aligned}
$$

Demonstração de (2.44). A prova (2.44) é direta, i.e., ela não se dará por indução. Para $t \geq 0$, estimaremos abaixo $\mu_{1}\left(\bar{B}_{\varepsilon}\left(w_{t+1}\right) \mid w_{t}^{*}\right)$. Dado que a vizinhança $\bar{B}_{\varepsilon}\left(w_{t+1}\right)$ está definida pela norma $\|\cdot\|_{\max }$ (máximo valor absoluto das componentes), temos inicialmente

$$
\mu_{1}\left(\bar{B}_{\varepsilon}\left(w_{t+1}\right) \mid w_{t}^{*}\right) \leq \mu_{1}\left(\bar{B}_{\varepsilon}\left(H_{t+1}^{(\infty)}\right) \mid w_{t}^{*}\right)+\mu_{1}\left(\bar{B}_{\varepsilon}\left(\bar{S}_{t+1}^{(\infty)}\right) \mid w_{t}^{*}\right)+\mu_{1}\left(\bar{B}_{\varepsilon}\left(\bar{F}_{t+1}^{(\infty)}\right) \mid w_{t}^{*}\right)
$$

Como $H_{t+1}=H_{t}+\lambda\left(\alpha \bar{S}_{t}+(1-\alpha) \bar{F}_{t}\right)$ (depende determinística e continuamente de $\left.W_{t}=\left(H_{t}, \bar{S}_{t}, \bar{F}_{t}\right)\right)$ e $w_{t}^{*} \in B_{\varepsilon_{t}(N)}\left(w_{t}\right)$, com $\left.w_{t}=\left(H_{t}^{(\infty)}, \bar{S}_{t}^{(\infty)}, \bar{F}_{t}^{(\infty)}\right)\right)$, segue que para todo $\varepsilon>0$ existe $N_{t}^{h}(\varepsilon)$, tal que

$$
\forall N>N_{t}^{h}(\varepsilon): \quad \mu_{1}\left(\bar{B}_{\varepsilon}\left(H_{t+1}^{(\infty)}\right) \mid w_{t}^{*}\right)=0
$$

Portanto, devido a (2.46) e (2.47), temos, para $N>N_{t}^{h}(\varepsilon)$,

$$
\mu_{1}\left(\bar{B}_{\varepsilon}\left(w_{t+1}\right) \mid w_{t}^{*}\right) \leq \mu_{1}\left(\bar{B}_{\varepsilon}\left(\bar{S}_{t+1}^{(\infty)}\right) \mid w_{t}^{*}\right)+\mu_{1}\left(\bar{B}_{\varepsilon}\left(\bar{F}_{t+1}^{(\infty)}\right) \mid w_{t}^{*}\right)
$$

A seguir, provaremos que para todo $\varepsilon$ existe $N_{t}^{S}(\varepsilon)>0$, tal que

$\forall N>N_{t}^{S}(\varepsilon): \begin{cases}\mu_{1}\left(\bar{B}_{\varepsilon}\left(\bar{S}_{t+1}^{(\infty)}\right) \mid w_{t}^{*}\right)=0 & \text { se } \quad \alpha=0 \\ \mu_{1}\left(\bar{B}_{\varepsilon}\left(\bar{S}_{t+1}^{(\infty)}\right) \mid w_{t}^{*}\right) \leq(\alpha N+1) \exp \left\{-\frac{1}{2} \alpha N[\varepsilon-\delta]^{2}\right\} & \text { se } \quad \alpha>0\end{cases}$

onde

$$
\delta:=\Pi_{t+1} / \sqrt[4]{N}
$$

Demonstração de (2.49). Para $\alpha=0$, a prova segue imediatamente da definição de $\left(W_{t}\right)_{t \geq 0}$ e $\left(w_{t}\right)_{t \geq 0}$, pois neste caso $\bar{S}_{t}=\bar{S}_{t}^{(\infty)}=0, \forall t \geq 0$. 
Vamos supor agora $\alpha>0$. Observemos inicialmente que, independentemente dos valores assumidos por $E_{S}\left(w_{t}\right), E_{S}\left(w_{t}^{*}\right), \delta$ e $\varepsilon$, sempre vale a seguinte implicação:

$$
\left|E_{S}\left(w_{t}\right)-E_{S}\left(w_{t}^{*}\right)\right| \leq \delta<\varepsilon \Rightarrow \bar{B}_{\varepsilon}\left(E_{S}\left(w_{t}\right)\right) \subset \bar{B}_{\varepsilon-\delta}\left(E_{S}\left(w_{t}^{*}\right)\right)
$$

Vamos supor que exista $N_{t}^{S}(\varepsilon)>0$, tal que o lado esquerdo da implicação (2.51) esteja assegurado sempre que $N>N_{t}^{S}(\varepsilon)$ (isso será provado abaixo). Sob esta suposição e lembrando que $\bar{S}_{t+1}^{(\infty)}=E_{S}\left(w_{t}\right)$, vale para qualquer $N>N_{t}^{S}(\varepsilon)$

$$
\mu_{1}\left(\bar{B}_{\varepsilon}\left(\bar{S}_{t+1}^{(\infty)}\right) \mid w_{t}^{*}\right) \leq \mu_{1}\left(\bar{B}_{\varepsilon-\delta}\left(E_{S}\left(w_{t}^{*}\right)\right) \mid w_{t}^{*}\right)=(\star \star)
$$

Retomemos agora que $\bar{S}_{t+1}$ dado $W_{t}=w_{t}^{*}$ corresponde à média aritmética de $\alpha N$ variáveis aleatórias independentes e identicamente distribuídas assumindo +1 e -1 com probabilidades $p^{*}:=Q_{S}\left(w_{t}^{*}\right)$ e $1-p^{*}$, respectivamente com valor esperado $\bar{S}_{t+1}^{*}:=E_{S}\left(w_{t}^{*}\right)$. Observemos também que para cada $\bar{S} \in \Lambda(\alpha N)$ a fração $z(\bar{S}):=(\bar{S}+1) / 2$ indica a proporção de +1 associada com a média empírica $\bar{S}$.

Aplicando um resultado para grandes desvios de medias empíricas de variáveis aleatórias independentes e identicamente distribuídas que assumem um número finito de valores (veja Dembo e Zeitouni [39], Capítulo 2, Lema 2.1.9) concluímos a primeira desigualdade de

$$
\begin{aligned}
\mu_{1}\left(B_{0}(\bar{S}) \mid w_{t}^{*}\right) & \leq \exp \left\{-\alpha N\left[z(\bar{S}) \log \left(z(\bar{S}) / p^{*}\right)+(1-z(\bar{S})) \log \left((1-z(\bar{S})) /\left(1-p^{*}\right)\right)\right]\right\} \\
& \leq \exp \left\{-\alpha N\left[2\left(p^{*}-z(\bar{S})\right)^{2}\right]\right\} \forall \bar{S} \in \Lambda(\alpha N)
\end{aligned}
$$

A segunda desigualdade pode ser verificada derivando o lado direito da primeira desigualdade 2 vezes em $\bar{S}$.

Como $\bar{S} \in \bar{B}_{\varepsilon-\delta}\left(\bar{S}_{t+1}^{*}\right) \Leftrightarrow\left|p^{*}-z(\bar{S})\right|>(\varepsilon-\delta) / 2$, concluímos finalmente

$$
\begin{aligned}
(\star \star) & \leq\left|\bar{B}_{\varepsilon-\delta}\left(\bar{S}_{t+1}^{*}\right)\right| \exp \left\{-\alpha N\left[2((\varepsilon-\delta) / 2)^{2}\right]\right\} \\
& \leq(\alpha N+1) \exp \left\{-\alpha N[\varepsilon-\delta]^{2} / 2\right\}
\end{aligned}
$$

onde $\left|\bar{B}_{\varepsilon-\delta}\left(\bar{S}_{t+1}^{*}\right)\right|$ denota o número de elementos de $\bar{B}_{\varepsilon-\delta}\left(\bar{S}_{t+1}^{*}\right)$ (no máximo $\left.\alpha N+1\right)$.

Provaremos agora que o lado esquerdo da implicação (2.51) está assegurado para $N$ suficientemente grande, isto é, retomando (2.50), provaremos que existe $N_{t}^{S}(\varepsilon)>0$, tal que, para qualquer $N>N_{t}^{S}(\varepsilon)$, valha

$$
\left|E_{S}\left(w_{t}\right)-E_{S}\left(w_{t}^{*}\right)\right| \leq \Pi_{t+1} / \sqrt[4]{N}<\varepsilon
$$


Demonstração de (2.52). Provaremos inicialmente a primeira desigualdade de (2.52).

Como $G\left(w_{t}\right)=\left(E_{S}\left(w_{t}\right), E_{S}\left(w_{t}\right)\right)$ e $L\left(w_{t}\right)$ é uma constante de Lipschitz local de $G$ no ponto $w_{t}$, temos

$$
\left|E_{S}\left(w_{t}\right)-E_{S}\left(w_{t}^{*}\right)\right| \leq\left\|G\left(w_{t}\right)-G\left(w_{t}^{*}\right)\right\|_{\max } \leq L\left(w_{t}\right)\left\|w_{t}-w_{t}^{*}\right\|_{\max }
$$

desde que $\left\|w_{t}-w_{t}^{*}\right\|_{\max } \leq \vartheta\left(w_{t}\right)$. Como $w_{t}^{*} \in B_{\varepsilon_{t}(N)}\left(w_{t}\right)$, existe $N_{t}^{S}>0$ suficientemente grande, tal que, para $N>N_{t}^{S}$, vale

$$
\left\|w_{t}-w_{t}^{*}\right\|_{\max } \leq \varepsilon_{t}(N)=\frac{\Pi_{t+1}}{L\left(w_{t}\right) \sqrt[4]{N}}<\vartheta\left(w_{t}\right)
$$

Portanto, (2.53) está satisfeita para $N>N_{t}^{S}$. Agora, como para $N>N_{t}^{S}$ vale (2.53) e (2.54), podemos substituir $\left\|w_{t}-w_{t}^{*}\right\|_{\text {max }}$ por $\Pi_{t+1} /\left(L\left(w_{t}\right) \sqrt[4]{N}\right)$ em (2.53) e concluir a validade da primeira desigualdade de (2.52) para $N_{t}>N_{t}^{S}$.

A segunda desigualdade de (2.52) está garantida para $N$ maior que um certo $N_{t}(\varepsilon)$, pois $\Pi_{t+1}$ é positivo e não depende de $N$. Podemos assumir $N_{t}(\varepsilon)>N_{t}^{S}$ e concluir que ambas as desigualdades de (2.52) valem para $N>N_{t}(\varepsilon)$.

Com a prova de (2.52), a prova de (2.49) está concluída.

De maneira análoga a (2.49), podemos estimar $\mu_{1}\left(\bar{B}_{\varepsilon}\left(\bar{F}_{t+1}^{(\infty)}\right) \mid w_{t}^{*}\right)$. Ou seja, para todo $\varepsilon$, existe $N_{t}^{F}(\varepsilon)>0$, tal que, para todo $N>N_{t}^{F}(\varepsilon)$, vale

$$
\begin{cases}\mu_{1}\left(\bar{B}_{\varepsilon}\left(\bar{F}_{t+1}^{(\infty)}\right) \mid w_{t}^{*}\right)=0 & \text { se } 1-\alpha=0 \\ \mu_{1}\left(\bar{B}_{\varepsilon}\left(\bar{F}_{t+1}^{(\infty)}\right) \mid w_{t}^{*}\right) \leq((1-\alpha) N+1) \exp \left\{-\frac{1}{2}(1-\alpha) N[\varepsilon-\delta]^{2}\right\} & \text { se } 1-\alpha>0\end{cases}
$$

Levando em conta (2.48), (2.49), (2.55) e que $\alpha \in[0,1]$, temos que 
para $N>N_{t}(\varepsilon):=\operatorname{Max}\left\{N_{t}^{h}(\varepsilon), N_{t}^{S}(\varepsilon), N_{t}^{F}(\varepsilon)\right\}$, vale

$$
\begin{gathered}
\mu_{1}\left(\bar{B}_{\varepsilon}\left(w_{t+1}\right) \mid w_{t}^{*}\right) \\
\leq \\
(\alpha N+1) \exp \left\{-\frac{1}{2} \underline{\alpha} N[\varepsilon-\delta]^{2}\right\}+((1-\alpha) N+1) \exp \left\{-\frac{1}{2} \underline{\alpha} N[\varepsilon-\delta]^{2}\right\} \\
\leq \\
(N+1) \exp \left\{-\frac{1}{2} \underline{\alpha} N[\varepsilon-\delta]^{2}\right\}+2(N+1) \exp \left\{-\frac{1}{2} \underline{\alpha} N[\varepsilon-\delta]^{2}\right\} \\
\leq \\
(N+1) \exp \left\{-\frac{1}{2} \underline{\alpha} N[\varepsilon-\delta]^{2}\right\} \\
= \\
2(N+1) \exp \left\{-\frac{1}{2} \underline{\alpha} N\left[\varepsilon-\Pi_{t+1} / \sqrt[4]{N}\right]^{2}\right\}
\end{gathered}
$$

onde $\underline{\alpha}:=\operatorname{Min}\{\alpha, 1-\alpha\}$ se $0<\alpha<1$ e $\underline{\alpha}:=1$ se $\alpha=0$ ou $\alpha=1$.

Com esse último, passo a prova de (2.44) está concluída.

Demonstração de (2.45). A prova se dará por indução.

Início da indução: para $t=0$ vale (2.45).

Demonstração. Conforme (2.42) e (2.25), $\varepsilon_{0}(N)=1 / \sqrt[4]{N}$ e, portanto, para qualquer $\varepsilon>0$ e $N>0$ vale $\mu_{0}\left(\bar{B}_{\varepsilon_{0}(N)}\left(w_{0}\right) \mid w_{0}\right)=0 \leq \varepsilon_{0}(N)$.

Passo da indução: supondo $t>0$ e que (2.45) vale para $t-1$, temos que (2.45) vale para $t$.

Demonstração. Como (2.41) vale para qualquer $\varepsilon>0$ e $t>0$, podemos escolher $\varepsilon=\varepsilon_{t}(N)$ e afirmar inicialmente que, para $N>0$ e $t>0$, vale

$$
\mu_{t}\left(\bar{B}_{\varepsilon_{t}(N)}\left(w_{t}\right) \mid w_{0}\right) \leq \mu_{t-1}\left(\bar{B}_{\varepsilon_{t-1}(N)}\left(w_{t}\right) \mid w_{0}\right)+\mu_{1}\left(\bar{B}_{\varepsilon_{t}(N)}\left(w_{t}\right) \mid w_{t-1}^{*}\right)
$$

onde $w_{t-1}^{*} \in B_{\varepsilon_{t-1}(N)}\left(w_{t-1}\right)$ e $w_{t-1}^{*}$ definido de tal sorte a maximizar a probabilidade condicional $\mu_{1}\left(\bar{B}_{\varepsilon_{t}(N)}\left(w_{t}\right) \mid w_{t-1}^{*}\right)$, i.e.,

$$
\mu_{1}\left(\bar{B}_{\varepsilon_{t}(N)}\left(w_{t}\right) \mid w_{t-1}^{*}\right)=\operatorname{Max}\left\{\mu_{1}\left(\bar{B}_{\varepsilon_{t}(N)}\left(w_{t}\right) \mid w\right): w \in B_{\varepsilon_{t-1}(N)}\left(w_{t-1}\right)\right\}
$$

O objetivo final do próximo passo é estimar $\mu_{1}\left(\bar{B}_{\varepsilon_{t}(N)}\left(w_{t}\right) \mid w_{t-1}^{*}\right)$ da mesma forma que estimamos (2.44), i.e., substituindo em (2.44) $t$ por $t-1$ e $\varepsilon$ por $\varepsilon_{t}(N)$. Para que isso 
possa ser feito, é necessário assegurar somente o equivalente a (2.52), ou seja, que existe $N_{t}$, tal que, para $N>N_{t}$, valha

$$
\left|E_{S}\left(w_{t-1}\right)-E_{S}\left(w_{t-1}^{*}\right)\right| \leq \Pi_{t} / \sqrt[4]{N}<\varepsilon_{t}(N)
$$

Com exceção da prova de (2.58), todas os demais argumentos empregados para a estimativa de $\mu_{1}\left(\bar{B}_{\varepsilon_{t}(N)}\left(w_{t}\right) \mid w_{t-1}^{*}\right)$ são os mesmos já empregados na prova de (2.44).

Por enquanto, vamos supor que (2.58) esteja assegurado para $N>N_{t}$ (provaremos mais adiante a validade de (2.58) para $N>N_{t}$ ). Sob esta suposição, podemos concluir que, para $N>N_{t}$, vale

$$
\begin{aligned}
\mu_{1}\left(\bar{B}_{\varepsilon_{t}(N)}\left(w_{t}\right) \mid w_{t-1}^{*}\right) & \leq 2(N+1) \exp \left\{-\frac{1}{2} \underline{\alpha} N\left[\varepsilon_{t}(N)-\Pi_{t} / \sqrt[4]{N}\right]^{2}\right\} \\
& =2(N+1) \exp \left\{-\frac{1}{2} \underline{\alpha} N\left[\Pi_{t+1} /\left(L\left(w_{t}\right) \sqrt[4]{N}\right)-\Pi_{t} / \sqrt[4]{N}\right]^{2}\right\} \\
& =2(N+1) \exp \left\{-\frac{1}{2} \underline{\alpha} N\left[\left(e^{c}-1\right) \Pi_{t} / \sqrt[4]{N}\right]^{2}\right\} \\
& =2(N+1) \exp \left\{-\frac{1}{2} \underline{\alpha}\left[\left(e^{c}-1\right) \Pi_{t}\right]^{2} \sqrt{N}\right\}
\end{aligned}
$$

onde $\underline{\alpha}:=\operatorname{Min}\{\alpha, 1-\alpha\}$ se $0<\alpha<1$ e $\underline{\alpha}:=1$ se $\alpha=0$ ou $\alpha=1$.

Vamos agora substituir em (2.56) a probabilidade condicional $\mu_{1}\left(\bar{B}_{\varepsilon_{t}(N)}\left(w_{t}\right) \mid w_{t-1}^{*}\right)$ pela estimativa acima obtida e substituir também $\mu_{t-1}\left(\bar{B}_{\varepsilon_{t-1}(N)}\left(w_{t}\right) \mid w_{0}\right)$ pela hipótese de indução. Assim, para $N>N_{t}$, vale

$$
\begin{gathered}
\mu_{t}\left(\bar{B}_{\varepsilon_{t}(N)}\left(w_{t}\right) \mid w_{0}\right) \\
\leq \\
2(t-1)(N+1) \exp \left\{-\frac{1}{2} \underline{\alpha} M_{t-1} \sqrt{N}\right\}+2(N+1) \exp \left\{-\frac{1}{2} \underline{\alpha}\left[\left(e^{c}-1\right) \Pi_{t}\right]^{2} \sqrt{N}\right\}
\end{gathered}
$$

onde $\underline{\alpha}:=\operatorname{Min}\{\alpha, 1-\alpha\}$ se $0<\alpha<1$ e $\underline{\alpha}:=1$ se $\alpha=0$ ou $\alpha=1$.

Agora, como pela definição (2.26) $M_{t-1}=\left[\left(e^{c}-1\right) \operatorname{Min}_{0 \leq \kappa \leq t-1}\left(\Pi_{\kappa}\right)\right]^{2}$, concluímos finalmente que, para $N>N_{t}$, vale

$$
\mu_{t}\left(\bar{B}_{\varepsilon_{t}(N)}\left(w_{t}\right) \mid w_{0}\right) \leq 2 t(N+1) \exp \left\{-\frac{1}{2} \underline{\alpha} M_{t} \sqrt{N}\right\}
$$

onde $\underline{\alpha}:=\operatorname{Min}\{\alpha, 1-\alpha\}$ se $0<\alpha<1$ e $\underline{\alpha}:=1$ se $\alpha=0$ ou $\alpha=1$.

Com esse passo, acabamos de provar (2.45) sob a suposição de que (2.58) esteja assegurado para $N>N_{t}$. 
Demonstração de (2.58). Provaremos que existe $N_{t}$, tal que, para todo $N>N_{t}$, valha (2.58). A validade da primeira desigualdade de (2.58) para $N$ suficientemente grande já foi provada com a prova de (2.52). A segunda desigualdade vale para qualquer $N>0$. Este fato decorre da seguinte relação:

$$
\Pi_{t} / \sqrt[4]{N}<e^{c} \Pi_{t} / \sqrt[4]{N}=\Pi_{t+1} /\left(L\left(w_{t}\right) \sqrt[4]{N}\right)=\varepsilon_{t}(N)
$$

Com esse último passo, concluímos a prova de (2.45) e fechamos a prova da Proposição 1. 


\subsection{Dinâmica de grandes populações}

A seguir, estudaremos as propriedades do sistema dinâmico $\left(w_{t}\right)_{t \geq 0}$ definido em (2.20) para as probabilidades de compra dadas em (2.16). ${ }^{4}$ Mais especificamente, deduziremos o comportamento do sistema dinâmico $\left(w_{t}\right)_{t \geq 0}$ para diferentes regiões dos parâmetros $\Phi$ (distribuição das avaliações individuais sobre o valor fundamental do ativo), $J$ (influência social de preço), $K$ (influência social direta) e $\alpha$ (fração de especuladores no mercado). Observamos que o objetivo principal do trabalho é a análise do comportamento do processo estocástico do preço $\left(P_{t}\right)_{t \geq 0}$ em função dos parâmetros mencionados acima.

A relação do estudo das propriedades do sistema dinâmico $\left(w_{t}\right)_{t \geq 0}$ com o processo estocástico do preço $\left(P_{t}\right)_{t \geq 0}$ vem da Proposição 1 . A Proposição 1 afirma que o processo estocástico construído (aquele que designamos por $\left(W_{t}\right)_{t \geq 0}$ ) converge para o processo determinístico $\left(w_{t}\right)_{t \geq 0}$. Portanto, para uma grande quantidade de agentes e para um horizonte de tempo finito, podemos aproximar a evolução do preço $P_{t}$ pelo limite de grandes populações do preço $P_{t}^{(\infty)}$. Por sua vez, $P_{t}^{(\infty)}=H_{t}^{(\infty)}+\bar{P}$, sendo que $H_{t}^{(\infty)}$ é a primeira componente de $w_{t}$.

Para podermos aplicar a Proposição 1 às probabilidades de compra dadas em (2.16), é necessário verificar a validade das suposições da Proposição 1 neste caso, i.e, para as probabilidades de compra dadas em (2.16). Esta verificação é fácil. Faremos isso logo adiante. Inicialmente, gostaríamos de comentar as razões que nos levaram a estruturar a presente seção da forma como é apresentada. Como será visto, a seção aborda o comportamento do processo $\left(w_{t}\right)_{t \geq 0}$ para diferentes regiões dos parâmetros do modelo. Os casos estudados correspondem a situações realistas diferentes e também exibem propriedades matemáticas diferentes. Notamos que, em cada caso, a estimativa da probabilidade de desvio entre o processo $\left(W_{t}\right)_{t \geq 0}$ e o sistema dinâmico $\left(w_{t}\right)_{t \geq 0}$ pode ser melhorada via aplicação dos Corolários 1 e 2 da Proposição 1. Estes melhoramentos serão indicados e discutidos no decorrer do texto.

Conforme anunciamos no parágrafo anterior, mostraremos agora que nosso processo original $\left(W_{t}\right)_{t \geq 0}$ converge para o processo $\left(w_{t}\right)_{t \geq 0}$ quando o número de agentes (parâmetro

\footnotetext{
${ }^{4}$ Lembramos que estas probabilidades de compra foram deduzidas a partir das regras (2.9) e (2.12). No Capítulo 3 modificaremos estas regras. Porém, estas modificações não exigirão grandes mudanças, pois a Proposição 1 e seus corolários foram feitos para serem facilmente aplicados às novas probabilidades de compra que originaram das novas regras de compra e venda que apresentaremos no Capítulo 3.
} 
do processo $\left.\left(W_{t}\right)_{t \geq 0}\right)$ tende ao infinito.

O que precisamos fazer é verificar a validade das suposições da Proposição 1 para a probabilidade de compra de especuladores $Q_{S}$ e para a probabilidade de compra de fundamentalistas $Q_{F}$ dadas em (2.16). Lembramos que $Q_{S}$ e $Q_{F}$ foram dadas da seguinte forma em (2.16): para cada $w:=(H, \bar{S}, \bar{F}) \in \mathbb{R} \times[-1,1]^{2}$, tínhamos

$$
Q_{S}(w)=\Phi(J \bar{A}+K \bar{S}-(H+\lambda \bar{A})) \quad \text { e } \quad Q_{F}(w)=\Phi(-(H+\lambda \bar{A}))
$$

onde $\bar{A}=\alpha \bar{S}+(1-\alpha) \bar{F}$.

Como todas as derivadas parciais das probabilidades de compra $Q_{S}(w)$ e $Q_{F}(w)$ dadas em (2.59) existem, isto é, não são infinitas, temos que $G$ possui constantes de Lipschitz locais no sentido (2.22) em todo o seu domínio de definição $\mathbb{R} \times[-1,1]^{2}$. Lembramos que $G: \mathbb{R} \times[-1,1]^{2} \rightarrow[-1,1]$ foi definida por $G(w):=\left(E_{S}(w), E_{F}(w)\right)$, onde

$$
E_{S}(w)=\left\{\begin{array}{ll}
2 Q_{S}(w)-1 & \text { se } \alpha>0 \\
0 & \text { caso contrário }
\end{array} \quad E_{F}(w)= \begin{cases}2 Q_{F}(w)-1 & \text { se }(1-\alpha)>0 \\
0 & \text { caso contrário }\end{cases}\right.
$$

onde $\alpha \in[0,1]$.

As constantes de Lipschitz acima mencionadas podem ser dadas da seguinte forma: para cada $w \in \mathbb{R} \times[-1,1]^{2}$, definimos uma constante de Lipschitz local de $G$ em $w$, i.e., que vale para uma vizinhança de $w$, por $L(w):=\left\|G^{\prime}(w)\right\|_{\max }+\gamma$, onde $\gamma>0$ e onde $G^{\prime}(w)$ denota a derivada de $G$ no sentido do Corolário 2 da Proposição 1. Com isso, as condições da Proposição 1 estão satisfeitas e vale a convergência $W_{t} \stackrel{P}{\rightarrow} w_{t}$ (em probabilidade) quando $N \rightarrow \infty$, i.e., quando o número de agentes $N$ tende ao infinito.

\subsubsection{Caso $J \geq 0, K=0$ e $\lambda=0$ ( $H_{t}=h$ não varia no tempo)}

Nesta seção analisaremos a dinâmica de $\left(w_{t}\right)_{t \geq 0}$ quando $K=0$ e $\lambda=0$. Este caso corresponde à situação real na qual os especuladores não se deixam influenciar diretamente pela opinião de outros especuladores $(K=0)$, podendo, no entanto, ser influenciados apenas pela tendência do preço $(J \geq 0)$, porém, sob a suposição de que a sobrevalorização do ativo não varie no tempo $(\lambda=0) .^{5}$

\footnotetext{
${ }^{5}$ Lembramos ao leitor que segundo a fórmula (2.12), $J$ foi definido por $J:=J_{1} \lambda / J_{3} \geq 0$. É claro que desta definição segue necessariamente $\lambda=0 \Rightarrow J=0$. No entanto, queremos considerar o sistema dinâmico $\left(w_{t}\right)_{t \geq 0}$ dado em (2.20), onde $\lambda$ é substituído por uma outra constante $\lambda^{\prime}>0$ desvinculada de $J$ (variação da sobrevalorização do ativo desvinculada da variação do preço). Para evitar a introdução
} 


\subsubsection{Justificativa do interesse no caso $\lambda=0$}

A particularidade desta (e de várias outras seções) está no estudo do comportamento do sistema dinâmico $\left(w_{t}\right)_{t \geq 0}$ quando $\lambda=0$. Lembramos que o estudo de $\left(w_{t}\right)_{t \geq 0}$ tem o objetivo de revelar o comportamento do processo original $\left(W_{t}\right)_{t \geq 0}$, onde $\lambda>0$. Deste fato surge naturalmente a questão sobre as razões de considerarmos o caso $\lambda=0$. Explicaremos nosso interesse no caso $\lambda=0$ a seguir. Antes, porém, faremos algumas considerações que serão usadas no tratamento do caso $\lambda=0$.

Note inicialmente que, sob $\lambda=0$, o sistema dinâmico $\left(w_{t}\right)_{t \geq 0}$ corresponde ao sistema dinâmico definido em (2.20), onde

$$
H_{t}=h:=H_{0}, \quad \forall t \geq 0
$$

e onde $Q_{S}$ e $Q_{F}$ são dadas segundo (2.59). Portanto, no caso $\lambda=0$, podemos definir as probabilidades de compra de especuladores e fundamentalistas, $Q_{S}$ e $Q_{F}$, como sendo funções constantes em $H$. Isto é, para $w=(H, \bar{S}, \bar{F}) \in \mathbb{R} \times[-1,1]^{2}$, podemos definir

$$
Q_{S}(w)=\Phi(J \bar{A}+K \bar{S}-h) \quad \text { e } \quad Q_{F}(w)=\Phi(-h)
$$

onde $h:=H_{0}$. Note que, quando $\lambda=0$, tanto a definição (2.59) quanto (2.60) resultam no mesmo processo estocástico $\left(W_{t}\right)_{t \geq 0}$ e no mesmo sistema dinâmico $\left(w_{t}\right)_{t \geq 0}$ definidos no enunciado da Proposição 1. Por conveniência ao tratamento que daremos neste caso, optaremos pela definição (2.60) sempre que neste capítulo valer $\lambda=0$.

Como havíamos anunciado, apresentaremos agora as razões que nos levaram a considerar o caso $\lambda=0$, ou seja, o caso $H_{t}=h$ ( $h$ constante). A primeira razão é bastante formal, porém, suficiente para a justificativa do estudo do caso $H_{t}=h$ ( $h$ constante). A razão é que este estudo facilitará o estudo do processo original $\left(w_{t}\right)_{t \geq 0}$ no qual $\lambda>0$, i.e., no qual $H_{t}$ varia no tempo. Mais especificamente, ocorre que as propriedades do equilíbrio de excesso de demanda no caso $H_{t}=h$ ( $h$ const.), que serão reveladas na presente seção, serão de grande utilidade para o estudo do comportamento de $\left(w_{t}\right)_{t \geq 0}$ no caso $\lambda>0$.

A segunda razão é que o caso $H_{t}=h$ ( $h$ const.) possui um paralelo na vida real. A fim de descrever este paralelo, observemos inicialmente que o processo estocástico $\left(W_{t}\right)_{t \geq 0}$, de uma segunda constante $\lambda^{\prime}$, vamos considerar o sistema dinâmico (2.20), onde as constantes $\lambda$ e $J$ são desvinculadas uma da outra; podendo valer inclusive $J>0$ e $\lambda=0$. O propósito deste estudo será esclarecido ao justificarmos o interesse no caso $\lambda=0$. 
sob $\lambda=0$, como acima descrito, pode ser obtido se considerarmos $\lambda>0$ e postularmos que a avaliação média sobre o valor fundamental do ativo (não seja uma constante $\bar{P}$, mas sim que a mesma) seja um processo $\left(\bar{P}_{t}\right)_{t \geq 0}$ que acompanha o processo de preço a menos de uma defasagem constante $h$, ou seja, $\bar{P}_{t}:=P_{t}-h$ ( $h$ const. $), t=0,1,2, \ldots$ Acreditamos que esta situação ocorra na vida real quando agentes financeiros tomam a própria evolução do preço como um indicador da evolução sobre o valor fundamental do ativo, a menos de uma defasagem $h$. A diferença $h$ continua correspondendo ao quanto os agentes financeiros, em média, acreditam que o preço esteja acima do valor fundamental do ativo $\left(h=P_{t}-\bar{P}_{t}\right)$. Porém, neste caso, uma elevação do preço não aumenta a sobrevalorização do ativo dada por $h$. Sob a ótica deste modelo, onde $\bar{P}_{t}:=P_{t}-h$ ( $h$ const.), uma alteração da defasagem $h$ ocorreria na vida real devido à incidência de novas informações sobre alterações nos fundamentos econômicos do ativo. Sob a ótica desta modelagem, estas notícias chegariam ao mercado de tempos em tempos alterando a percepção média da sobrevalorização do ativo $h$ independente da evolução do preço $P_{t}$.

Existem outros modelos que trabalham com uma diferença constante de preços (no nosso caso, diferença entre $P_{t}$ e $\bar{P}$ ) e que, em essência, analisam o mesmo tipo de fenômeno socioeconômico apresentado nesta seção. Recomendamos o artigo [4] para uma melhor apreciação das análises que apresentaremos sempre que considerarmos $H_{t}=h$ ( $h$ const.). Neste artigo, a situação econômica abordada trata de um conjunto de consumidores que costumam freqüentar dois estabelecimentos distintos cuja diferença de preço de ingresso é $h$ ( $h$ const.). A decisão de qualquer consumidor continuar freqüentando um dos estabelecimentos depende das decisões dos demais consumidores e da diferença $h$ de preços entre os dois estabelecimentos. O problema aqui é determinar até quanto um dado estabelecimento pode aumentar o preço de ingresso e, ao mesmo tempo, garantir que todos os lugares sejam vendidos. Dependendo do contexto, o preço máximo possível pressupõe um certo nível de excesso de demanda (fila). Qualquer preço acima deste preço corresponde a um alto nível de excesso de oferta (subocupação do estabelecimento). 


\subsubsection{Dinâmica de excesso de demanda $\left(\bar{A}_{t}\right)_{t \geq 0}$}

Estudaremos agora a dinâmica da seqüência $\left(\bar{A}_{t}\right)_{t \geq 0}$ para $H_{t}=h, \forall t \geq 0$. Levando em conta as probabilidades de compra (2.59), esta dinâmica é dada pela seguinte recursão

$$
\bar{A}_{t}=g\left(\bar{A}_{t-1} ; h\right), \quad t=1,2, \ldots
$$

onde

$$
g(x ; h):=\alpha[2 \Phi(J x-h)-1]+(1-\alpha)[2 \Phi(-h)-1]
$$

Neste caso particular $(\lambda=0)$, observamos que a dinâmica de $\left(w_{t}\right)_{t \geq 0}$ é unicamente determinada pela dinâmica de $\left(\bar{A}_{t}\right)_{t \geq 0}$. Isto porque $H_{t}=h, \bar{F}_{t}=2 \Phi(-h)-1$ e $\bar{S}_{t}=$ $\left[\bar{A}_{t}-(1-\alpha)(2 \Phi(-h)-1)\right] / \alpha$ para $\alpha>0$ e $\bar{S}_{t}=0$ para $\alpha=0$.

\subsubsection{Excesso de demanda de equilíbrio}

A seguir, definiremos o "excesso de demanda de equilíbrio", que é o objeto principal desta seção. Antes, porém, vamos introduzir a notação $g^{(t)}(x ; h)$ para designar a $t$-ésima iṇteração de $g(\cdot ; h)$ em $x$. Isto é, definimos $g^{(t)}:[-1,1] \times \mathbb{R} \rightarrow[-1,1]$ por

$$
g^{(t)}(x ; h)=g^{(t)}\left(g^{(t-1)}(x ; h) ; h\right)
$$

onde $g^{(1)}(x ; h)=g(x ; h)$.

Diremos que $\bar{A} \in[-1,+1]$ é um equilíbrio de excesso de demanda do sistema dinâmico (2.61) ou equilíbrio do sistema dinâmico (2.61) ou, ainda, simplesmente um excesso de demanda de equilibrio se

$$
\bar{A}=g(\bar{A} ; h)
$$

Diferenciaremos os equilíbrios de excesso de demanda entre equilíbrios globalmente estáveis, localmente estáveis e equilíbrios instáveis, como segue:

1. $\bar{A} \in[-1,+1]$ é globalmente estável se, e somente se, para qualquer $\bar{A}_{0} \in[-1,+1]$, valer $\lim _{t \rightarrow \infty} g^{(t)}\left(\bar{A}_{0} ; h\right)=\bar{A}$;

2. $\bar{A} \in[-1,+1]$ é localmente estável se, e somente se existir um $\varepsilon>0$, tal que, para qualquer $\bar{A}_{0} \in(\bar{A}-\varepsilon, \bar{A}+\varepsilon) \cap[-1,+1]$, valha $\lim _{t \rightarrow \infty} g^{(t)}\left(\bar{A}_{0} ; h\right)=\bar{A}$; 


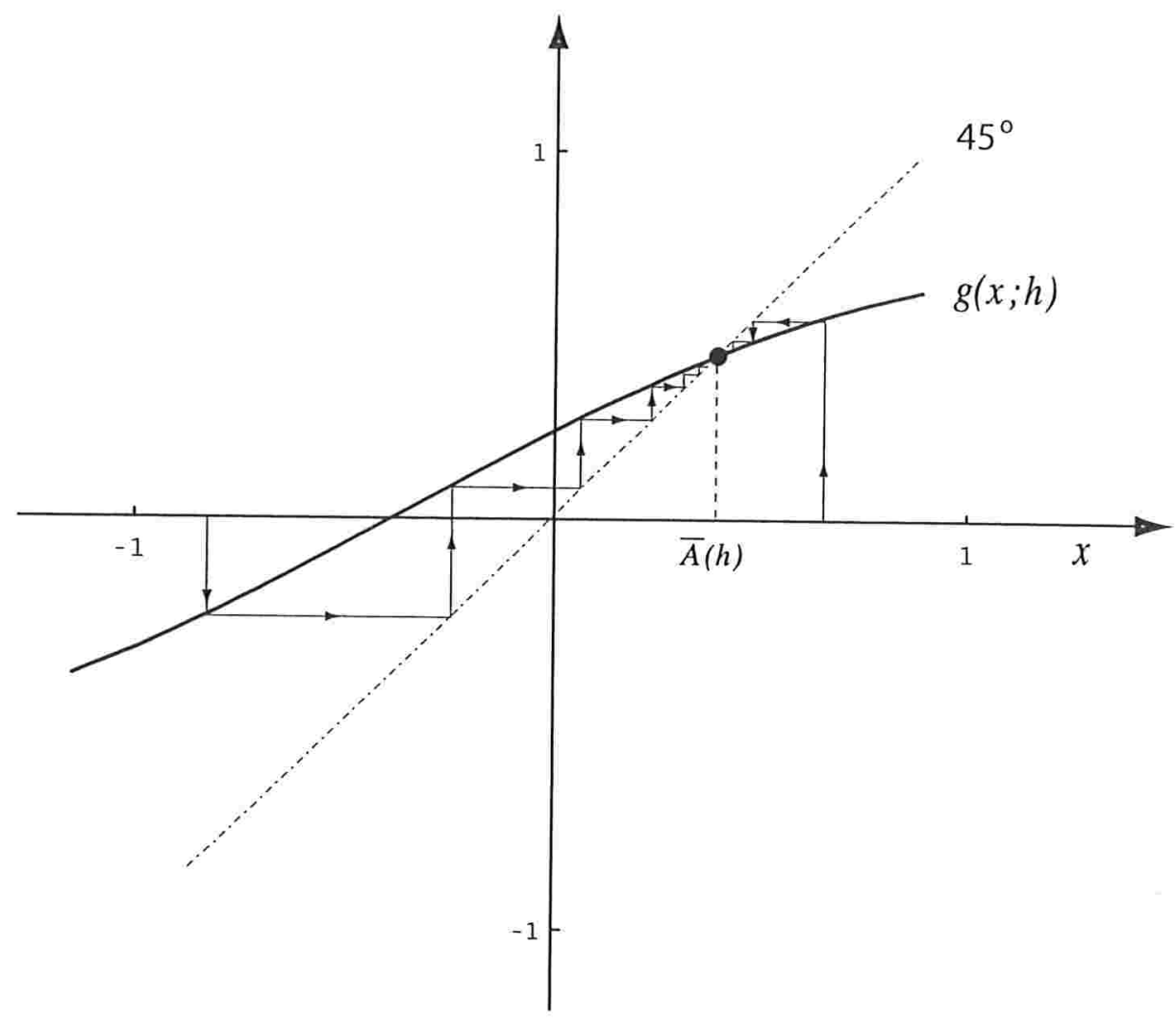

Figura 2.1: $\bar{A}(h)$ é um equilíbrio globalmente estável do sistema dinâmico $\bar{A}_{t+1}=g\left(\bar{A}_{t} ; h\right)$.

3. $\bar{A} \in[-1,+1]$ é um equilíbrio instável se, e somente se, $\bar{A}$ não for nem globalmente estável nem localmente estável.

Definimos o domínio de atração de um equilíbrio $\bar{A}$ de (2.61) como o conjunto dado por

$$
D(\bar{A})=\left\{\bar{A}_{0} \in[-1,1]: \lim _{t \rightarrow \infty} g^{(t)}\left(\bar{A}_{0} ; h\right)=\bar{A}\right\}
$$

Veja as figuras 2.1 e 2.2 .

Chamamos a atenção para fato do conceito de equilíbrio de excesso de demanda não ser equivalente ao conceito de equilíbrio de mercado (onde oferta e demanda do ativo coincidem, i.e., excesso de demanda zero). Somente quando o equilíbrio de excesso de demanda for zero, teremos um equilíbrio de mercado. Ou seja, em geral, o sistema de excessos de demanda dado em (2.61) permite a existência de equilíbrios de excesso de demanda localmente estáveis diferentes de zero. É justamente este fato que, no contexto do modelo, será usado mais adiante para a definição e explicação de bolhas especulativas no mercado financeiro. 


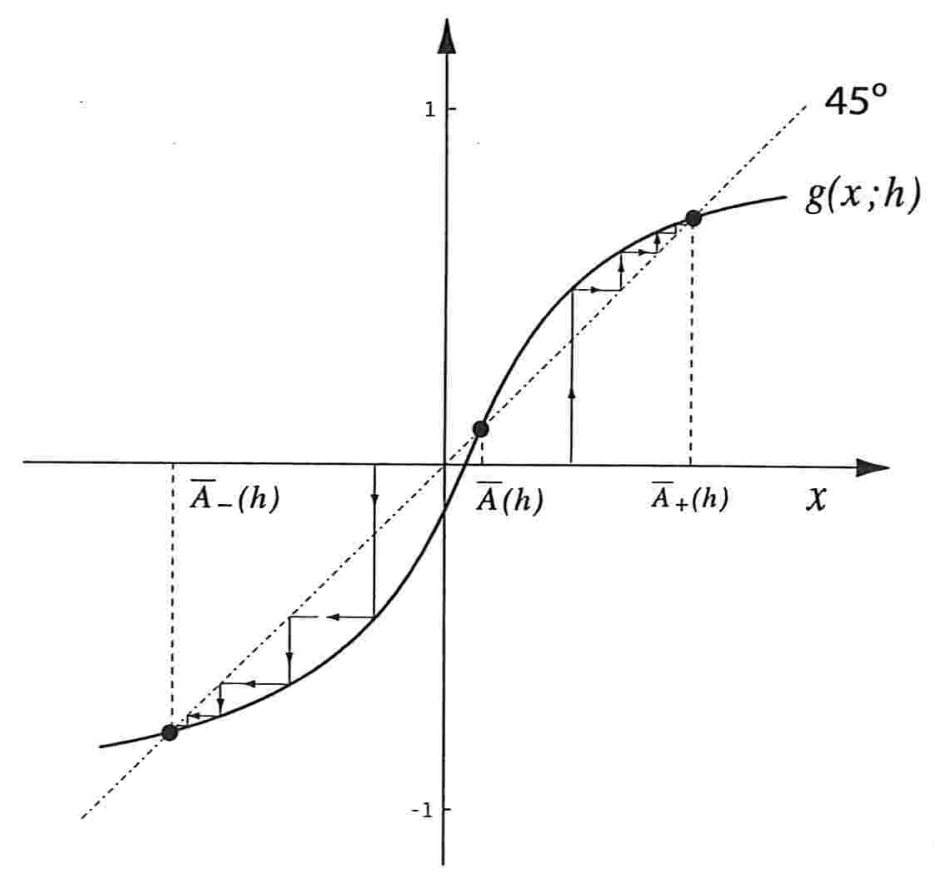

Figura 2.2: $\bar{A}_{-}(h)$ e $\bar{A}_{+}(h)$ são equilíbrios localmente estáveis, enquanto $\bar{A}(h)$ é um equilíbrio instável do sistema dinâmico $\bar{A}_{t+1}=g\left(\bar{A}_{t} ; h\right)$. $[-1, \bar{A}(h))$ e $\left.(\bar{A}(h)), 1\right]$ são os domínios de atração de $\bar{A}_{-}(h)$ e $\bar{A}_{+}(h)$, respectivamente.

\subsubsection{O resultado principal: transição de fase entre excessos de demanda de equilíbrio}

$\mathrm{Na}$ seção anterior, definimos os excessos de demanda de equilíbrio e classificamos estes equilíbrios em equilíbrios globalmente estáveis, localmente estáveis e equilíbrios instáveis. Nesta seção, estudaremos os equilíbrios do sistema de excesso de demanda $\bar{A}_{t+1}=g\left(\bar{A}_{t} ; h\right)$ em função dos parâmetros da função $g(\cdot ; h)$ definida em (2.62). Apresentaremos os resultados deste estudo formalmente na Proposição 2. Antes, porém, faremos algumas observações que facilitarão a compreensão da Proposição 2 como um todo. Com estas observações, esclareceremos também as razões que nos levaram a apresentar a Proposição 2 da forma como será visto mais adiante.

Observamos que o gráfico da função $g(\cdot ; 0)$ tem o mesmo formato em "S" do gráfico da função $\Phi$ (o formato em " $\mathrm{S}$ " do gráfico de $\Phi$ decorre das propriedades da derivada $\Phi^{\prime}$ apresentadas em (2.5): $\Phi^{\prime}$ é simétrica em torno do zero e estritamente decrescente em $[0, \infty))$. É fácil ver que $g(\cdot ; 0)$ possui três pontos fixos se, e somente se, $g^{\prime}(0 ; 0)>1$, i.e., 


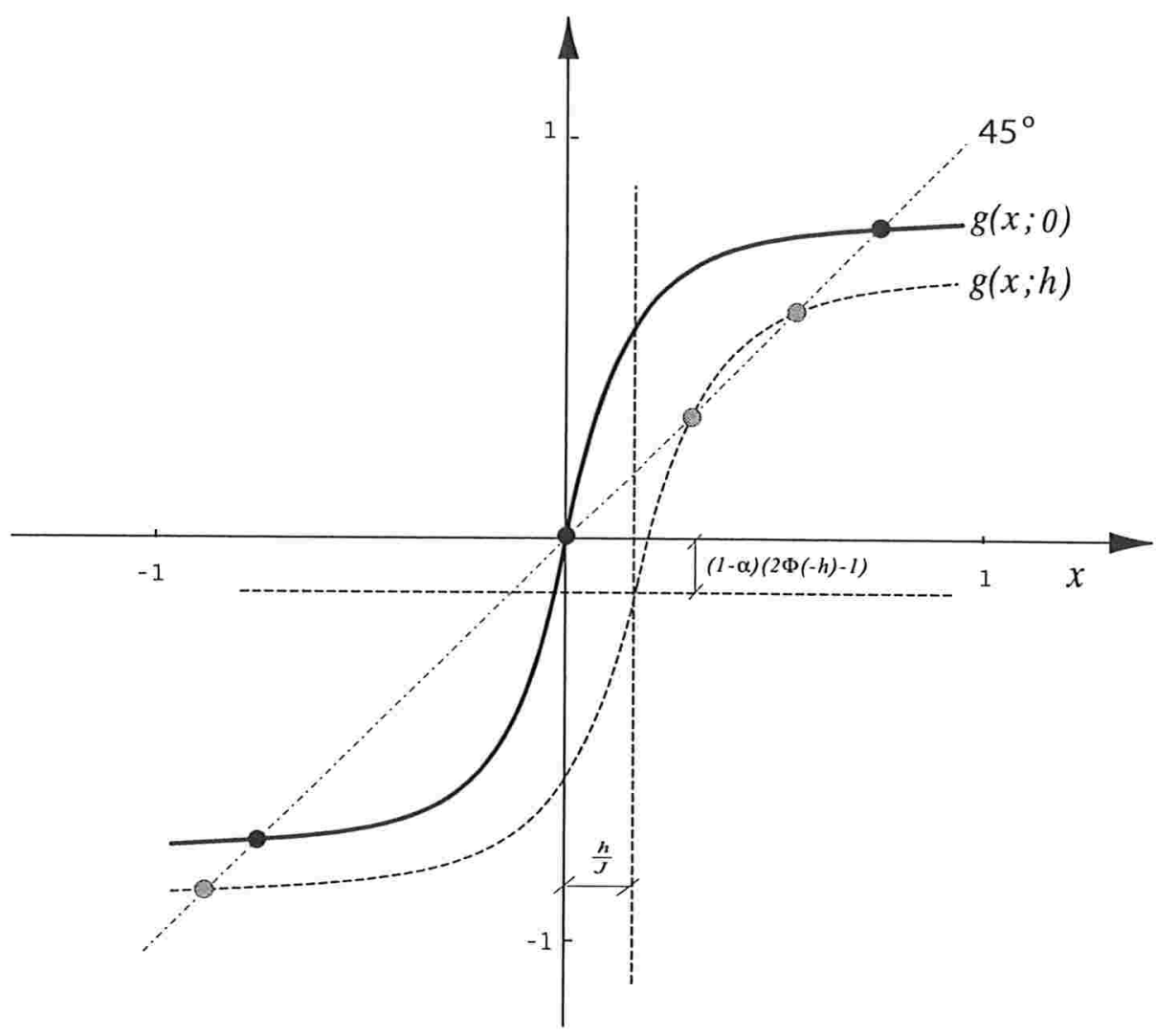

Figura 2.3: O gráfico de $g(\cdot ; h), 0<h<h_{*}$, é uma translação do gráfico de $g(\cdot ; 0)$.

se, e somente se,

$$
2 \alpha J \Phi^{\prime}(0)=\left.\frac{\partial g(x ; 0)}{\partial x}\right|_{x=0}>1
$$

caso contrário, $g(\cdot ; 0)$ possui um único ponto fixo. Como o gráfico de $g(\cdot ; h)$ é uma "translação contínua em $h$ " do gráfico de $g(\cdot ; 0)$, o mesmo vale para $g(\cdot ; h)$, desde que $|h|$ não exceda um determinado valor crítico. Ou seja, $g(\cdot ; h)$ possui três pontos fixos se, e somente se, valer (2.66) e $|h|$ for menor que um valor crítico $h_{*}$ (a ser especificado na Proposição 2); caso contrário, $g(\cdot ; h)$ possui um ou dois pontos fixos (veja a Figura 2.3). Notamos que a condição (2.66), necessária para a ocorrência de mais de um excesso de demanda de equilíbrio (pontos fixos de $g(\cdot ; h)$ ), pode ser expressa da seguinte forma:

$$
\left(\Phi^{\prime}(0)\right)^{-1}<2 \alpha J
$$


Na Proposição 2, usaremos (2.67) para formular a condição necessária para que ocorra mais de um equilíbrio de excesso de demanda. Esta formulação visa a uma interpretação socioeconômica que faremos na Seção 2.4.1.5. Como veremos na Seção 2.4.1.5, o valor de $\left(\Phi^{\prime}(0)\right)^{-1}$ pode ser interpretado como uma medida de heterogeneidade das avaliações individuais sobre o valor fundamental do ativo. Com base nesta interpretação e na interpretação dos demais parâmetros $(\alpha, J$ e $h$ ), daremos, na Seção 2.4.1.5, uma explicação socioeconômica para a ocorrência de bolhas especulativas e crashes de preço no mercado financeiro.

Dependendo de $\alpha$ (fração de especuladores no mercado), de $J$ (influência social de preço), de $\left(\Phi^{\prime}(0)\right)^{-1}$ (dispersão das avaliações individuais sobre o valor fundamental do ativo) e de $h$ (sobrevalorização do ativo), o sistema (2.61) admitirá no mínimo um e no máximo três equilíbrios de excesso de demanda. As figuras 2.4 e 2.5 ilustram os equilíbrios de excesso de demanda para as diferentes regiões de valores dos parâmetros $\alpha, J, \Phi(0)^{-1}$ e $h$, que serão tratadas na Proposição 2.

Dadas as propriedades de $\Phi$ em (2.5), podemos formalizar as relações ilustradas com as figuras 2.4 e 2.5 como segue abaixo.

Proposição 2. Seja $\Phi$ uma função de distribuição de probabilidades que satisfaz as propriedades de (2.5). Sejam $\alpha \in[0,1], J \in[0, \infty)$ e $h \in \mathbb{R}$ três constantes arbitrárias e seja $g$ como definida em (2.62). Definindo ${ }^{6} M\left(\alpha, J, \Phi^{\prime}(0)^{-1}, h\right):=\{x \in[-1,1] \mid x=g(x ; h)\}$ e denotando o número de elementos de $M\left(\alpha, J, \Phi(0)^{-1}, h\right)$, quer dizer, o número de equilíbrios do sistema (2.61), por $\left|M\left(\alpha, J, \Phi^{\prime}(0)^{-1}, h\right)\right|$, temos as seguintes relações:

1. $S e\left(\Phi^{\prime}(0)\right)^{-1} \geq 2 \alpha J$

então $\left|M\left(\alpha, J, \Phi^{\prime}(0)^{-1}, h\right)\right|=1$; e o único elemento de $M\left(\alpha, J, \Phi^{\prime}(0)^{-1}, h\right)$ é globalmente estável; mantendo os parâmetros $\alpha, J$ e $\Phi$ fixos, porém, arbitrários, e, denotando o único equilibrio de $M\left(\alpha, J, \Phi^{\prime}(0)^{-1}, h\right)$ por $\bar{A}(h)$, temos que $\bar{A}(h)$ é estritamente decrescente, impar $\overline{(A}(0)=0)$ e continuo em $h$ (veja a Figura 2.8).

2. $S e\left(\Phi^{\prime}(0)\right)^{-1}<2 \alpha J$

\footnotetext{
${ }^{6}$ A princípio, $M$ depende de todo $\Phi$; porém, as propriedades de $M$ que estão sendo estudadas na Proposição 2, dependem somente da característica $\left(\Phi^{\prime}(0)\right)^{-1}$ da função $\Phi$. É por isso que escrevemos $M$ como função desta característica e não de $\Phi$ como um todo.
} 


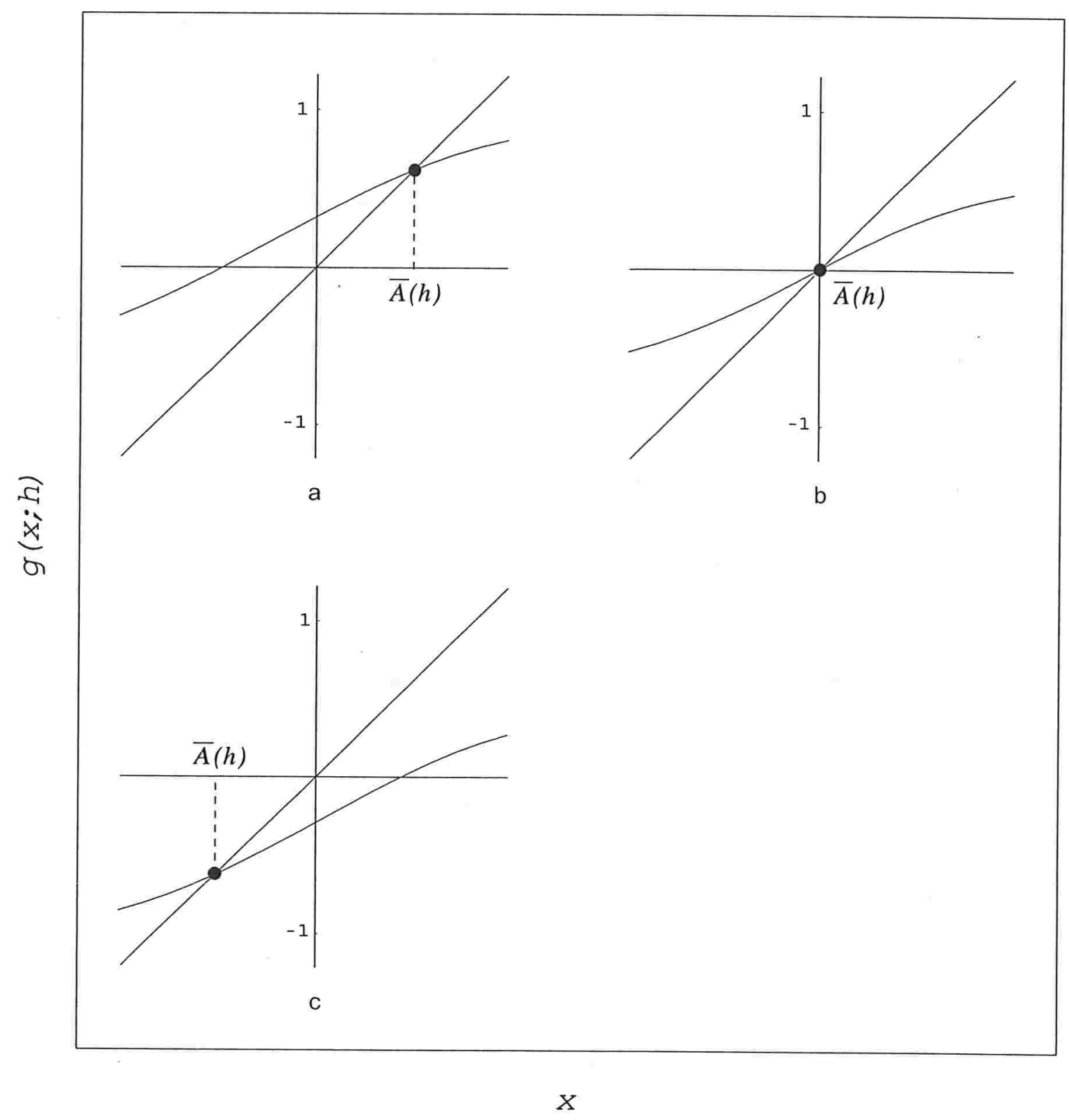

Figura 2.4: Caso $\left(\Phi^{\prime}(0)\right)^{-1} \geq 2 \alpha J$. $\bar{A}(h)$ é o único excesso de demanda de equilíbrio. Subcasos: a) $h<0$; b) $h=0$; e c) $h>0$. 


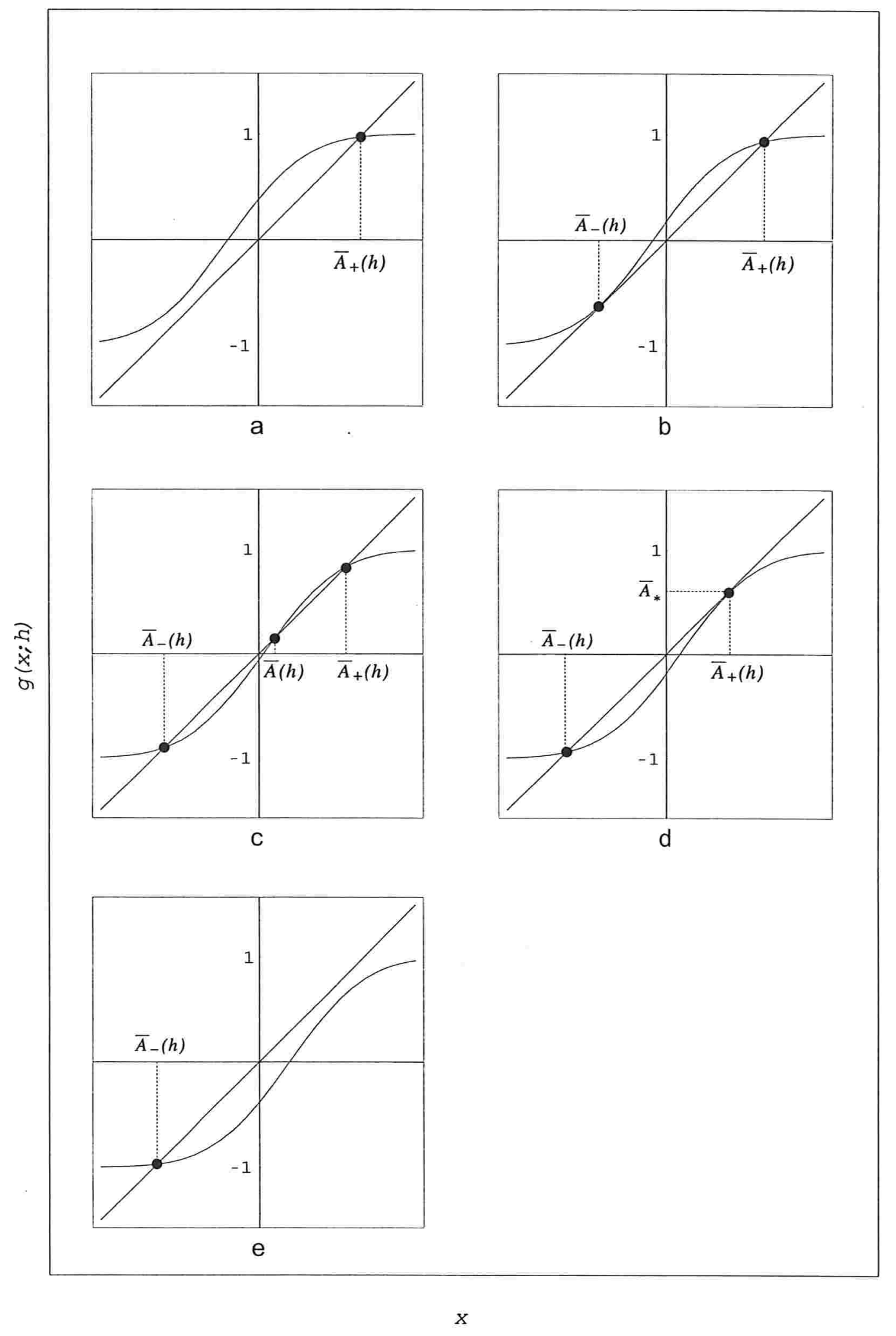

Figura 2.5: Caso $\left(\Phi^{\prime}(0)\right)^{-1}<2 \alpha J . \bar{A}_{-}(h), \bar{A}(h)$ e $\bar{A}_{+}(h)$ são equilíbrios de excesso de demanda. Subcasos: a) $h<-h_{*}$; b) $h=-h_{*}$; c) $-h_{*}<h<h_{*}$; d) $h=h_{*}$; e e) $h>h_{*}$. 
então $\left|M\left(\alpha, J, \Phi^{\prime}(0)^{-1}, h\right)\right|$ pode ser 1,2 ou 3 , dependendo das condições expostas abaixo; estas condições envolvem o par de valores $\left(h_{*}, \bar{A}_{*}\right)>(0,0)$ chamados de sobrevalorização crítica do ativo $e$ excesso de demanda crítico do ativo, respectivamente, definidos pela solução do sistema abaixo que satisfaz $h_{*}>0$ e $\bar{A}_{*}>0$

$$
\left\{\begin{array}{l}
\bar{A}_{*}=\alpha\left[2 \Phi\left(J \bar{A}_{*}-h_{*}\right)-1\right]+(1-\alpha)\left[2 \Phi\left(-h_{*}\right)-1\right] \\
1=2 \alpha \Phi^{\prime}\left(J \bar{A}_{*}-h_{*}\right) J
\end{array}\right.
$$

(a) $S e-\infty<h<-h_{*}$ então $\left|M\left(\alpha, J, \Phi^{\prime}(0)^{-1}, h\right)\right|=1$ e o único elemento de $M\left(\alpha, J, \Phi^{\prime}(0)^{-1}, h\right)$, denotado por $\bar{A}_{+}(h)$, é um equilibrio globalmente estável;

(b) $\mathrm{Se} h=-h_{*}$ então $\left|M\left(\alpha, J, \Phi^{\prime}(0)^{-1}, h\right)\right|=2$ e os dois elementos de $M\left(\alpha, J, \Phi^{\prime}(0)^{-1}, h\right)$, denotados por $\bar{A}_{-}\left(-h_{*}\right)$ e $\bar{A}_{+}\left(-h_{*}\right)$, satisfazem

i. $\bar{A}_{-}\left(-h_{*}\right)<\bar{A}_{+}\left(-h_{*}\right) \operatorname{com} \bar{A}_{-}\left(-h_{*}\right)=-\bar{A}_{*}$;

ii. $\bar{A}_{-}\left(-h_{*}\right)$ é instável e $\bar{A}_{+}\left(-h_{*}\right)$ é localmente estável;

iii. $D\left(\bar{A}_{-}\left(-h_{*}\right)\right)=\left[-1,-\bar{A}_{*}\right]$ e $D\left(\bar{A}_{+}\left(-h_{*}\right)\right)=\left(-\bar{A}_{*}, 1\right]$

(domínios de atração dos equilíbrios);

(c) $S e-h_{*}<h<h_{*}$ então $\left|M\left(\alpha, J, \Phi^{\prime}(0)^{-1}, h\right)\right|=3$ e os três elementos de $M\left(\alpha, J, \Phi^{\prime}(0)^{-1}, h\right)$, denotados por $\bar{A}_{-}(h), \bar{A}(h)$ e $\bar{A}_{+}(h)$, satisfazem

i. $\bar{A}_{-}(h)<-\bar{A}_{*}<\bar{A}(h)<\bar{A}_{*}<\bar{A}_{+}(h)$;

ii. $\bar{A}(h)$ é instável, enquanto que $\bar{A}_{-}(h)$ e $\bar{A}_{+}(h)$ são localmente estáveis;

iii. $D\left(\bar{A}_{-}(h)\right)=[-1, \bar{A}(h))$,

$D(\bar{A}(h))=\{\bar{A}(h)\}$,

$D\left(\bar{A}_{+}(h)\right)=(\bar{A}(h), 1]$

(domínios de atraçâo dos equilibrios);

(d) $\operatorname{Se} h=h_{*}$

então $\left|M\left(\alpha, J, \Phi^{\prime}(0)^{-1}, h\right)\right|=2$ e os dois elementos de $M\left(\alpha, J, \Phi^{\prime}(0)^{-1}, h\right)$, denotados por $\bar{A}_{-}\left(h_{*}\right)$ e $\bar{A}_{+}\left(h_{*}\right)$, satisfazem

i. $\bar{A}_{-}\left(h_{*}\right)<\bar{A}_{+}\left(h_{*}\right) \operatorname{com} \bar{A}_{+}\left(h_{*}\right)=\bar{A}_{*}$; 
ii. $\bar{A}_{-}\left(h_{*}\right)$ é localmente estável e $\bar{A}_{+}\left(-h_{*}\right)$ é instável;

iii. $D\left(\bar{A}_{-}\left(h_{*}\right)\right)=\left[-1, \bar{A}_{*}\right)$ e $D\left(\bar{A}_{+}\left(h_{*}\right)\right)=\left[\bar{A}_{*}, 1\right]$

(domínios de atração dos equilíbrios);

(e) $S e h_{*}<h<\infty$

então $\left|M\left(\alpha, J, \Phi^{\prime}(0)^{-1}, h\right)\right|=1$ e o único elemento de $M\left(\alpha, J, \Phi^{\prime}(0)^{-1}, h\right)$, denotado por $\bar{A}_{-}(h)$, é um equilibrio globalmente estável; e

(f) $\bar{A}_{+}(h)$ e $\bar{A}_{-}(h)$ são funções decrescentes e contínuas para $h$ pertencente a seus respectivos domínios de definição, i.e., para $h$ pertencente a $\left(-\infty, h_{*}\right]$ $e\left[-h_{*}, \infty\right)$, respectivamente. Além disso, vale $1=\bar{A}_{+}(-\infty)>\bar{A}_{+}(h) \geq \bar{A}_{*}>$ $0>-\bar{A}_{*} \geq \bar{A}_{-}(h)>\bar{A}_{-}(\infty)=-1, \forall h \in\left[-h_{*}, h_{*}\right]$ (veja a Figura 2.9).

A prova da Proposição 2 se dá através de uma aplicação do teorema das funções implícitas, onde se leva em conta as propriedades de $\Phi$ apresentadas em (2.5). Esta prova encontra-se no Apêndice.

\subsubsection{Interpretações socioeconômicas: estabilidade global do excesso de de- manda ou formação exógena de bolhas especulativas e crashes}

A seguir, daremos uma interpretação socioeconômica para a quantidade $\left(\Phi^{\prime}(0)\right)^{-1}$ e comentaremos a dinâmica determinística de $\left(\bar{A}_{t}\right)_{t \geq 0}$ do ponto de vista socioeconômico.

Interpretamos $\left(\Phi^{\prime}(0)\right)^{-1}$ como uma medida de heterogeneidade das avaliações individuais sobre o valor fundamental do ativo. A motivação desta interpretação vem do seguinte fato: para uma grande classe de funções de densidade de probabilidades, a quantidade $\left(\Phi^{\prime}(0)\right)^{-1}$ (inverso do valor da função de densidade no zero) é uma função crescente do desvio padrão de qualquer variável aleatória com função de densidade $\Phi^{\prime}$. Como as avaliações individuais sobre o valor fundamental do ativo, $P_{t}^{(i)}, t \geq 0, i \in A$, são variáveis aleatórias independentes e identicamente distribuídas segundo a função de densidade $\Phi^{\prime}$, temos que a quantidade $\left(\Phi^{\prime}(0)\right)^{-1}$ mede a dispersão média de uma amostra (aleatória) de avaliações $P_{t}^{(i)}, i \in A$, feitas pelos agentes financeiros no instante de tempo $t=0,1,2, \ldots$ (para uma grande classe de $\Phi^{\prime} \mathrm{s}$ ). Notamos ainda que, se $\Phi^{\prime}$ for em particular a função de densidade de probabilidades de uma normal de média zero e desvio padrão $\sigma$, então $\left(\Phi^{\prime}(0)\right)^{-1}(=\sqrt{2 \pi} \sigma)$ é diretamente proporcional ao desvio padrão $\sigma$ das avaliações individuais $P_{t}^{(i)}, i \in A$. 
Vamos agora comentar e interpretar a dinâmica do excesso de demanda $\left(\bar{A}_{t}\right)_{t \geq 0}$ do ponto de vista socioeconômico. Para tanto, faremos uso das afirmações da Proposição 2 , bem como da interpretação de $\left(\Phi^{\prime}(0)\right)^{-1}$ enquanto (medida de) heterogeneidade das avaliações individuais sobre o valor fundamental do ativo. Chamaremos os equilíbrios de excesso de demanda localmente estáveis de "atratores" (do sistema dinâmico $\left(\bar{A}_{t}\right)_{t \geq 0}$ ) ou "excessos de demanda estáveis". Chamaremos $J$ de "influência social (de preço)", pois $J$ quantifica a tendência de especuladores alinharem suas atitudes de investimentos à atitude da maioria dos agentes.

Sob alta heterogeneidade das avaliações individuais sobre o valor fundamental do ativo $\left(\left(\Phi^{\prime}(0)\right)^{-1} \geq 2 \alpha J\right)$, o sistema dinâmico de excesso de demanda $\left(\bar{A}_{t}\right)_{t \geq 0}$ possui um único atrator (excesso de demanda de equilíbrio localmente estável) $\bar{A}(h)$. O atrator $\bar{A}(h)$ é contínuo, decrescente e ímpar $(\bar{A}(0)=0)$ enquanto função da sobrevalorização do ativo $h$. Isto significa que o atrator $\bar{A}(h)$ é negativo (positivo), sempre que a sobrevalorização do ativo for positiva (negativa), i.e., sempre que $h>0(h<0)$ (veja a Figura 2.8). Portanto, para uma grande população de agentes, temos que o excesso demanda será positivo (negativo) sempre que a maioria dos agentes financeiros julgarem que o ativo está subvalorizado, i.e., $h<0$ (sobrevalorizado, i.e., $h>0$ ). Neste caso, não ocorrerão bolhas especulativas e nem crashes de preço do ativo. Os conceitos de bolhas especulativas e de crash de preço do ativo serão apresentados abaixo.

Sob baixa heterogeneidade das avaliações individuais sobre o valor fundamental do ativo $\left(\left(\Phi^{\prime}(0)\right)^{-1}<2 \alpha J\right)$, podemos obter um excesso de demanda positivo e estável pelo ativo mesmo que a maioria dos agentes julguem que o ativo esteja sobrevalorizado, mas não excessivamente sobrevalorizado $\left(0<h<h_{*}\right)$. Este é o caso em que bolhas especulativas e crashes de preço do ativo podem ocorrer. Neste caso, i.e., no caso em que $\left(\Phi^{\prime}(0)\right)^{-1}<2 \alpha J$, o sistema de excesso de demanda possui no mínimo um e no máximo dois atratores (excessos de demanda de equilíbrio localmente estáveis). Um deles, denotado por $\bar{A}_{+}(h)$, é positivo; o outro, denotado por $\bar{A}_{-}(h)$, é negativo. Estes atratores possuem as seguintes propriedades enquanto funções da sobrevalorização do ativo $h$ : existe um limiar positivo para a sobrevalorização do ativo dada por $h_{*}>0$ e um excesso de demanda positivo crítico dado por $\bar{A}_{*} \in(0,1)$, tais que:

1. $\bar{A}_{t}$ pertence ao domínio de atração de $\bar{A}_{+}(h)$ sempre que $h \leq h_{*}$ e $\bar{A}_{t}>\bar{A}_{*}$ 
2. $\bar{A}_{t}$ pertence ao domínio de atração do (único) atrator $\bar{A}_{-}(h)$, sempre que $h>h_{*}$

3. Os atratores $\bar{A}_{+}(h)$ e $\bar{A}_{-}(h)$ são decrescentes e contínuos em $h$ em seus respectivos domínios $\left(-\infty, h_{*}\right]$ e $\left[-h_{*}, \infty\right)$, com $1=\bar{A}_{+}(-\infty)>\bar{A}_{+}(h)>0>\bar{A}_{-}(h)>$ $\bar{A}_{-}(\infty)=-1, \forall h \in\left[-h_{*}, h_{*}\right] ; \bar{A}_{-}(-h)=-\bar{A}_{+}(h), \forall h \in\left(-\infty, h_{*}\right]$ (veja a Figura 2.9).

A propriedade 1) implica que o excesso de demanda $\bar{A}_{t}$ pode se manter positivo mesmo quando o ativo estiver sobrevalorizado, mas não "supervalorizado" (excessivamente sobrevalorizado), i.e., quando $0 \leq h<h_{*}$. Este é o caso quando o excesso de demanda $\bar{A}_{t}$ se encontra no domínio de atração do atrator positivo $\bar{A}_{+}(h)$. Definimos uma bolha especulativa positiva (localmente estável) ou simplesmente bolha especulativa o estado do sistema em que o ativo está moderadamente sobrevalorizado, $0<h<h_{*}$, e o excesso de demanda $\bar{A}_{t}$ encontra-se no domínio de atração do atrator positivo $\bar{A}_{+}(h)$ (veja a Figura 2.2: convergência $\bar{A}_{t} \uparrow \bar{A}_{+}(h)$ quando $\left.\bar{A}_{0}>\bar{A}(h)\right)$.

A propriedade 1) implica também que uma mudança da sobrevalorização do ativo de um nível $h$ para um nível $h+\Delta h$, onde $h<h+\Delta h<h_{*}$, leva o sistema a convergir para um novo equilíbrio em $\bar{A}_{+}(h+\Delta h)$ sempre que o sistema no instante $t$, imediatamente anterior a esta intervenção, estiver no equilíbrio $\bar{A}_{+}(h)$ ou próximo ao mesmo, i.e., sempre que $\bar{A}_{t} \geq \bar{A}_{*}$. Isto ocorre pois, segundo a propriedade 1$),\left[\bar{A}_{*}, 1\right]$ pertence ao domínio de atração de todos os atratores $\bar{A}_{+}(h)$, para $h \leq h_{*}$ (veja a Figura 2.6).

Agora, se a nova sobrevalorização do ativo $h+\Delta h$ exceder o limiar $h_{*}$, o excesso de demanda $\bar{A}_{t}$ será atraído da posição $\bar{A}_{+}(h)$ para o único atrator negativo remanescente $\bar{A}_{-}(h+\Delta h)$, cujo domínio de atração é todo o intervalo $[-1,+1]$. Este fato é garantido pela propriedade 2) (veja a Figura 2.7). Note que para reverter um equilíbrio em $\bar{A}_{-}(h+\Delta h)$ para um outro em $\bar{A}_{+}(h)$, onde $-h_{*}<h<h_{*}<h+\Delta h$, não é suficiente eliminar o incremento da sobrevalorização do ativo dado por $\Delta h$. Devido à simetria do sistema, observada com a propriedade 3), uma mudança da sobrevalorização do ativo de $h+\Delta h$ para $h$, onde $-h_{*}<h<h_{*}<h+\Delta h$, levaria o sistema para o equilíbrio $\bar{A}_{-}(h)$ (e não para o equilíbrio $\left.\bar{A}_{+}(h)\right)$. Para levar o sistema de volta ao equilíbrio $\bar{A}_{+}(h)$, a sobrevalorização do ativo tem de ser reduzida a um nível $h^{\prime} \operatorname{com} h^{\prime}<-h_{*}<h$ (i.e., bem abaixo de $h$ ) e depois trazida de volta ao nível $h$. Ou seja, quando a sobrevalorização do ativo ultrapassa o limiar crítico $h_{*}$, o excesso de demanda $\bar{A}_{t}$ não somente cai abruptamente para 


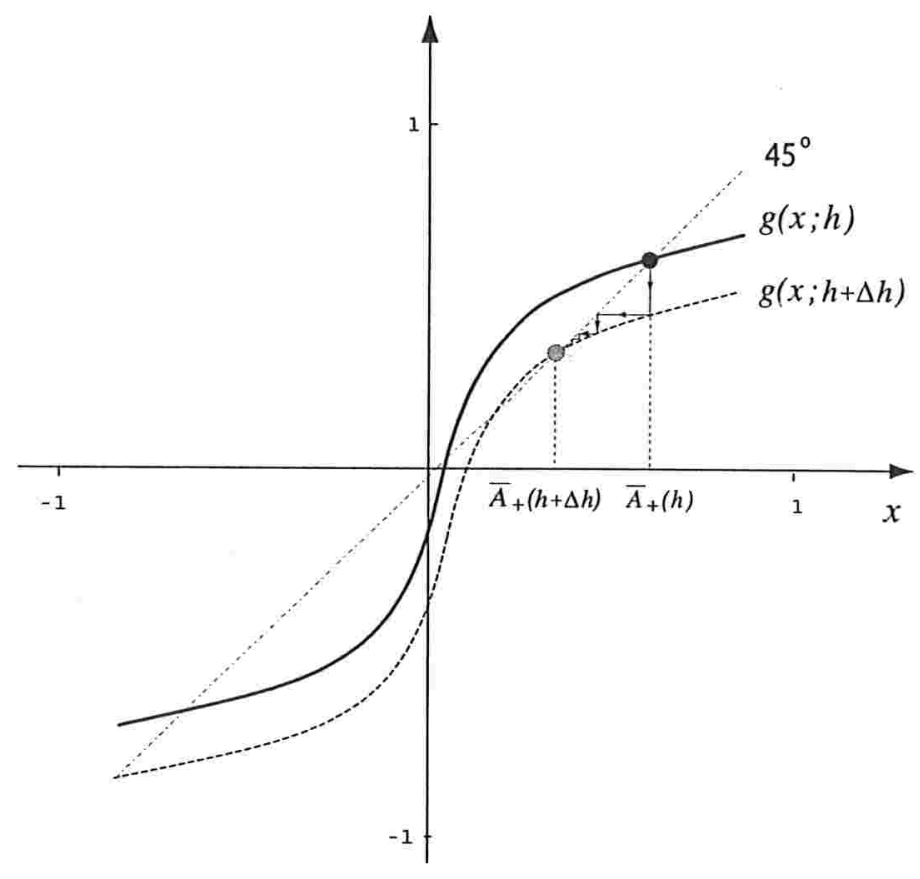

Figura 2.6: Convergência de $\bar{A}_{t}$ para o novo equilíbrio $\bar{A}_{+}(h+\Delta h)$ após uma variação $\Delta h$ moderada da sobrevalorização do ativo $h$, onde $0<h<h+\Delta h<h_{*}$.

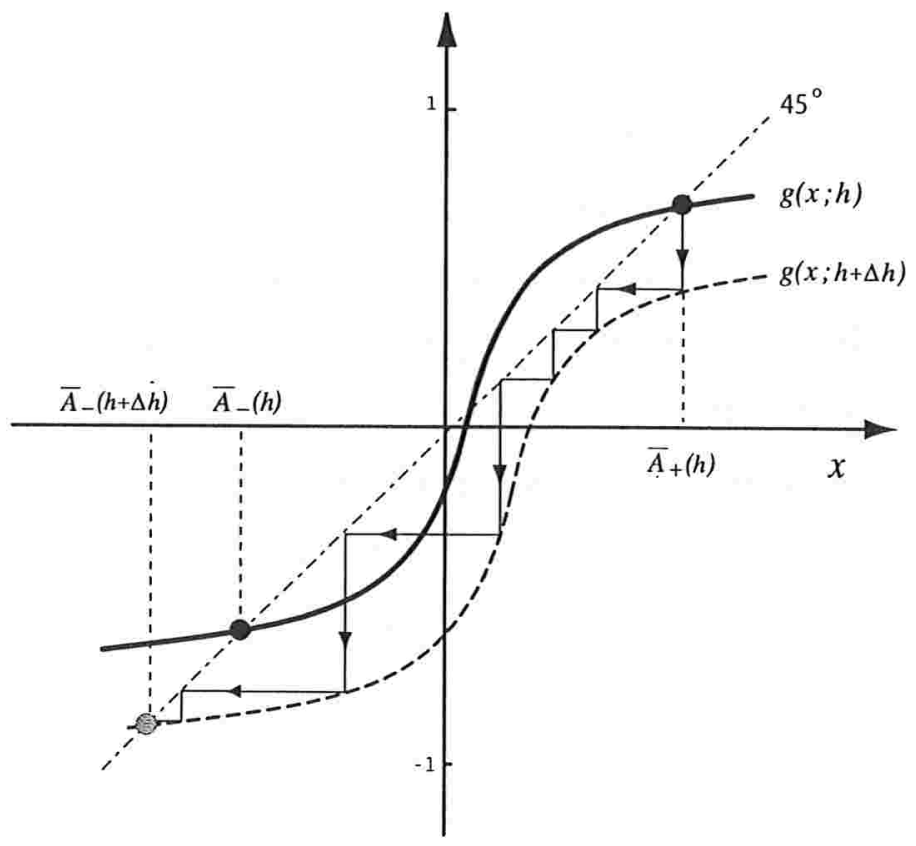

Figura 2.7: Convergência de $\bar{A}_{t}$ para o novo equilíbrio $\bar{A}_{-}(h+\Delta h)$ após uma variação $\Delta h$ da sobrevalorização do ativo $h$ para além da sobrevalrização crítica do ativo, i.e., $0<h<h_{*}<h+\Delta h$. 
um patamar negativo, como também sofre uma mudança de regime enquanto função da sobrevalorização do ativo $h$. Interpretamos esta mudança abrupta de um regime positivo $\bar{A}_{+}(h)$ para um regime negativo de excessos de demanda $\bar{A}_{-}(h)$ enquanto função de $h$ como sendo um crash de preço do ativo resultante de um aumento da sobrevalorização do ativo para além do limiar crítico $h_{*}$. Note que esse resultado é consistente com a intuição socioeconômica: assim que o preço do ativo exceder um limiar acima da avaliação individual média sobre seu valor fundamental $\left(\bar{P}+h_{*}\right)$, ocorre o "estouro" da bolha especulativa seguido do crash de preço do ativo.

Observamos que na vida real uma variação da sobrevalorização do ativo $h\left(h=P_{t}-\bar{P}\right)$ pode ocorrer em função de uma notícia negativa sobre os fundamentos econômicos do ativo, de modo a diminuir a média das avaliações individuais sobre o valor fundamental do ativo dado por $\bar{P}$. Veremos mais adiante que esta sobrevalorização excessiva do ativo implica em uma clara mudança da tendência de preço do ativo quando considerarmos $H_{t}$ variando no tempo $(\lambda>0)$.

De maneira análoga à definição de bolha especulativa positiva e crash de preço do ativo, definimos também uma bolha especulativa negativa e um boom de preço do ativo. Uma bolha especulativa negativa corresponde ao estado do sistema em que o ativo está subvalorizado, mas não excessivamente subvalorizado, $-h_{*}<h<0$, e o excesso de demanda $\bar{A}_{t}$ se encontra no domínio de atração do atrator negativo $\bar{A}_{-}(h)$. Um boom de preço do ativo, ou crash negativo de preço do ativo, ocorre quando $h$ sofre um rebaixamento para um valor menor que $-h_{*}$. Quando esse rebaixamento ocorre, o excesso de demanda $\bar{A}_{t}$ passa a ser atraído para o único atrator positivo $\bar{A}_{+}(h)$. Este é o caso na vida real quando o preço do ativo se torna tão baixo, i.e., abaixo da avaliação individual média sobre o valor fundamental do ativo $\left(P_{t}<\bar{P}-h_{*}\right)$, a ponto de uma quantidade crítica de agentes avaliar que o ativo está demasiadamente subvalorizado e dar, então, início a uma demanda crescente e auto-referenciada pelo ativo.

\subsubsection{Similaridade com o modelo de Ising ferromagnético com interação de longo alcance}

O fenômeno de bolha especulativa seguido de crash de preço do ativo, descrito na seção anterior, é similar ao fenômeno de histerese seguido de transição de fase estudado no modelo de Ising ferromagnético. Pelo nosso modelo, quando a heterogeneidade das avaliações 


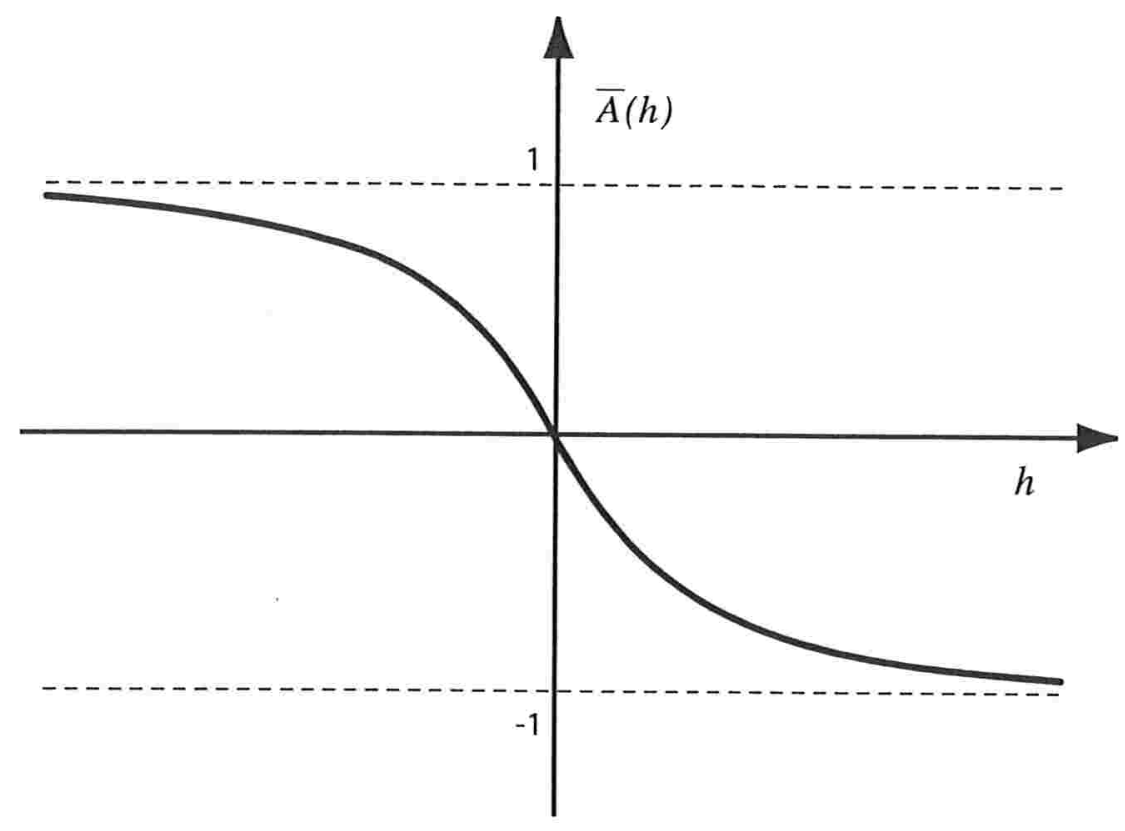

Figura 2.8: Caso $\left(\Phi^{\prime}(0)\right)^{-1} \geq 2 \alpha J$ (alta heterogeneidade das avaliações individuais sobre o valor fundamental do ativo). Gráfico do único equilíbrio globalmente estável de excesso de demanda $\bar{A}(h)$.

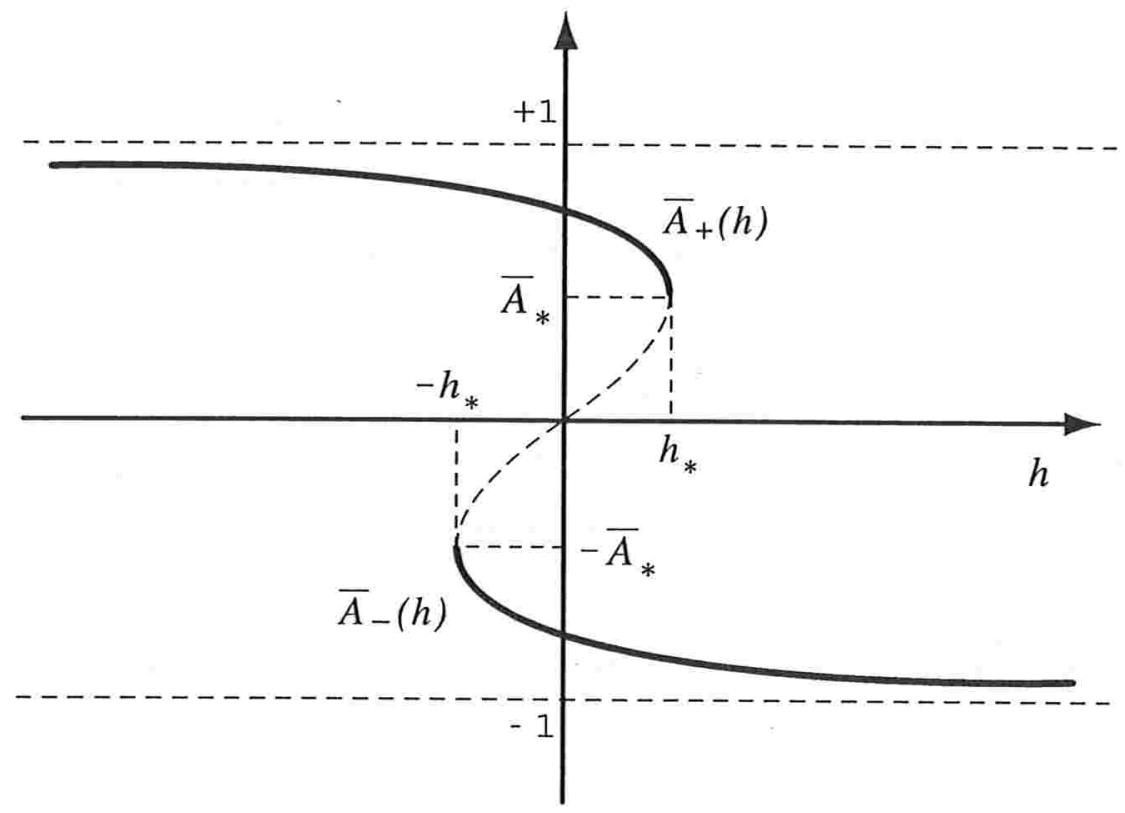

Figura 2.9: Caso $\left(\Phi^{\prime}(0)\right)^{-1}<.2 \alpha J$ (baixa heterogeneidade das avaliações individuais sobre o valor fundamental do ativo). Gráficos dos equilíbrios localmente estáveis de excesso de demanda, $\bar{A}_{-}(h)$ e $\bar{A}_{+}(h)$. 
individuais sobre o valor fundamental do ativo for relativamente baixa em comparação com a influência social de preço $\left(\left(\Phi^{\prime}(0)\right)^{-1}<2 \alpha J\right)$, podemos induzir uma formação de bolha especulativa e consecutivo crash de preço do ativo mediante uma variação da sobrevalorização do ativo $h$ para além do limiar crítico $h_{*}$. De maneira similar, no modelo de Ising, quando a temperatura for relativamente baixa com relação à força de interação entre os spins, podemos induzir o fenômeno de histerese seguido de transição de fase mediante uma variação do campo externo para além de um limiar crítico.

Notamos, no entanto, que não estamos usando o modelo de Ising ferromagnético para descrever os conceitos de bolhas especulativas e crashes de preço do ativo. $\mathrm{Na}$ verdade, a principal contribuição do modelo está na inteligibilidade das relações socioeconômicas modeladas e na generalidade de suas contrapartes matemáticas. Note que a temperatura no modelo de Ising corresponde à quantidade $\left(\Phi^{\prime}(0)\right)^{-1}$, que em nosso modelo mede o quão diferente os agentes avaliam o valor fundamental do ativo. O campo externo do modelo de Ising corresponde, em nosso modelo, à "subvalorização do ativo" $-h$ (valor negativo da sobrevalorização do ativo $h$ ) e a função "tanh" corresponde à função " $2 \Phi-1$ ", onde $\Phi$ é a função de distribuição das avaliações individuais sobre o valor fundamental do ativo (não necessariamente paramétrica). Devido à generalidade de $\Phi$, diferentemente do que ocorre no modelo de Ising, os valores críticos de $h$ para a ocorrência de crash e para ocorrência de boom podem diferir em valor absoluto, desde que a função de densidade de probabilidades $\Phi^{\prime}$ não seja simétrica em torno do zero. Nesta apresentação do nosso modelo, estamos considerando apenas o caso em que $\Phi^{\prime}$ é simétrica em torno do zero. Optamos por nos restringir ao caso simétrico somente para facilitar a exposição dos principais fatos que queremos apresentar. Como conseqüência desta simetria, obtemos que os valores críticos de $h$ para ocorrência de crash e boom são iguais em valor absoluto e são dados por $h_{*}$ e $-h_{*}$, respectivamente.

Um ajuste do modelo a dados reais permite a consideração de funções de densidade de probabilidades não-simétricas em torno do zero, implicando em estimativas diferentes em valor absoluto para as sobrevalorizações críticas de boom e crash de preço do ativo. Observamos que a consideração de tal assimetria não seria possível mediante uma adaptação direta do modelo de Ising aos fenômenos de boom e crash de preço do ativo aqui descritos. 


\subsubsection{Melhoramento da estimativa de convergência do processo estocástico para o sistema dinâmico determinístico}

Lembramos que no começo da Seção 2.4 mostramos a convergência do processo estocástico $\left(W_{t}\right)_{t \geq 0}$ para o sistema dinâmico determinístico $\left(w_{t}\right)_{t \geq 0}$. Aplicamos a Proposição $1 \mathrm{e}$ mostramos que a probabilidade de desvio entre $W_{t}$ e $w_{t}$ tende a zero quando o número de agentes (parâmetro do processo $\left(W_{t}\right)_{t \geq 0}$ ) tende ao infinito. Mais especificamente, estimamos a probabilidade de que $\left\|W_{t}-w_{t}\right\|>\varepsilon$ em função do número de agentes $N$, do tempo $t$ e do desvio $\varepsilon$. Esta estimativa foi dada pela expressão (2.24). Com base nesta convergência, justificamos o estudo do comportamento do processo $W_{t}$ através do estudo de $w_{t}$. Como já observamos na Seção 2.3.1, a estimativa (2.30) apresentada no Corolário 1 da Proposição 1 oferece um maior controle sobre a probabilidade de desvio entre o processo $W_{t}$ e o sistema dinâmico $w_{t}$.

Na presente seção mostraremos que a estimativa (2.24) pode ser substituída pela estimativa (2.30) se garantirmos certas condições adicionais. Em todos os casos analisados neste capítulo, estas condições estarão garantidas desde que $J$ (influência social de preço) ou $K$ (influência social direta) seja suficientemente pequeno ou suficientemente grande em comparação a $\left(\Phi^{\prime}(0)\right)^{-1}$ (heterogeneidade das avaliações individuais sobre o valor fundamental do ativo). Mais precisamente, temos, no presente caso:

Proposição 3. Sejam $\left(W_{t}\right)_{t \geq 0} e\left(w_{t}\right)_{t \geq 0}$ dados como no enunciado da Proposição 1 , onde $Q_{S}$ e $Q_{S}$ são dadas por (2.60) e onde $\lambda=0, K=0$ e $J \geq 0$. Existem constantes $J_{1}$ e $J_{2}$, onde $0<J_{1}<J_{2}$, tais que, se

$$
J<J_{1} \quad \text { ou } \quad J>J_{2}
$$

e $\bar{A}_{0}$ não for um excesso de demanda de equilibrio instável, vale a estimativa (2.30) para a probabilidade em (2.23).

Demonstração. A prova é uma aplicação dos Corolários 1 e 2 da Proposição 1 para $Q_{S}$ e $Q_{F}$ definidas em (2.60) com $K=0$ e $\lambda=0$. Vamos calcular inicialmente $\left\|G^{\prime}(w)\right\|_{\text {max }}$ conforme apresentado no Corolário 2 da Proposição 1 . Sendo $Q_{S}$ e $Q_{F}$ funções constantes em $H$, temos que, para qualquer $w=(H, \bar{S}, \bar{F}) \in \mathbb{R} \times[-1,1]^{2}$, vale $G^{\prime}(w)=(\alpha J+J(1-$ 
$\left.\alpha)+0) 2 \Phi^{\prime}(J \bar{A}-h) ; 0+0+0\right)$, onde $\bar{A}:=\alpha \bar{S}+(1-\alpha) \bar{F}$ e $G(w)$ definido em (2.21). Portanto,

$$
\left\|G^{\prime}(w)\right\|_{\max }=J 2 \Phi^{\prime}(J \bar{A}-h)=\frac{\partial g(\bar{A} ; h)}{\partial \bar{A}}
$$

onde relembramos que $g(x ; h)$ é dada por

$$
g(x ; h):=\alpha[2 \Phi(J x-h)-1]+(1-\alpha)[2 \Phi(-h)-1]
$$

Vamos agora mostrar a validade da hipótese da Proposição 3 no caso $J<J_{1}$ para um dado $J_{1}=J_{1}(\Phi, h, \alpha)$. Mais especificamente, mostraremos a seguir que para

$$
J<J_{1}:=\left(2 \Phi^{\prime}(0)\right)^{-1}
$$

vale a hipótese da Proposição 3.

A prova no caso $J<J_{1}$ é uma aplicação do Corolário 2 da Proposição 1 . Como $\Phi^{\prime}$ assume seu valor máximo em 0 , segue de (2.68) que $\left\|G^{\prime}\right\|_{\max }$ assume seu valor máximo em $w_{*}=(0,0,0)$, onde então

$$
\left\|G^{\prime}\left(w_{*}\right)\right\|_{\max }=J 2 \Phi^{\prime}(0)<1
$$

Observamos que a desigualdade em (2.71) provém de (2.70). Do Corolário 2 da Proposição 1 segue a estimativa (2.30) para a probabilidade (2.24) (probabilidade de $\left\|W_{t}-w_{t}\right\|>\varepsilon$ ).

Vamos agora mostrar a validade da hipótese da Proposição 3 no caso $J>J_{2}$, para um dado $J_{2}=J_{2}(\Phi, h, \alpha)>0$. A seguir, especificaremos a constante $J_{2}=J_{2}(\Phi, h, \alpha)$. Para tanto, observemos inicialmente que, para qualquer $h \in \mathbb{R}$, vale

$$
\lim _{J \rightarrow \infty} \operatorname{Max}\left\{g^{\prime}\left(\bar{A}_{-}(h, J) ; h\right) ; g^{\prime}\left(\bar{A}_{+}(h, J) ; h\right)\right\}=0
$$

onde $g^{\prime}(x ; h)$ denota a derivada da função $g(\cdot ; h): x \mapsto g(x ; h)$. As notações $\bar{A}_{-}(h, J)$ e $\bar{A}_{+}(h, J)$ destacam as dependências entre os pontos fixos $\bar{A}_{-}(h)\left(=\bar{A}_{-}(h, J)\right)$ e $\bar{A}_{+}(h)$ $\left(=\bar{A}_{+}(h, J)\right)$, e o parâmetro $J$ (influência social de preço). ${ }^{7}$

\footnotetext{
${ }^{7} \operatorname{Max}\left\{g^{\prime}\left(\bar{A}_{-}(h, J) ; h\right) ; g^{\prime}\left(\bar{A}_{+}(h, J) ; h\right)\right\}=g^{\prime}\left(\bar{A}_{+}(h, J) ; h\right) \quad\left(g^{\prime}\left(\bar{A}_{-}(h, J)\right)\right.$, quando $\bar{A}_{-}(h, J)$ $\left(\bar{A}_{+}(h, J)\right)$ não estiver definido, i.e., quando $h<-h_{*} \quad\left(h>h_{*}\right)$. Note que para qualquer $h \in \mathbb{R}$, temos $\left(\Phi^{\prime}(0)\right)^{-1}<2 \alpha J$ para $J$ suficientemente grande. Portanto, para $J$ suficientemente grande, existe $h_{*}=h_{*}(J)>0$ e, conseqüentemente, ou $\bar{A}_{-}(h, J)$ ou $\bar{A}_{+}(h, J)$ está definido. Portanto, para $J$ suficientemente grande, $\operatorname{Max}\left\{g^{\prime}\left(\bar{A}_{-}(h, J) ; h\right) ; g^{\prime}\left(\bar{A}_{+}(h, J) ; h\right)\right\}$ está bem definido.
} 
A convergência em (2.72) vem do seguinte fato: como $\Phi$ é uma função de distribuição de probabilidades, a função $g(\cdot ; h)$, que relembramos em $(2.69)$, converge pontualmente (quando $J \rightarrow \infty$ ) para a função constante $x \mapsto \alpha+(1-\alpha)(2 \Phi(-h)-1)$ quando $x>0$ e para a função constante $x \mapsto-\alpha+(1-\alpha)(2 \Phi(-h)-1)$ quando $x<0$. Como $g(\cdot ; h)$ é estritamente crescente e limitada inferiormente e superiormente pelas funções constantes acima mencionadas, podemos facilmente ver que as derivadas de $g(\cdot ; h)$ nos pontos fixos $\bar{A}_{+}(h)$ e $\bar{A}_{-}(h)$ convergem para zero quando $J$ tende ao infinito.

Vamos agora escolher $J_{2}=J_{2}(\Phi, h, \alpha)>0$, tal que

$$
\operatorname{Max}\left\{g^{\prime}\left(\bar{A}_{-}(h, J) ; h\right) ; g^{\prime}\left(\bar{A}_{+}(h, J) ; h\right)\right\}<1 \quad \forall J>J_{2}
$$

Vamos concluir a prova da Proposição 3 no caso em que $J>J_{2}$ para $J_{2}=J_{2}(\Phi, h, \alpha)$ como especificado acima. Para tanto, observemos que, de (2.68) e da continuidade de $g^{\prime}(\cdot ; h)$, segue

$$
\lim _{t \rightarrow \infty}\left\|G^{\prime}\left(w_{t}\right)\right\|_{\max }=\lim _{t \rightarrow \infty} g^{\prime}\left(\bar{A}_{t} ; h\right)=g^{\prime}\left(\lim _{t \rightarrow \infty} \bar{A}_{t} ; h\right)
$$

Agora, se $\bar{A}_{0}$ não é o excesso de demanda de equilíbrio instável, i.e., se $\bar{A}_{0} \neq \bar{A}(h)$, temos que $\bar{A}_{t}$ converge para um dos equilíbrios estáveis $\bar{A}_{-}(h)$ ou $\bar{A}_{+}(h)$ (veja a Figura 2.2). Portanto, qualquer que seja o limite de $\bar{A}_{t}\left(\bar{A}_{-}(h)\right.$ ou $\left.\bar{A}_{+}(h)\right)$, segue de $(2.73)$ a seguinte desigualdade:

$$
\lim _{t \rightarrow \infty}\left\|G^{\prime}\left(w_{t}\right)\right\|_{\max }=g\left(\lim _{t \rightarrow \infty} \bar{A}_{t} ; h\right)<1
$$

Como $\left\|G^{\prime}\left(w_{t}\right)\right\|_{\max } \rightarrow g\left(\lim _{t \rightarrow \infty} \bar{A}_{t} ; h\right)<1$, existem constantes $\gamma>$ e $C>0$, tais que

$$
L\left(w_{t}\right):=\left\|G^{\prime}\left(w_{t}\right)\right\|_{\max }+\gamma<C<1, \quad \forall t>t_{*}
$$

para um dado instante $t_{*}>0$.

Para concluir a Proposição 3, basta observar que as constantes $L\left(w_{t}\right), t=0,1, \ldots$, definidas em (2.76), formam uma seqüência de constantes de Lipschitz no sentido (2.22). Portanto, fazendo $b:=\prod_{\tau=0}^{t_{*}} L\left(w_{\tau}\right)$ e $c:=-\log C(c>0)$, temos

$$
\prod_{\tau=0}^{t} L\left(w_{\tau}\right) \leq b e^{-t c}, \quad \forall t \geq 0
$$

Do Corolário 1 da Proposição 1 segue a estimativa (2.30) para a probabilidade (2.24). 
Observações. A demonstração da Proposição 3 pode ser elucidada e resumida da forma a seguir. Caso $\left(\Phi^{\prime}(0)\right)^{-1} \geq 2 \alpha J$, o excesso de demanda $\bar{A}_{t}$ converge para o (único) equilíbrio globalmente estável $\bar{A}(h)$, onde a derivada de $g(\cdot ; h)$ é tanto menor em valor absoluto quanto menor for $J$. Esta situação é a oposta quando $\left(\Phi^{\prime}(0)\right)^{-1}<2 \alpha J$. Neste caso, o excesso de demanda $\bar{A}_{t}$ converge para um dos equilíbrios $\bar{A}_{-}(h)$ ou $\bar{A}_{+}(h)$, onde a derivada de $g(\cdot ; h)$ é tanto menor em valor absoluto quanto maior for $J$.

Como cada constante de Lipschitz $L\left(w_{t}\right)$ pode ser tomada arbitrariamente próxima à derivada de $g(\cdot ; h)$ em $\bar{A}_{t},(t=0,1, \ldots)$, podemos obter um produtório de constantes de Lipschitz, apropriadamente escolhidas, que convergem exponencialmente rápido para zero se supusermos $\lim _{t \rightarrow \infty} g^{\prime}\left(\bar{A}_{t} ; h\right)<1$. Pela continuidade $g^{\prime}(\cdot ; h)$, basta supor, para tanto, que a derivada de $g(\cdot ; h)$ em $\lim _{t \rightarrow \infty} \bar{A}_{t}$ seja menor que 1 . Para garantir que a derivada de $g(\cdot ; h)$ em $\lim _{t \rightarrow \infty} \bar{A}_{t}$, i.e., nos equilíbrios estáveis de $\bar{A}_{t+1}=g\left(\bar{A}_{t} ; h\right)$, seja menor que 1 , basta supor (pelo exposto no parágrafo anterior) que $J$ seja suficientemente pequeno $\left(J<J_{1}\right)$ ou suficientemente grande $\left(J>J_{2}\right)$. Da convergência exponencial do produtório das contantes de Lipschitz, segue, mediante aplicação do Corolário 1 da Proposição 1 , a estimativa (2.30) para probabilidade (2.23). 


\subsubsection{Caso $J \geq 0, K=0$ e $\lambda>0\left(H_{t}\right.$ varia no tempo)}

Os fenômenos de bolhas especulativas, booms e crashes até agora apresentados dizem respeito a variações da sobrevalorização do ativo $h\left(H_{t}=h\right)$ enquanto parâmetro independente do tempo. Agora analisaremos a evolução do sistema $\left(w_{t}\right)_{t \geq 0}$ quando $H_{t}$ varia no tempo segundo a dinâmica dada em (2.20). Mostraremos como, e sob que condições, os fenômenos de bolhas especulativas, booms e crashes de preço do ativo se auto-organizam. Seguiremos analisando, no entanto, o caso em que $K=0$.

\subsubsection{Dinâmica da sobrevalorização e excesso de demanda do ativo $\left(H_{t}, \bar{A}_{t}\right)_{t \geq 0}$}

Quando $H_{t}$ varia segundo $(2.20)$ e $K=0$, a dinâmica para grandes populações $\left(w_{t}\right)_{t \geq 0}$, onde $w_{t}=\left(H_{t}, \bar{S}_{t}, \bar{F}_{t}\right)(t=0,1, \ldots)$, está unicamente determinada pela dinâmica do par $\left(H_{t}, \bar{A}_{t}\right)$, onde $\bar{A}_{t}=\alpha \bar{F}_{t}+(1-\alpha) \bar{S}_{t}(t=0,1, \ldots)$. Está dinâmica é a seguinte: para $t=0$, temos $\left(H_{0}, \bar{A}_{0}\right)=\left(H_{0}, \alpha \bar{F}_{0}+(1-\alpha) \bar{S}_{0}\right)$, e para $t>0$, segue

$$
\left\{\begin{array}{l}
H_{t}=H_{t-1}+\lambda \bar{A}_{t-1} \\
\bar{A}_{t}=\alpha\left[2 \Phi\left(J \bar{A}_{t-1}-H_{t}\right)-1\right]+(1-\alpha)\left[2 \Phi\left(-H_{t}\right)-1\right]
\end{array}\right.
$$

Denotaremos o sistema dinâmico (2.78) por $\left(H_{t}, \bar{A}_{t}\right)_{t \geq 0}$ e chamaremos este sistema de sistema dinâmico da sobrevalorização e excesso de demanda do ativo.

\subsubsection{Equilíbrios de $\left(H_{t}, \bar{A}_{t}\right)_{t \geq 0}$}

Definiremos agora os equilíbrios de $\left(H_{t}, \bar{A}_{t}\right)_{t \geq 0}$ como segue abaixo. Seja $\digamma: \mathbb{R} \times[-1,1] \rightarrow$ $\mathbb{R} \times[-1,1]$ dada por

$$
\digamma(H, \bar{A}):=(H+\lambda \bar{A}, g(\bar{A} ; H+\lambda \bar{A}))
$$

onde $g$ é definida em (2.62). Definimos $(H, \bar{A}) \in \mathbb{R} \times[-1,1]$ como sendo um equilíbrio de $\left(H_{t}, \bar{A}_{t}\right)_{t \geq 0}$ se valer

$$
(H, \bar{A})=\digamma(H, \bar{A})
$$

Se $(H, \bar{A})$ satisfaz (2.80), diremos também que $(H, \bar{A})$ é um equilíbrio:

1. globalmente estável, quando $\lim _{t \rightarrow \infty} \digamma^{(t)}\left(H_{0}, \bar{A}_{0}\right)=(H, \bar{A}), \quad \forall\left(H_{0}, \bar{A}_{0}\right) \in \mathbb{R} \times[-1,1]$; 
2. localmente estável, quando existir $\varepsilon>0$, tal que: $\lim _{t \rightarrow \infty} F^{(t)}\left(H_{0}, \bar{A}_{0}\right)=(H, \bar{A})$, $\forall\left(H_{0}, \bar{A}_{0}\right) \in B_{\varepsilon}(H, \bar{A}) \cap \mathbb{R} \times[-1,1]$, onde $B_{\varepsilon}(H, \bar{A})$ denota uma bola em $\mathbb{R}^{2}$ de raio $\varepsilon$, centrada em $(H, \bar{A})$;

3. instável, quando $(H, \bar{A})$ não for nem globalmente estável nem localmente estável; onde $\digamma^{(t)}\left(H_{0}, \bar{A}_{0}\right)$ é t-ésima interação de $\digamma$ em $\left(H_{0}, \bar{A}_{0}\right)$. Notamos que, por construção, a dinâmica de $\left(H_{t}, \bar{A}_{t}\right)_{t \geq 0}$ é dada pela interação

$$
\left(H_{t}, \bar{A}_{t}\right)=\digamma^{(t)}\left(H_{0}, \bar{A}_{0}\right), \quad(t=0,1, \ldots)
$$

2.4.2.3 O resultado principal: transição de fase entre existência de um único equilíbrio globalmente estável de $\left(H_{t}, \bar{A}_{t}\right)_{t \geq 0}$ em $(0,0)$ e comportamento cíclico de $\left(H_{t}, \bar{A}_{t}\right)_{t \geq 0}$

Mostraremos agora que o sistema $\left(H_{t}, \bar{A}_{t}\right)_{t \geq 0}$ possui um único equilíbrio globalmente estável em $(0,0)$, i.e., é atraído para $(0,0)$ se a heterogeneidade das avaliações individuais sobre o valor fundamental do ativo for relativamente alta em relação à influência social de preço, i.e., $\left(\Phi^{\prime}(0)\right)^{-1}>2 \alpha J$. Mostraremos também que $\left(H_{t}, \bar{A}_{t}\right)_{t \geq 0}$ é atraído para ciclos correspondendo à auto-organização de bolhas especulativas e crashes de preço do ativo se valer $\left(\Phi^{\prime}(0)\right)^{-1}<2 \alpha J$. Mais especificamente, temos:

Proposição 4. Seja $\left(H_{t}, \bar{A}_{t}\right)$ dado por (2.78). Então, $(0,0)$ é o único equilibrio de $\left(H_{t}, \bar{A}_{t}\right)_{t \geq 0}$. Além disso, existe um número positivo $\lambda_{c}$, tal que, para todo $\lambda$ com $0<$ $\lambda<\lambda_{c}$, vale:

1. Se $\left(\Phi^{\prime}(0)\right)^{-1}>2 \alpha J$

então $(0,0)$ é um equilibrio globalmente estável de $\left(H_{t}, \bar{A}_{t}\right)_{t \geq 0}$;

2. $S e\left(\Phi^{\prime}(0)\right)^{-1}<2 \alpha J$

então $(0,0)$ é um equilibrio instável de $\left(H_{t}, \bar{A}_{t}\right)_{t \geq 0}$ e para $\left(H_{0}, \bar{A}_{0}\right) \neq(0,0)$ vale

(a) $|\mathcal{T}|=\infty$;

(b) $\exists \tau_{\lambda} \in \mathbb{N}:\left|H_{\tau}\right|>h_{*} \forall \tau \in \mathcal{T} \operatorname{com} \tau>\tau_{\lambda}$,

onde $\mathcal{T}=\left\{\tau \in \mathbb{N} \mid \bar{A}_{\tau} \cdot \bar{A}_{\tau+1} \leq 0\right\}$ denota o conjunto dos tempos de reversão de sinal de $\left(\bar{A}_{t}\right)_{t \geq 0}$. 
A prova da Proposição 4 está no apêndice. Daremos agora somente uma idéia intuitiva desta prova.

Idéia intuitiva da prova da Proposição 4. Inicialmente, é claro que $(0,0)$ é um equilíbrio do sistema, pois $\digamma(0,0)=(0,0)$. A idéia para a prova dos resultados restantes faz uso dos resultados da Proposição 2, onde a sobrevalorização do ativo não varia no tempo. Vamos supor inicialmente alta heterogeneidade das avaliações individuais sobre o valor fundamental do ativo, i.e., $\left(\Phi^{\prime}(0)\right)^{-1}>2 \alpha J$. Para $\lambda$ suficientemente pequeno, o sistema $\left(H_{t}, \bar{A}_{t}\right)_{t \geq 0}$ dado por (2.78) está próximo ao sistema auxiliar dado por $\left(H_{t}, \bar{A}\left(H_{t}\right)\right)_{t \geq 0}$, onde $H_{t}$ corresponde à primeira coordenada do sistema $\left(H_{t}, \bar{A}_{t}\right)_{t \geq 0}$ e $\bar{A}\left(H_{t}\right)$ ao único equilíbrio estável do ativo para sobrevalorização fixa do ativo (ao nível $H_{t}$ ), definida no contexto da Proposição 2. Ou seja, $\lim _{\lambda \rightarrow 0, t \rightarrow \infty}\left\|\left(H_{t}, \bar{A}_{t}\right)-\left(H_{t}, \bar{A}\left(H_{t}\right)\right)\right\|=0$. Como $\bar{A}\left(H_{t}\right)$ é decrescente em $H_{t}$ com $\bar{A}(0)=0$, temos que o incremento $H_{t+1}-H_{t}=\lambda \bar{A}_{t}\left(\simeq \lambda \bar{A}\left(H_{t}\right)\right)$ tem o sinal oposto ao de $H_{t}$. Portanto, quando $H_{t}$ é positivo, $H_{t}$ diminui no instante seguinte; quando $H_{t}$ é negativo, $H_{t}$ aumenta no instante seguinte. Por outro lado, pela continuidade de $\bar{A}(\cdot)$ e por $\bar{A}(0)=0$, quanto mais próximo de 0 estiver $H_{t}$, menor será $\bar{A}\left(H_{t}\right)$ em valor absoluto, i.e., menor será o incremento de $H_{t}$ em valor absoluto. Esse mecanismo sugere que $\left(H_{t}, \bar{A}_{t}\right)_{t \geq 0}$ converge para $(0,0)$. Para garantir que $\left(H_{t}, \bar{A}_{t}\right)_{t \geq 0}$ não permaneça oscilando em torno de $(0,0)$, e sim convirja de fato para $(0,0)$, basta supor que $\lambda$ seja suficientemente pequeno.

Vamos supor agora que a heterogeneidade das avaliações individuais sobre o valor fundamental do ativo seja relativamente baixa, i.e., $\left(\Phi^{\prime}(0)\right)^{-1}<2 \alpha J$. Neste caso, a idéia da prova faz uso das propriedades 1), 2), 3) do sistema (2.61) para sobrevalorização fixa do ativo apresentadas na Seção 2.4.1.5. Vamos supor que, no instante inicial, valha

$$
H_{0}<-h_{*} \text { e } \bar{A}_{0} \geq \bar{A}_{*}
$$

Assim sendo, a próxima sobrevalorização do ativo é dada por $H_{1}=H_{0}+\lambda \bar{A}_{0} \geq H_{0}+\lambda \bar{A}_{*}$. Agora, se, para a nova sobrevalorização do ativo ainda valer $H_{1} \leq h_{*}$, a propriedade 1) apresentada na Seção 2.4.1.5 garante que $\bar{A}_{0}$ encontra-se no domínio de atração de $\bar{A}_{+}\left(H_{1}\right)$. Portanto, $\bar{A}_{0}$ é atraído para $\bar{A}_{+}\left(H_{1}\right)$, i.e.,

$$
\left|\bar{A}_{1}-\bar{A}_{+}\left(H_{1}\right)\right| \leq\left|\bar{A}_{0}-\bar{A}_{+}\left(H_{1}\right)\right|
$$


onde $\bar{A}_{+}\left(H_{1}\right)$ é o excesso de demanda de equilíbrio positivo para a sobrevalorização do ativo constante ao nível $H_{1}\left(\leq h_{*}\right)$ apresentada no contexto da Proposição 2.

Agora, se $\bar{A}_{0} \leq \bar{A}_{+}\left(H_{1}\right)$, segue imediatamente de (2.83) $\bar{A}_{0} \leq \bar{A}_{1}$. Como estamos assumindo $\bar{A}_{*} \leq \overline{A_{0}}$, segue $\bar{A}_{*} \leq \overline{A_{1}}$. Se, por outro lado, valer $\bar{A}_{0}>\bar{A}_{+}\left(H_{1}\right)$, segue imediatamente da monotonicidade da função $g\left(\cdot ; H_{1}\right)$

$$
\bar{A}_{1}=g\left(\bar{A}_{0} ; H_{1}\right) \geq g\left(\bar{A}_{+}\left(H_{1}\right) ; H_{1}\right)=\bar{A}_{+}\left(H_{1}\right) \geq \bar{A}_{*}
$$

onde $g$ está definida em (2.62). A primeira igualdade de (2.84) segue imediatamente da definição do sistema dinâmico (2.78); a primeira desigualdade de (2.84) segue do fato de $g\left(\cdot ; H_{1}\right)$ ser uma função monótona crescente; a segunda igualdade de $(2.84)$ segue do fato de $\bar{A}_{+}\left(H_{1}\right)$ ser um ponto fixo de $g\left(\cdot ; H_{1}\right)$; e a última desigualdade de (2.84) segue da suposição $H_{1} \leq h_{*}$ e da propriedade 3) apresentada na Seção 2.4.1.5.

Ou seja, em ambos os casos $\left(\bar{A}_{0} \leq \bar{A}_{+}\left(H_{1}\right)\right.$ ou $\left.\bar{A}_{0}>\bar{A}_{+}\left(H_{1}\right)\right)$ vale $\bar{A}_{1} \geq \bar{A}_{*}$. O mesmo argumento pode ser repetido indefinidamente, de onde concluímos:

$$
\begin{gathered}
\text { iniciando em } H_{0}<-h_{*} \text { e } \bar{A}_{0} \geq \bar{A}_{*} \text {, temos para qualquer } l>0 \text { : } \\
H_{t} \leq h_{*}, \forall t=0,1,2 \ldots l \Rightarrow A_{t} \geq \bar{A}_{*}, \forall t=0,1,2 \ldots l
\end{gathered}
$$

Ocorre que, enquanto $\bar{A}_{t}$ permanecer acima de $\bar{A}_{*}$, os incrementos da sobrevalorização do ativo serão sempre maiores ou iguais a um número positivo, i.e., $H_{t+1}-H_{t} \geq \lambda \bar{A}_{*}>0$. Portanto, iniciando em $H_{0}<-h_{*}$ e $\bar{A}_{0} \geq \bar{A}_{*}$, a sobrevalorização do ativo $H_{t}$ cresce até atingir um valor superior a $h_{*}$. No momento em que $H_{t}>h_{*}$, o excesso de demanda $\bar{A}_{t}$ se encontrará no domínio de atração do único atrator negativo $\bar{A}_{-}\left(H_{t}\right)$. Este fato segue da propriedade 2) comentada na Seção 2.4.1.5. Portanto, assim que $H_{t}>h_{*}$, o excesso de demanda $\bar{A}_{t}$ será atraído para $\bar{A}_{-}\left(H_{t}\right)$, onde, por simetria, vale $\bar{A}_{-}\left(H_{t}\right)<-\bar{A}_{*}$. Como $\lambda$ é relativamente pequeno, podemos mostrar que $\bar{A}_{t}$ decresce monotonamente atingindo valores abaixo de $-\bar{A}_{*}$ enquanto $H_{t}$ se mantém acima de $h_{*}$. Isto é, existirá um momento em que $H_{t}>h_{*}$ e $\bar{A}_{t} \leq-\bar{A}_{*}$. Como essa condição é simétrica à condição inicial (2.82), a dinâmica acima descrita se repete com sinal oposto e $H_{t}$ decresce até atingir um valor inferior a $-h_{*}$. Desta forma é fácil ver que, sob $\left(\Phi^{\prime}(0)\right)^{-1}<2 \alpha J$ e $\lambda$ suficientemente pequeno, qualquer órbita de $\left(H_{t}, \bar{A}_{t}\right)_{t \geq 0}$ é caracterizada por "ciclos" onde $H_{t}$ assume os máximos locais acima de $h_{*}$ e os mínimos locais abaixo de $-h_{*}$. Ou seja, as reversões de 
tendência de preço ocorrem em momentos quando o valor absoluto da sobrevalorização do ativo está acima de $h_{*}$, i.e., $\left|H_{\tau}\right|>h_{*} \forall \tau \in \mathcal{T}$ para $\tau>\tau_{\lambda}$. O número natural $\tau_{\lambda}$ é uma cota superior para o primeiro tempo de reversão $\tau_{*}$ satisfazendo $H_{\tau_{*}}>h_{*}$, i.e., $\tau_{\lambda}>\tau_{*}:=\operatorname{Min}\left\{\tau \in \mathcal{T} \mid H_{\tau}>h_{*}\right\}$.

\subsubsection{Interpretação socioeconômica: estabilidade do preço de equilíbrio de mercado ou formação endógena de bolhas especulativas e crashes de preço}

Interpretaremos agora a dinâmica de $\left(w_{t}\right)_{t \geq 0}$, sob $K=0$ e $\lambda>0$, do ponto de vista socioeconômico.

A Proposição 4 afirma que, quando a heterogeneidade das avaliações individuais sobre o valor fundamental do ativo for suficientemente alta comparada com a influência social de preço $\left(\left(\Phi^{\prime}(0)\right)^{-1} \geq 2 \alpha J\right)$, a sobrevalorização do $H_{t}:=P_{t}-\bar{P}$ e o excesso de demanda $\bar{A}_{t}$ são atraídos para zero. Ou seja, sob alta heterogeneidade das avaliações individuais sobre o valor fundamental do ativo, o preço do ativo converge para a avaliação média $\bar{P}$ e o excesso de demanda $\bar{A}_{t}$, para zero. Ou seja, a avaliação média $\bar{P}$ é um preço de equilíbrio de mercado do ativo, i.e., para este preço, a quantidade de oferta é igual à quantidade de procura pelo ativo (excesso de demanda zero). Este preço de equilíbrio de mercado é globalmente estável (veja a Figura 2.10).

Por outro lado, quando os especuladores são relativamente homogêneos em suas avaliações individuais sobre o valor fundamental do ativo $\left(\left(\Phi^{\prime}(0)\right)^{-1}<2 \alpha J\right)$, bolhas positivas seguidas de crashes de preço do ativo se alternam a bolhas especulativas negativas seguidas de booms de preço do ativo, na medida em que $H_{t}$ passa, sucessiva e alternadamente, da esquerda para a direita e da direita para esquerda pelos limiares $h_{*} \mathrm{e}-h_{*}$, respectivamente. Uma bolha de mercado positiva (negativa) "sobrevive" enquanto o preço de mercado do ativo não ultrapassar o limiar superior de reversão de tendência - limiar de crash - dado por $P_{+}:=\bar{P}+h_{*}$ (não cair abaixo do limiar inferior de reversão de tendência - limiar de boom - dado por $\left.P_{-}:=\bar{P}-h_{*}\right)$ (veja a Figura 2.11 ). 


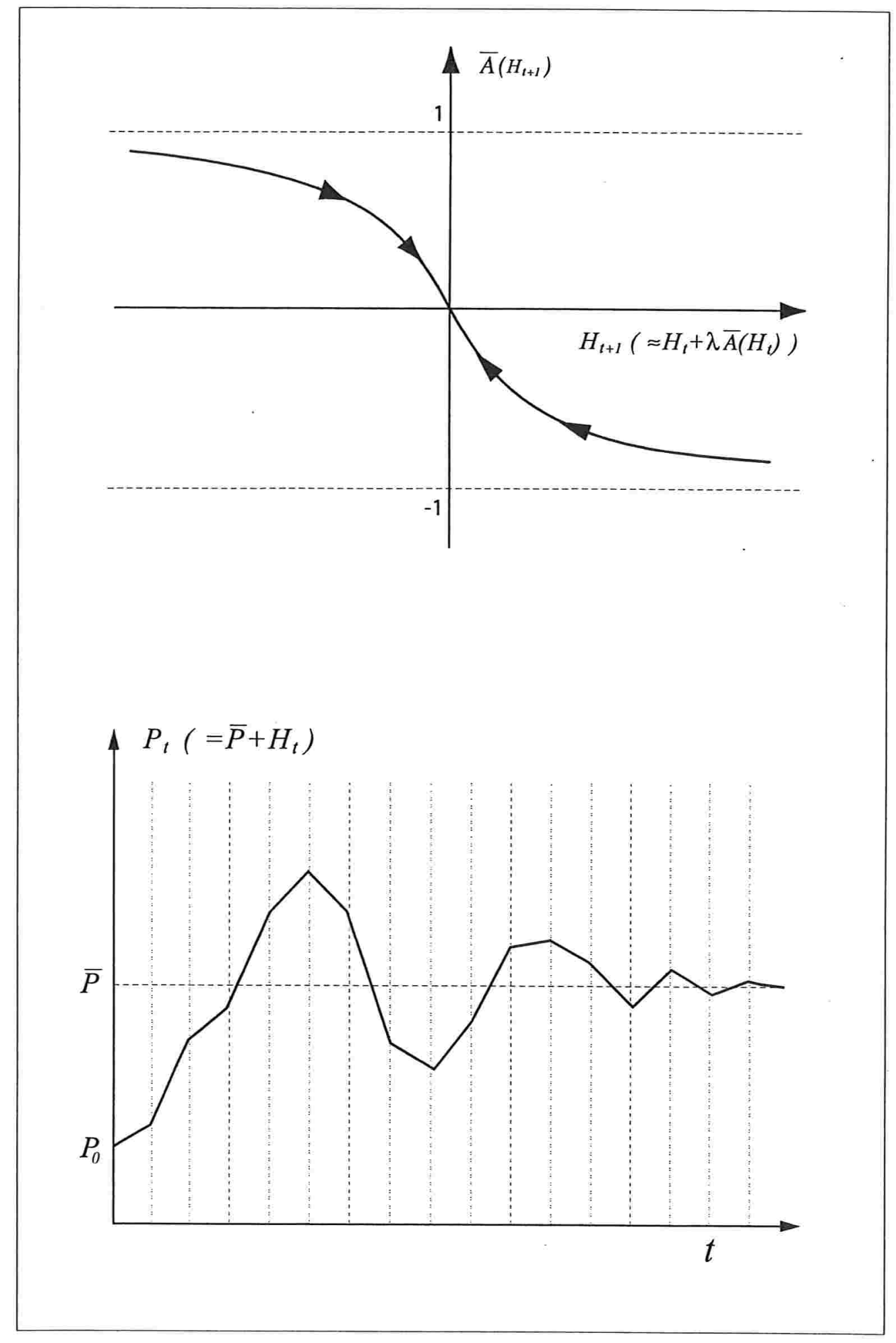

Figura 2.10: Caso $\left(\Phi^{\prime}(0)\right)^{-1}>2 \alpha J$. O primeiro gráfico acima ilustra a convergência de $\left(\bar{A}_{t}, H_{t}\right)$ para $(0,0)$. O segundo gráfico ilustra a correspondente convergência de $P_{t}$ para $\bar{P}$. 


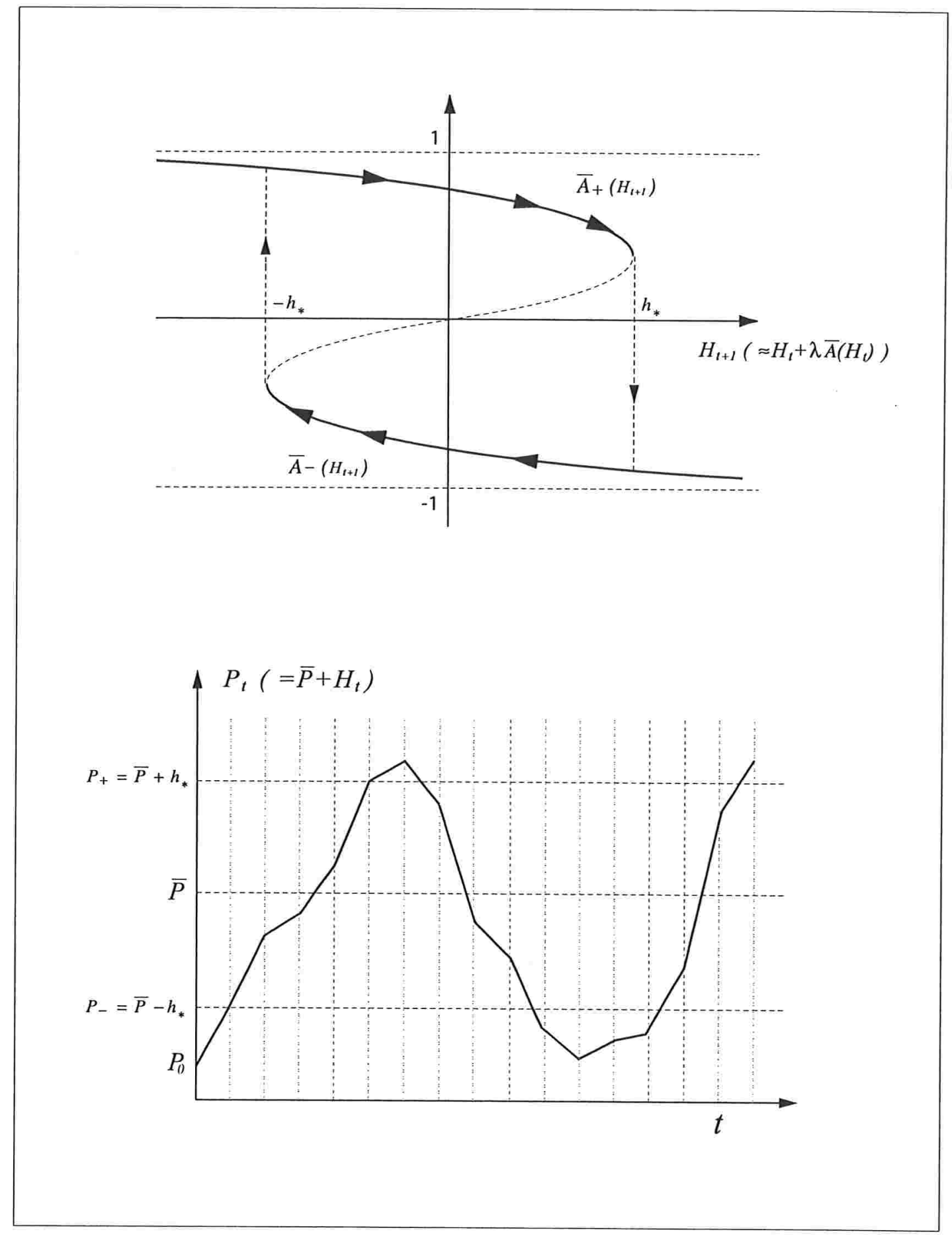

Figura 2.11: Caso $\left(\Phi^{\prime}(0)\right)^{-1}<2 \alpha J$. O primeiro gráfico acima ilustra o comportamento cíclico de $\left(\bar{A}_{t}, H_{t}\right)$. O segundo gráfico ilustra a correspondente dinâmica do preço $P_{t}$. 


\subsubsection{Melhoramento da estimativa de convergência do processo estocástico para o sistema dinâmico determinístico}

Mostraremos agora como a estimativa de convergência de $W_{t}$ para $w_{t}$ pode ser melhorada no caso $K=0$ e $\lambda>0$ mediante aplicação dos Corolários 1 e 2 da Proposição 1. Mais especificamente, temos:

Proposição 5. Sejam $\left(W_{t}\right)_{t \geq 0} e\left(w_{t}\right)_{t \geq 0}$ dados como no enunciado da Proposição 1, onde $Q_{S}$ e $Q_{F}$ são dadas por (2.16) e $\lambda>0, K=0$ e $J \geq 0$. Existem constantes $\lambda_{*}>0$, $\sigma_{*}>0$ e $J_{*}>0$, tais que se $\lambda<\lambda_{*} e$

$$
(\Phi(0))^{-1}>\sigma_{*}(J) \quad \text { ou } \quad J>J_{*}(\Phi, h)
$$

vale a estimativa (2.30) para a probabilidade em (2.23).

Demonstração. A prova é uma aplicação dos Corolários 1 e 2 da Proposição 1 para $Q_{S}$ e $Q_{F}$ definidas em (2.16) com $K=0$ e $\lambda>0$. Vamos calcular inicialmente $\left\|G^{\prime}(w)\right\|_{\max }$ conforme definido no Corolário 2 da Proposição 1. Pela definição de $Q_{S}$ e $Q_{F}$ em (2.16), temos que, para qualquer $w=(H, \bar{S}, \bar{F}) \in \mathbb{R} \times[-1,1]^{2}$, vale $G^{\prime}(w)=(\alpha(J-\lambda)+(1-$ $\left.\alpha)(J-\lambda)-1) 2 \Phi^{\prime}((J-\lambda) \bar{A}-H) ; 0+0-2 \Phi^{\prime}((J-\lambda) \bar{A}-H)\right)$, onde $\bar{A}:=\alpha \bar{S}+(1-\alpha) \bar{F}$ e $G(w)$ definido em (2.21). Portanto,

$$
\left\|G^{\prime}(w)\right\|_{\max }=\operatorname{Max}\{|J-\lambda-1| ; 1\} 2 \Phi^{\prime}((J-\lambda) \bar{A}-H) .
$$

Vamos agora mostrar a validade da hipótese da Proposição 5 no caso $(\Phi(0))^{-1}>\sigma_{*}$ para um dado $\sigma_{*}=\sigma_{*}(J, \lambda)$ (que depende de $J$ e $\lambda$ ). Mais especificamente, mostraremos a seguir que para

$$
(\Phi(0))^{-1}>\sigma_{*}(J, \lambda):=2 \operatorname{Max}\{|J-\lambda-1| ; 1\}
$$

vale a hipótese da Proposição 5.

A prova no caso $(\Phi(0))^{-1}>\sigma_{*}(J, \lambda)$, para $\sigma_{*}(J, \lambda)$ especificado em (2.86), é uma aplicação do Corolário 2 da Proposição 1. Como $\Phi^{\prime}$ assume seu valor máximo em 0, segue de (2.85) que $\left\|G^{\prime}\right\|_{\text {max }}$ assume seu valor máximo em $w_{*}=(0,0,0)$, onde, então,

$$
\left\|G^{\prime}\left(w_{*}\right)\right\|_{\max }=\operatorname{Max}\{|J-\lambda-1| ; 1\} 2 \Phi^{\prime}(0)<1
$$


Observamos que a desigualdade em (2.87) segue de (2.86). Do Corolário 2 da Proposição 1 segue a estimativa (2.30) para a probabilidade (2.24) (probabilidade de $\left\|W_{t}-w_{t}\right\|>\varepsilon$ ).

Vamos agora mostrar a validade da hipótese da Proposição 5 para $J$ suficientemente grande. Daremos apenas uma idéia da prova.

Inicialmente observemos o seguinte: quando $\lambda$ é muito pequeno $\left(\lambda<\lambda_{*}\right)$, o par $\left(H_{t}, \bar{A}_{t}\right)$ na maioria das vezes ou está próximo a $\left(H_{t}, \bar{A}_{+}\left(H_{t}\right)\right)$ ou $\left(H_{t}, \bar{A}_{-}\left(H_{t}\right)\right) \operatorname{com}\left|H_{t}\right|<$ $h_{*}$ (a menos dos momentos de transição "rápida" do regime de excessos de demanda positivo, $\bar{A}_{+}(\cdot)$, para o negativo, $\bar{A}_{-}(\cdot)$, e vice-versa). Lembramos que as notações $\bar{A}_{+}\left(H_{t}\right)$ e $\bar{A}_{-}\left(H_{t}\right)$ designam os equilíbrios de excesso de demanda estáveis positivo e negativo, respectivamente, apresentados no contexto da Proposição 2, i.e., para a sobrevalorização do ativo fixada ao nível $H_{t}$. Como as constantes de Lipschitz de $G$ em $w_{t}$ podem ser tomadas arbitrariamente próximas a $\left\|G^{\prime}\left(w_{t}\right)\right\|_{\max }, t=0,1,2 \ldots$, temos

$$
\begin{aligned}
L\left(w_{t}\right) \simeq\left\|G^{\prime}\left(w_{t}\right)\right\|_{\text {max }} & =\operatorname{Max}\{|J-\lambda-1| ; 1\} 2 \Phi^{\prime}\left((J-\lambda) \overline{A_{t}}-H_{t}\right) \\
& \simeq \operatorname{Max}\{|J-\lambda-1| ; 1\} 2 \Phi^{\prime}\left((J-\lambda) \bar{A}_{ \pm}\left(H_{t}\right)-H_{t}\right)
\end{aligned}
$$

$\operatorname{com} t_{a}^{(i)} \leq t \leq t_{b}^{(i)}, i=0,1,2, \ldots$, para certos $t_{a}^{(i)}, t_{b}^{(i)} \in \mathbb{N}, i=0,1,2, \ldots, \operatorname{com} t_{a}^{(i)} \leq t_{b}^{(i)} \leq$ $t_{a}^{(i+1)}, i=0,1,2, \ldots$ A notação $\bar{A}_{ \pm}\left(H_{t}\right)$ em (2.88) designa o elemento de $\left\{\bar{A}_{-}\left(H_{t}\right), \bar{A}_{+}\left(H_{t}\right)\right\}$ que minimiza o valor absoluto da diferença entre o termo à direita da única igualdade de (2.88) e o termo da segunda linha de (2.88). Observamos que a única igualdade em (2.88) vem de (2.85).

Para $J$ grande e $\lambda$ pequeno, podemos, portanto, aproximar

$$
L\left(w_{t}\right) \simeq J 2 \Phi^{\prime}\left(\left(J \bar{A}_{ \pm}\left(H_{t}\right)-H_{t}\right)=\frac{1}{\alpha} g^{\prime}\left(\bar{A}_{ \pm}\left(H_{t}\right) ; H_{t}\right)\right.
$$

com $t_{a}^{(i)} \leq t \leq t_{b}^{(i)}, i=0,1,2, \ldots$, onde $g^{\prime}\left(\bar{A}_{ \pm}\left(H_{t}\right) ; H_{t}\right)$ denota a derivada de $g\left(\cdot ; H_{t}\right)$ em $\bar{A}_{ \pm}\left(H_{t}\right)$ para $g(\cdot ; h)\left(h=H_{t}\right)$ definida em (2.62). Podemos, portanto, evocar a convergência $\lim _{J \rightarrow \infty} \operatorname{Max}\left\{g^{\prime}\left(\bar{A}_{-}(h, J) ; h\right) ; g^{\prime}\left(\bar{A}_{+}(h, J) ; h\right)\right\}=0$, apresentada em (2.72) na Proposição 3 , e concluir que existe uma constante $C>0$, tal que

$$
L\left(w_{t}\right)<C<1 \quad \text { para } \quad t_{a}^{(i)} \leq t \leq t_{b}^{(i)}, \quad i=0,1,2, \ldots
$$

É fácil ver também que, para $J$ suficientemente grande, podemos escolher as constantes $t_{a}^{(i)}$ e $t_{b}^{(i)}, i=0,1,2 \ldots$, tais que, além de (2.90), valha também

$$
\left\{t_{*}, t_{*}+1, t_{*}+2, \ldots\right\} \subset \cup_{i=1}^{\infty}\left\{t_{a}^{(i)}, t_{a}^{(i)}+1, t_{a}^{(i)}+2, \ldots t_{b}^{(i)}\right\}
$$


para um certo $t_{*}>0$. De (2.90) e (2.91), segue que o produtório $\prod_{\tau=0}^{t} L\left(w_{\tau}\right)$ converge exponencialmente rápido para zero. O resultado segue do Corolário 1 da Proposição 1.

\subsubsection{Caso $J=0, K \geq 0$ e $\lambda=0$ ( $H_{t}=h$ não varia no tempo)}

Vamos analisar nesta seção a dinâmica de $\left(w_{t}\right)_{t \geq 0}$ sob a suposição de que $J=0$, i.e., que os especuladores não sejam influenciados diretamente pela dinâmica do preço (influência social de preço), porém, admitindo $K \geq 0$, i.e., que os especuladores sejam diretamente influenciados pela opinião de outros especuladores (influência social direta - contágio de opinião).

Como na seção anterior, estudaremos inicialmente o sistema dinâmico $\left(w_{t}\right)_{t \geq 0}$ para $\lambda=0$, i.e., $H_{t}=h:=H_{0}$ (fixo) $\forall t \geq 0$. Em seguida, estenderemos esta análise para a $H_{t}$ evoluindo no tempo segundo (2.20) $\operatorname{com} \lambda>0$. As razões para este estudo inicial $(\lambda=0)$ já foram discutidas na Seção 2.4.1.1.

\subsubsection{Dinâmica de excesso de demanda $\left(\bar{A}_{t}\right)_{t \geq 0}$}

Observamos inicialmente que, neste caso particular $\left(J=0, H_{t}=h\right.$ fixo), a dinâmica de $\left(w_{t}\right)_{t \geq 0}$ é unicamente determinada pela dinâmica de $\left(\bar{S}_{t}\right)_{t \geq 0}$. Isto porque, segundo a dinâmica de $\left(w_{t}\right)_{t \geq 0}$ dada em (2.20) e $Q_{S}$ e $Q_{F}$ definidos em (2.60), temos

$$
\bar{S}_{t}=2 \Phi\left(K \bar{S}_{t-1}-h\right)-1 \quad t=1,2, \ldots
$$

onde $H_{t}=h$ e $\bar{F}_{t}=2 \Phi(-h)-1, \forall t \geq 0$. No entanto, focaremos nosso estudo na dinâmica de $\left(\bar{A}_{t}\right)_{t \geq 0}$. Neste caso, de acordo com definição (2.27),

$$
\bar{A}_{t}=\alpha \bar{S}_{t}+(1-\alpha)[2 \Phi(-h)-1] \quad t=1,2, \ldots
$$

onde $\left(S_{t}\right)_{t \geq 0}$ segue a recursão (2.92). A razão pela qual focaremos nosso estudo em $\left(\bar{A}_{t}\right)_{t \geq 0}$ e não em $\left(\bar{S}_{t}\right)_{t \geq 0}$ decorre do fato de $\bar{A}_{t}$ ser diretamente proporcional ao incremento de preço, $P_{t+1}-P_{t}=\lambda \bar{A}_{t}$. Assim sendo, este estudo viabilizará uma melhor compreensão da dinâmica do preço quando supusermos $H_{t}$ variando segundo a dinâmica (2.20) com $\lambda>0$.

A partir das equações (2.92) e (2.93) podemos obter a dependência entre $\bar{A}_{t}$ e $\bar{A}_{t-1}$. Esta dependência é dada por

$$
\bar{A}_{t}=g\left(\bar{A}_{t-1} ; h\right) \quad t=1,2, \ldots
$$


onde $g:[-1,+1] \times \mathbb{R} \rightarrow[-1,+1]$ é dada por

$$
g(x ; h):=\alpha F(K / \alpha \cdot x+(K(1-\alpha) / \alpha) F(h)-h)+(1-\alpha) F(-h)
$$

onde $F:=2 \Phi-1$.

\subsubsection{Excesso de demanda de equilíbrio}

Nosso objetivo agora é estudar os equilíbrios do sistema de excessos de demanda dado por (2.94) para diferentes regiões dos parâmetros $K,\left(\Phi^{\prime}(0)\right)^{-1}$, $\alpha$ e $h$. Estes equilíbrios são soluções em $\bar{A} \in[-1,1]$ da equação

$$
\bar{A}=g(\bar{A} ; h)
$$

que serão diferenciados em equilíbrios globalmente estáveis, localmente estáveis e equilíbrios instáveis. Esta diferenciação será feita da mesma forma como diferenciamos os equilíbrios do sistema $\left(\bar{A}_{t}\right)_{t \geq 0}$, dado em (2.61), em equilíbrios globalmente estáveis, localmente estáveis e instáveis (veja a Seção 2.4.1.3).

Observamos que esta análise não será feita mediante a forma explícita de $g$ dada em (2.95). Para facilitar este estudo, faremos uso dos equilíbrios $\left(\bar{A}_{t}\right)_{t \geq 0}$ dados em (2.61), no caso em que $\alpha=1$. Os resultados obtidos neste caso facilitam a análise do presente caso no seguinte sentido: como a dinâmica de $\left(\bar{A}_{t}\right)_{t \geq 0}$ dada em (2.93) é função linear da dinâmica de $\left(\bar{S}_{t}\right)_{t \geq 0}$, onde $\bar{S}_{t}=2 \Phi\left(K \bar{S}_{t-1}-h\right)-1, t=1,2, \ldots$, segue que qualquer equilíbrio $\bar{A}$ de $\left(\bar{A}_{t}\right)_{t \geq 0}$ satisfaz $\bar{A}=\alpha \bar{S}+(1-\alpha)[2 \Phi(-h)-1]$, onde $\bar{S}$ satisfaz a condição de equilíbrio para o sistema $\left(\bar{S}_{t}\right)_{t \geq 0}$, i.e., $\bar{S}=2 \Phi(K \bar{S}-h)-1$. Ocorre que a dinâmica de $\bar{S}_{t}=2 \Phi\left(K \bar{S}_{t-1}-h\right)-1, t=1,2, \ldots$, é um caso particular da dinâmica de $\left(\bar{A}_{t}\right)_{t \geq 0}$ dada em (2.61) quando $\alpha=1$ e $J=K$. Logo, como a dinâmica de $\left(\bar{A}_{t}\right)_{t \geq 0}$ dada em (2.61) já foi estudada (em particular para $\alpha=1$ e $J=K$ ), podemos estudar o comportamento de $\left(\bar{A}_{t}\right)_{t \geq 0}$ dada em (2.94) (lado esquerdo de (2.96)) através do estudo da seqüência expressa do lado direito da seguinte identidade:

$$
\bar{A}_{t}=\alpha \bar{A}_{t}^{(1)}+(1-\alpha)[2 \Phi(-h)-1]
$$

onde $\left(\bar{A}_{t}^{(1)}\right)_{t \geq 0}$ denota a seqüência definida em (2.61) para $\alpha=1$ e $J=K$. 


\subsubsection{Transição de fase entre excessos de demanda de equilíbrio}

A igualdade apresentada em (2.96) nos permite identificar e classificar mais facilmente os equilíbrios de (2.94) com base nos equilíbrios de $\left(\bar{A}_{t}^{(1)}\right)_{t \geq 0}$ já identificados e classificados mediante a Proposição 2. Posto isto, seguem abaixo as conclusões para os equilíbrios de (2.94).

1. Se $\left(\Phi^{\prime}(0)\right)^{-1} \geq 2 K$ ou $\alpha=0$

então $\bar{A}(h):=\alpha \bar{A}^{(1)}(h)+(1-\alpha)(2 \Phi(-h)-1)$ é o único equilíbrio globalmente estável de $(2.94) ; \bar{A}(h)$ é contínuo, ímpar e decrescente como função de $h \in \mathbb{R}$.

2. Se $\left(\Phi^{\prime}(0)\right)^{-1}<2 K$ e $\alpha \in(0,1]$

então o sistema (2.94) tem no mínimo um e no máximo dois equilíbrios localmente estáveis, denotados por $\bar{A}_{+}(h)$ e $\bar{A}_{-}(h)$. Estes equilíbrios têm as seguintes propriedades enquanto funções de $h$ :

(a) Se $h \in\left(-\infty, h_{*}^{(1)}\right]$

então $\bar{A}_{+}(h):=\alpha \bar{A}_{+}^{(1)}(h)+(1-\alpha)(2 \Phi(-h)-1)$

(b) Se $h \in\left[-h_{*}^{(1)},+\infty\right)$

então $\bar{A}_{-}(h):=\alpha \bar{A}_{-}^{(1)}(h)+(1-\alpha)(2 \Phi(-h)-1)$

(c) $\bar{A}_{+}(h)$ e $\bar{A}_{-}(h)$ são decrescentes e contínuos em seus respectivos domínios de definição, $\left(-\infty, h_{*}^{(1)}\right]$ e $\left[-h_{*}^{(1)}, \infty\right)$, com $1=\bar{A}_{+}(-\infty)>\bar{A}_{+}(h)>\bar{A}_{-}(h)>$ $\bar{A}_{-}(\infty)=-1, \forall h \in\left[-h_{*}^{(1)}, h_{*}^{(1)}\right]$,

onde $\bar{A}_{+}^{(1)}(h)$ e $\bar{A}_{-}^{(1)}(h)$ denotam os equilíbrios localmente estáveis de excesso de demanda positivo e negativo do sistema (2.61) apresentados no contexto da Proposição 2 quando $J=K$ e $\alpha=1$. A quantidade $h_{*}^{(1)}$ denota a sobrevalorização crítica positiva do ativo definida no contexto da Proposição 2 quando $J=K$ e $\alpha=1$, i.e., $h_{*}^{(1)}$ é unicamente determinado por

$$
\left\{\begin{array}{l}
h_{*}^{(1)}>0 \\
\bar{A}_{*}^{(1)}=2 \Phi\left(K \bar{A}_{*}^{(1)}-h_{*}^{(1)}\right)-1 \\
1=2 \Phi^{\prime}\left(K \bar{A}_{*}^{(1)}-h_{*}^{(1)}\right) K
\end{array}\right.
$$




\subsubsection{O resultado principal: negatividade do atrator superior}

Como veremos a seguir, no presente caso, $J=0, K \geq 0$ e $\lambda=0$, a positividade do equilíbrio localmente estável $\bar{A}_{+}(h)$ (negatividade de $\left.\bar{A}_{-}(h)\right)$ não está garantida. Chamaremos agora, portanto, o equilíbrio $\bar{A}_{+}(h)\left(\bar{A}_{-}(h)\right)$ de "atrator superior" ("atrator inferior").

Daremos agora a condição necessária e suficiente para que o atrator superior $\bar{A}_{+}(h)$ assuma valores negativos e o atrator inferior $\bar{A}_{-}(h)$, valores positivos. Para que os atratores superior e inferior existam, estaremos supondo $\Phi^{\prime}(0)^{-1}<2 K$ e $0<\alpha<1$. Devido à continuidade e à monotonicidade de $\bar{A}_{-}(h)$ e $\bar{A}_{+}(h)$ mencionadas em (c), o atrator superior $\bar{A}_{+}(h)$ (inferior $\bar{A}_{-}(h)$ ) assume valores negativos (positivos) se, e somente se, $\bar{A}_{+}\left(h_{*}^{(1)}\right)<0<\bar{A}_{-}\left(-h_{*}^{(1)}\right)$. Devido à simetria do sistema, as duas últimas desigualdades são equivalentes à seguinte condição:

$$
\bar{A}_{+}\left(h_{*}^{(1)}\right)=\alpha \bar{A}_{+}^{(1)}\left(h_{*}^{(1)}\right)+(1-\alpha)\left(2 \Phi\left(-h_{*}^{(1)}\right)-1\right)<0
$$

Observamos que $\bar{A}_{+}^{(1)}\left(h_{*}^{(1)}\right)$ é igual a $\bar{A}_{*}^{(1)}$, isto é, que $\bar{A}_{+}^{(1)}\left(h_{*}^{(1)}\right)$ corresponde ao excesso de demanda crítico positivo e $h_{*}^{(1)}$ à sobrevalorização crítica positiva do ativo dados no contexto da Proposição 2 para $\alpha=1$ e $J=K$. Agora, como $h_{*}^{(1)}>0$ e, de acordo com as propriedades de $\Phi$ dadas em (2.5), a função $2 \Phi$ - 1 é uma função ímpar e crescente, vale $2 \Phi\left(-h_{*}^{(1)}\right)-1<0$. Portanto, a condição (2.98) estará garantida se, e somente se, $\alpha$ for suficientemente pequeno, isto é, se, e somente se,

$$
\alpha<\alpha_{*}:=\frac{\left[2 \Phi\left(h_{*}^{(1)}\right)-1\right]}{\bar{A}_{*}^{(1)}+\left[2 \Phi\left(h_{*}^{(1)}\right)-1\right]} \quad(<1)
$$

onde $h_{*}^{(1)}$ e $\bar{A}_{*}^{(1)}$ são dados por (2.97).

Portanto, quando $\Phi^{\prime}(0)^{-1}<2 K$ e $0<\alpha<\alpha_{*}$, temos a seguinte situação: $\bar{A}_{+}(h)$ assume valores positivos quando $h<\left[\bar{A}_{+}\right]^{-1}(0)$, é igual a zero quando $h=\left[\bar{A}_{+}\right]^{-1}(0)$ e assume valores negativos quando $\left[\bar{A}_{+}\right]^{-1}(0)<h \leq h_{*}^{(1)}$. Propriedades análogas valem para o atrator inferior $\bar{A}_{-}(h)$ enquanto função de $h$ (veja a Figura 2.12).

A negatividade do atrator superior e a positividade do atrator inferior discutidas acima e ilustradas na Figura 2.12 jogam um papel crucial na ocorrência de dois equilíbrios localmente estáveis do sistema dinâmico da sobrevalorização e excesso de demanda do ativo $\left(H_{t}, \bar{A}_{t}\right)_{t \geq 0}$ para $\lambda>0$, i.e., ao considerarmos $H_{t}$ variando no tempo. Heuristicamente, 


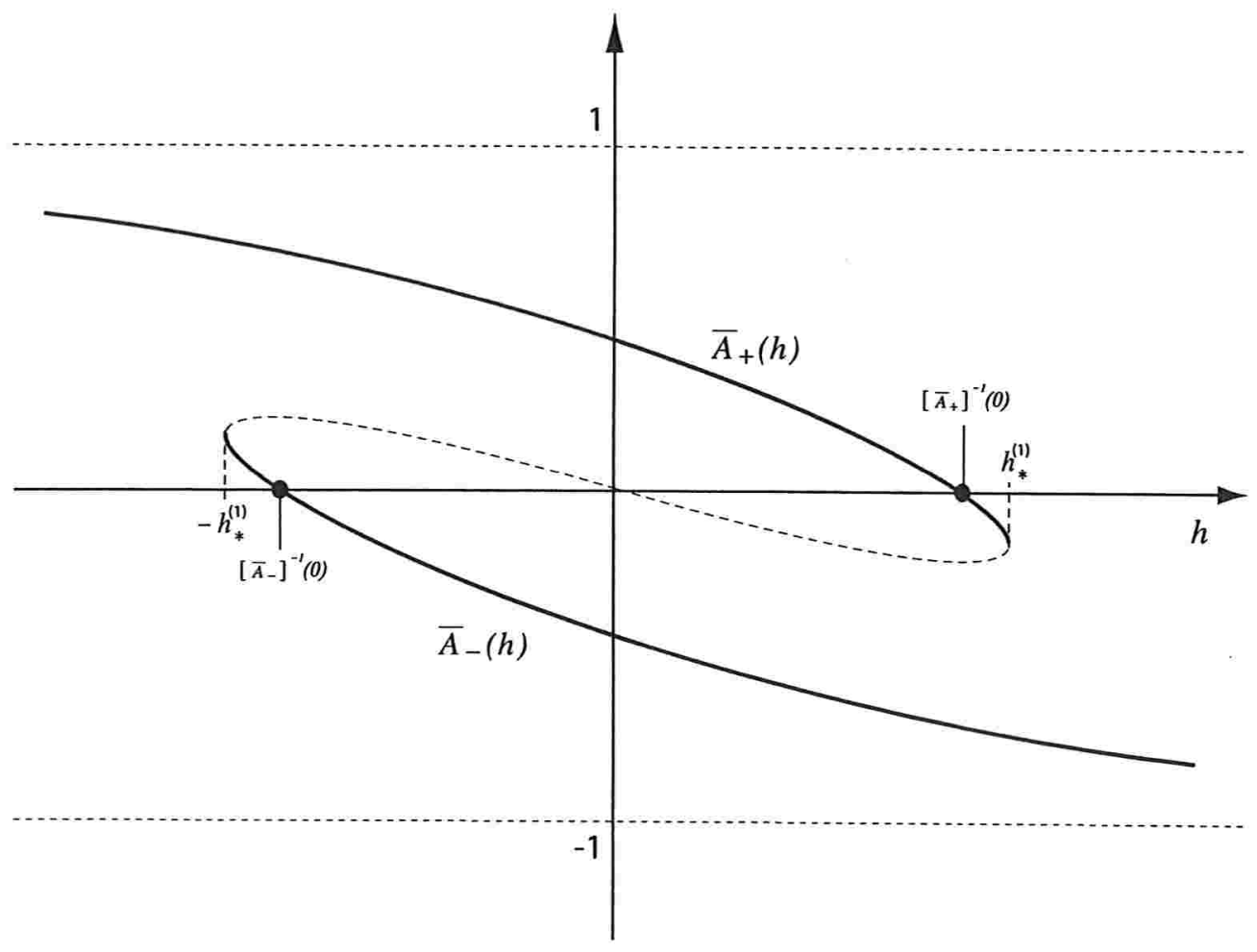

Figura 2.12: Caso $\left(\Phi^{\prime}(0)\right)^{-1}<2 K$ e $0<\alpha<\alpha^{*}$. Gráficos de $\bar{A}_{+}(h)$ e $\bar{A}_{-}(h)$.

estes equilíbrios localmente estáveis já podem ser identificados. Eles são $\left(\left[\bar{A}_{-}\right]^{-1}(0), 0\right)$ e $\left(\left[\bar{A}_{+}\right]^{-1}(0), 0\right)$, onde $\left[\bar{A}_{-}\right]^{-1}(0)<0<\left[\bar{A}_{+}\right]^{-1}(0)$.

Melhoramento da estimativa da probabilidade de desvio entre $W_{t}$ e $w_{t}$. Observamos que a estimativa da probabilidade de desvio entre $W_{t}$ e $w_{t}$, onde $J=0$ e $\lambda=0$, pode ser melhorada mediante a aplicação dos Corolários 1 e 2 da Proposição 1 . Isto é, a cota superior (2.24) para a probabilidade em (2.23), onde $J=0$ e $\lambda=0$, pode ser substituída por uma estimativa do tipo (2.30), se supusermos $K\left\langle K_{1}\right.$ ou $K>K_{2}$ para $K_{1}$ suficientemente pequeno e $K_{2}$ suficientemente grande. Nesta seção omitiremos o tratamento desta questão. Observamos apenas que o sentido e a prova desta afirmação são análogos aos correspondentes da seção anterior na qual tratamos essa questão para $K=0$ e $\lambda=0$. 


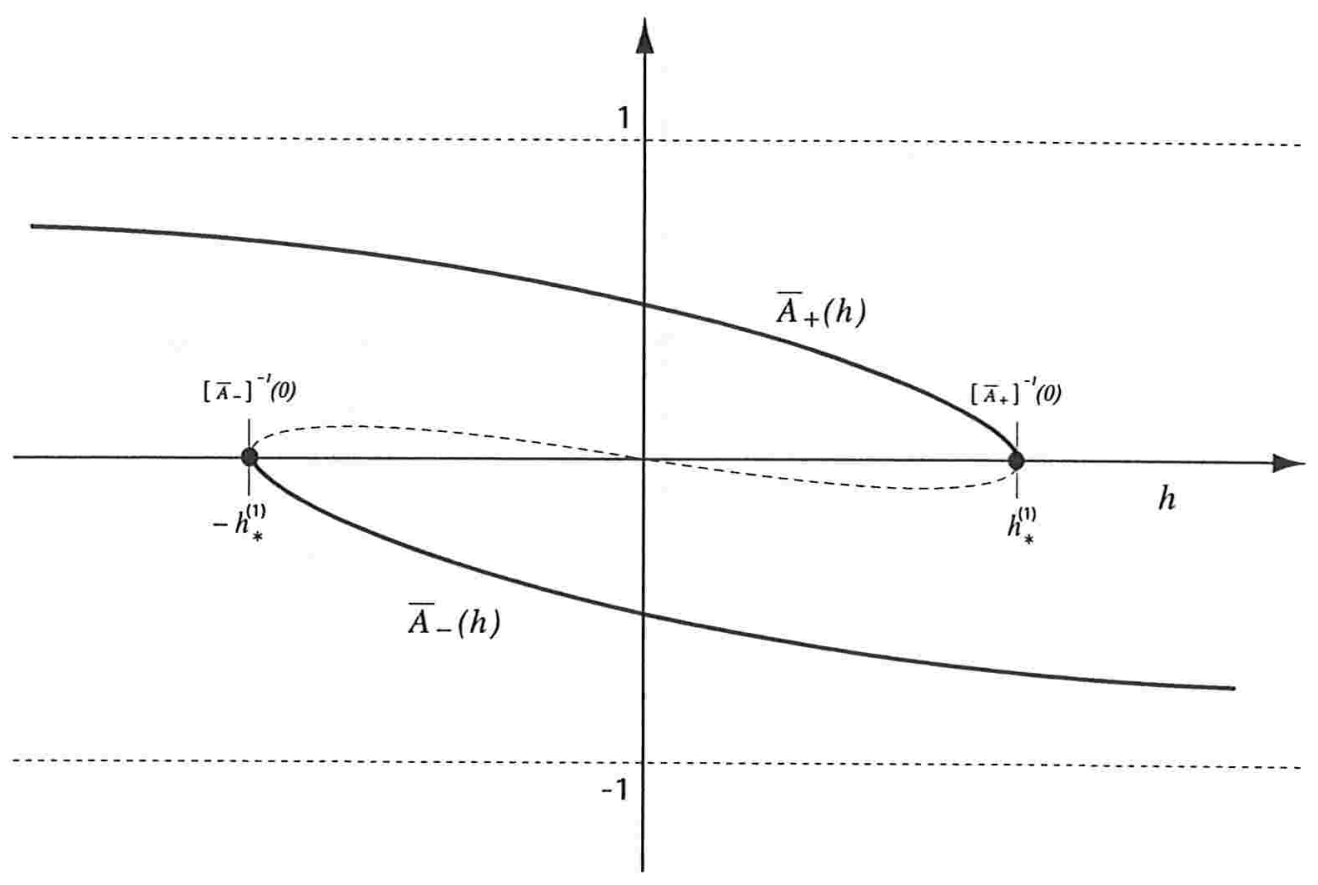

Figura 2.13: Caso $\left(\Phi^{\prime}(0)\right)^{-1}<2 K$ e $\alpha=\alpha^{*}$. Gráficos de $\bar{A}_{+}(h)$ e $\bar{A}_{-}(h)$.

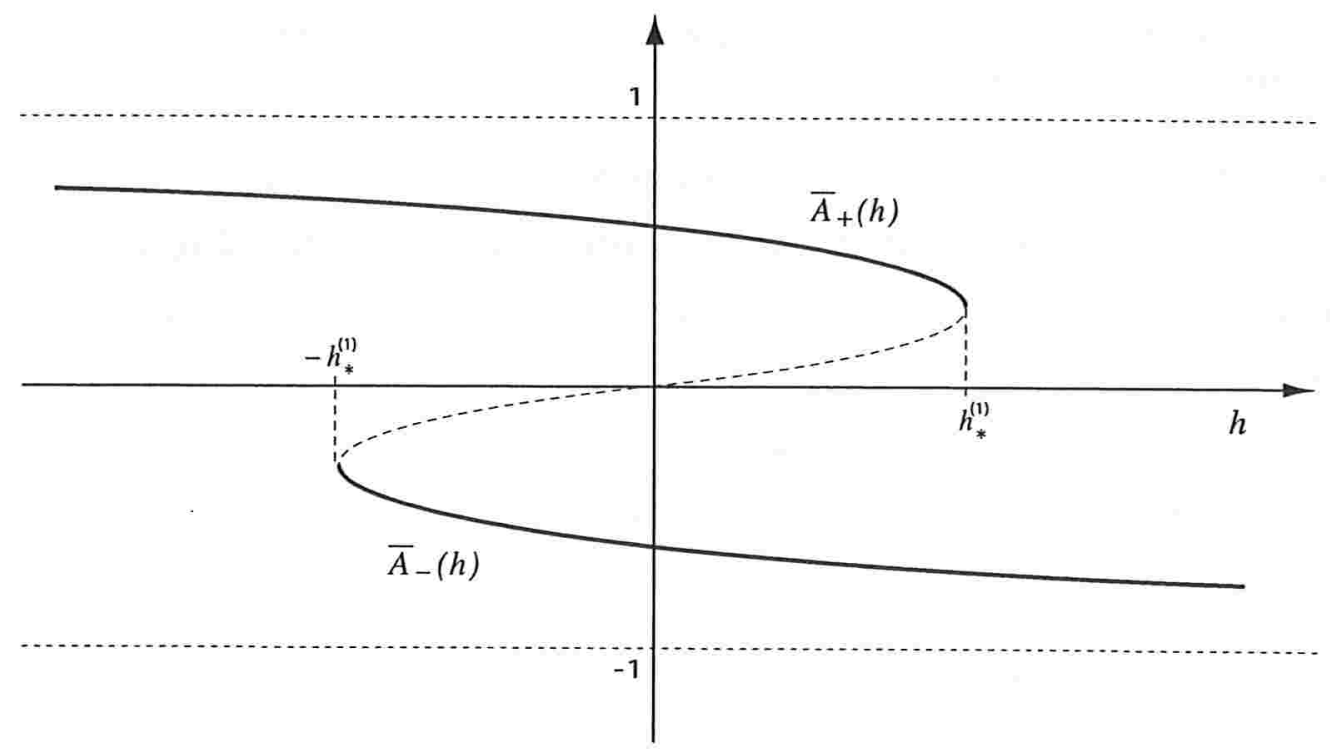

Figura 2.14: Caso $\left(\Phi^{\prime}(0)\right)^{-1}<2 K$ e $\alpha>\alpha^{*}$. Gráficos de $\bar{A}_{+}(h)$ e $\bar{A}_{-}(h)$. 


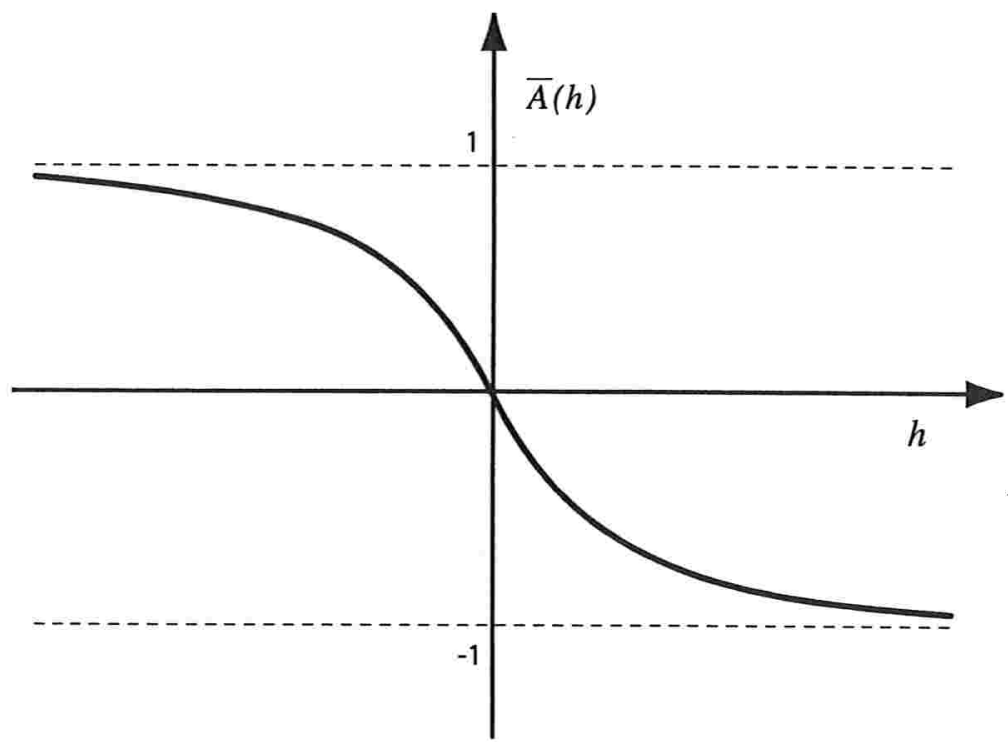

Figura 2.15: Caso $\left(\Phi^{\prime}(0)\right)^{-1} \geq 2 K$ ou $\alpha=0$. Gráfico de $\bar{A}(h)$.

\subsubsection{Caso $J=0, K \geq 0$ e $\lambda>0$ ( $H_{t}$ varia no tempo)}

Estudaremos agora o comportamento do sistema $\left(w_{t}\right)_{t \geq 0}$ para o caso em que $J=0, K \geq 0$ e $\lambda>0$.

\subsubsection{Dinâmica da sobrevalorização e excesso de demanda do ativo $\left(H_{t}, \bar{A}_{t}\right)_{t \geq 0}$}

Neste caso, a dinâmica para grandes populações $\left(w_{t}\right)_{t \geq 0}$, onde $w_{t}=\left(H_{t}, \bar{S}_{t}, \bar{F}_{t}\right)(t=$ $0,1, \ldots)$, está unicamente determinada pelo sistema dinâmico da sobrevalorização e excesso de demanda do ativo $\left(H_{t}, \bar{A}_{t}\right)(t=0,1, \ldots)$. Esta dinâmica é dada pela seguinte interação:

$$
\left\{\begin{array}{l}
H_{t}=H_{t-1}+\lambda \bar{A}_{t-1} \\
\bar{A}_{t}=g\left(\bar{A}_{t-1} ; H_{t-1}+\lambda \bar{A}_{t-1}\right)
\end{array} \quad t=1,2,3 \ldots\right.
$$

onde $g$ é dada por (2.95).

Nosso objetivo agora é estudar os equilíbrios do sistema de sobrevalorização e excesso de demanda do ativo dado por (2.100) para diferentes regiões dos parâmetros $K,\left(\Phi^{\prime}(0)\right)^{-1}$ e $\alpha$. Estes equilíbrios satisfazem $(h, \bar{A})=\digamma(h, \bar{A})$, onde $\digamma: \mathbb{R} \times[-1,1] \rightarrow \mathbb{R} \times[-1,1]$ com $\digamma(h, \bar{A})=(h+\lambda \bar{A}, g(\bar{A} ; h+\lambda \bar{A}))$, com $g$ definida em (2.95) nesta seção. Os equilíbrios de (2.100) serão diferenciados em equilíbrios globalmente estáveis, localmente estáveis e 
instáveis, da mesma forma como os equilíbrios do sistema $\left(H_{t}, \bar{A}_{t}\right)_{t \geq 0}$, dados pela interação (2.81), foram classificados nestas categorias na Seção 2.4.2.2.

\subsubsection{O resultado principal: transição de fase entre existência de dois equilíbrios estáveis de $\left(H_{t}, \bar{A}_{t}\right)_{t \geq 0}$ e comportamento cíclico de $\left(H_{t}, \bar{A}_{t}\right)_{t \geq 0}$}

Mostraremos agora as propriedades do sistema dinâmico da sobrevalorização e excesso de demanda do ativo $\left(H_{t}, \bar{A}_{t}\right)_{t \geq 0}$. Sob alta heterogeneidade das avaliações individuais sobre o valor fundamental do ativo, o sistema $\left(H_{t}, \bar{A}_{t}\right)_{t \geq 0}$ é atraído para $(0,0)$, i.e., $\left(H_{t}, \bar{A}_{t}\right)_{t \geq 0}$ possui um único equilíbrio globalmente estável em $(0,0)$. Sob baixa heterogeneidade das avaliações individuais sobre o valor fundamental do ativo, $(0,0)$ também é um equilíbrio do sistema, porém este equilíbrio é instável; se, além disso, i.e., se, além de a heterogeneidade das avaliações individuais sobre o valor fundamental do ativo ser relativamente baixa, a fração de especuladores for positiva, porém, suficientemente pequena, então dois outros equilíbrios localmente estáveis surgirão. Se, no entanto, a fração de especuladores for suficientemente grande, o sistema $\left(H_{t}, \bar{A}_{t}\right)_{t \geq 0}$ será atraído para um "ciclo" que corresponde à auto-organização de bolhas especulativas e crashes de preço do ativo no sentido já descrito na seção anterior. Mais especificamente, temos:

Proposição 6. Seja $\left(H_{t}, \bar{A}_{t}\right)_{t \geq 0}$ dado por (2.100). Inicialmente, temos que $(0,0)$ é sempre um equilibrio de $\left(H_{t}, \bar{A}_{t}\right)_{t \geq 0}$. Além disso, existe um número positivo $\lambda_{c}$, tal que, para todo $\lambda \operatorname{com} 0<\lambda<\lambda_{c}$, vale:

\section{Se $\left(\Phi^{\prime}(0)\right)^{-1}>2 K$ ou $\alpha=0$}

então $(0,0)$ é o único equilíbrio globalmente estável de $\left(H_{t}, \bar{A}_{t}\right)_{t \geq 0}$.

2. Se $\left(\Phi^{\prime}(0)\right)^{-1}<2 K$

então $(0,0)$ é um equilíbrio instável de $\left(H_{t}, \bar{A}_{t}\right)_{t \geq 0}$. Se, além de $\left(\Phi^{\prime}(0)\right)^{-1}<2 K$ valer para $\alpha_{*}$ dado em (2.99):

(a) $0<\alpha \leq \alpha_{*}$

então $\left(H_{t}, \bar{A}_{t}\right)$ possui dois outros equilibrios, denotados por $E_{+} e E_{-}$, onde $E_{-}=-E_{+} \operatorname{com} E_{+}:=\left(\left[\bar{A}_{+}\right]^{-1}(0), 0\right) ; 0<\left[\bar{A}_{+}\right]^{-1}(0)<h_{*}^{(1)}$ e $h_{*}^{(1)}$ é unicamente determinado por (2.97). Para estes equilibrios, vale: 
i. Se $0<\alpha<\alpha^{*}$

então $E_{+}$e $E_{-}$são localmente estáveis, onde

$\left\{(h, 0) \mid h<-h_{*}^{(1)}\right.$ ou $\left.0<h \leq h_{*}^{(1)}\right\} \quad$ e $\quad\left\{(h, 0) \mid-h_{*}^{(1)}<h<0\right.$ ou $\left.h>h_{*}^{(1)}\right\}$

pertencem ao domínio de atração de $E_{+}$e $E_{-}$, respectivamente.

ii. Se $\alpha=\alpha^{*}$

então $E_{+}$e $E_{-}$são instáveis.

(b) $\alpha^{*}<\alpha \leq 1$

então $\left(H_{t}, \bar{A}_{t}\right)_{t \geq 0}$ não possui equilíbrios estáveis. Além disso, se $\left(H_{0}, \bar{A}_{0}\right)$ não for um equilíbrio instável, vale:

i. $|\mathcal{T}|=\infty$;

ii. $\exists \tau_{\lambda} \in \mathbb{N}:\left|H_{\tau}\right|>h_{*} \forall \tau \in \mathcal{T} \operatorname{com} \tau>\tau_{\lambda}$,

onde $\mathcal{T}=\left\{\tau \in \mathbb{N} \mid \bar{A}_{\tau} \cdot \bar{A}_{\tau+1} \leq 0\right\}$ denota o conjunto dos tempos de reversão de sinal de $\left(\bar{A}_{t}\right)_{t \geq 0}$.

A prova da Proposição 6 é análoga à prova da Proposição 4.

\subsubsection{Interpretação socioeconômica: estabilidade local de dois preços de equilíbrio de mercado}

A principal diferença entre o caso analisado na Seção $2.4 .2(J \geq 0$ e $K=0)$ e o caso da presente seção, $J=0$ e $K \geq 0$, está na possibilidade de ocorrência de dois equilíbrios localmente estáveis, $E_{+}$e $E_{-}$, diferentes de $(0,0)$. Este fato decorre da presença de especuladores interagindo diretamente entre si $(K>0)$. Esta interação implica na ocorrência de um excesso de demanda positivo e estável por parte dos especuladores, que é contraposto por um excesso de oferta (excesso de demanda negativo) em igual valor absoluto por parte dos fundamentalistas. Essa contraposição do excesso de demanda, por parte de especuladores, e excesso de oferta, por parte de fundamentalistas, ocorre a um nível $\left[\bar{A}_{+}\right]^{-1}(0)>0$ da sobrevalorização do ativo que não excede o limiar de reversão $h_{*}^{(1)}$ $\left(0<\left[\bar{A}_{+}\right]^{-1}(0)<h_{*}^{(1)}\right)$. Este fato decorre da presença moderada de especuladores no mercado $\left(0<\alpha<\alpha_{*}\right)$ e faz com que o equilíbrio alcançado seja localmente estável.

Observamos que os preços de equilíbrio de mercado correspondentes ao equilíbrios $E_{+}$ e $E_{-}$são dados por $\bar{P}_{+}:=\bar{P}+\left[\bar{A}_{+}\right]^{-1}(0)$ e $\bar{P}_{-}:=\bar{P}_{+}\left[\bar{A}_{-}\right]^{-1}(0)$. Estes preços de equilíbrio 
de mercado são localmente estáveis e estão dispostos simetricamente acima e abaixo da avaliação individual média sobre o valor fundamental do ativo $\bar{P}$.

Para uma população grande, porém, finita de agentes, esperamos uma flutuação do preço em torno do preço de equilíbrio localmente estável $\bar{P}_{+}$cujo tempo de flutuação é estocástico e finito; assim que o preço $P_{t}$ ultrapassar o limiar $P_{+}:=\bar{P}+h_{*}^{(1)}>\bar{P}_{+}$, o preço $P_{t}$ será atraído para o outro preço de equilíbrio $\bar{P}_{-}$, e vice-versa. Pela construção do processo, conjecturamos que, para uma população finita de agentes, os tempos de flutuação em torno de cada preço de equilíbrio sejam variáveis aleatórias independentes e identicamente distribuídas segundo uma distribuição de probabilidades próxima à distribuição exponencial. É fácil ver também que, quanto maior for o número de agentes $N$, maior será o tempo de flutuação do preço $P_{t}$ em torno de cada preço de equilíbrio (veja a Figura 2.16).

Os resultados expostos no parágrafo anterior dizem respeito ao comportamento do processo para uma quantidade finita de agentes. Estes resultados não foram formulados e provados rigorosamente. Apresentaremos na seção seguinte quatro simulações do processo que evidenciam as conjecturas feitas acima. Gostaríamos de pontuar que os resultados formalmente formulados e provados dizem respeito a uma quantidade infinita de agentes. Para uma quantidade infinita de agentes e sob $(\Phi(0))^{-1}<2 K, 0<\alpha \leq \alpha_{*}$ e $\lambda$ suficientemente pequeno, podemos concluir, a partir da Proposição 6, que o preço $P_{t}$ convergirá deterministicamente para um dos preços de equilíbrio de mercado localmente estáveis $\bar{P}_{-}$ ou $\bar{P}_{+}$, dependendo da condição inicial $\left(H_{0}, \bar{A}_{0}\right)$. Os resultados com relação ao comportamento do sistema dinâmico do preço $\left(P_{t}\right)_{t \geq 0}$ nos demais casos, i.e., no caso $(\Phi(0))^{-1}<2 K$ e $\alpha>\alpha_{*}$ e no caso $(\Phi(0))^{-1}>2 K$, podem ser igualmente derivados da Proposição 6 . Nestes casos, o sistema dinâmico do preço apresenta dois comportamentos já estudados anteriormente: no caso $(\Phi(0))^{-1}<2 K$ e $\alpha>\alpha_{*}$, bolhas especulativas de mercado e crashes de preço do ativo se alternam sucessivamente; e no caso $(\Phi(0))^{-1}>2 K$, o preço $P_{t}$ converge para a avaliação individual média sobre o valor fundamental do ativo $\bar{P}$.

Vamos mostrar agora como uma intervenção no sistema pode alterar o preço de equilíbrio de mercado do ativo. Para tanto, vamos observar o sistema de preço e excesso de demanda $\left(P_{t}, \bar{A}_{t}\right)_{t \geq 0}$. Observemos inicialmente que $\left(P_{t}, \bar{A}_{t}\right)=\left(\bar{P}+H_{t}, \bar{A}_{t}\right), t=0,1,2, \ldots$ Como segue da Proposição 6, sob $\left(\Phi^{\prime}(0)\right)^{-1}<2 K$ e $0<\alpha<\alpha_{*}$, o sistema $\left(H_{t}, \bar{A}_{t}\right)_{t \geq 0}$ 


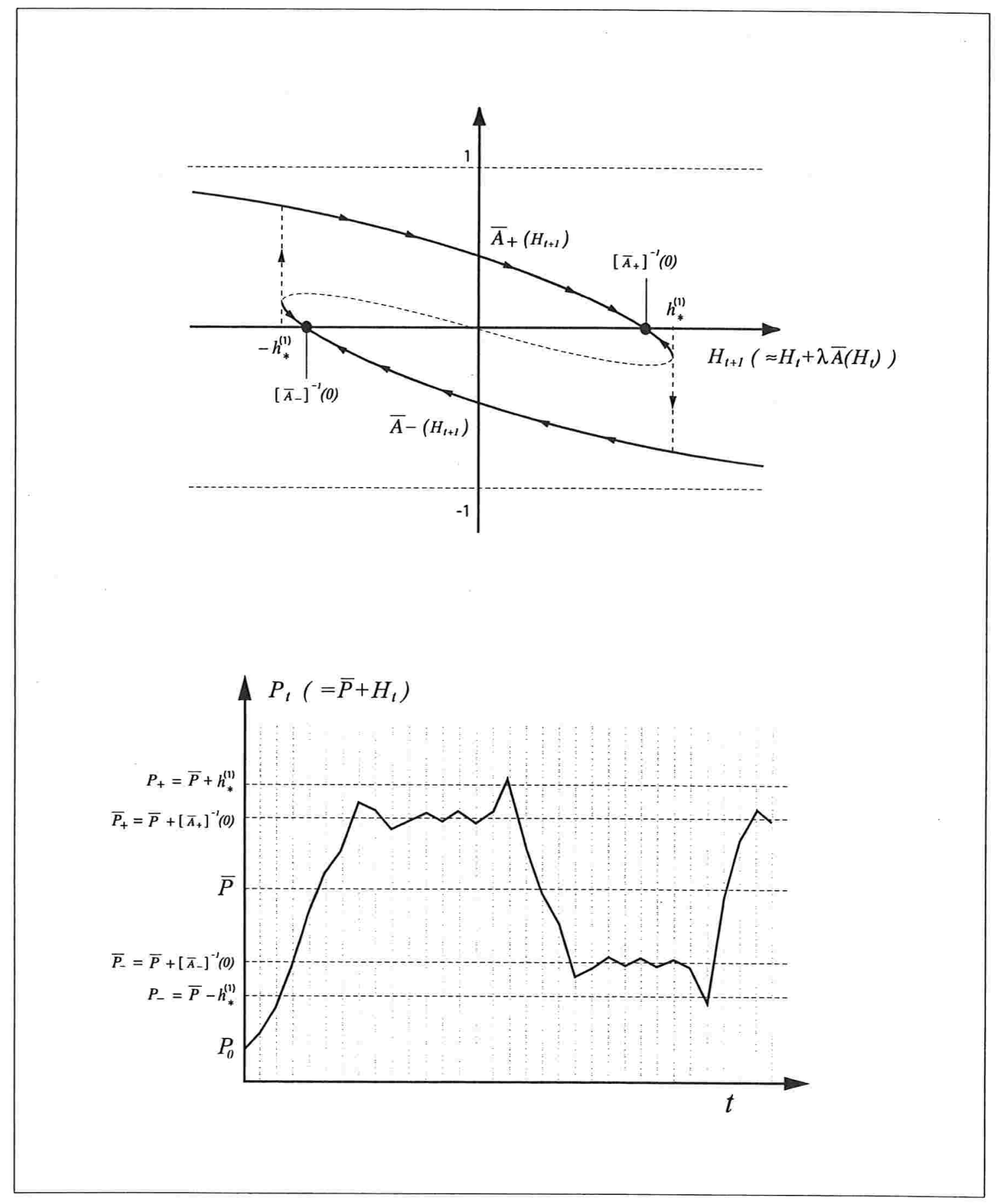

Figura 2.16: Caso $\left(\Phi^{\prime}(0)\right)^{-1}<2 K$ e $0<\alpha<\alpha_{*}$. O primeiro gráfico acima ilustra as convergências de $\left(H_{t}, \bar{A}_{t}\right)$ para os equilíbrios $\left(\left[\bar{A}_{-}\right]^{-1}(0), 0\right)$ e $\left(\left[\bar{A}_{+}\right]^{-1}(0), 0\right)$. O segundo gráfico ilustra o comportamento do preço $P_{t}$ para uma população grande, porém, finita de agentes. 


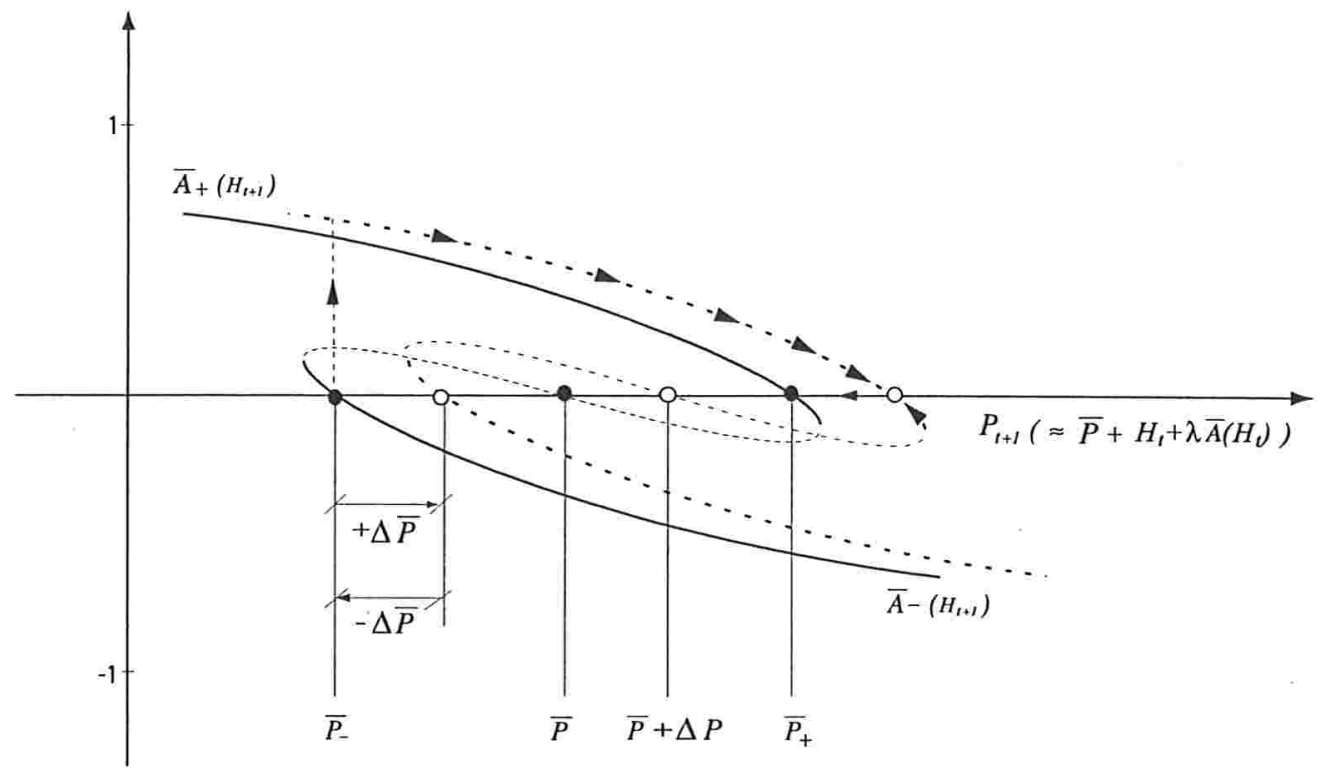

Figura 2.17: Caso $\left(\Phi^{\prime}(0)\right)^{-1}<2 K$ e $0<\alpha<\alpha_{*}$. Ilustra uma intervenção no sistema de preço e excesso de demanda $\left(P_{t}, \bar{A}_{t}\right)_{t \geq 0}$ que muda o preço de equilíbrio de mercado de $\bar{P}_{-}$ para $\bar{P}_{+}$.

possui dois outros equilíbrios, $E_{+}$e $E_{-}$, diferentes de $(0,0)$. É fácil ver que os domínios de atração dos equilíbrios localmente estáveis de $\left(P_{t}, \bar{A}_{t}\right)_{t \geq 0}$, dados por $\left(\bar{P}+\left[\bar{A}_{-}\right]^{-1}(0), 0\right)$ e $\left(\bar{P}+\left[\bar{A}_{+}\right]^{-1}(0), 0\right)$, são translações contínuas em $\bar{P}$ dos domínios de atração dos respectivos equilíbrios localmente estáveis de $\left(H_{t}, \bar{A}_{t}\right)_{t \geq 0}$, dados por $\left(\left[\bar{A}_{-}\right]^{-1}(0), 0\right)$ e $\left(\left[\bar{A}_{+}\right]^{-1}(0), 0\right)$. Este fato sugere que podemos induzir uma mudança do preço de equilíbrio de mercado de $\bar{P}_{-}$para $\bar{P}_{+}$mediante uma variação de $\bar{P}$. Para tanto, basta aumentar $\bar{P}$ por um incremento positivo $\Delta \bar{P}$ suficientemente grande, i.e., $\Delta \bar{P}>h_{*}^{(1)}-\left[\bar{A}_{+}\right]^{-1}(0)$, e, posteriormente, reduzir vagarosamente a avaliação individual média sobre o valor fundamental do ativo a seu nível original em $\bar{P}$ (veja a Figura 2.17).

$\mathrm{Na}$ vida real, tal intervenção poderia ser alcançada pela divulgação de boas notícias sobre mudanças nos fundamentos econômicos do ativo que, posteriormente, poderiam ser relativizadas na medida em que os fatos se mostrarem contrários às notícias. Esta possibilidade é interessante por sugerir que, muitas vezes, "boas notícias", mesmo sem fundamentos em fatos reais, podem decisivamente mudar o preço de equilíbrio de mercado do ativo. Ou seja, uma vez ocorrida a mudança de equilíbrio, a realidade pode se revelar vagarosamente contrária às notícias dadas, sem que o novo regime de equilíbrio $\bar{A}_{+}(\cdot)$ seja 
abandonado. De forma análoga, este mecanismo também explica porque, muitas vezes, uma afirmação infeliz feita por um político pode derrubar o preço de um ativo financeiro. Nestes casos, podemos observar que, muitas vezes, o preço do ativo permanece flutuando em torno de um patamar inferior, mesmo quando tal afirmação venha a ser reformulada publicamente. Um exemplo deste fenômeno ocorreu quando o atual presidente dos EUA, George W. Bush, em entrevista coletiva conjunta com o primeiro-ministro japonês Junichiro Koizumi em fevereiro de 2002, pretendia comentar a deflação da moeda japonesa. Em sua fala, Bush usou erroneamente o termo "desvalorização", querendo dizer "deflação". A reação do mercado de câmbio foi imediata: desvalorização do iene face ao dólar. O iene não se recuperou imediatamente, mesmo após o deslize do presidente ter sido esclarecido.

Observamos, por fim, que este tipo de fenômeno não ocorre se não houver especuladores no mercado ou se a heterogeneidade das avaliações individuais sobre o valor fundamental do ativo for relativamente alta. Nestas condições, i.e., sob $\left(\Phi^{\prime}(0)\right)^{-1} \geq 2 K$ ou $\alpha=0$, só haverá um preço de equilíbrio de mercado correspondendo à avaliação individual média sobre o valor fundamental do ativo $\bar{P}$. Neste caso, uma intervenção que pretenda simplesmente "motivar" o mercado com notícias sem fundamentos em fatos reais, resultaria apenas em uma translação temporária do único preço de equilíbrio de mercado globalmente estável. Na medida em que os fatos se revelarem contrários às notícias, o preço de equilíbrio de mercado retorna à sua posição original (veja a Figura 2.18). Neste caso, tal intervenção resultaria apenas em um efeito negativo sobre a credibilidade de "boas notícias". 


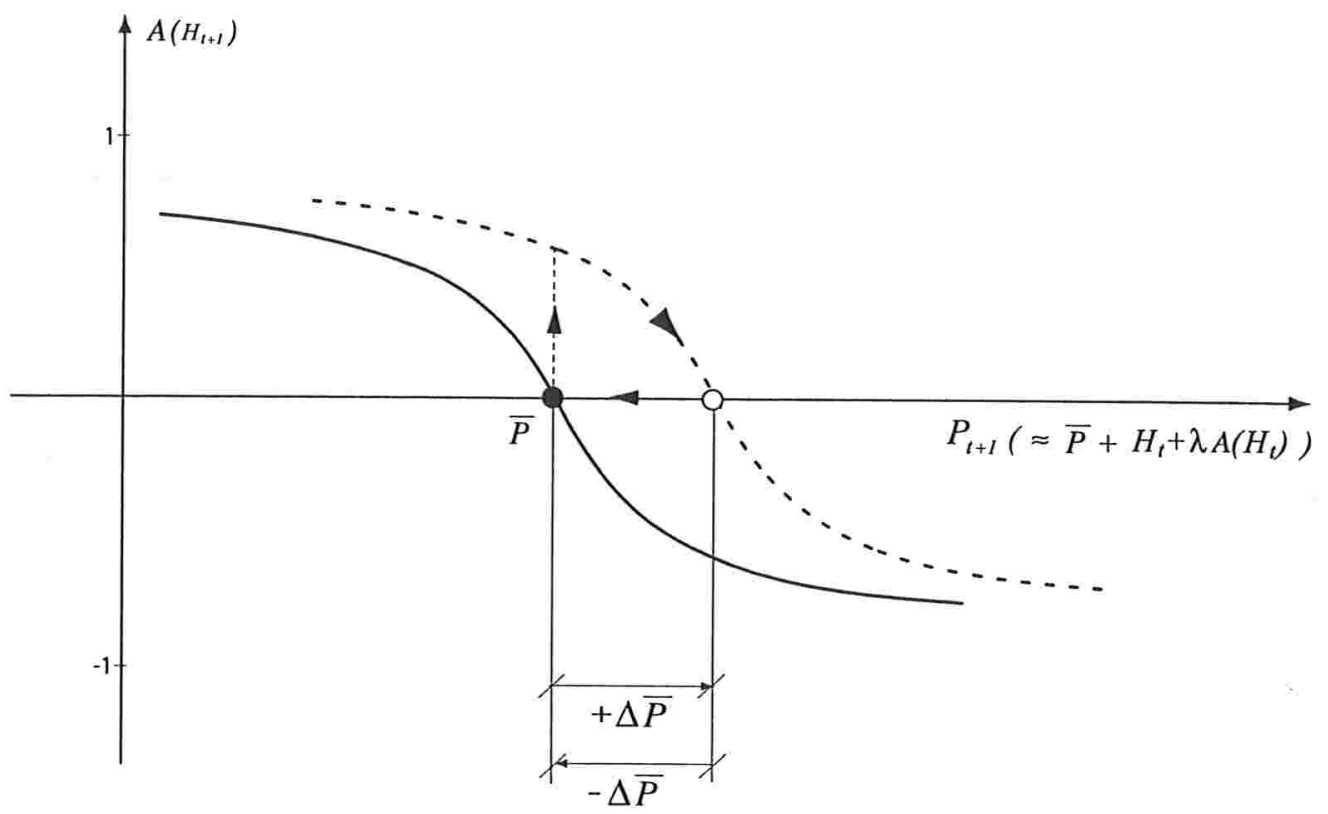

Figura 2.18: Caso $\left(\Phi^{\prime}(0)\right)^{-1}>2 K$ ou $\alpha=0$. Ilustra uma intervenção no sistema de preço e excesso de demanda $\left(P_{t}, \bar{A}_{t}\right)_{t \geq 0}$ que não altera o preço de equilíbrio em $\bar{P}$. 


\subsubsection{Simulação}

Nesta seção apresentaremos quatro simulações do processo estocástico da sobrevalorização e excesso de demanda do ativo $\left(H_{t}, \bar{A}_{t}\right)_{t \geq 0}$. Estas simulações contemplam o caso $J=0$, $\left(\Phi^{\prime}(0)\right)^{-1}<2 K$ e $\lambda>0$ para diferentes valores de $\alpha$. Lembramos que este é o caso em que o sistema dinâmico correspondente $\left(H_{t}^{(\infty)}, \bar{A}_{t}^{(\infty)}\right)_{t \geq 0}$, i.e., o processo $\left(H_{t}, \bar{A}_{t}\right)_{t \geq 0}$ para um número infinito de agentes, pode possuir dois equilíbrios localmente estáveis diferentes de $(0,0)$. Lembramos ainda que a ocorrência destes equilíbrios depende do parâmetro $\alpha$ (fração de especuladores no mercado). Para cada uma das quatro simulações, usamos um valor diferente para $\alpha \in[0,1]$ :
1) $\alpha=0,8$
2) $\alpha=0,5$
3) $\alpha=0,297$
4) $\alpha=0,2$

Todos os demais parâmetros são os mesmos para as quatro simulações:

$$
\begin{aligned}
& \Phi=N(0,1) \quad-\text { Função de distribuição dos desvios das avaliações individuais } \\
& \text { Função de distribuição acumulada da Normal Padrão } \\
& K=2,00 \quad-\text { Influência social direta } \\
& J=0,00-\text { Influência social de preço } \\
& N=10000,00-\text { Número total de agentes } \\
& \lambda=0,10-\text { Ajuste do preço } \\
& T=3000,00-\text { Tempo de simulação }
\end{aligned}
$$

Para $\Phi$ e $K$ acima mencionados, calculamos numericamente o valor da fração crítica de especuladores mediante a fórmula (2.99). Obtemos

$$
\alpha_{*}=0,300587 \simeq 0,30
$$

A Figura 2.19 mostra o gráfico das quatro simulações do processo $\left(H_{t}, \bar{A}_{t}\right)_{t \geq 0}$ para os valores de $\alpha$ indicados em (2.101). A simulação 1) $\alpha=0,8$, corresponde ao ciclo mais aberto. A simulação 2) $\alpha=0,5$, corresponde ao segundo ciclo, contado do ciclo mais aberto para o mais fechado. A simulação 3) $\alpha=0,297$, corresponde ao ciclo interior ao ciclo da simulação 2). A simulação 4) $\alpha=0,2$, corresponde ao acúmulo de pontos 
próximo ao acúmulo de pontos direito pertencente ao ciclo da simulação 3). A origem $(0,0)$ é o ponto de partida comum a todas as simulações, i.e., $\left(H_{0}, \bar{A}_{0}\right)=(0,0){ }^{8}$

Chamamos a atenção para a simulação 3) $\alpha=0,297$. Neste caso, $\alpha$ encontra-se um pouco abaixo de $\alpha_{*}=0,3$ (fração crítica de especuladores). Neste caso, o sistema dinâmico $\left(H_{t}^{(\infty)}, \bar{A}_{t}^{(\infty)}\right)_{t \geq 0}$ possui dois únicos equilíbrios localmente estáveis, $E_{-}:=\left(\left[\bar{A}_{-}\right]^{-1}(0), 0\right) \mathrm{e}$ $E_{+}:=\left(\left[\bar{A}_{+}\right]^{-1}(0), 0\right)$. Para esta fração de especuladores, podemos observar que $\left(H_{t}, \bar{A}_{t}\right)$ flutua em torno de cada um destes equilíbrios, até que o mesmo escape para o domínio de atração do outro equilíbrio. Este fato pode ser observado na simulação porque o domínio de atração destes equilíbrios são relativamente pequenos quando $\alpha \lesssim \alpha_{*}$, i.e., quando $\alpha$ está abaixo e próximo de $\alpha_{*}$. Já quando $\alpha=0,2<0,3=\alpha_{*}$, o tempo de espera para a ocorrência do escape do domínio de atração de $\left(\left[\bar{A}_{+}\right]^{-1}(0), 0\right)$ é relativamente grande, i.e., não pode ser observado no tempo de simulação. Como pode ser visto na Figura 2.19, a simulação no caso $\alpha=0,2$ mostra que $\left(H_{t}, \bar{A}_{t}\right)_{t \geq 0}$ é atraído para o equilíbrio $\left(0,\left[\bar{A}_{+}\right]^{-1}\right)$, onde permanece flutuando durante todo o tempo $t=0,1,2, \ldots, T=3000$. Observamos, ainda, que a distância entre os pontos de equilíbrios $E_{-}$e $E_{+}$é uma função crescente e contínua de $\alpha$ para $\alpha \in\left[0, \alpha_{*}\right]$. Quando $\alpha$ tende a zero, os equilíbrios $E_{-}$e $E_{+}$colapsam em $(0,0)$, onde, então, temos um único equilíbrio globalmente estável.

Nos outros dois casos, $\alpha=0,5$ e $\alpha=0,8$, a fração de especuladores está acima da fração crítica $\alpha_{*}=0,3$. Nestes casos, o processo é atraído para ciclos correspondendo à auto-organização de bolhas especulativas e crashes de preço do ativo.

Na Figura 2.20 apresentamos o gráfico do processo da sobrevalorização do ativo $\left(H_{t}\right)_{t \geq 0}$ para $\alpha=0,297 \lesssim 0,3=\alpha_{*}$. Neste gráfico, podemos observar o tempo de flutuação de $H_{t}$ em torno de cada equilíbrio localmente estável $\left[\bar{A}_{-}\right]^{-1}(0)$ e $\left[\bar{A}_{+}\right]^{-1}(0)$. Lembramos que o processo de preço é dado por $P_{t}=\bar{P}+H_{t}$.

Melhoramento da estimativa da probabilidade de desvio entre $W_{t}$ e $w_{t}$. Observamos que a estimativa da probabilidade de desvio entre $W_{t}$ e $w_{t}$, onde $J=0, K \geq 0$ e $\lambda>0$, pode ser melhorada mediante aplicação dos Corolários 1 e 2 da Proposição 1. Isto

\footnotetext{
${ }^{8}$ Apesar de $(0,0)$ ser um equilíbrio do sistema dinâmico $\left(H_{t}^{(\infty)}, \bar{A}_{t}^{(\infty)}\right)_{t \geq 0}$, o processo $\left(H_{t}, \bar{A}_{t}\right)_{t \geq 0}$ escapa de $(0,0)$. Isto se deve ao fato de $(0,0)$ ser um equilíbrio instável de $\left(H_{t}^{(\infty)}, \bar{A}_{t}^{(\infty)}\right)_{t \geq 0}$, por um lado, e, por outro lado, por $\left(H_{t}, \bar{A}_{t}\right)_{t \geq 0}$ ser um processo estocástico, i.e., onde o número de agentes $N(=10000)$ é finito. Isso faz com que $\left(H_{t}, \bar{A}_{t}\right)$ abandone o ponto $(0,0)$ rapidamente.
} 


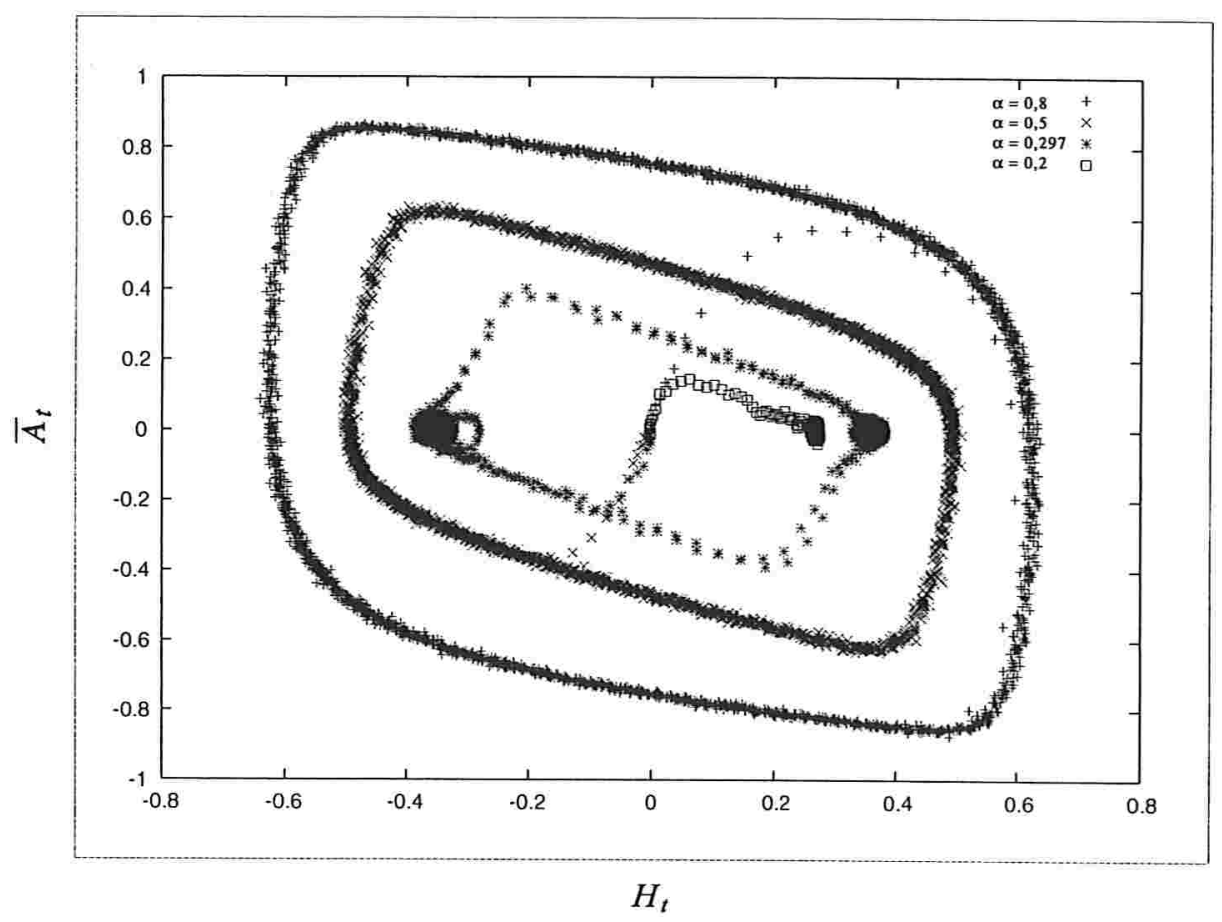

Figura 2.19: Caso $\left(\Phi^{\prime}(0)\right)^{-1}<2 K, J=0$ e $\lambda>0$. Simulação do processo $\left(H_{t}, \bar{A}_{t}\right)_{t \geq 0}$ para diferentes valores de $\alpha$ (fração de especuladores).

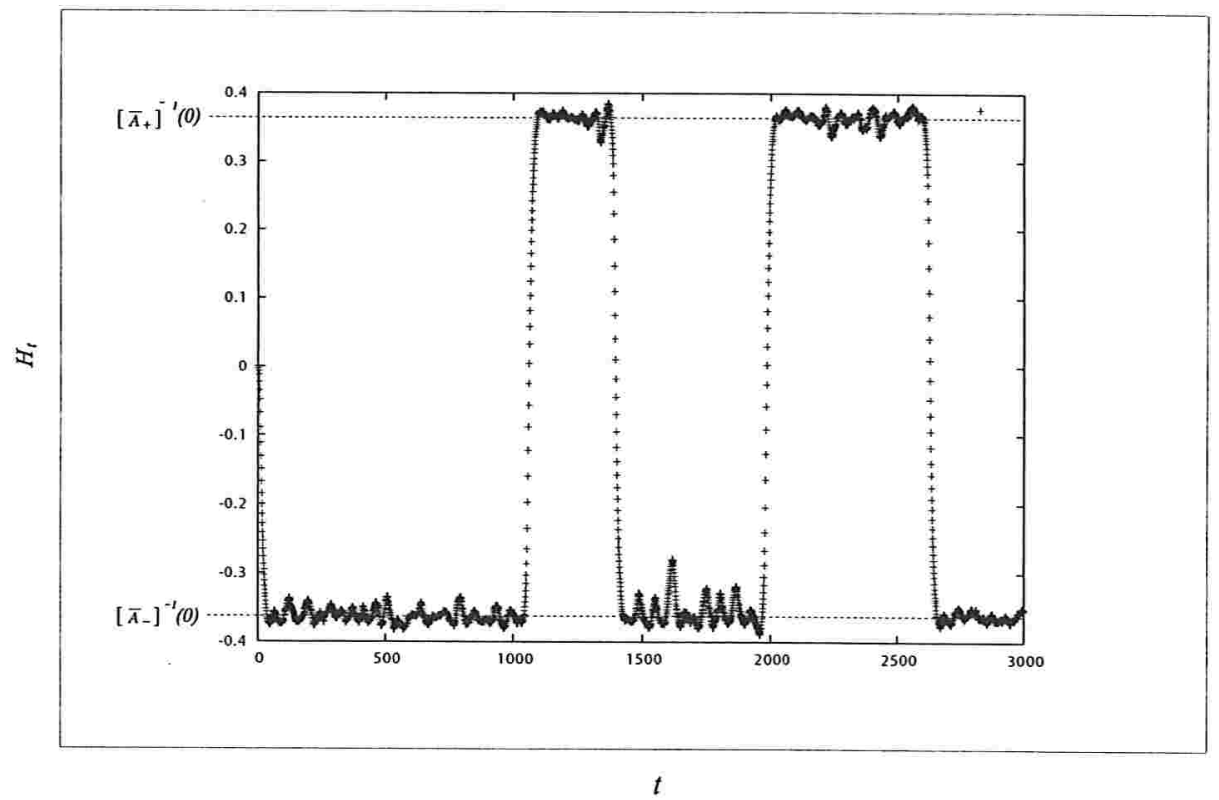

Figura 2.20: Caso $\left(\Phi^{\prime}(0)\right)^{-1}<2 K, J=0$ e $\lambda>0$. Simulação do processo $\left(H_{t}\right)$ para $\alpha=0,297 \lesssim 0,3=\alpha_{*}$ (fração de especuladores próxima à fração crítica). 
é, a cota superior (2.24) para a probabilidade em (2.23), onde $J=0, K \geq 0$ e $\lambda>0$, pode ser substituída por uma estimativa do tipo (2.30) se supusermos que $\left(\Phi^{\prime}(0)\right)^{-1}$ (heterogeneidade das avaliações individuais sobre o valor fundamental do ativo) ou $K$ (influência social direta) seja suficientemente grande. Omitiremos o tratamento desta questão. Observamos apenas que o sentido e a prova desta afirmação são análogos aos correspondentes da seção anterior, onde tratamos a mesma questão para $K=0, J \geq 0$ e $\lambda>0$. 


\subsubsection{J $J \geq 0, K \geq 0, \lambda>0$ (caso geral). Principais resultados}

Nesta seção trataremos o caso misto em que $J \geq 0$ e $K \geq 0$. Observamos que este caso recai em situações já estudadas e interpretadas anteriormente. No entanto, há uma diferença que ressaltaremos nesta seção. Apresentaremos apenas os principais resultados. Trataremos diretamente o caso $\lambda>0$. Estes resultados ainda não foram checados em todos os detalhes e estão apresentados na Conjectura 1. Na Seção 2.4.5.1, comentaremos as principais diferenças entre o caso geral e dois outros casos estudados anteriormente, caso $J \geq 0, K=0$ e caso $J=0, K \geq 0$.

Inicialmente observemos que, no caso geral, a dinâmica de $\left(w_{t}\right)_{t \geq 0}$ é dada por

$$
\left\{\begin{array}{l}
H_{t+1}=H_{t}+\lambda \bar{A}_{t} \\
\bar{S}_{t+1}=2 \Phi\left(J \bar{A}_{t}+K \bar{S}_{t}-H_{t+1}\right)-1 \quad t=0,1,2, \ldots \\
\bar{F}_{t+1}=2 \Phi\left(-H_{t+1}\right)-1
\end{array}\right.
$$

onde $\bar{A}_{t}=\alpha \bar{S}_{t}+(1-\alpha) \bar{F}_{t}, t=0,1,2, \ldots$

A partir do sistema (2.103), podemos derivar o sistema da sobrevalorização e excesso de demanda do ativo $\left(H_{t}, \bar{A}_{t}\right)_{t \geq 0}$. Este sistema é dado pela seguinte interação:

$$
\left(H_{t}, \bar{A}_{t}\right)=\digamma\left(H_{t-1}, \bar{A}_{t-1}\right), \quad t=0,1,2, \ldots
$$

onde $F(H, \bar{A})=(H+\lambda \bar{A}, g(\bar{A} ; H+\lambda \bar{A})$ e $g$ definida por

$$
g(x ; h):=\alpha F((J+K / \alpha) \cdot x+(K(1-\alpha) / \alpha) F(h)-h)+(1-\alpha) F(-h) 2.105)
$$

onde $F:=2 \Phi-1$.

Como nas seções anteriores, definimos os equilíbrios de $\left(H_{t}, \bar{A}_{t}\right)_{t \geq 0}$ como sendo soluções da equação $(\bar{A}, H)=\digamma(\bar{A}, H)$. Estes equilíbrios serão classificados em equilíbrios globalmente estáveis, localmente estáveis e instáveis, da mesma forma como os equilíbrios de $\left(H_{t}, \bar{A}_{t}\right)_{t \geq 0}$ foram classificados nestas categorias na Seção 2.4.2.2.

Para o sistema dinâmico (2.104), temos:

Conjectura 1. Seja $\left(H_{t}, \bar{A}_{t}\right)_{t \geq 0}$ dado por (2.104). Inicialmente, temos que $(0,0)$ é sempre um equilíbrio de $\left(H_{t}, \bar{A}_{t}\right)_{t \geq 0}$. Além disso, existe um número positivo $\lambda_{c}$, tal que, para todo $\lambda$ com $0<\lambda<\lambda_{c}$, vale: 
1. Se $\left(\Phi^{\prime}(0)\right)^{-1}>2(\alpha J+K)$ ou $\quad \alpha=0$

então $(0,0)$ é o único equilibrio globalmente estável de $\left(H_{t}, \bar{A}_{t}\right)$.

2. Se $\left(\Phi^{\prime}(0)\right)^{-1}<2 K$

então $(0,0)$ é um equilíbrio instável de $\left(H_{t}, \bar{A}_{t}\right)_{t \geq 0}$. Para $\left(\Phi^{\prime}(0)\right)^{-1}<2 K$, seja $\alpha_{*}$ dado por (2.99). Se, além de $\left(\Phi^{\prime}(0)\right)^{-1}<2 K$, valer alternativamente:

(a) $0<\alpha \leq \alpha_{*}$

então $\left(H_{t}, \bar{A}_{t}\right)$ possui dois outros equilibrios, denotados por $E_{+} e E_{-}$, onde $E_{-}=-E_{+}, E_{+}:=\left(\bar{H}_{+}, 0\right)$ para um certo $\bar{H}_{+}$, que satisfaz $0<\bar{H}_{+}<h_{*}$, sendo que $h_{*}$ é unicamente determinado pelo seguinte sistema de equações em $h_{*} e \bar{A}_{*}$ :

$$
\bar{A}_{*}=\left.g\left(\bar{A}_{*}, h_{*}\right) \quad e \quad \frac{\partial g\left(x ; h_{*}\right)}{\partial x}\right|_{x=\bar{A}_{*}}=1
$$

onde $g$ é definida por (2.105).

Para os equilibrios $E_{+}$e $E_{-}$, vale:

i. Se $0<\alpha<\alpha^{*}$

então $E_{+}$e $E_{-}$são localmente estáveis, onde

$$
\left\{(h, 0) \mid h<-h_{*} \text { ou } 0<h \leq h_{*}\right\} \quad \text { e } \quad\left\{(h, 0) \mid-h_{*}<h<0 \text { ou } h>h_{*}\right\}
$$

pertencem ao dominio de atração de $E_{+}$e $E_{-}$, respectivamente.

ii. Se $\alpha=\alpha^{*}$

então $E_{+}$e $E_{-}$são instáveis.

(b) $\alpha^{*}<\alpha \leq 1$

então $\left(H_{t}, \bar{A}_{t}\right)_{t \geq 0}$ não possui equilibrios estáveis. Além disso, se $\left(H_{0}, \bar{A}_{0}\right)$ não for um equilibrio instável, vale:

i. $|\mathcal{T}|=\infty$,

ii. $\exists \tau_{\lambda} \in \mathbb{N}:\left|H_{\tau}\right|>h_{*} \forall \tau \in \mathcal{T} \operatorname{com} \tau>\tau_{\lambda}$ e $h_{*}$ determinados por (2.106), onde $\mathcal{T}=\left\{\tau \in \mathbb{N} \mid \bar{A}_{\tau} \cdot \bar{A}_{\tau+1} \leq 0\right\}$ denota o conjunto dos tempos de reversão de sinal de $\left(\bar{A}_{t}\right)_{t \geq 0}$. 
3. Se $2 K \leq\left(\Phi^{\prime}(0)\right)^{-1}<2(J \alpha+K)$

então vale o mesmo que em (2b).

\subsubsection{Principais diferenças entre o caso geral $J \geq 0, K \geq 0$ e os casos $J \geq 0$, $K=0$ e $J=0, K \geq 0$}

No caso misto, existem três faixas de valores para o parâmetro $\left(\Phi^{\prime}(0)\right)^{-1}$ (heterogeneidade das avaliações individuais sobre o valor fundamental do ativo): baixa, $\left(\Phi^{\prime}(0)\right)^{-1}<2 K$; média, $2 K \leq\left(\Phi^{\prime}(0)\right)^{-1}<2(\alpha J+K)$; e alta, $2(\alpha J+K) \leq\left(\Phi^{\prime}(0)\right)^{-1}$. Somente sob baixa heterogeneidade das avaliações individuais sobre o valor fundamental do ativo, $\left(\Phi^{\prime}(0)\right)^{-1}<2 K$, e, quando a quantidade de especuladores for suficientemente pequena, $\alpha<\alpha_{*}$, o sistema $\left(H_{t}, \bar{A}_{t}\right)_{t \geq 0}$ possui dois equilíbrios localmente estáveis, $E_{+}$e $E_{-}$, diferentes de $(0,0)$. Sob média heterogeneidade das avaliações individuais sobre o valor fundamental do ativo, $\left(H_{t}, \bar{A}_{t}\right)_{t \geq 0}$ não possui equilíbrios estáveis. Neste caso, o sistema $\left(H_{t}, \bar{A}_{t}\right)_{t \geq 0}$ apresenta formação de bolhas e crashes de preço do ativo no sentido já interpretado anteriormente. Observamos que, para esta faixa intermediária, $2 K \leq\left(\Phi^{\prime}(0)\right)^{-1}<2(\alpha J+K)$, a fração crítica de especuladores não está definida (a definição de $\alpha_{*}$ em (2.99) pressupõe $\left.2 K>\left(\Phi^{\prime}(0)\right)^{-1}\right)$. Quando $2 K \leq\left(\Phi^{\prime}(0)\right)^{-1}<2(\alpha J+K)$, só há formação de bolhas especulativas e crashes de preço do ativo para qualquer $\alpha>0$. Isto decorre do fato de as avaliações individuais sobre o valor fundamental do ativo serem muito heterogêneas em relação ao grau de influência social direta entre especuladores $K$. A existência dos dois equilíbrios de mercado localmente estáveis, $E_{+}$e $E_{-}$, pressupõe uma influência social direta entre especuladores (parâmetro $K$ ) relativamente grande. Analogamente às seções anteriores, sob alta heterogeneidade das avaliações individuais sobre o valor fundamental do ativo, $2(\alpha J+K)<\left(\Phi^{\prime}(0)\right)^{-1}$, o sistema $\left(H_{t}, \bar{A}_{t}\right)_{t \geq 0}$ possui um único equilíbrio globalmente estável em $(0,0)$.

Observamos, por fim, que o comportamento do sistema $\left(H_{t}, \bar{A}_{t}\right)_{t \geq 0}$ no caso misto, i.e., no caso em que $J \geq 0$ e $K \geq 0$, recai em um dos casos já estudados anteriormente: ou no caso $J \geq 0$ e $K=0$ ou no caso $J=0$ e $K \geq 0$. Em qual dos casos se enquadra o comportamento do sistema $\left(H_{t}, \bar{A}_{t}\right)_{t \geq 0}$, depende da relação entre os parâmetros $K$ e $\left(\Phi^{\prime}(0)\right)^{-1}$. Se $2 K \leq\left(\Phi^{\prime}(0)\right)^{-1}$, o sistema $\left(H_{t}, \bar{A}_{t}\right)_{t \geq 0}$ se comporta como no caso $J \geq 0$ e $K=0$. Se $2 K>\left(\Phi^{\prime}(0)\right)^{-1}$, o sistema $\left(H_{t}, \bar{A}_{t}\right)_{t \geq 0}$ se comporta como no caso $J=0$ e $K \geq 0$. 


\section{Capítulo 3}

\section{Modelo de Influência Social Estocástica}

\subsection{Definição do modelo}

Nesta seção modificaremos o modelo apresentado no Capítulo 2 a fim de permitir que especuladores sejam heterogêneos quanto à intensidade com que são influenciados pela opinião de outros agentes e pela tendência do preço.

\subsubsection{Regras de decisão}

Seguiremos com a mesma regra de compra para fundamentalistas apresentada em (2.9). A nova regra para os especuladores é dada por

$X_{t}^{(s)}=\left\{\begin{array}{ll}+1 & \text { se } \\ -1 & \text { caso contrário }\end{array} J_{t}^{(s)} \bar{A}_{t-1}+K_{t}^{(s)} \bar{S}_{t-1}-\left(P_{t}-\bar{P}\right)>0 \quad \forall s \in S, \forall t=1,2, \ldots\right.$

onde $J_{t}^{(s)}$ e $K_{t}^{(s)}$ são as influências sociais de preço e influências sociais diretas específicas de cada especulador $s \in S$, distribuídas segundo uma função de distribuição de probabilidades a ser descrita a seguir.

Notamos que a única diferença entre este novo modelo e o modelo apresentado no Capítulo 2 está na substituição da regra (2.12) pela nova regra (3.1). Todas as demais definições do modelo continuam inalteradas. Em particular, a regra de decisão fundamentalista continua sendo a mesma regra apresentada em (2.9). Apresentaremos mais uma vez a probabilidade de compra de um fundamentalista $Q_{F}: \mathbb{R} \times[-1,1] \rightarrow \mathbb{R} \times[-1,1]$ derivada da regra (2.9). Seja $w=(H, \bar{S}, \bar{F}) \in \mathbb{R} \times[-1,1]^{2}$, lembramos que a probabilidade 
de compra de um fundamentalista, derivada de (2.9), é dada por

$$
Q_{F}(w)=\Phi(-(H+\lambda \bar{A}))
$$

onde $\bar{A}=\alpha \bar{S}+(1-\alpha) \bar{F}$.

\subsubsection{Heterogeneidade das influências sociais $J_{t}^{(s)}$ e $K_{t}^{(s)}, s \in S$, $t \geq 0$}

Neste modelo analisaremos dois casos:

1. $K_{t}^{(s)}=0, \forall s \in S, \forall t \geq 0.1 / J_{t}^{(s)}, s \in S, t \geq 0$ são variáveis aleatórias positivas, independentes e identicamente distribuídas segundo uma função de distribuição de probabilidades acumulada, denotada por $\Psi$.

2. $J_{t}^{(s)}=0, \forall s \in S, \forall t \geq 0.1 / K_{t}^{(s)}, s \in S, t \geq 0$ são variáveis aleatórias positivas, independentes e identicamente distribuídas segundo uma função de distribuição de probabilidades acumulada, denotada por $\Psi$.

Em ambos os casos, a função de distribuição de probabilidades acumulada $\Psi$ possui as seguintes propriedades: $\Psi$ é diferenciável; e, para a derivada de $\Psi$, denotada por $\Psi^{\prime}$, vale:

1. $\Psi^{\prime}$ é contínua em todo $\mathbb{R}$.

2. $\Psi^{\prime} \equiv 0$ em $(-\infty, 0]$.

3. existe $x_{\max }>0$, tal que $\Psi^{\prime}$ é estritamente crescente em $\left(0, x_{\max }\right]$ e estritamente decrescente em $\left(x_{\max }, \infty\right)$.

Focamos na distribuição de probabilidades de $1 / J$ e $1 / K$ (ao invés de $J$ e $K$ ), porque desta forma podemos obter facilmente o sistema dinâmico da sobrevalorização e excesso de demanda do ativo $\left(H_{t}, \bar{A}_{t}\right)_{t \geq 0}$.

\subsubsection{Justificativa para a substituição de $P_{t}^{(s)}$ por $\bar{P}$}

Chamamos a atenção para a diferença entre a regra de decisão especulativa dada (2.12) e a nova regra (3.1). Note que esta diferença não se restringe somente à substituição de $J$ 
e $K$ pelas variáveis aleatórias $J_{t}^{(s)}$ e $K_{t}^{(s)}$, respectivamente; note que a variável aleatória $P_{t}^{(s)}$ foi substituída por $\bar{P}$, i.e., pelo seu valor esperado.

As razões para a substituição $P_{t}^{(s)}$ por $\bar{P}$ são: em primeiro lugar, ainda não investigamos as propriedades matemáticas do modelo quando tanto as influências sociais $\left(J_{t}^{(s)} \mathrm{e}\right.$ $\left.K_{t}^{(s)}, s \in S, t \geq 0\right)$ quanto as avaliações individuais sobre o valor fundamental do ativo $\left(P_{t}^{(s)}, s \in S, t \geq 0\right)$ são variáveis aleatórias. Sob a alteração que apresentamos, obtemos, no entanto, um comportamento diferenciado do modelo, não trivial e interessante do ponto de vista matemático e socioeconômico. Em segundo lugar, a substuição de $P_{t}^{(s)}$ por $\bar{P}$ tem uma justificativa socioeconômica intrínseca aos pressupostos do modelo. Esta justificativa será apresentada agora. Lembramos que, no modelo apresentado no capítulo anterior, a regra de decisão especulativa foi derivada a partir do pressuposto de que especuladores compram (vendem) unidades do ativo, sempre que estimam que seu preço sobe (desce) no instante seguinte (veja a Seção 2.1.4.2). Lembramos também que, de acordo com este pressuposto, havíamos justificado o termo $P_{t}-P_{t}^{(s)}$ na regra de decisão (2.12) como uma forma de o especulador $s \in S$ estimar o impacto que as decisões de compra e venda de fundamentalistas exercem sobre o excesso de demanda resultante (diretamente proporcional ao incremento de preço). Ocorre que o excesso de demanda de fundamentalistas $\bar{F}_{t}\left(=(1-\alpha) 2 \Phi\left(-H_{t}\right)-1\right)$ é uma função decrescente da sobrevalorização do ativo $H_{t}=P_{t}-\bar{P}$. Por conseqüência, se a média das avaliações individuais sobre o valor fundamental do ativo $\bar{P}$ for conhecida, é natural que cada especulador $s \in S$ leve em conta a sobrevalorização do ativo $P_{t}-\bar{P}$, ao invés de $P_{t}-P_{t}^{(s)}$. Note que este não é o caso dos fundamentalistas. A estimativa $P_{t}^{(f)}$, que cada fundamentalista $f \in F$ faz para tomar sua decisão de compra ou venda, não tem a finalidade de estimar a atitude de outros agentes, mas sim de avaliar o valor fundamental do ativo de acordo com suas próprias conviç̧ões sobre os fundamentos econômicos deste ativo. Posto isso, caso a informação $\bar{P}$ esteja disponível, é razoável considerar o modelo segundo a regra de decisão fundamentalista (2.9) e a nova regra de decisão especulativa (3.1).

\subsection{Convergência de $\left(W_{t}\right)_{t \geq 0}$ para $\left(w_{t}\right)_{t \geq 0}$}

Neste modelo analisaremos somente o caso em que $J_{t}^{(s)} \geq 0, K_{t}^{(s)}=0, s \in S$, quase certamente e o caso em que $J_{t}^{(s)}=0, K_{t}^{(s)} \geq 0, s \in S$, quase certamente. Como veremos a 
seguir, em ambos os casos a probabilidade de compra de especuladores $Q_{S}: \mathbb{R} \times[-1,1]^{2} \rightarrow$ $[0,1]$, deduzida a partir da regra (3.1), apresenta pontos de descontinuidade. A descontinuidade de $Q_{S}$ implica, por sua vez, na descontinuidade da função $G$ definida em (2.21) quando $\alpha>0$. Assim sendo, a convergência em probabilidade de $\left(W_{t}\right)_{t \geq 0}$ para $\left(w_{t}\right)_{t \geq 0}$ terá de ser analisada para cada órbita de $\left(w_{t}\right)_{t \geq 0}$, onde $w_{t}=\left(H_{t}, \bar{S}, \bar{F}\right), t=0,1,2, \ldots$, são definidos em (2.20) para a nova probabilidade de compra de especuladores deduzida a partir da nova regra de decisão especulativa (3.1) e para a probabilidade de compra de fundamentalistas relembrada em (3.2). Lembramos que somente poderemos aplicar a Proposição 1 para órbitas de $\left(w_{t}\right)_{t \geq 0}$, onde $w_{t} \in D, t=0,1,2, \ldots$, lembrando que $D$ denota o conjunto dos pontos, onde $G$ é contínua segundo Lipschitz no sentido (2.22). Para a comodidade do leitor, apresentamos mais uma vez a função $G: \mathbb{R} \times[-1,1]^{2} \rightarrow[-1,1]^{2}$. Para $w=(H, \bar{S}, \bar{F}) \in \mathbb{R} \times[-1,1]^{2} \rightarrow[-1,1]^{2}$, temos

$$
G(w):=\left(E_{S}(w), E_{F}(w)\right)
$$

onde

$E_{S}\left(w_{t-1}\right):=\left\{\begin{array}{ll}2 Q_{S}\left(w_{t-1}\right)-1 & \text { se } \alpha>0 \\ 0 & \text { se } \alpha=0\end{array} ; \quad E_{F}\left(w_{t-1}\right):= \begin{cases}2 Q_{F}\left(w_{t-1}\right)-1 & \text { se }(1-\alpha)>0 \\ 0 & \text { se }(1-\alpha)=0\end{cases}\right.$

onde agora $Q_{S}$ será deduzida a partir da nova regra de decisão especulativa (3.1) e onde $Q_{F}$ está sendo relembrada em (3.2).

Caso $\alpha=0$, temos que $G$ é localmente contínua segundo Lipschitz no sentido (2.22) em todo seu domínio de definição. Isto decorre do fato de $Q_{F}$, relembrada em (3.2), ser diferenciável em todo seu domínio de definição. Para $\alpha>0$, a função $G$ reduz-se a $G(w)=\left(2 Q_{S}(w)-1, E_{F}(w)\right)$. Como $E_{F}$ é diferenciável em todo seu domínio de definição, precisamos garantir apenas que $Q_{S}$ seja diferenciável em $w_{t}(t=0,1,2, \ldots)$ para $\alpha>0$. Ou seja, $G$ será localmente contínua segundo Lipschitz em $w_{t}(t=0,1,2, \ldots)$ no sentido (2.22) se $Q_{S}$ for diferenciável em $w_{t}(t=0,1,2, \ldots)$ para $\alpha>0$. Este fato permite que apliquemos a Proposição 1 para garantir a convergência em probabilidade de $W_{t}$ para $w_{t}$, desde que $Q_{S}$ seja diferenciável em $w_{t}(t=0,1,2, \ldots)$ para $\alpha>0$.

Nas duas seções que seguem, determinaremos as regiões dos parâmetros do modelo para as quais a probabilidade de compra de especuladores $Q_{S}$ seja diferenciável em $w_{t}$ $(t=0,1,2, \ldots)$ no caso $J_{t}^{(s)} \geq 0, K_{t}^{(s)}=0, \alpha>0$, e no caso $J_{t}^{(s)}=0, K_{t}^{(s)} \geq 0, \alpha>0$, 
respectivamente. Para estas regiões dos parâmetros do modelo valerá então a convergência em probabilidade de $W_{t}$ para $w_{t}$ quando o número de agentes tende ao infinito.

\subsubsection{Convergência de $\left(W_{t}\right)_{t \geq 0}$ para $\left(w_{t}\right)_{t \geq 0}$. Caso $J_{t}^{(s)}>0$ e $K_{t}^{(s)}=$ $0, s \in S, t \geq 0$}

Provaremos aqui a convergência em probabilidade de $\left(W_{t}\right)_{t \geq 0}$ para $\left(w_{t}\right)_{t \geq 0}$ no caso em que não há influência social direta $\left(K_{t}^{(s)}=0, s \in S\right)$. Neste caso, levando em conta a nova regra de decisão especulativa dada em (3.1), a probabilidade de compra de especuladores $Q_{S}: \mathbb{R} \times[-1,1]^{2} \rightarrow[0,1]$ é dada como segue abaixo. Seja $w=(H, \bar{S}, \bar{F}) \in \mathbb{R} \times[-1,1]^{2}$, então

$$
Q_{S}(w)=\left\{\begin{array}{llll}
\Psi(\bar{A} /(H+\lambda \bar{A})) & \text { se } H+\lambda \bar{A}>0 & \\
1-\Psi(\bar{A} /(H+\lambda \bar{A})) & \text { se } H+\lambda \bar{A}<0 & \\
1 & \text { se } H+\lambda \bar{A}=0 \text { e } \bar{A}>0 \\
0 & \text { se } H+\lambda \bar{A}=0 \text { e } \bar{A} \leq 0
\end{array}\right.
$$

onde $\bar{A}:=\alpha \bar{S}+(1-\alpha) \bar{F}$.

Como já havíamos explicado anteriormente, para garantir a convergência em probabilidade de $\left(W_{t}\right)_{t \geq 0}$ para $\left(w_{t}\right)_{t \geq 0}$, basta garantir que $Q_{S}$, definida agora em (3.4), seja diferenciável em $w_{t}(t=0,1,2, \ldots)$ para $\alpha>0$, onde $\left(w_{t}\right)_{t \geq 0}$ é definido pelo sistema dinâmico (2.20), para as probabilidades de compra de especuladores (3.4) e fundamentalistas (3.2). Provaremos abaixo que $Q_{S}$, definida em (3.4), é diferenciável em $w_{t}, w_{t}=\left(H_{t}, \bar{S}_{t}, \bar{F}_{t}\right)$ $(t=0,1,2, \ldots)$, desde que $\left(H_{0}+\lambda \bar{A}_{0}, \bar{A}_{0}\right) \neq(0,0)$, onde $\bar{A}_{0}=\alpha \bar{S}_{0}+(1-\alpha) \bar{F}_{0}$.

Lema 1. Seja $\left(w_{t}\right)_{t \geq 0}$ a seqüência definida em (2.20) para $\alpha>0$, e $Q_{F}$ e $Q_{S}$ dadas por (3.2) e (3.4), respectivamente. Se $\left(H_{0}+\lambda \bar{A}_{0}, \bar{A}_{0}\right) \neq(0,0)$, onde $\bar{A}_{0}=\alpha \bar{S}_{0}+(1-\alpha) \bar{F}_{0}$, então $Q_{S}$ é diferenciável em $w_{t}(t=0,1,2, \ldots)$.

Demonstração. Vamos supor inicialmente que, para $\left(w_{t}\right)_{t \geq 0}, w_{t}=\left(H_{t}, \bar{S}_{t}, \bar{F}_{t}\right)(t=$ $0,1,2, \ldots)$, como especificado no enunciado do lema, valha a seguinte implicação:

$$
\left(H_{0}+\lambda \bar{A}_{0}, \bar{A}_{0}\right) \neq(0,0) \Rightarrow\left(H_{t}+\lambda \bar{A}_{t}, \bar{A}_{t}\right) \neq(0,0), \quad \forall t=0,1,2, \ldots
$$

onde $\bar{A}_{t}=\alpha \bar{S}_{t}+(1-\alpha) \bar{F}_{t} \quad(t=0,1,2, \ldots)$.

O lema segue diretamente da implicação (3.5). Isto porque $Q_{S}$, definida em (3.4), é diferenciável em todos os pontos $w_{t}, t=0,1,2, \ldots$, que satisfazem o lado direito de (3.5). 
Vamos, portanto, mostrar agora que a implicação (3.5) é verdadeira. Por indução, basta provar

$$
\left(H_{0}+\lambda \bar{A}_{0}, \bar{A}_{0}\right) \neq(0,0) \Rightarrow\left(H_{1}+\lambda \bar{A}_{1}, \bar{A}_{1}\right) \neq(0,0)
$$

Demonstração de (3.6). Vamos supor inicialmente que $H_{0}+\lambda \bar{A}_{0} \neq 0$. Provaremos por contradição que $H_{1}+\lambda \bar{A}_{1} \neq 0$ ou $\bar{A}_{1} \neq 0$. Se $H_{1}+\lambda \bar{A}_{1}=0$ e $\bar{A}_{1}=0$, segue $H_{1}=0$. Mas, por hipótese, $H_{1}=H_{0}+\lambda \bar{A}_{0} \neq 0$ ! Por contradição, segue, portanto, $H_{1}+\lambda \bar{A}_{1} \neq 0$ ou $\bar{A}_{1} \neq 0$.

Vamos supor agora que $H_{0}+\lambda \bar{A}_{0}=0$ e $\bar{A}_{0} \neq 0$. Provaremos diretamente que $\bar{A}_{1} \neq 0$ :

$$
\left|\bar{A}_{t}\right|=\left|\alpha\left(2 Q_{S}\left(w_{0}\right)-1\right)+(1-\alpha) E_{F}\left(w_{0}\right)\right|=\left|\alpha\left(2 Q_{S}\left(w_{0}\right)-1\right)\right|=\alpha>0
$$

A primeira igualdade de (3.7) vem da definição do sistema dinâmico $\left(w_{t}\right)_{t \geq 0}$ em (2.20) e de $\alpha>0$ (hipótese do lema). A segunda igualdade de (3.7) vem de: $E_{F}\left(w_{0}\right)=0, \forall \alpha \in(0,1]$, pois, se $\alpha \in(0,1)$, então $E_{F}\left(w_{0}\right)=2 \Phi(0)-1=0$; e, se $\alpha=1$, então $E_{F} \equiv 0$. A terceira igualdade de (3.7) segue de $Q_{S}\left(w_{0}\right) \in\{0,1\}$, para $Q_{S}$ definido em (3.4) e $H_{0}+\lambda \bar{A}_{0}=0$. A desigualdade em (3.7) vem da hipótese do lema.

Devido à validade do Lema 1, podemos aplicar a Proposição 1 para as probabilidades de compra de fundamentalistas (3.2) e especuladores (3.4) e concluir a convergência em probabilidade de $\left(W_{t}\right)_{t \geq 0}$ para $\left(w_{t}\right)_{t \geq 0}$, para $w_{0}=\left(H_{0}, \bar{S}_{0}, \bar{F}_{0}\right)$ satisfazendo a condição inicial $\left(H_{0}+\lambda \bar{A}_{0}, \bar{A}_{0}\right) \neq(0,0)$, onde $\bar{A}_{0}=\alpha \bar{S}_{0}+(1-\alpha) \bar{F}_{0}$.

\subsubsection{Convergência de $\left(W_{t}\right)_{t \geq 0}$ para $\left(w_{t}\right)_{t \geq 0}$. Caso $J_{t}^{(s)}=0$ e $K_{t}^{(s)}>$} $0, s \in S, t \geq 0$

Provaremos agora a convergência de $\left(W_{t}\right)_{t \geq 0}$ para $\left(w_{t}\right)_{t \geq 0}$ no caso em que não há influência social de preço $\left(J_{t}^{(s)}=0, s \in S\right)$. Neste caso, levando em conta a nova regra de decisão especulativa dada em (3.1), a probabilidade de compra de especuladores $Q_{S}: \mathbb{R} \times[-1,1]^{2} \rightarrow[0,1]$ é dada como segue abaixo. Seja $w=(H, \bar{S}, \bar{F}) \in \mathbb{R} \times[-1,1]^{2}$, então

$$
Q_{S}(w)=\left\{\begin{array}{llll}
\Psi(\bar{S} /(H+\lambda \bar{A})) & \text { se } H+\lambda \bar{A}>0 & \\
1-\Psi(\bar{S} /(H+\lambda \bar{A})) & \text { se } H+\lambda \bar{A}<0 & \\
1 & \text { se } H+\lambda \bar{A}=0 \quad \text { e } \bar{S}>0 \\
0 & \text { se } H+\lambda \bar{A}=0 \text { e } \bar{S} \leq 0
\end{array}\right.
$$


onde $\bar{A}:=\alpha \bar{S}+(1-\alpha) \bar{F}$.

Como já havíamos explicado no início da Seção 3.2, para garantir a convergência em probabilidade de $\left(W_{t}\right)_{t \geq 0}$ para $\left(w_{t}\right)_{t \geq 0}$, basta garantir que $Q_{S}$, definida agora em (3.8), seja diferenciável em $w_{t}(t=0,1,2, \ldots)$ para $\alpha>0$, onde $\left(w_{t}\right)_{t \geq 0}$ é definido pelo sistema dinâmico (2.20) para as probabilidades de compra de especuladores (3.8) e fundamentalistas (3.2). Provaremos abaixo que $Q_{S}$, definida em (3.8), é diferenciável em $w_{t}, w_{t}=\left(H_{t}, \bar{S}_{t}, \bar{F}_{t}\right)(t=0,1,2, \ldots)$, desde que $\lambda$ seja suficientemente pequeno e para que a condição inicial $w_{0}=\left(H_{0}, \bar{S}_{0}, \bar{F}_{0}\right)$ valha $\left(H_{0}+\lambda \bar{A}_{0}, \bar{A}_{0}\right) \neq(0,0)$, onde $\bar{A}_{0}=\alpha \bar{S}_{0}+(1-\alpha) \bar{F}_{0}$.

Lema 2. Seja $\left(w_{t}\right)_{t \geq 0}$ a seqüência definida em (2.20) para $Q_{F}$ e $Q_{S}$ definidas em (3.2) $e$ (3.8), respectivamente, onde $0<\alpha \leq 1$ e $0 \leq \lambda<\lambda_{c}:=\left[(1-\alpha) 2 \Phi^{\prime}(0)\right]^{-1}$, caso $0<\alpha<1$. Se $\left(H_{0}+\lambda \bar{A}_{0}, \bar{S}_{0}\right) \neq(0,0)$, onde $\bar{A}_{0}=\alpha \bar{S}_{0}+(1-\alpha) \bar{F}_{0}$, então $Q_{S}$ é diferenciável em $w_{t}$ $(t=0,1,2, \ldots)$.

Demonstração. Vamos supor inicialmente que, para $\left(w_{t}\right)_{t \geq 0}, w_{t}=\left(H_{t}, \bar{S}_{t}, \bar{F}_{t}\right)(t=$ $0,1,2, \ldots)$, como especificado no enunciado do lema, valha a seguinte implicação:

$$
\left(H_{0}+\lambda \bar{A}_{0}, \bar{S}_{0}\right) \neq(0,0) \Rightarrow\left(H_{t}+\lambda \bar{A}_{t}, \bar{S}_{t}\right) \neq(0,0), \quad \forall t=0,1,2, \ldots
$$

onde $\bar{A}_{t}=\alpha \bar{S}_{t}+(1-\alpha) \bar{F}_{t} \quad(t=0,1,2, \ldots)$.

O lema segue diretamente da implicação (3.9). Isto porque $Q_{S}$, definida em (3.8), é diferenciável em todos os pontos $w_{t}, t=0,1,2, \ldots$, que satisfazem o lado direito de (3.9). Vamos, portanto, mostrar agora que a implicação (3.9) é verdadeira. Por indução, basta provar

$$
\left(H_{0}+\lambda \bar{A}_{0}, \bar{S}_{0}\right) \neq(0,0) \Rightarrow\left(H_{1}+\lambda \bar{A}_{1}, \bar{S}_{1}\right) \neq(0,0)
$$

Demonstração de (3.10). Vamos supor inicialmente que $H_{0}+\lambda \bar{A}_{0} \neq 0$. Provaremos por contradição que: ou $H_{1}+\lambda \bar{A}_{1} \neq 0$ ou $\bar{S}_{1} \neq 0$. Se ao contrário valesse $H_{1}+\lambda \bar{A}_{1}=0 \mathrm{e}$ $\bar{S}_{1}=0$, teríamos $\bar{A}_{1}=\alpha \bar{S}_{1}+(1-\alpha) \bar{F}_{1}=(1-\alpha) \bar{F}_{1}=(1-\alpha)\left[2 \Phi\left(-H_{1}\right)-1\right]$. Considerando que $2 \Phi-1$ é uma função ímpar, segue da igualdade acima e da condição $H_{1}+\lambda \bar{A}_{1}=0$ :

$$
H_{1}=\lambda(1-\alpha) 2\left[\Phi\left(H_{1}\right)-1\right]
$$


Agora, se $\alpha=1$, segue $H_{1}=0$. Mas, por hipótese, $H_{1}=H_{0}+\lambda \bar{A}_{0} \neq 0$ ! Por contradição, segue, portanto: ou $H_{1}+\lambda \bar{A}_{1} \neq 0$, ou $\bar{S}_{1} \neq 0$. Vamos supor agora: $0<\alpha<1$. Pelas propriedades de $\Phi$ em (2.5), a equação (3.11) possui soluções diferentes de zero em $H_{1}$ se, e somente se, $\lambda(1-\alpha) 2 \Phi^{\prime}(0)>1$. Como $\lambda<\lambda_{c}:=\left[(1-\alpha) 2 \Phi^{\prime}(0)\right]^{-1}$, a única solução de (3.11) é $H_{1}=0$. Mas, por hipótese, $H_{1}=H_{0}+\lambda \bar{A}_{0} \neq 0$. Portanto, concluímos novamente por contradição: ou $H_{1}+\lambda \bar{A}_{1} \neq 0$, ou $\bar{S}_{1} \neq 0$.

Vamos supor agora: $H_{0}+\lambda \bar{A}_{0}=0$ e $\bar{S}_{0} \neq 0$. Mostraremos que $\bar{S}_{1} \neq 0$. De acordo com a probabilidade de compra de especuladores $Q_{S}$, dada em (3.8), temos

$$
\left|\bar{S}_{1}\right|=\left|2 Q_{S}\left(w_{0}\right)-1\right|=1 \text {. }
$$

A primeira igualdade de (3.12) vem da definição do sistema dinâmico $\left(w_{t}\right)_{t \geq 0}$ em $(2.20)$ e de $\alpha>0$ (hipótese do lema). A segunda igualdade de (3.12) segue de $Q_{S}\left(w_{0}\right) \in\{0,1\}$ para $Q_{S}$ definido em (3.8) e $H_{0}+\lambda \bar{A}_{0}=0$.

Devido à validade do Lema 2, podemos aplicar a Proposição 1 para as probabilidades de compra de fundamentalistas (3.2) e especuladores (3.8) e concluir a convergência em probabilidade de $\left(W_{t}\right)_{t \geq 0}$ para $\left(w_{t}\right)_{t \geq 0}$, para $\lambda$ suficientemente pequeno e $w_{0}=\left(H_{0}, \bar{S}_{0}, \bar{F}_{0}\right)$ satisfazendo a condição inicial $\left(H_{0}+\lambda \bar{A}_{0}, \bar{S}_{0}\right) \neq(0,0)$, onde $\bar{A}_{0}=\alpha \bar{S}_{0}+(1-\alpha) \bar{F}_{0}$.

\subsection{Caso $J_{t}^{(s)}>0, K_{t}^{(s)}=0, \forall s \in S, \forall t \geq 0$}

Nesta seção analisaremos a dinâmica de $\left(w_{t}\right)_{t \geq 0}$ para o caso em que não há influência social direta. Analisaremos diretamente o caso em que $\lambda>0$ (onde $H_{t}$ varia no tempo).

\subsubsection{Dinâmica da sobrevalorização e excesso de demanda do ativo $\left(H_{t}, \bar{A}_{t}\right)_{t \geq 0}$}

A dinâmica para grandes populações $\left(w_{t}\right)_{t \geq 0}\left(w_{t}=\left(H_{t}, \bar{S}_{t}, \bar{F}_{t}\right), t=0,1,2, \ldots\right)$ está unicamente determinada pelo sistema dinâmico da sobrevalorização e excesso de demanda do ativo $\left(H_{t}, \bar{A}_{t}\right)_{t \geq 0}\left(\bar{A}_{t}=\alpha \bar{S}_{t}+(1-\alpha) \bar{F}_{t}, t=0,1,2, \ldots\right)$. Como nos casos anteriores, $\left(H_{t}, \bar{A}_{t}\right)_{t \geq 0}$ pode ser derivada das probabilidades de compra de especuladores $Q_{S}$ e fundamentalistas $Q_{F}$, agora dadas em (3.4) e (3.2), respectivamente. Para estas probabilidades de compra, a dinâmica de $\left(H_{t}, \bar{A}_{t}\right)_{t \geq 0}$ é dada por

$$
\left\{\begin{array}{l}
H_{t}=H_{t-1}+\lambda \bar{A}_{t-1} \\
\bar{A}_{t}=g\left(\bar{A}_{t-1} ; H_{t-1}+\lambda \bar{A}_{t-1}\right)
\end{array} \quad t=1,2,3 \ldots\right.
$$


onde $g$ é definida como segue abaixo:

$$
g(x ; h)= \begin{cases}+\alpha[2 \Psi(x / h)-1]+(1-\alpha)[2 \Phi(-h)-1] & \text { para } \quad h>0 \\ -\alpha[2 \Psi(x / h)-1]+(1-\alpha)[2 \Phi(-h)-1] & \text { para } h<0\end{cases}
$$

onde para $h=0$, temos $g(x ; h)=\alpha$ quando $x>0$, e $g(x ; h)=-\alpha$ quando $x \leq 0$.

Nosso objetivo agora é estudar o comportamento e os equilíbrios de $\left(H_{t}, \bar{A}_{t}\right)_{t \geq 0}$. Estes equilíbrios satisfazem $(H, \bar{A})=\digamma(H, \bar{A})$, onde, nesta seção, $\digamma: \mathbb{R} \times[-1,1] \rightarrow \mathbb{R} \times[-1,1]$, $\digamma(H, \bar{A})=(H+\lambda \bar{A}, g(\bar{A} ; H+\lambda \bar{A}))$, onde $g$ é definida por (3.14). Equilíbrios de (3.13) serão diferenciados em equilíbrios globalmente estáveis, localmente estáveis e instáveis, da mesma forma como os equilíbrios do sistema $\left(H_{t}, \bar{A}_{t}\right)_{t \geq 0}$, dados pela interação (2.81), foram classificados nestas categorias na Seção 2.4.2.2.

Características particulares do modelo. Observamos as seguintes diferenças que emergem neste modelo. Inicialmente observemos que, para $g$ apresentada em (3.14), temos

$$
g^{\prime}(0, h):=\left.\frac{\partial g(x ; h)}{\partial x}\right|_{x=0}=2 \alpha \Psi^{\prime}(0) / h
$$

Note que um deslocamento de $h$ não somente translada o gráfico de $g(\cdot ; h)$, como também altera a sua forma. Em particular, para $g^{\prime}(0, h)$ definida em $(3.15)$, temos $\lim _{h \rightarrow \pm 0} \partial g^{\prime}(0 ; h)=$ $\pm \infty$ e $\lim _{h \rightarrow \infty} g^{\prime}(0 ; h)=0$. Note também que, para qualquer $\bar{A} \in(0,+1], \lim _{h \rightarrow+0} g(\bar{A} ; h)=$ $\alpha$ e $\lim _{h \rightarrow \infty} g(\bar{A} ; h)=-1$. Estas propriedades fazem com que este modelo apresente um outro tipo de comportamento em comparação com o modelo apresentado no capítulo anterior (veja a Figura 3.1).

Notamos que neste modelo a sobrevalorização crítica do ativo $h_{*}$ está definida para qualquer $\alpha$ positivo. Em outras palavras, neste caso $\left(J_{t}^{(s)}>0, K_{t}^{(s)}=0, \forall s \in S\right)$, o modelo aponta para a formação de bolhas especulativas e crashes de preço do ativo para qualquer fração positiva de especuladores. Em analogia ao modelo de Ising ferromagnético, podemos dizer que, em certo sentido, a força de interação entre os spins (especuladores), 1/ $h$, aparece na definição da função $g$ em (3.14) inversamente proporcional ao valor negativo do "campo externo" - $h$ (subvalorização do ativo). Quando o "campo externo" é pequeno em valor absoluto, o valor absoluto da força de interação entre os spins $|1 / h|$, torna-se suficientemente grande, a ponto de o sistema com $H_{t}=h$ constante apresentar transição 


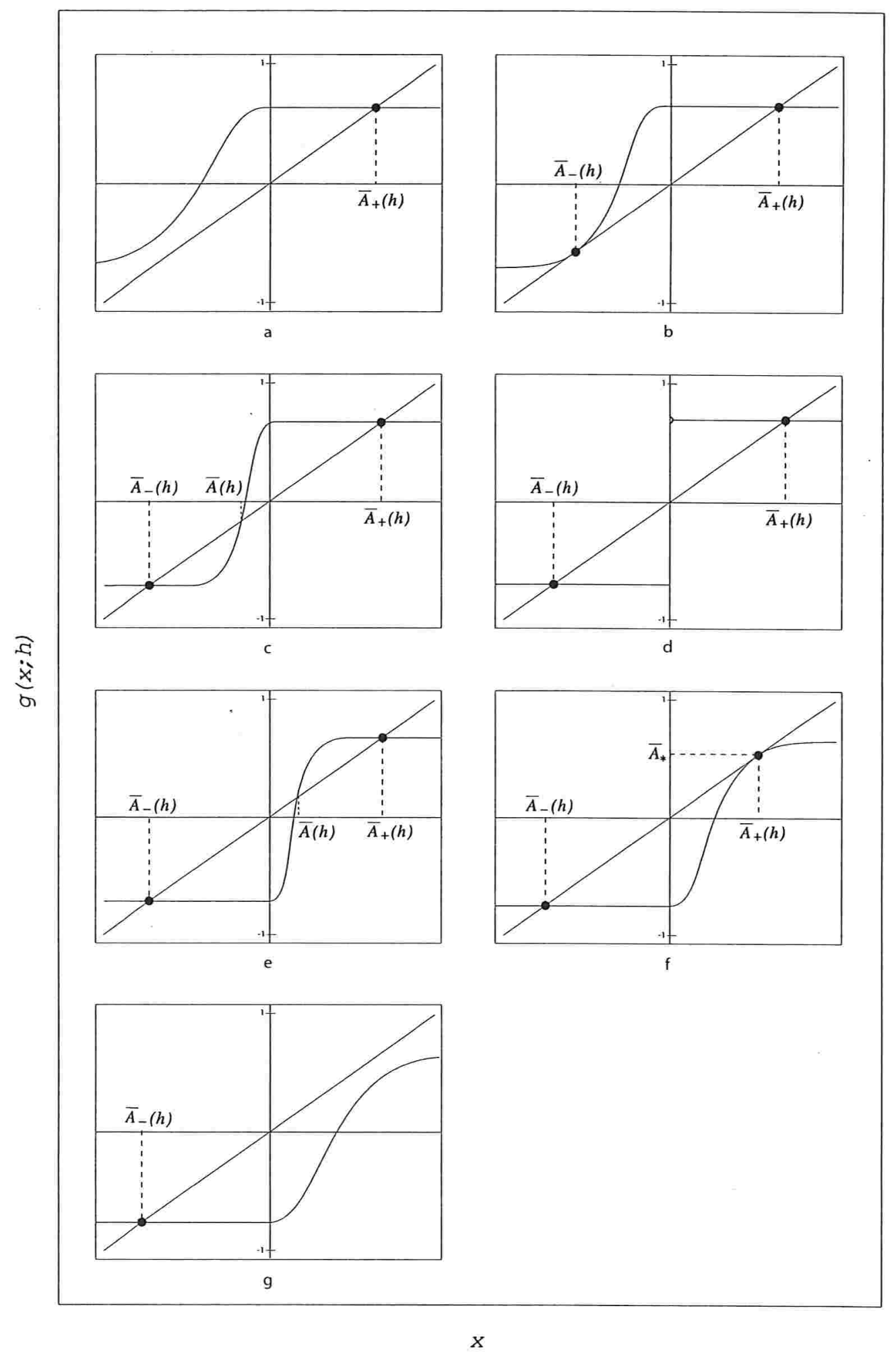

Figura 3.1: Gráfico de $g(\cdot ; h)$ definida em (3.14): a) $h<-h_{*} ;$ b) $h=-h_{*} ;$ c) $-h_{*}<h<0$; d) $h=0$; e) $0<h<h_{*}$; f) $h=-h_{*}$; e g) $h>h_{*}$. 
de fase. Vale observar que neste modelo, quando $\alpha>0$, a sobrevalorização crítica do ativo $h_{*}\left(=h_{*}(\alpha, \Phi, \Psi)\right)$ está sempre definida para quaisquer funções de distribuição de probabilidade $\Phi$ e $\Psi$, que satisfazem as propriedades apresentadas em (2.5) e as propriedades apresentadas na Seção 3.1.2, respectivamente. Ou seja, dados $\alpha>0$ e $\Phi$ e $\Psi$ satisfazendo as propriedades acima mencionadas, o sistema com $H_{t}=h$ constante apresenta transição de fase sempre que $|h|<h_{*}=h_{*}(\alpha, \Phi, \Psi)$. Lembramos que este não é o caso do modelo apresentado no capítulo anterior. Naquele modelo, $\left(\Phi^{\prime}(0)\right)^{-1}$ (dispersão das variáveis $P_{t}^{(i)}$, $i \in A, t \geq 0$ ), era determinante para a ocorrência de transição de fase.

\subsubsection{O resultado principal: comportamento cíclico de $\left(H_{t}, \bar{A}_{t}\right)_{t \geq 0}$}

Diferentemente do que ocorre no caso correspondente do modelo anterior $(J>0, K=0)$, o sistema dinâmico $\left(H_{t}, \bar{A}_{t}\right)$ é sempre atraído para ciclos, correspondendo à formação de bolhas especulativas e crashes de preço do ativo sempre que $\alpha>0$. Somente quando $\alpha=$ $0,\left(H_{t}, \bar{A}_{t}\right)$ possui um único equilíbrio globalmente estável em $(0,0)$. Mais especificamente, temos:

Proposição 7. Seja $\left(H_{t}, \bar{A}_{t}\right)_{t \geq 0}$ dado por (3.13). Existe um número positivo $\lambda_{c}$, tal que, para todo $\lambda$ com $0<\lambda<\lambda_{c}$, vale:

1. se $\alpha=0$

então $(0,0)$ é um equilíbrio globalmente estável de $\left(H_{t}, \bar{A}_{t}\right)_{t \geq 0}$;

2. se $0<\alpha \leq 1$

então $\left(H_{t}, \bar{A}_{t}\right)_{t \geq 0}$ não possui equilibrios e vale:

(a) $|\mathcal{T}|=\infty$;

(b) $\exists \tau_{\lambda} \in \mathbb{N}:\left|H_{\tau}\right|>h_{*} \forall \tau \in \mathcal{T} \operatorname{com} \tau>\tau_{\lambda}$,

onde $\mathcal{T}=\left\{\tau \in \mathbb{N} \mid \bar{A}_{\tau} \cdot \bar{A}_{\tau+1} \leq 0\right\}$ denota o conjunto dos tempos de reversão de sinal de $\left(\bar{A}_{t}\right)_{t \geq 0}$ e $h_{*}$ é a primeira componente da solução $\left(h_{*}, \bar{A}_{*}\right) \in \mathbb{R}_{+}^{2}$ do sistema de equações

$$
\bar{A}_{*}=g\left(\bar{A}_{*}, h_{*}\right) ; \quad \partial g\left(\bar{A}_{*}, h_{*}\right) / \partial \bar{A}_{*}=1
$$

onde g é definida por (3.14). 
A prova da Proposição 7 pode ser construída de maneira análoga à prova da Proposição 4. Uma idéia intuitiva desta prova pode ser desenvolvida de maneira análoga à idéia intuitiva da prova da Proposição 4 apresentada na Seção 2.4.2.3. Omitiremos a prova da Proposição 7. Não trataremos os melhoramentos da convergência em probabilidade do processo $\left(W_{t}\right)_{t \geq 0}$ para o processo $\left(w_{t}\right)_{t \geq 0}$.

Observação. Lembramos que este modelo aponta formalmente para a formação de bolhas especulativas e crashes do preço do ativo, qualquer que seja a fração de especuladores $\alpha>0$. Somente no caso de ausência de especuladores, o preço $P_{t}$ converge para o valor fundamental do ativo $\bar{P}$. Este fato pode causar um certo estranhamento ao leitor. A título de esclarecimento, observamos que, apesar da sobrevalorização crítica do ativo, $h_{*}>0$, estar sempre definida para qualquer que seja $\alpha>0$, temos também que $h_{*}$ é uma função crescente de $\alpha$, i.e., $\partial h_{*} / \partial \alpha \geq 0$, onde vale também $\lim _{\alpha \rightarrow 0} h_{*}(\alpha)=0$. Observamos ainda que esta monotonicidade de $h_{*}(\alpha)$ em relação a $\alpha$ pode ser verificada também no modelo apresentado no capítulo anterior. Ou seja, se $\alpha$ for positivo, mas muito pequeno, o preço do ativo $P_{t}$ não converge para $\bar{P}$; no entanto, $P_{t}$ será atraído para uma região próxima a $\bar{P}$, onde $P_{t}$ apresenta apenas uma pequena oscilação (crashes de amplitude próxima a $2 h_{*}$, relativamente pequena) em torno de $\bar{P}$. 


\subsection{Caso $J_{t}^{(s)}=0, K_{t}^{(s)}>0, \forall s \in S, \forall t \geq 0$}

Nesta seção analisaremos a dinâmica de $\left(w_{t}\right)_{t \geq 0}$ para o caso em que não há influência social de preço. Analisaremos diretamente o caso em que $\lambda>0$ (onde $H_{t}$ varia no tempo).

\subsubsection{Dinâmica da sobrevalorização e excesso de demanda do ativo $\left(H_{t}, \bar{A}_{t}\right)_{t \geq 0}$}

A dinâmica para grandes populações $\left(w_{t}\right)_{t \geq 0}\left(w_{t}=\left(H_{t}, \bar{S}_{t}, \bar{F}_{t}\right), t=0,1,2, \ldots\right)$ está unicamente determinada pelo sistema dinâmico da sobrevalorização e excesso de demanda do ativo $\left(H_{t}, \bar{A}_{t}\right)_{t \geq 0}\left(\bar{A}_{t}=\alpha \bar{S}_{t}+(1-\alpha) \bar{F}_{t}, t=0,1,2, \ldots\right)$. Como nos casos anteriores, $\left(H_{t}, \bar{A}_{t}\right)_{t \geq 0}$ pode ser derivada das probabilidades de compra de especuladores $Q_{S}$ e fundamentalistas $Q_{F}$, agora dadas em (3.8) e (3.2), respectivamente. Para estas probabilidades de compra, a dinâmica de $\left(H_{t}, \bar{A}_{t}\right)_{t \geq 0}$ é dada por

$$
\left\{\begin{array}{l}
H_{t}=H_{t-1}+\lambda \bar{A}_{t-1} \\
\bar{A}_{t}=g\left(\bar{A}_{t-1} ; H_{t-1}+\lambda \bar{A}_{t-1}\right)
\end{array} \quad t=1,2,3 \ldots\right.
$$

onde $g$ é dada para $\alpha>0$ e $h \neq 0$ por

$g(x ; h):=\left\{\begin{array}{l}+\alpha F_{2}\left(\left[x-(1-\alpha) F_{1}(-h)\right] /(\alpha h)\right)+(1-\alpha) F_{1}(-h) \text { para } \quad h>0 \\ -\alpha F_{2}\left(\left[x-(1-\alpha) F_{1}(-h)\right] /(\alpha h)\right)+(1-\alpha) F_{1}(-h) \text { para } h<0\end{array}\right.$

Para $\alpha=0, g(x ; h):=F(-h)$ e $h=0, g(x ; h):=\alpha$ quando $x>0$ e $g(x ; h)=-\alpha$ quando $x \leq 0$; onde $F_{1}:=2 \Phi-1$ e $F_{2}:=2 \Psi-1$.

Nosso objetivo agora é estudar o comportamento e os equilíbrios de $\left(H_{t}, \bar{A}_{t}\right)_{t \geq 0}$. Estes equilíbrios satisfazem $(H, \bar{A})=\digamma(H, \bar{A})$, onde, nesta seção, $\digamma: \mathbb{R} \times[-1,1] \rightarrow \mathbb{R} \times[-1,1]$, $\digamma(H, \bar{A})=(H+\lambda \bar{A}, g(\bar{A} ; H+\lambda \bar{A}))$, onde $g$ é definida por (3.18). Equilíbrios de (3.17) serão diferenciados em equilíbrios globalmente estáveis, localmente estáveis e instáveis, da mesma forma como os equilíbrios do sistema $\left(H_{t}, \bar{A}_{t}\right)_{t \geq 0}$, dado pela interação (2.81), foram classificados nestas categorias na Seção 2.4.2.2. 


\subsubsection{O resultado principal: transição de fase entre existência de dois equilíbrios estáveis de $\left(H_{t}, \bar{A}_{t}\right)_{t \geq 0}$ e comportamento cíclico de $\left(H_{t}, \bar{A}_{t}\right)_{t \geq 0}$}

Diferentemente do modelo anterior, neste modelo somente a fração de especuladores é determinante para a existência ou não de mais de um preço localmente estável do ativo.

Obtemos aqui os seguintes resultados: quando não há especuladores no mercado, $\alpha=0$, o sistema $\left(H_{t}, \bar{A}_{t}\right)_{t \geq 0}$ é atraído para $(0,0)$, i.e., $\left(H_{t}, \bar{A}_{t}\right)_{t \geq 0}$ possui um único equilíbrio globalmente estável em $(0,0)$. Diferentemente do modelo anterior, $(0,0)$ não é um equilíbrio do sistema $\left(H_{t}, \bar{A}_{t}\right)_{t \geq 0}(\digamma(0,0)=(0,-\alpha) \neq(0,0))$ para qualquer $\alpha>0$. Se a fração de especuladores for positiva, porém suficientemente pequena, então dois outros equilíbrios localmente estáveis surgirão; se, no entanto, a fração de especuladores for suficientemente grande, o sistema $\left(H_{t}, \bar{A}_{t}\right)_{t \geq 0}$ será atraído para um ciclo correspondendo à formação de bolhas especulativas e crashes de preço do ativo no sentido já descrito na Proposição 7. Dependendo somente da fração $\alpha$ de especuladores no mercado, teremos um ou outro cenário. Mais especificamente, temos:

Proposição 8. Seja $\left(H_{t}, \bar{A}_{t}\right)_{t \geq 0}$ dado por (3.17). Para $\alpha>0$, seja $\left(h_{*}^{(\alpha)}, \bar{A}_{*}^{(\alpha)}\right)$ a única solução de componentes positivas do sistema de equações

$$
\bar{A}_{*}^{(\alpha)}=g\left(\bar{A}_{*}^{(\alpha)}, h_{*}^{(\alpha)}\right) ; \quad \partial g\left(\bar{A}_{*}^{(\alpha)}, h_{*}^{(\alpha)}\right) / \partial \bar{A}_{*}^{(\alpha)}=1
$$

onde g é dada por (3.18). Seja $\alpha_{*}>0$ dada por

$$
\alpha_{*}:=\frac{\left[2 \Phi\left(h_{*}^{(1)}\right)-1\right]}{\bar{A}_{*}^{(1)}+\left[2 \Phi\left(h_{*}^{(1)}\right)-1\right]} \quad(<1)
$$

onde $h_{*}^{(1)}$ e $\bar{A}_{*}^{(1)}$ são determinados por (3.19) para $\alpha=1$.

Existe $\lambda_{c}>0$, tal que, para todo $\lambda$ com $0<\lambda<\lambda_{c}$, vale:

1. Se $\alpha=0$

então $(0,0)$ é o único equilíbrio globalmente estável de $\left(H_{t}, \bar{A}_{t}\right)$;

2. Se $0<\alpha \leq \alpha_{*}$

então $\left(H_{t}, \bar{A}_{t}\right)$ possui dois equilibrios, denotados por $E_{+}$e $E_{-}$, onde $E_{-}=-E_{+}$, $E_{+}:=\left(\bar{H}_{+}, 0\right)$ para um certo $\bar{H}_{+}$, que satisfaz $0<\bar{H}_{+}<h_{*}^{(1)}$, e onde $h_{*}^{(1)}$ é determinado por (3.19) para $\alpha=1$. Para estes equilibrios, vale: 
(a) Se $0<\alpha<\alpha^{*}$

então $E_{+}$e $E_{-}$são localmente estáveis, onde

$\left\{(h, 0) \mid h<-h_{*}^{(1)}\right.$ ou $\left.0<h \leq h_{*}^{(1)}\right\} \quad$ e $\quad\left\{(h, 0) \mid-h_{*}^{(1)}<h<0\right.$ ou $\left.h>h_{*}^{(1)}\right\}$

pertencem ao domínio de atração de $E_{+}$e $E_{-}$, respectivamente;

(b) Se $\alpha=\alpha^{*}$

então $E_{+}$e $E_{-}$são instáveis;

3. Se $\alpha^{*}<\alpha \leq 1$

então $\left(H_{t}, \bar{S}_{t}, \bar{A}_{t}\right)_{t \geq 0}$ não possui equilíbrios. Além disso, vale:

(a) $|\mathcal{T}|=\infty$;

(b) $\exists \tau_{\lambda} \in \mathbb{N}:\left|H_{\tau}\right|>h_{*} \forall \tau \in \mathcal{T} \operatorname{com} \tau>\tau_{\lambda}$,

onde $\mathcal{T}=\left\{\tau \in \mathbb{N} \mid \bar{A}_{\tau} \cdot \bar{A}_{\tau+1} \leq 0\right\}$ denota o conjunto dos tempos de reversão de sinal de $\left(\bar{A}_{t}\right)_{t \geq 0}$.

A prova da Proposição 8 é análoga à prova da Proposição 6. Omitiremos a sua prova neste trabalho. Não trataremos a questão do melhoramento da convergência de $\left(W_{t}\right)_{t \geq 0}$ para $\left(w_{t}\right)_{t \geq 0}$ neste caso.

Observações finais. Observamos que tanto no presente caso $\left(J_{t}^{(s)}=0, K_{t}^{(s)}>0, \forall s \in S\right.$, $\forall t \geq 0)$ do presente modelo como no caso análogo do modelo anterior $(J=0, K>0)$, obtemos três situações típicas:

1. $\left(H_{t}, \bar{A}_{t}\right)_{t \geq 0}$ possui um único equilíbrio globalmente estável em $(0,0)$ (unicidade do preço de equilíbrio de mercado em $\bar{P}$ );

2. $\left(H_{t}, \bar{A}_{t}\right)_{t \geq 0}$ possui dois equilíbrios localmente estáveis (existência de dois preços de equilíbrio de mercado: $\bar{P}_{-}:=\bar{P}-\bar{H}_{+}$e $\left.\bar{P}_{+}:=\bar{P}+\bar{H}_{+}\right)$; e

3. $\left(H_{t}, \bar{A}_{t}\right)_{t \geq 0}$ é atraído para ciclos (formação de bolhas especulativas e crashes de preço do ativo). 
A particularidade do presente modelo é que, no caso em que $J_{t}^{(s)}=0, K_{t}^{(s)}>0$, $\forall s \in S, \forall t \geq 0$, a ocorrência das situações 1), 2) e 3) depende somente da fração $\alpha$ de especuladores, i.e., situação 1) se $\alpha=0$; situação 2) se $0<\alpha \leq \alpha_{*}$; e situação 3) se $\alpha_{*}<\alpha \leq 1$. Lembramos que no caso análogo do modelo anterior $(J=0, K>0)$, as situações 2) e 3) ocorrem somente quando $\left(\Phi^{\prime}(0)\right)^{-1}$ é suficientemente pequeno. 


\section{Capítulo 4}

\section{Modelo de Influência Social Determinística com Interação Local entre Especuladores}

\subsection{Definição do modelo}

No modelo apresentado no Capítulo 2 introduzimos o parâmetro $K>0$ (influência social direta). Com esse parâmetro, havíamos modelado a tendência de especuladores alinharem suas atitudes de investimento à atitude média de investimento dos especuladores. Esta componente do modelo visou modelar o contágio (direto) de opiniões entre especuladores. No entanto, na vida real, este contágio ocorre pelas relações de cada especulador com o seu grupo de referência (seus vizinhos mais próximos). Com o modelo apresentado no Capítulo 2 aproximamos, portanto, o efeito macroscópico destas relações, a fim de investigar algo que possa ocorrer sob contágio local de opiniões.

Vamos investigar agora se os fenômenos que derivamos no modelo apresentado no Capítulo 2 sobrevivem quando considerarmos uma rede de influências específica entre especuladores. Vamos definir inicialmente as componentes básicas do modelo.

\subsubsection{Atitude de investimento e excesso de demanda relativo em tempo contínuo}

A cada instate $t \in \mathbb{R}_{+}$, um conjunto de agentes, denotado por $A$, demanda ou oferta uma quantidade fixa $q$ do ativo. A atitude do agente $i \in A$ no tempo $t$ será denotada por 
$X_{t}^{(i)}$ seguindo a convenção

$$
X_{t}^{(i)}= \begin{cases}+1 & \text { agente } i \text { demanda uma quantidade } q \text { do ativo no tempo } t \\ -1 & \text { agente } i \text { oferece uma quantidade } q \text { do ativo no tempo } t\end{cases}
$$

onde novamente, para $A$ não vazio e finito, definimos o excesso de demanda relativo ou simplesmente "excesso de demanda" por

$$
\bar{A}_{t}=\sum_{i \in A} X_{t}^{(i)} / N \quad \forall t \in \mathbb{R}_{+}
$$

onde $N$ denota a cardinalidade de $A$.

\subsubsection{Ajustamento do preço em tempos exponenciais}

Como no modelo apresentado no Capítulo 2, incluímos neste modelo a figura de um market maker que, de tempos em tempos, ajusta o preço do ativo ao excesso de demanda observado e garante o preço ajustado até o próximo ajuste de preço. Denotaremos os tempos nos quais o market maker reage e ajusta o preço ao excesso de demanda observado por $t_{0}^{(m)}, t_{1}^{(m)}, t_{2}^{(m)}, \ldots$ Estes tempos satisfazem:

1. $t_{0}^{(m)}=0$.

2. $t_{k+1}^{(m)} \geq t_{k}^{(m)}, \forall k=0,1,2, \ldots$

3. $t_{k+1}^{(m)}-t_{k}^{(m)} \forall k=0,1,2, \ldots$ são variáveis aleatórias independentes e identicamente distribuídas segundo uma distribuição exponencial de parâmetro $\xi$.

Introduzimos o preço ${ }^{1}$ no instante $t \in \mathbb{R}_{+}$, como segue:

$$
P_{t}=P_{t_{k}^{(m)}}+\lambda \bar{A}_{t_{k}^{(m)}}, \quad \text { onde } k \text { é tal que } \quad t \in\left(t_{k}^{(m)}, t_{k+1}^{(m)}\right]
$$

Observamos que, durante os intervalos de tempo $\left(t_{k}^{(m)}, t_{k+1}^{(m)}\right], k=0,1,2, \ldots$, o preço não se altera. Ou seja, o processo $\left(P_{t}\right)_{t \geq 0}$ é um processo de saltos em tempo contínuo. A cada instante aleatório $t_{k}^{(m)}, k=0,1,2, \ldots$, o preço é atualizado segundo o excesso de demanda corrente. Como veremos a seguir, observamos ainda que o excesso de demanda $\bar{A}_{t}$ não permanece constante durante os intervalos de tempo $\left(t_{k}^{(m)}, t_{k+1}^{(m)}\right], k=0,1,2, \ldots$

\footnotetext{
${ }^{1}$ Como no modelo apresentado no Capítulo 2, optamos por usar a notação " $P_{t}$ " para denotar o logaritmo do preço no instante $t$ e nos referir a $P_{t}$ com a expressão "preço" ou "preço do ativo".
} 


\subsubsection{Tempos exponenciais de atualização de atitude de inves- timento}

Postulamos que cada agente $i \in A$ (especulador ou fundamentalista) atualiza sua atitude de investimento somente em seus tempos de atualização de atitude de investimento $t_{1}^{(i)}, t_{2}^{(i)}, t_{3}^{(i)}, \ldots$ Nos demais tempos, cada agente $i \in A$ mantém a atitude atualizada até o próximo instante de atualização, i.e., para qualquer agente $i \in A$, vale

$$
X_{t}=X_{t_{k+1}^{(i)}} \quad \forall t \in\left(t_{k}^{(i)}, t_{k+1}^{(i)}\right], \quad k=0,1,2 \ldots
$$

onde, para os tempos de atualização de atitude de investimento, vale:

1. $t_{0}^{(i)}=0, \forall i \in A$.

2. $t_{k+1}^{(i)} \geq t_{k}^{(i)}, \forall k=0,1,2, \ldots, \forall i \in A$.

3. $t_{k+1}^{(i)}-t_{k}^{(i)}, \forall k=0,1,2, \ldots, \forall i \in A$, são variáveis aleatórias independentes e identicamente distribuídas segundo uma distribuição exponencial de parâmetro 1 .

4. $t_{k+1}^{(i)}-t_{k}^{(i)}, \forall k=0,1,2, \ldots, \forall i \in A \cup\{m\}$, são variáveis aleatórias independentes.

Mais adiante, esclareceremos a função do parâmetro $\xi$, que apresentamos no item 3 da seção anterior (parâmetro da exponencial de atualização do preço, podendo valer $\xi \neq 1$ ).

\subsubsection{Heterogeneidade das avaliações individuais sobre o valor fundamental do ativo (forma paramétrica de $\Phi$ )}

Como no modelo apresentado no Capítulo 2, postularemos que, no instante de atualização de atitude de investimento $t_{k}^{(i)}$, o agente $i$ forme uma avaliação individual sobre o valor fundamental do ativo denotada por $P_{t_{k}^{(i)}}^{(i)}(i \in A, k=0,1,2, \ldots)$. Mediante esta avaliação, entre outros fatores, o agente $i$ atualiza sua atitude de investimento no instante $t_{k}^{(i)}(i \in A$, $k=0,1,2, \ldots)$. Para $P_{t_{k}^{(i)}}^{(i)}(i \in A, k=0,1,2, \ldots)$, vale

$$
P_{t_{k}^{(i)}}^{(i)}=\bar{P}+\theta_{t_{k}^{(i)}}^{(i)} \quad\left(\theta_{t_{k}^{(i)}}^{(i)} \sim \Phi\right) \quad i \in A, k=0,1,2 \ldots
$$

Analogamente ao modelo apresentado no Capítulo 2, postularemos que as divergências entre as avaliações individuais e a avaliação média, expressas pelas variáveis $\theta_{t_{k}^{(i)}}^{(i)}, i \in$ 
$A, k=0,1,2, \ldots$, são variáveis aleatórias independentes e identicamente distribuídas mediante uma função de distribuição de probabilidades acumulada $\Phi$. Neste modelo, a função $\Phi$ possui a seguinte forma paramétrica:

$$
\Phi(x)=\frac{\exp (2 \beta x)}{1+\exp (2 \beta x)} \quad \forall x \in \mathbb{R}
$$

onde $\beta$ é um parâmetro positivo.

Observamos que $\Phi$ possui as mesmas propriedades apresentadas em (2.5), das quais partimos no modelo do Capítulo 2, i.e., para a derivada $\Phi^{\prime}$ (função de densidade de probabilidade de $\left.\theta_{t_{k}^{(i)}}^{(i)}\right)$, vale:

1) Simetria: $\quad \Phi^{\prime}(x)=\Phi^{\prime}(|x|) \quad \forall x \in \mathbb{R} \quad$ e

2) Monotonicidade: $\Phi^{\prime}$ é estritamente decrescente em $[0, \infty)$

Continuaremos adotando $\left(\Phi^{\prime}(0)\right)^{-1}$ como uma medida de heterogeneidade das avaliações individuais sobre o valor fundamental do ativo. Neste caso paramétrico, segue

$$
\left(\Phi^{\prime}(0)\right)^{-1}=2 / \beta
$$

Ou seja, quanto menor for $\beta$, maior será a heterogeneidade (dispersão) das avaliações individuais sobre o valor fundamental do ativo.

\subsubsection{Fundamentalistas, especuladores e a rede de influências}

Como no modelo apresentado no Capítulo 2, o conjunto $A$ de todos os agentes consiste de dois subconjuntos: um subconjunto de especuladores $S$ e um subconjunto de fundamentalistas $F(A=S \cup F)$, atuando em uma proporção $\alpha \in[0,1]$ no mercado, i.e.,

$$
N_{S} / N=\alpha, \quad N_{F} / N=(1-\alpha)
$$

onde $N_{S}$ e $N_{F}$ denotam o número de especuladores e de fundamentalistas, respectivamente.

Definimos o conjunto de fundamentalistas como sendo um conjunto enumerável, digamos $F=\left\{1,2, \ldots N_{F}\right\}$; e o conjunto de especuladores como sendo uma caixa quadrada em $\mathbb{Z}^{d}$, ou seja:

$$
S:=\left[-N_{S}^{\prime},-\left(N_{S}^{\prime}-1\right), \ldots,-2,-1,0,+1,+2,+\left(N_{S}^{\prime}-1\right),+N_{S}^{\prime}\right]^{d}
$$


onde $N_{S}=\left(2 N_{S}^{\prime}+1\right)^{d}$ (número de especuladores).

Vamos definir agora a rede de influências entre especuladores. Definiremos os "vizinhos mais próximos de $s^{\prime \prime}(s \in S)$ (que influenciam a opinião de $s$ diretamente) pelo conjunto

$$
V^{(s)}:=\{v \in S:\|s-v\|=1\}
$$

onde $\|\cdot\|$ denota a norma euclidiana em $\mathbb{R}^{2}$. Denotaremos por $\bar{S}_{t}^{(s)}$ a média aritmética das atitudes de investimento dos vizinhos mais próximos de $s$ no tempo $t \in \mathbb{R}_{+}$, ou seja:

$$
\bar{S}_{t}^{(s)}:=\frac{1}{2 d} \sum_{v \in V^{(s)}} X_{t}^{(v)}
$$

\subsubsection{Regra de decisão fundamentalista}

A regra de decisão de um fundamentalista $f \in F$ permanece essencialmente a mesma apresentada em (2.9). A única diferença está nos tempos de atualização, i.e., para $t \in \mathbb{R}_{+}$, vale

$$
X_{t}^{(f)}=\left\{\begin{array}{ccc}
+1 & \text { se } & -\left(P_{t_{k}^{(f)}}-P_{t_{k}^{(f)}}^{(f)}\right)>0 \\
-1 & \text { caso contrário }
\end{array}\right.
$$

onde $k$ é tal que $t \in\left(t_{k}^{(f)}, t_{k+1}^{(f)}\right]$.

\subsubsection{Regra de decisão especulativa sob influência social local}

Apresentaremos agora a regra de decisão especulativa. Com esta regra, modelamos a tendência de cada especulador $s \in S$ ser influenciado diretamente pela opinião de seu grupo de referência, i.e., seus "vizinhos mais próximos". Para o especulador $s \in S$ no tempo $t$, postulamos

$$
X_{t}^{(s)}=\left\{\begin{array}{lll}
+1 & \text { se } & J \bar{A}_{t_{k}^{(s)}}+K \bar{S}_{t_{k}^{(s)}}^{(s)}-\left(P_{t_{k}^{(s)}}-P_{t_{k}^{(s)}}^{(s)}\right)>0 \\
-1 & \text { caso contrário }
\end{array}\right.
$$

onde $k$ é tal que $t \in\left(t_{k}^{(s)}, t_{k+1}^{(s)}\right]$ e onde $J \geq 0$ e $K \geq 0$.

Observamos que a regra de decisão acima é análoga à que apresentamos em (2.12). A diferença essencial é que agora o especulador $s$ é influenciado diretamente apenas pela média aritmética das atitudes de seus vizinhos mais próximos. 


\subsubsection{Condição inicial}

Para fecharmos o modelo, resta apenas indicar a condição inicial no tempo $t=0$. Vamos considerar inicialmente $P_{0} \in \mathbb{R}$ e uma configuração de atitudes de investimento de especuladores igualmente arbitrária, i.e., $X_{0}^{(s)} \in\{-1,1\}, \forall s \in S$.

\subsection{Processo da sobrevalorização do ativo, configu- ração de atitudes de investimento de especula- dores e média de fundamentalistas}

Nosso objetivo final é obter informações sobre o comportamento do processo estocástico $\left(P_{t}\right)_{t \geq 0}$. Vamos reduzir o processo definido nas seções anteriores de forma a facilitar este estudo.

Analogamente ao modelo apresentado no Capítulo 2, definiremos a sobrevalorização $H_{t}$ do ativo pela diferença entre preço e avaliação média sobre o valor fundamental do ativo no tempo $t \in \mathbb{R}_{+}$, i.e., $H_{t}:=P_{t}-\bar{P}, \quad \forall t \in \mathbb{R}_{+}$. Diferentemente do que ocorre no modelo apresentado no Capítulo 2 , o processo $\left(W_{t}\right)_{t \geq 0}$, onde $W_{t}=\left(H_{t}, \bar{S}_{t}, \bar{F}_{t}\right)$ com $\bar{S}_{t}=\sum_{s \in S} X_{t}^{s} / N$ e $\bar{F}_{t}=\sum_{f \in F} X_{t}^{f} / N$, não é mais Markoviano. Isto porque a atitude de investimento de cada especulador $s \in S$ depende agora das atitudes de investimento de seus vizinhos mais próximos, e não da média das atitudes de todos os especuladores. Vamos considerar, portanto, o seguinte processo Markoviano:

$$
\left(W_{t}\right)_{t \geq 0} \quad \text { onde } W_{t}=\left(H_{t}, X_{t}, \bar{F}_{t}\right) \quad t \in \mathbb{R}_{+}
$$

onde $X_{t}$ denota a configuração das atitudes de investimento de todos os especuladores no tempo $t$, i.e., onde

$$
X_{t} \in\{-1,1\}^{S}, \forall t \in \mathbb{R}_{+}, \quad \text { onde } X_{t}(s)=X_{t}^{(s)}, \forall s \in S, \forall t \in \mathbb{R}_{+}
$$

Da definição (4.13) segue que $X_{t}^{(s)}$ corresponde à atitude de investimento do especulador $s$ dada a configuração $X_{t}$ e que $S_{t}^{(s)}$ corresponde à média aritmética das atitudes de investimento dos vizinhos mais próximos de $s$ dada a configuração $X_{t}$. 


\subsection{Processo da sobrevalorização e excesso de de- manda do ativo $\left(H_{t}, \bar{A}_{t}\right)_{t \geq 0}$}

Nas próximas seções estudaremos o comportamento do processo $\left(H_{t}, \bar{A}_{t}\right)_{t \geq 0}$ para uma grande população de agentes. Este processo será estudado a partir das propriedades do processo $\left(W_{t}\right)_{t \geq 0}$ definido em (4.12). Estudaremos o caso em que $\lambda=0$ ( $H_{t}=h$ constante) e o caso em que $\lambda>0$ ( $H_{t}$ varia no tempo). Trataremos somente o caso em que $J=0$, $K \geq 0$ (apenas influência social direta) e $d=2\left(S \subset \mathbb{Z}^{2}\right)$. Apresentaremos nesta seção as linhas gerais que nortearão este estudo.

Para $\lambda=0$, i.e., $H_{t}=h$ fixo, derivaremos inicialmente a medida invariante $\mu_{S}$ do processo $\left(X_{t}\right)_{t \geq 0}$ das configurações das atitudes de investimento de especuladores, para $S$ finito. Como veremos, a medida $\mu_{S}$ será a medida de Gibbs com a seguinte Hamiltoniana:

$$
\beta \sum_{s \in S} X^{(s)}\left[K \bar{S}^{(s)}-h\right]
$$

onde $X^{(s)}$ é o valor atribuído ao sítio $s \in S \subset \mathbb{Z}^{2}(-1$ ou +1$)$ e $\bar{S}^{(s)}$ é a média aritmética dos vizinhos mais próximos de $s \in S$, dada a configuração $X$.

Em seguida, para $h \neq 0$, ou $\beta$ suficientemente pequeno, ou $\alpha=0$ (campo externo diferente de zero, ou temperatura acima da temperatura crítica, ou ausência de especuladores), definiremos o excesso de demanda de equilíbrio $\bar{A}(h)$ por

$$
\bar{A}(h)=\alpha \lim _{S \rightarrow \mathbb{Z}^{2}} \mathbb{E}_{\mu_{S}}\left(X^{(0)} ; h, \beta\right)+(1-\alpha)(2 \Phi(-h)-1)
$$

onde $\mathbb{E}_{\mu_{S}}\left(X^{(0)} ; h, \beta\right)$ denota a esperança matemática da atitude do especulador $(0,0) \in S$ (valor esperado do spin na origem) segundo a medida de Gibbs $\mu_{S}$ com a Hamiltoniana indicada em (4.14) (magnetização do sistema).

Pela ergodicidade do processo $\left(X_{t}\right)_{t \geq 0}$, poderemos argumentar que, após um tempo de relaxamento $t$, vale a aproximação

$$
\bar{A}_{t}(h) \simeq \bar{A}(h)
$$

onde o lado esquerdo da aproximação corresponde à média empírica de todas as atitudes de todos os agentes do processo no tempo $t$, supondo uma sobrevalorização do ativo $h$ fixa. Os fatos que justificam essa aproximação são: 
1. No caso em que $J=0$ e $\lambda=0$, i.e., $H_{t}=h$ fixo, o processo $\left(X_{t}\right)_{t \geq 0}$ das configurações de atitudes de investimento de especuladores e o processo $\left(\bar{F}_{t}\right)_{t \geq 0}$ das médias empíricas das atitudes de investimento de fundamentalistas são processos independentes. Isto porque os termos $P_{t_{k}^{(f)}}-P_{t_{k}^{(f)}}^{(f)}$ e $P_{t_{k}^{(s)}}-P_{t_{k}^{(f)}}^{(s)}$, que aparecem nas regras de decisão (4.10) e (4.11), para $H_{t}=h$ fixo, assumem a forma $h-\theta_{t_{k}^{(f)}}$ e $h-\theta_{t_{k}^{(s)}}$, onde $\theta_{t_{k}^{(f)}}$ e $\theta_{t_{k}^{(s)}}$ são variáveis aleatórias independentes. Este fato permite que estimemos $\bar{S}_{t}$ e $\bar{F}_{t}$ separadamente e aproximemos $\bar{A}_{t}$ mediante a relação $\bar{A}_{t}=\alpha \bar{S}_{t}+(1-\alpha) \bar{F}_{t}$ e as estimativas obtidas para $\bar{S}_{t}$ e $\bar{F}_{t}$.

2. Para uma grande população de agentes e $\lambda=0$, i.e., $H_{t}=h$ fixo, vale a aproximação $\bar{F}_{t} \simeq 2 \Phi(-h)-1$, após um tempo de relaxamento do processo. Isto porque a medida invariante do processo das atitudes de fundamentalistas corresponde à medida produto de variáveis aleatórias independentes e identicamente distribuídas com esperança matemática igual a $2 \Phi(-h)-1$. Portanto, pela Lei Forte dos Grandes Números, podemos admitir a referida aproximação.

3. Para uma grande população de agentes e $\lambda=0$, i.e., $H_{t}=h$ fixo, vale a aproximação $\bar{S}_{t} \simeq \mathbb{E}_{\mu_{S}}\left(X^{(0)} ; h, \beta\right)$, após um tempo de relaxamento do processo. A justificativa para essa aproximação é a seguinte: por um lado, sob ergodicidade do processo $\left(X_{t}\right)_{t \geq 0}$, a distribuição de $X_{t}$ converge para a distribuição estacionária $\mu_{S}$, quando $t \rightarrow \infty$; por outro lado, o teorema ergódico de Birkhoff garante que a média empírica de uma seqüência ergódica de variáveis aleatórias tende ao valor esperado de suas marginais quando o tamanho da seqüência destas variáveis aleatórias tende ao infinito, i.e., quando $S \rightarrow \mathbb{Z}^{2}$.

Uma vez estabelecida a aproximação (4.16), passaremos a desenvolver nossas conjecturas sobre o comportamento do modelo para o caso em que $\lambda>0$, i.e., onde $H_{t}$ varia no tempo. Nosso procedimento neste caso fará uso de uma escolha apropriada do parâmetro $\xi$ (parâmetro da distribuição exponencial do intervalo de tempo entre duas atualizações subsequientes do preço). Sobre a escolha apropriada de $\xi$, entraremos em maiores detalhes mais adiante.

Sob alta heterogeneidade das avaliações individuais sobre o valor fundamental do ativo, 
i.e., para $\beta$ abaixo de um valor crítico, aproximaremos o processo $\left(H_{t}, \bar{A}_{t}\right)_{t \geq 0}$ pelo processo

$$
\begin{gathered}
\left.\qquad H_{t}, \bar{A}\left(H_{t}\right)\right) \\
\text { onde } \\
H_{t}=H_{t_{k}^{(m)}}+\lambda \bar{A}\left(H_{t_{k}^{(m)}}\right) \quad \forall t \in\left(t_{k}^{(m)}, t_{k+1}^{(m)}\right], \quad k=0,1,2, \ldots
\end{gathered}
$$

onde $\bar{A}\left(H_{t}\right)$ é o excesso de demanda de equilíbrio, definido em (4.15), para sobrevalorização do ativo fixada em $h=H_{t}$.

O comportamento deste processo é de fácil análise e converge $(0,0)$ para $\lambda$ e $\beta$ suficientemente pequenos. Mediante uma escolha apropriada de $\xi$, que também será justificada do ponto de vista socioeconômico, concluiremos que o processo original e o processo auxiliar não divergem muito. A partir da semelhança dos dois processos, conjecturaremos que $H_{t}$ é atraído para 0, i.e., que, essencialmente sob alta heterogeneidade das avaliações individuais sobre o valor fundamental do ativo, o preço do ativo $P_{t}\left(P_{t}=\bar{P}+H_{t}\right)$ é atraído para a avaliação média sobre o valor fundamental do ativo $\bar{P}$.

Sob baixa heterogeneidade das avaliações individuais sobre o valor fundamental do ativo, i.e., para $\beta$ acima de $\beta$ crítico, a abordagem acima apresentada será parcialmente modificada. Consideraremos o fenômeno de histerese e derivaremos um dos seguintes comportamentos para o processo $\left(H_{t}, \bar{A}_{t}\right)_{t \geq 0}$ : se $\alpha$ for relativamente grande, conjecturaremos que o processo $\left(H_{t}, \bar{A}_{t}\right)_{t \geq 0}$ apresentará um comportamento cíclico semelhante ao observado no caso análogo ao modelo de campo médio, caso esse em que tínhamos: $\alpha>\alpha_{*}$; quando a fração de especuladores $\alpha$ for positiva, mas relativamente pequena, conjecturaremos que o processo $\left(H_{t}, \bar{A}_{t}\right)_{t \geq 0}$ apresentará dois equilíbrios metaestáveis, $E_{-}=\left(-h_{\gamma}, 0\right) \mathrm{e}$ $E_{+}=\left(h_{\gamma}, 0\right)$, de forma análoga ao correspondente caso no modelo de campo médio, em que $\alpha \leq \alpha_{*}$. Sempre que $\left(H_{t}, \bar{A}_{t}\right)$ atingir o "domínio de atração" de um dos dois equilíbrios, $E_{-}$ou $E_{+}$, a correspondente sobrevalorização do ativo $H_{t}$ permanecerá flutuando próximo à sobrevalorização metaestável, $-h_{\gamma}$ ou $h_{\gamma}$, por um tempo aproximadamente exponencial. A estas sobrevalorizações metaestáveis do ativo, correspondem dois preços metaestáveis de equilíbrio de mercado, dados por $\bar{P}_{-}=\bar{P}-h_{\gamma}$ e $\bar{P}_{+}=\bar{P}+h_{\gamma}$. 


\subsubsection{Caso $J=0, K \geq 0$ e $\lambda=0$ ( $H_{t}=h$ não varia no tempo)}

Nesta seção derivaremos a medida invariante $\mu_{S}$ do processo das configurações de atitudes de investimento de especuladores $\left(X_{t}\right)_{t \geq 0}$ para $\lambda=0$, i.e., para uma sobrevalorização fixa do ativo, onde $H_{t}=h:=H_{0}, \forall t \geq 0$. Em seguida, definiremos o excesso de demanda de equilíbrio de mercado $\bar{A}(h)$ como sugerido em (4.15), i.e., a definição de $\bar{A}(h)$ será dada pela esperança matemática da atitude do especulador $(0,0)$ para um número infinito de especuladores (magnetização do sistema no limite termodinâmico).

\subsubsection{Formalização do processo estocástico a ser estudado e definições dos principais conceitos}

Vamos derivar inicialmente as taxas de mudança de atitude de investimento dos especuladores. Para tanto, observemos inicialmente o seguinte: para $J=0, K \geq 0$ e $\lambda=0$, a atitude de investimento de um especulador $s \in S$ no tempo $t+d t$ (imediatamente posterior a $t$ ), dado que o especulador $s$ atualiza sua decisão no tempo $t$, é dada por

$$
X_{t+d t}^{(s)}=\left\{\begin{array}{lll}
+1 & \text { se } & K \bar{S}_{t}^{(s)}-h>-\theta_{t}^{(s)} \\
-1 & & \text { caso contrário }
\end{array}\right.
$$

Se $K \bar{S}_{t}^{(s)}-h \neq-\theta_{t}^{(s)}$, então, como $X_{t}^{(s)}$ é igual a +1 ou -1 , temos também

$$
X_{t+d t}^{(s)}=\left\{\begin{array}{lll}
+X_{t}^{(s)} & \text { se } & X_{t}^{(s)}\left[\left(K \bar{S}_{t}^{(s)}-h\right)\right]>X_{t}^{(s)}\left[-\theta_{t}^{(s)}\right] \\
-X_{t}^{(s)} & \text { caso contrário }
\end{array}\right.
$$

Levando em conta que $\theta_{t}^{(s)}$ é distribuída simetricamente em torno do 0 mediante uma função de distribuição de probabilidade acumulada $\Phi$ e que $X_{t}^{(s)} \in\{-1,1\}$, temos que $X_{t}^{(s)}\left[-\theta_{t}^{(s)}\right]$ tem a mesma distribuição de probabilidade que $\theta_{t}^{(s)}$. Como $\Phi$ é contínua, o evento $\left\{K \bar{S}_{t}^{(s)}-h=-\theta_{t}^{(s)}\right\}$ ocorre com probabilidade zero. Portanto, dado que um especulador $s \in S$ atualiza sua atitude no tempo $t$, a probabilidade de mudança de atitude de investimento de $s$ no tempo $t$ é dada por

$$
1-\Phi\left(X_{t}^{(s)}\left[\left(K \bar{S}_{t}^{(s)}-h\right)\right]\right)
$$

Em outras palavras, o processo $\left(X_{t}\right)_{t \geq 0}\left(X_{t} \in\{-1,1\}^{S}\right)$ é um processo em tempo contínuo com taxas de mudança de valor de $X^{(s)} \in\{-1,1\}, s \in S$, dadas por

$$
C(s, X):=1-\Phi\left(X^{(s)}\left[\left(K \bar{S}^{(s)}-h\right)\right]\right)=\frac{1}{1+\exp \left(2 \beta X^{(s)}\left[K \bar{S}^{(s)}-h\right]\right)}
$$


para $\forall s \in S$ e $\forall X \in\{-1,1\}^{S}$, onde $X^{(s)} \in\{-1,1\}$ é o valor atribuído ao sítio $s \in S \subset \mathbb{Z}^{2}$, e $\bar{S}^{(s)}$ é a média aritmética dos vizinhos mais próximos de $s \in S$, dada a configuração $X$. A segunda igualdade de (4.18) segue imediatamente da forma específica de $\Phi$ que postulamos em (4.6).

As taxas $C(s, X), s \in S, X \in\{-1,+1\}^{S}$, apresentadas em (4.18), revelam que o processo estocástico $\left(X_{t}\right)_{t \geq 0}$ é um processo estocástico de Ising com respeito a um potencial unicamente determinado pela expressão $\beta X^{(s)}\left[K \bar{S}^{(s)}-h\right]$, i.e., onde $\beta K / 4$ é o coeficiente associado a conjuntos de sítios formados por pares de sítios conectados por elos em $S$ e onde $-h$ é o coeficiente associado a conjuntos de sítios formados por um único sítio em $S$. Sobre processos estocásticos de Ising temos muitas informações, veja Capítulo 4 de T. M. Liggett [17]. Em particular para $S$ finito, o processo é ergódico e a medida invariante do processo é dada pela medida de Gibbs $\mu_{S}$ como segue abaixo:

$$
\mu_{S}(X)=\frac{\exp \left\{\beta \sum_{s \in S} X^{(s)}\left[K \bar{S}^{(s)}-h\right]\right\}}{Z}
$$

onde $Z$ é a constante normalizadora, i.e.,

$$
Z:=\sum_{X \in\{-1,1\}^{S}} \exp \left\{\beta \sum_{s \in S} X^{(s)}\left[K \bar{S}^{(s)}-h\right]\right\}
$$

Observamos que a medida $\mu_{S}$ corresponde à medida de Gibbs do modelo de Ising ferromagnético $(K>0)$. A constante de interação entre spins vizinhos é $K / 4$, i.e., diretamente proporcional à influência social direta; o campo externo é igual à subvalorização do ativo $-h$; e a temperatura é diretamente proporcional à heterogeneidade das avaliações individuais sobre o valor fundamental do ativo, i.e., $1 / \beta=\left(2 \Phi^{\prime}(0)\right)^{-1}$.

Excesso de demanda de equilíbrio de especuladores. Seja $\mathbb{E}_{\mu_{S}}\left(X^{(0)} ; h, \beta\right)$ a esperança matemática de $X^{(0)}$ segundo a medida $\mu_{S}$ dada em (4.19). Como se sabe, existe um valor crítico $\beta_{c}$, tal que o limite

$$
\bar{S}(h):=\lim _{S \rightarrow \mathbb{Z}^{2}} \mathbb{E}_{\mu_{S}}\left(X^{(0)} ; h, \beta\right)
$$

está bem definido para $h \neq 0$ ou $\beta<\beta_{c}$. Chamaremos o limite acima de excesso de demanda de equilíbrio de especuladores. 
Excesso de demanda de equilíbrio. Definiremos, para $h \neq 0$, ou $\beta<\beta_{c}$, ou $\alpha=0$, o excesso de demanda de equilíbrio $\bar{A}(h)$ por

$$
\bar{A}(h):=\alpha \bar{S}(h)+(1-\alpha)(2 \Phi(-h)-1)
$$

A razão para definirmos o excesso de demanda de equilíbrio $\bar{A}(h)$ segundo (4.22) vem da convergência de $\bar{A}_{t}$ para $\bar{A}(h)$. Mais especificamente, para $h \neq 0$, ou $\beta<\beta_{c}$, ou $\alpha=0$, vale

$$
\lim _{N_{S} / N=\alpha ; N \rightarrow \infty}\left[\lim _{t \rightarrow \infty} \mathbb{P}_{S, F}\left(\left|\bar{A}_{t}-\bar{A}(h)\right|>\varepsilon\right)\right]=0
$$

onde $\mathbb{P}_{S, F}\left(\left|\bar{A}_{t}-\bar{A}(h)\right|>\varepsilon\right)$ denota a probabilidade de $\left|\bar{A}_{t}-\bar{A}(h)\right|>\varepsilon, F=$ $\left\{1,2, \ldots N_{F}\right\}$ denota o conjunto de fundamentalistas e $S, S \subset \mathbb{Z}^{2}$, denota o conjunto de especuladores, apresentado em (4.9) ( $S$ e $F$ fixos para todos os tempos $\tau=0,1, \ldots t$ ).

A convergência (4.23) pode ser facilmente deduzida da igualdade dada por $\bar{A}_{t}=$ $\frac{\sum_{i \in A} X_{t}^{(i)}}{N}=\frac{N_{S}}{N} \frac{\sum_{s \in S} X_{t}^{(s)}}{N_{S}}+\frac{N_{F}}{N} \frac{\sum_{f \in F} X_{t}^{(f)}}{N_{F}}(t=0,1,2, \ldots)$, e da ergodicidade do processo estocástico das configurações das atitudes de especuladores $\left(X_{t}^{(s)}, s \in S\right)_{t \geq 0}$.

\subsubsection{Descontinuidade do excesso de demanda relativo}

Nesta seção concluiremos o comportamento de $\bar{A}(h)$ enquanto função de $h$ para diferentes valores da heterogeneidade das avaliações individuais sobre o valor fundamental do ativo $\left(\Phi^{\prime}(0)\right)^{-1}$.

Sabemos que a função $h \mapsto \bar{S}(h)$ é ímpar, decrescente e contínua em $\mathbb{R}-\{0\}$. Além disso, para $\beta<\beta_{c}, \bar{S}(h)$ é também contínua em 0 ; e, para $\beta>\beta_{c}$, vale

$$
\lim _{h \rightarrow-0} \bar{S}(h)>0>\lim _{h \rightarrow+0} \bar{S}(h)
$$

Como a função $h \mapsto 2 \Phi(-h)-1$ é ímpar, decrescente e contínua em todo $\mathbb{R}$, o excesso de demanda de equilíbrio $\bar{A}(h)(\bar{A}(h)=\alpha \bar{S}(h)+(1-\alpha)(2 \Phi(-h)-1))$ satisfaz:

1. Se $\left(\Phi^{\prime}(0)\right)^{-1}>\phi(K)$ ou $\alpha=0$

então $\bar{A}(h)$ é decrescente, ímpar e contínua em $h \in \mathbb{R}$; 


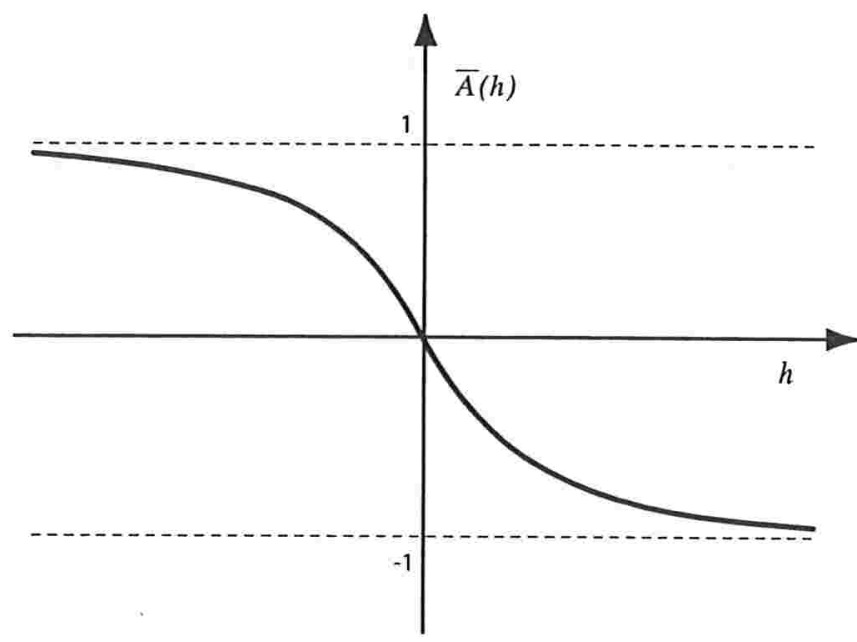

Figura 4.1: Caso $\left(\Phi^{\prime}(0)\right)^{-1}>\phi(K)$ ou $\alpha=0$. Gráfico de $\bar{A}(h)$.

2. Se $\left(\Phi^{\prime}(0)\right)^{-1}<\phi(K)$ e $\alpha>0$

então $\bar{A}(h)$ é decrescente, ímpar e contínua em $h \in \mathbb{R}-\{0\}$, onde vale

$$
\lim _{h \rightarrow-0} \bar{A}(h)=: \bar{A}_{+}(0)>0>\bar{A}_{-}(0):=\lim _{h \rightarrow+0} \bar{A}(h)
$$

onde $\phi(K):=2 / \beta_{c}(K)$ é crescente em $K \operatorname{com} \phi(0)=0$.

As desigualdades $\left(\Phi^{\prime}(0)\right)^{-1}>\phi(K)$ e $\left(\Phi^{\prime}(0)\right)^{-1}<\phi(K)$, mencionadas nos casos 1) e 2) acima, são equivalentes às desigualdades $\beta<\beta_{c}$ e $\beta>\beta_{c}$, respectivamente. Isto porque $\left(\Phi^{\prime}(0)\right)^{-1}=2 / \beta$, onde o valor crítico $\beta_{c}=\beta_{c}(K)$ é uma função decrescente de $K$, com $\lim _{K \rightarrow+0} \beta_{c}(K)=\infty$. Optamos pelas desigualdades $\left(\Phi^{\prime}(0)\right)^{-1}>\phi(K)$ e $\left(\Phi^{\prime}(0)\right)^{-1}<\phi(K)$ a fim de viabilizar a analogia com os resultados obtidos com o modelo apresentado no Capítulo 2 (modelo de campo médio), bem como permitir o mesmo tipo de interpretação socioeconômica apresentada no contexto daquele modelo. Dependendo da relação entre $\left(\Phi^{\prime}(0)\right)^{-1}$ (heterogeneidade das avaliações individuais sobre valor fundamental do ativo) e $K$ (influência social direta), teremos um ou outro comportamento do excesso de demanda de equilíbrio $\bar{A}(h)$, enquanto função de $h$ (veja as figuras 4.1 e 4.2).

\subsubsection{Histerese}

As interpretações socioeconômicas que podemos derivar das propriedades do excesso de demanda de equilíbrio $\bar{A}(h)$, a princípio, diferem daquelas apresentadas no contexto do 


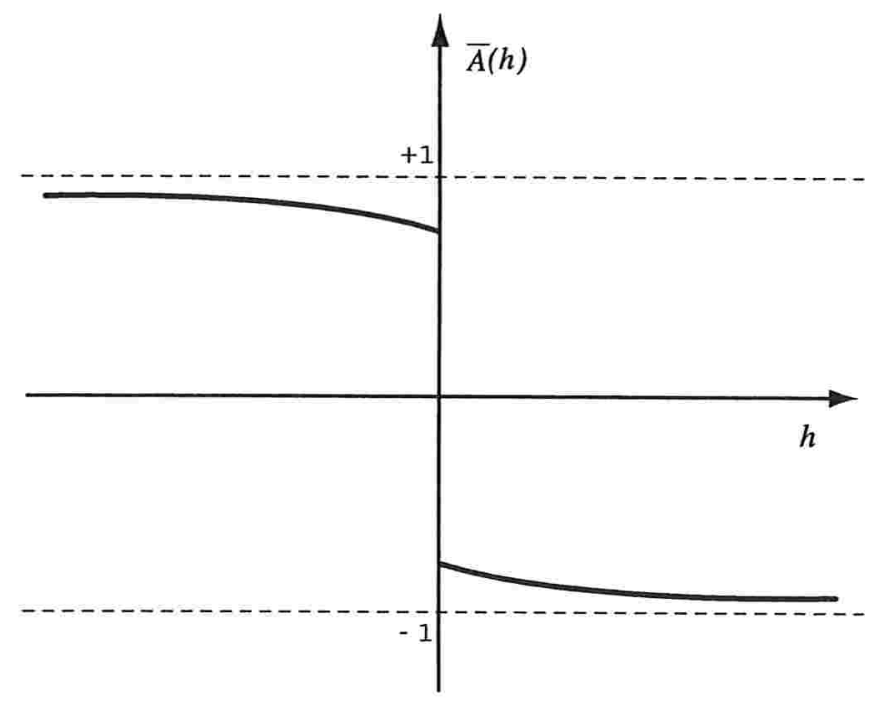

Figura 4.2: Caso $\left(\Phi^{\prime}(0)\right)^{-1}<\phi(K)$ e $\alpha>0$. Gráfico de $\bar{A}(h)$.

modelo do Capítulo 2. A diferença está no ponto de descontinuidade do excesso de demanda $\bar{A}(h)$, no caso $\left(\Phi^{\prime}(0)\right)^{-1}<\phi(K)$ e $\alpha>0$, i.e., sob baixa heterogeneidade das avaliações individuais sobre o valor fundamental do ativo e sob uma fração positiva de especuladores no mercado. Neste caso, i.e., sob $\left(\Phi^{\prime}(0)\right)^{-1}<\phi(K)$ e $\alpha>0$, a função $h \mapsto \bar{A}(h)$ é descontínua em zero.

Ocorre que evidências empíricas, baseadas em simulações de processos de Ising estocásticos, sugerem a existência de estados metaestáveis do sistema. Isto é, se simularmos o processo estocástico das configurações de atitudes de especuladores $\left(X_{t}\right)_{t \geq 0}$ para uma sobrevalorização $h<0$ fixa do ativo (campo externo $=-h>0$ ) e, após algum tempo (após a média empírica $\bar{S}_{t}=\sum_{s \in S} X_{t}^{(s)} / N_{S}$ ter atingido um valor bem próximo à magnetização do sistema em $\bar{S}(h)$ ), interviermos no processo variando a sobrevalorização do ativo de $h$ para um valor positivo $h+\Delta h$, veremos então que $\bar{S}_{t}$ assume rapidamente um valor $\bar{S}_{+}(h+\Delta h)$ intermediário positivo, onde $\bar{S}(h)>\bar{S}_{+}(h+\Delta h)>0>\bar{S}(h+\Delta h)$. Somente após um tempo aproximadamente exponencial, o processo entra em equilíbrio, onde então a média empírica $\bar{S}_{t}$ passa a flutuar em torno da nova magnetização do sistema $\bar{S}(h+\Delta h)$ (excesso de demanda de equilíbrio de especuladores). Este fenômeno é conhecido como fenômeno de histerese. Do ponto de vista de uma descrição física do fenômeno, a distribuição aproximadamente exponencial do tempo decorre do fato da média empírica 
intermediária $\bar{S}_{+}(h+\Delta h)$ corresponder a estados metaestáveis (mínimos locais de energia), para onde o sistema é essencialmente atraído várias vezes antes de sair. Como o processo é Markoviano, a cada retorno o processo perde essencialmente a memória e a contagem do tempo recomeça.

Uma caracterização matemática do fenômeno de metaestabilidade para sistemas de spins ferromagnéticos pode ser encontrada nas contribuições de E. J. Neves e R. H. Schonmann [26] e [27]. A análise desenvolvida para o fenômeno de metaestabilidade estuda a formação de gotas droplets de spins -1 (em nosso caso correspondendo a agrupamentos de atitudes de investimento de venda) em um "mar" de spins +1 , que freqüentemente retraem antes de atingir um tamanho crítico. A partir do momento em que uma gota crítica se forma, cujo tamanho é função decrescente de $h>0$, esta gota de spins negativos tende a crescer irreversivelmente, dando lugar à formação de configurações caracterizadas por um mar de spins -1 com pequenas gotas de spins +1 . Ou seja, após um tempo exponencial necessário para que uma gota crítica de spins -1 ocorra, a média empírica corrente $\bar{S}_{t}$ é atraída rapidamente para a magnetização de equilíbrio do processo em $\bar{S}(h+\Delta h)$ (magnetização negativa).

A interpretação socioeconômica que podemos derivar do fenômeno de histerese sugere que, quando a sobrevalorização do ativo se torna positiva, o excesso de demanda persiste positivo durante um tempo aleatório aproximadamente exponencial, cujo valor esperado será tanto menor quanto maior for a sobrevalorização do ativo $h$. Observamos que essa idéia é compatível com a noção de risco iminente de crash de preço do ativo: quanto mais sobrevalorizado estiver o ativo tanto menor será o tempo de espera para a ocorrência de um crash de preço. Observamos ainda que, quando $h=0$ e $\beta>\beta_{c}$, o processo $\left(X_{t}\right)_{t \geq 0}$ não é ergódico e admite duas medidas invariantes extremais. Assim, quando a avaliação média corresponder ao preço do ativo $(h=0)$, haverá sempre um excesso de oferta $\left(\bar{A}_{-}(h)<0\right)$ ou de procura $\left(\bar{A}_{+}(h)>0\right)$ diferente de zero. Este fato mostra que, sob baixa heterogeneidade das avaliações individuais sobre o valor fundamental do ativo e presença de especuladores no mercado, a avaliação média $\bar{P}$ não pode ser um preço de equilíbrio de mercado estável do ativo.

Mostraremos, mais adiante, que, se a fração de especuladores $\alpha$ for suficientemente pequena, porém positiva, existirão dois preços de equilíbrio de mercado metaestáveis 
do ativo diferentes da avaliação média $\bar{P}$. O termo "metaestável" decorre do fato de os referidos preços de equilíbrio estarem associados a estados metaestáveis do processo estocástico das configurações das atitudes de investimento de especuladores.

\subsubsection{Sobrevalorização crítica do ativo $h_{0}$}

Com base na exposição acima, vamos agora apresentar a sobrevalorização crítica do ativo $h_{0}>0$ no caso $\left(\Phi^{\prime}(0)\right)^{-1}<\phi(K)$ e $\alpha>0$. A definição da sobrevalorização crítica $h_{0}$ corresponde à definção de $h_{*}$, i.e., definição da sobrevalorização crítica do ativo no contexto do modelo do Capítulo 2.

Levando em conta que o fenômeno de histerese é um fenômeno temporal, não é possível definir $h_{0}$ como um limiar positivo sem considerarmos a variável "tempo". Isto porque, para qualquer $h$ positivo, o excesso de demanda de equilíbrio é único; i.e., mais cedo ou mais tarde, $\bar{A}_{t}$ será atraído para $\bar{A}(h)$, qualquer que seja $h>0$.

A fim de apresentar $h_{0}$, vamos considerar o processo das configurações de atitudes de investimento de especuladores $\left(X_{t}\right)_{t \geq 0}$ para uma sobrevalorização fixa do ativo em $h$, i.e., $\lambda=0$ e $H_{0}=h$. Para $h>0$ e $\bar{S}_{0}=\bar{S}_{+}(h)$, vamos definir $T(h)$ como sendo o tempo de flutuação de $\bar{S}_{t}$ em torno de $\bar{S}_{+}(h)$. Este tempo corresponde ao tempo de espera necessário para que uma gota crítica de spins negativos se forme e a média empírica $\bar{S}_{t}$ abandone o domínio de atração da magnetização metaestável $\bar{S}_{+}(h)$ e seja atraída para a magnetização do sistema $\bar{S}(h)$. Vamos aproximar a distribuição de probabilidades do tempo $T(h)$ por uma distribuição exponencial, cujo parâmetro é função crescente de $h$. Para a esperança matemática de $T(h)$, denotada por $\mathbb{E}(T(h))$, admitiremos a existência de $\operatorname{um} h_{0}>0$, tal que:

1. $h \mapsto \mathbb{E}(T(h))$ é estritamente decrescente em $\left[0, h_{0}\right]$; e

2. $\mathbb{E}(T(h))=0, \forall h \in\left[h_{0}, \infty\right)$.

Definimos a sobrevalorização crítica $h_{0}$ como sendo aquele valor positivo que satisfaz as afirmações 1) e 2) acima mencionadas.

O objetivo desta definição, em última análise, é introduzir a conjectura de um preço metaestável do ativo, baseada na noção de um conjunto de magnetizações metaestáveis, dada pela união de todos os pontos $\left(h, \bar{S}_{+}(h)\right)$, onde $h \in\left[0, h_{0}\right]$. Vamos admitir que 
$\bar{S}_{+}(h)$ seja contínua e decrescente para $h \in\left[0, h_{0}\right]$ e vamos definir o excesso de demanda metaestável superior por

$$
\bar{A}_{+}(h):=\alpha \bar{S}_{+}(h)+(1-\alpha)(2 \Phi(-h)-1), \quad \forall h \in\left[0, h_{0}\right]
$$

De forma análoga, definimos o excesso de demanda metaestável inferior $\bar{A}_{+}(h)$ para $h \in$ $\left[-h_{0}, 0\right]$.

Negatividade do excesso de demanda metaestável superior. Analogamente ao modelo de campo médio, teremos $\bar{A}_{+}\left(h_{0}\right)<0$ desde que a heterogeneidade das avaliações individuais sobre o valor fundamental do ativo seja suficientemente baixa, i.e., $\left(\Phi^{\prime}(0)\right)^{-1}<$ $\phi(K)$ e $\alpha$ suficientemente pequeno, porém positivo, ou seja,

$$
0<\alpha<\alpha_{*}:=\frac{\left[2 \Phi\left(h_{0}\right)-1\right]}{\bar{S}_{+}\left(h_{0}\right)+\left[2 \Phi\left(h_{0}\right)-1\right]} \quad<1
$$

Ou seja, se $\left(\Phi^{\prime}(0)\right)^{-1}<\phi(K)$ e $0<\alpha<\alpha_{*}$, existe um $h_{\gamma}$, tal que:

1. $0<h_{\gamma}<h_{0}$;

2. $\bar{A}_{+}\left(h_{\gamma}\right)=0>\bar{A}_{+}\left(h_{0}\right)$;

3. $\gamma:=\mathbb{E}\left(T\left(h_{\gamma}\right)\right)>\mathbb{E}\left(T\left(h_{0}\right)\right)=0$.

Este fato joga um papel crucial na existência de dois preços de equilíbrio metaestáveis de mercado quando considerarmos $\lambda>0$.

Observamos que o item 3) segue da monotonicidade de $h \mapsto \mathbb{E}(T(h))$ e devido a $h_{0}=\operatorname{Inf}\left\{h>0 \mid \mathbb{E}(T(h)=0\}\right.$. Observamos ainda que as notações $h_{\gamma}$ e $h_{0}$ foram escolhidas de maneira a sugerir as esperanças matemáticas $\gamma$ e 0 dos respectivos valores esperados de $T\left(h_{\gamma}\right)$ e $T\left(h_{0}\right)$, respectivamente (veja as figuras 4.3 e 4.4 ).

\subsubsection{Caso $J=0, K \geq 0$ e $\lambda>0$ ( $H_{t}$ varia no tempo)}

Apresentaremos agora uma conjectura sobre o comportamento do processo para a região de parâmetros acima mencionada. Esta conjectura e os termos que empregamos em sua formulação serão explicados em mais detalhes na seção seguinte. 


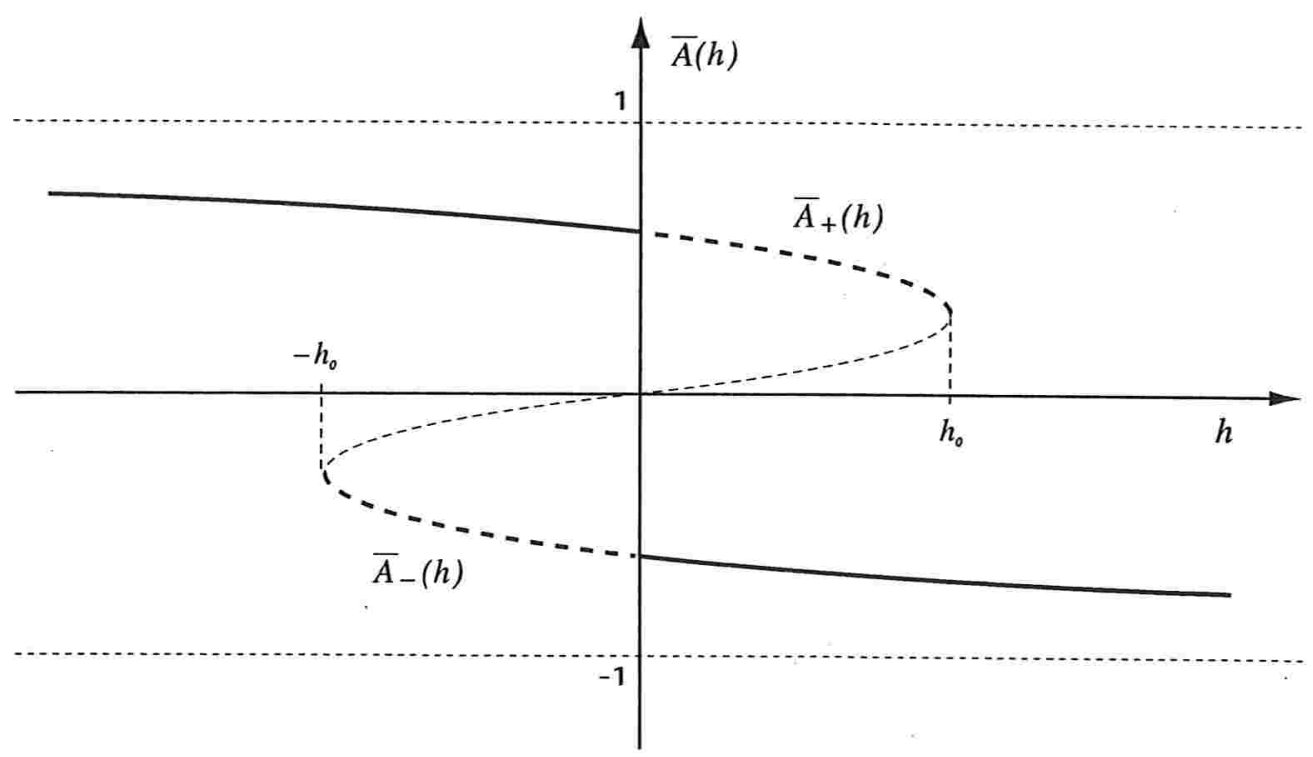

Figura 4.3: Caso $\left(\Phi^{\prime}(0)\right)^{-1}<\phi(K)$ e $\alpha>\alpha_{*}$. Gráficos de $\bar{A}_{-}(h)$ e $\bar{A}_{+}(h)$ enquanto extensões do gráfico de $\bar{A}(h)$.

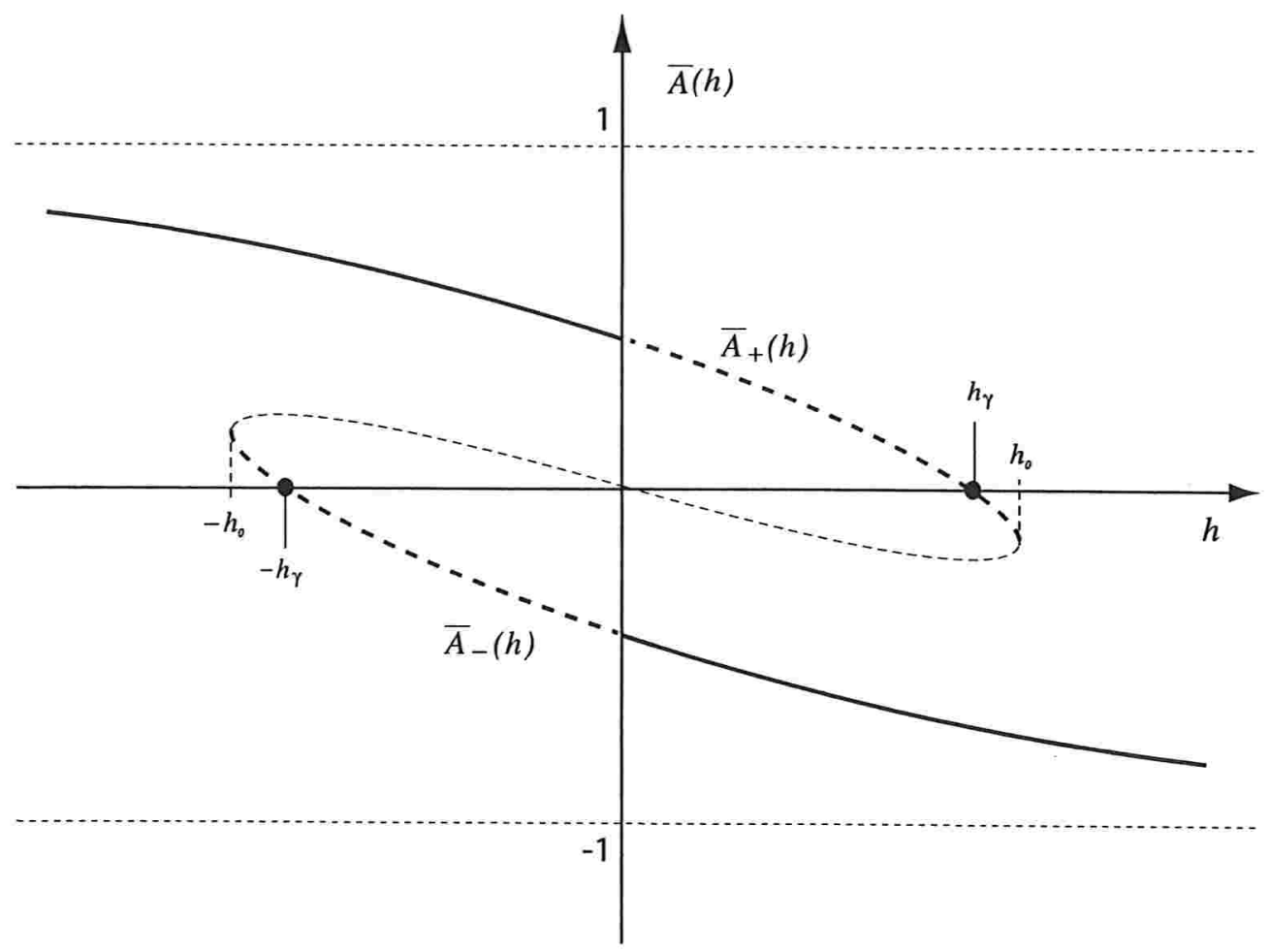

Figura 4.4: Caso $\left(\Phi^{\prime}(0)\right)^{-1}<\phi(K)$ e $0<\alpha<\alpha_{*}$. Gráficos de $\bar{A}_{-}(h)$ e $\bar{A}_{+}(h)$ enquanto extensões do gráfico de $\bar{A}(h)$. 
Conjectura 2. Seja $\left(H_{t}, \bar{A}_{t}\right)_{t \geq 0}$ o processo estocástico determinado pelo processo $\left(W_{t}\right)_{t \geq 0}$ em (4.12). Então, conjecturamos que, para $\lambda$ suficientemente pequeno e $\xi$ (parâmetro da distribuição do tempo de atualização do preço) apropriado, vale:

1. Se $\left(\Phi^{\prime}(0)\right)^{-1}>\phi(K)$ ou $\alpha=0$

então $\left(H_{t}, \bar{A}_{t}\right)$ é atraído rapidamente para $(0,0)$, qualquer que seja a condição inicial $\left(H_{0}, \bar{A}_{0}\right)$.

2. $S e\left(\Phi^{\prime}(0)\right)^{-1}<\phi(K)$

então existe um $\alpha_{*}>0$, tal que, se alternativamente valer:

(a) $0<\alpha<\alpha_{*}$

então $\left(H_{t}, \bar{A}_{t}\right)$ possui dois equilibrios metaestáveis, denotados por $E_{+}$e $E_{-}$, onde $E_{-}=-E_{+}$com $E_{+}:=\left(h_{\gamma}, 0\right)$, e onde $0<h_{\gamma}<h_{0}$. Para estes equilibrios metaestáveis, o tempo de permanência é aproximadamente exponencial, cujo valor esperado $\gamma$ satisfaz

$$
\gamma:=\mathbb{E}\left(T\left(h_{\gamma}\right)\right)>\mathbb{E}\left(T\left(h_{0}\right)\right)=0 \quad\left(\text { uma vez que } h_{\gamma}<h_{0}\right)
$$

(b) $\alpha>\alpha_{*}$

então $\left(H_{t}, \bar{A}_{t}\right)_{t \geq 0}$ não possui equilíbrios metaestáveis. Neste caso, $\left(H_{t}, \bar{A}_{t}\right)_{t \geq 0}$ apresenta um comportamento cíclico (correspondendo à formaçâo de bolhas especulativas e crashes de preço do ativo).

\subsubsection{Embasamento da conjectura e a escolha apropriada dos tempos de atualização do preço}

Para desenvolver os argumentos que justificam a Conjectura 2, vamos inicialmente extender o domínio de definição da função $\bar{A}_{+}(\cdot)$ de $\left[0, h_{0}\right]$ para $\left(-\infty, h_{0}\right]$, onde definimos $\bar{A}_{+}(h):=\bar{A}(h)$ para $h \in(-\infty, 0)$. Da mesma forma, definiremos $\bar{A}_{-}(h):=\bar{A}(h)$ para $h \in(0, \infty)$. As figuras $4.5,4.6$ e 4.7 a seguir ilustram o comportamento aproximado de $\left(H_{t}, \bar{A}_{t}\right)_{t \geq 0}$.

Os argumentos que justificam a dinâmica de $\left(H_{t}, \bar{A}_{t}\right)_{t \geq 0}$, conforme ilustrado nas figuras citadas, são análogos àqueles empregados nos casos correspondentes ao modelo apresentado no Capítulo 2. 


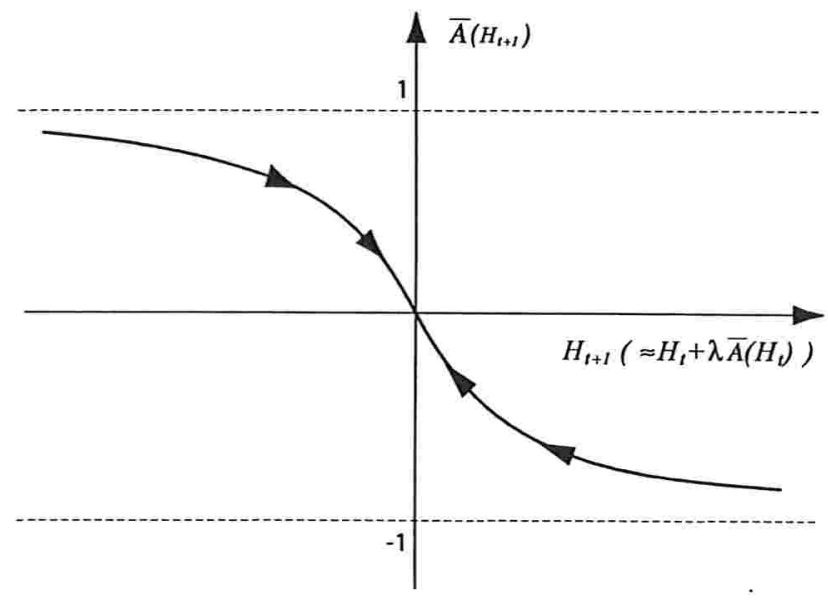

Figura 4.5: Caso $\left(\Phi^{\prime}(0)\right)^{-1}>\phi(K)$ ou $\alpha=0 .\left(H_{t}, \bar{A}_{t}\right)_{t \geq 0}$ é atraído para $(0,0)$.

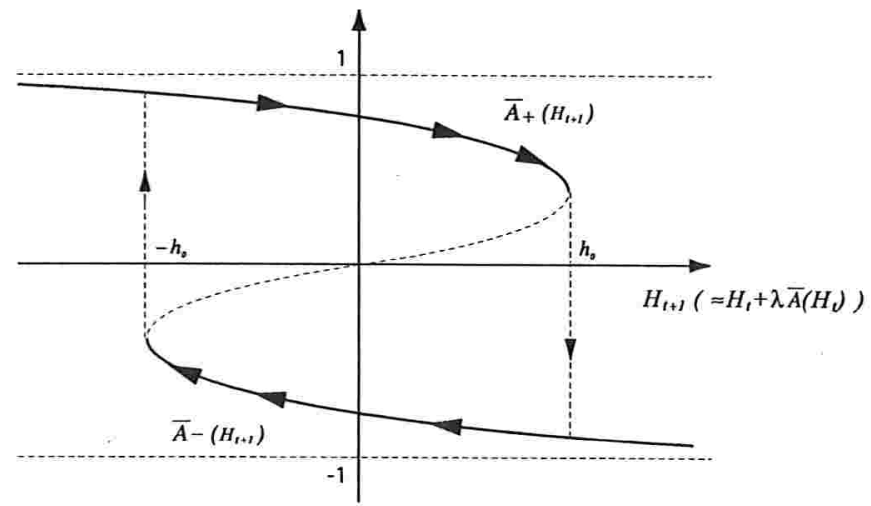

Figura 4.6: Caso $\left(\Phi^{\prime}(0)\right)^{-1}<\phi(K)$ e $\alpha>\alpha_{*}$. $\left(H_{t}, \bar{A}_{t}\right)_{t \geq 0}$ apresenta um comportamento cíclico.

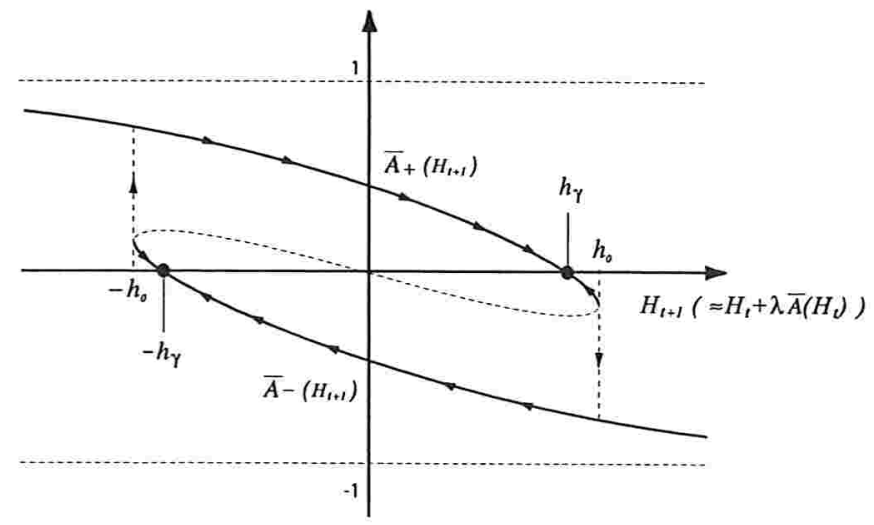

Figura 4.7: Caso $\left(\Phi^{\prime}(0)\right)^{-1}<\phi(K)$ e $0<\alpha<\alpha_{*}$. $\left(H_{t}, \bar{A}_{t}\right)_{t \geq 0}$ é atraído para $\left(-h_{\gamma}, 0\right)$ ou para $\left(h_{\gamma}, 0\right)$. 
Vamos esclarecer agora a questão da escolha apropriada do parâmetro $\xi$. Lembramos que $\xi$ é o parâmetro da distribuição exponencial das variáveis aleatórias $t_{k+1}^{(m)}-t_{k}^{(m)}, k=$ $0,1,2, \ldots$ (tempos decorridos entre dois ajustes subseqüentes do preço).

Vamos escolher $1 / \xi$ (tempo médio decorrido entre duas atualizações de preço) tal que o mesmo exceda, porém não excessivamente, o tempo médio de espera necessário para que a medida limite do processo $X_{t}$ se estabeleça, sob $\left(\Phi^{\prime}(0)\right)^{-1}>\phi(K)$ e $\lambda=0, H_{0}=h$. Lembramos que, sob $\left(\Phi^{\prime}(0)\right)^{-1}>\phi(K)$ e $\lambda=0, H_{0}=h$, o processo $X_{t}$ das configurações de atitudes de investimento de especuladores é ergódigo e possui, portanto, uma única medida invariante para quaisquer valores de $h \in \mathbb{R}$.

A seguir, sob a escolha de $\xi$ como indicada acima, justificaremos a aproximação do processo $\left(H_{t}, \bar{A}_{t}\right)_{t \geq 0}$, conforme indicado com as figuras $4.5,4.6$ e 4.7 .

Vamos inicialmente justificar a aproximação do processo $\left(H_{t}, \bar{A}_{t}\right)_{t \geq 0}$ no caso $\left(\Phi^{\prime}(0)\right)^{-1}>$ $\phi(K)$ ou $\alpha=0$, conforme ilustrado na Figura 4.5. Como o tempo médio entre duas atualizações de preço $1 / \xi$ é da ordem do tempo de espera necessário para que a medida invariante $\mu_{S}$ se estabeleça, podemos, neste caso, aproximar o processo $\left(H_{t}, \bar{A}_{t}\right)_{t \geq 0}$ ao processo $\left(H_{t}, \bar{A}\left(H_{t}\right)\right)_{t \geq 0}$, restrito aos termos de atualização de preço $t_{k}^{(m)}, k=0,1,2, \ldots$, i.e.,

$$
\left(H_{t_{k}^{(m)}}, \bar{A}_{t_{k}^{(m)}}\right) \simeq\left(H_{t_{k}^{(m)}}, \bar{A}\left(H_{t_{k}^{(m)}}\right)\right), \quad k=0,1,2, \ldots
$$

onde $\bar{A}\left(H_{t_{k}^{(m)}}\right)$ corresponde ao excesso de demanda de equilíbrio para a sobrevalorização fixa do ativo $H_{t_{k}^{(m)}}$, i.e., onde

$$
\bar{A}\left(H_{t_{k}^{(m)}}\right):=\alpha \lim _{S \rightarrow \mathbb{Z}^{2}} \mathbb{E}_{\mu_{S}}\left(X^{(0)} ; H_{t_{k}^{(m)}}, \beta\right)+(1-\alpha)(2 \Phi(-h)-1)
$$

para $k=0,1,2, \ldots$

Podemos admitir a aproximação (4.27), pois, sob a escolha de $\xi$ mencionada, a sobrevalorização do ativo é atualizada somente após o excesso de demanda de equilíbrio $\bar{A}\left(H_{t}\right)$ ter se estabelecido, i.e., somente após $\bar{A}_{t} \simeq \bar{A}\left(H_{t}\right)$. Agora, como $H_{t_{k+1}^{(m)}}=H_{t_{k}^{(m)}}+$ $\lambda \bar{A}_{t_{k}^{(m)}} \simeq H_{t_{k}^{(m)}}+\lambda \bar{A}\left(H_{t_{k}^{(m)}}\right)$ e, no caso $\left(\Phi^{\prime}(0)\right)^{-1}>\phi(K)$, a função $h \mapsto \bar{A}(h)$ é ímpar, decrescente e contínua, é fácil ver que o processo $\left(H_{t_{k}^{(m)}}, \bar{A}_{t_{k}^{(m)}}\right)_{k=0,1,2, \ldots}$ é atraído para $(0,0)$, quando $\lambda$ é suficientemente pequeno. Note que, como $H_{t}$ não varia durante os intervalos de tempo $\left[t_{k+1}^{(m)}, t_{k}^{(m)}\right], k=0,1,2, \ldots$, a restrição da aproximação (4.27) aos tempos $t_{k}^{(m)}$, 
$k=0,1,2, \ldots$ não implica em perda de informação sobre o processo $\left(P_{t}\right)_{t \geq 0}\left(\bar{P}_{t}=\bar{P}+H_{t}\right.$, $t \geq 0$ ).

Vamos agora justificar a aproximação do processo $\left(H_{t}, \bar{A}_{t}\right)_{t \geq 0}$ no caso $\left(\Phi^{\prime}(0)\right)^{-1}<$ $\phi(K)$, conforme ilustrado nas figuras 4.6 e 4.7. Nossa suposição aqui é que o tempo médio $1 / \xi$ é da mesma ordem de grandeza do tempo de espera necessário para que $\bar{A}_{t}$ seja atraído para $\bar{A}_{+}(h)\left(\bar{A}_{-}(h)\right)$, desde que $\bar{A}_{0}$ se encontre no domínio de atração de $\bar{A}_{+}(h)$ $\left(\bar{A}_{-}(h)\right)$, e sob a suposição $\lambda=0$ e $H_{0}=h, \forall h \in \mathbb{R}$. De forma análoga ao caso anterior, podemos justificar a aproximação $\left(H_{t_{k}^{(m)}}, \bar{A}_{t_{k}^{(m)}}\right) \simeq\left(H_{t_{k}^{(m)}}, \bar{A}_{+}\left(H_{t_{k}^{(m)}}\right)\right), k=0,1,2, \ldots$, para $\bar{A}_{0}$ pertencente ao domínio de atração de $\bar{A}_{+}\left(H_{0}\right)$ e enquanto $H_{t_{k}^{(m)}}<h_{0}$. Da mesma forma, podemos argumentar a aproximação $\left(H_{t_{k}^{(m)}}, \bar{A}_{t_{k}^{(m)}}\right) \simeq\left(H_{t_{k}^{(m)}}, \bar{A}_{-}\left(H_{t_{k}^{(m)}}\right)\right), k=0,1,2, \ldots$, para $\bar{A}_{0}$ pertencente ao domínio de atração de $\bar{A}_{-}\left(H_{0}\right)$ e enquanto $H_{t_{k}^{(m)}}>-h_{0}$.

No subcaso $\left(\Phi^{\prime}(0)\right)^{-1}<\phi(K)$ e $\alpha>\alpha_{*}$ (Figura 4.6) podemos deduzir, pelas aproximações acima mencionadas, que a sobrevaorização do ativo $H_{t}$ se movimenta para a direita ou esquerda levando o sistema sempre de um estado estável para um estado metaestável. Quando o sistema se encontra em um estado metaestável em um dado instante $t$, por exemplo $\left(H_{t}, \bar{A}_{t}\right)=\left(H_{t}, \bar{A}_{+}\left(H_{t}\right)\right), 0<H_{t}<h_{0}$, a sobrevalorização do ativo $H_{t}$ tende a crescer até que o estado metaestável seja abandonado ou, mais tardar, até que $H_{t}$ exceda $h_{0}$. A partir deste momento, $\bar{A}_{t}$ é atraído para o equilíbrio estável $\bar{A}_{-}\left(H_{t}\right)$. A partir da condição $\left(H_{t}, \bar{A}_{t}\right)=\left(H_{t}, \bar{A}_{-}\left(H_{t}\right)\right)$ esse mecanismo se repete com sinal oposto.

Já no subcaso $\left(\Phi^{\prime}(0)\right)^{-1}<\phi(K)$ e $0<\alpha<\alpha_{*}$ (Figura 4.7), partindo de $\left(H_{0}, \bar{A}_{0}\right)=$ $\left(H_{0}, \bar{A}_{+}\left(H_{0}\right)\right), H_{0}<h_{0}$, a aproximação $\left(H_{t_{k}^{(m)}}, \bar{A}_{t_{k}^{(m)}}\right) \simeq\left(H_{t_{k}^{(m)}}, \bar{A}_{+}\left(H_{t_{k}^{(m)}}\right)\right), k=0,1,2, \ldots$, indica que o processo original $\left(H_{t}, \bar{A}_{t}\right)_{t \geq 0}$ é atraído localmente para $\left(h_{\gamma}, 0\right)$. Os mecanismos que sugerem essa atração são os mesmos já comentados no caso em que $\left(\Phi^{\prime}(0)\right)^{-1}>\phi(K)$ ou $\alpha=0$. Uma vez que $\left(H_{t}, \bar{A}_{t}\right)_{t \geq 0}$ alcança o equilíbrio metaestável $\left(h_{\gamma}, 0\right)$, o tempo de flutuação de $\left(H_{t}, \bar{A}_{t}\right)_{t \geq 0} \mathrm{em}$ torno de $\left(h_{\gamma}, 0\right)$ tem distribuição de probabilidade próxima à distribuição exponencial, cujo valor esperado é $\gamma>0$. Isto ocorre, pois o tempo de flutuação de $\bar{A}_{t}$ em torno do excesso de demanda metaestável $\bar{A}_{+}\left(h_{\gamma}\right)\left(\bar{A}_{+}\left(h_{\gamma}\right)=0\right)$ é função de $h_{\gamma}$, que, neste caso, essencialmente não se altera com a atualização do preço, i.e., $H_{t_{k}^{(m)}+d t}=H_{t_{k}^{(m)}}+\lambda \bar{A}_{t_{k}^{(m)}} \simeq h_{\gamma}+\lambda 0=h_{\gamma}$. Fisicamente falando, o que mantém $H_{t}$ flutuando em torno de $h_{\gamma}$, por um tempo exponencial de parâmetro $\gamma$, é o excesso de oferta de fundamentalistas $(1-\alpha)(2 \Phi(-h)-1)$ que se sobrepõem ao excesso de procura 
metaestável de especuladores $\alpha \bar{S}_{+}(h)$ para qualquer sobrevalorização do ativo $h$ maior que $h_{\gamma}$, e vice-versa. Essa contraposição de forças estabelece um equilíbrio de mercado metaestável igualando oferta e procura $\left(\bar{A}\left(h_{\gamma}\right)=0\right)$ a um nível de sobrevalorização do ativo $h_{\gamma}$. Como $\bar{A}_{+}\left(h_{\gamma}\right)$ corresponde a um estado metaestável do sistema das configurações de atitudes de especuladores, i.e., $\bar{A}_{+}\left(h_{\gamma}\right)=\alpha \bar{S}_{+}\left(h_{\gamma}\right)+(1-\alpha)\left(2 \Phi\left(-h_{\gamma}\right)-1\right)$, onde $\bar{S}_{t} \simeq \bar{S}_{+}\left(h_{\gamma}\right)$, após um tempo exponencial de parâmetro $\gamma$, o sistema das configurações de atitudes de especuladores abandona seu estado metaestável e a magnetização empírica $\bar{S}_{t}$ abandona a magnetização metaestável em $\bar{S}_{+}\left(h_{\gamma}\right)$ e é atraída para a magnetização de equilíbrio do sistema, onde então $\bar{S}_{t}$ passa a flutuar em torno de $\lim _{S \rightarrow \mathbb{Z}^{2}} \mathbb{E}_{\mu_{S}}\left(X^{(0)} ; h_{\gamma}, \beta\right)$. Como isso, o excesso de demanda de equilíbrio resultante $\bar{A}_{t}$ passa a flutuar em torno do excesso de demanda de equilíbrio que, neste caso $\left(h_{\gamma}>0\right)$, é negativo, ou seja, $\bar{A}_{t} \simeq$ $\bar{A}_{-}\left(h_{\gamma}\right)=\bar{A}\left(h_{\gamma}\right)=\alpha \lim _{S \rightarrow \mathbb{Z}^{2}} \mathbb{E}_{\mu_{S}}\left(X^{(0)} ; h_{\gamma}, \beta\right)+(1-\alpha)\left(2 \Phi\left(-h_{\gamma}\right)-1\right)<0$. Como $\bar{A}_{t}$ flutua em torno do excesso de demanda de equilíbrio negativo, $H_{t}$ movimenta-se para a esquerda assim que a primeira atualização de preço ocorrer, lembrando que $H_{t_{k+1}^{(m)}}=H_{t_{k}^{(m)}}+\lambda \bar{A}_{t_{k}^{(m)}}$, $k=0,1,2 \ldots$ Essa movimentação de $H_{t}$ leva o sistema para o outro equilíbrio metaestável, onde então $\left(H_{t}, \bar{A}_{t}\right)$ passa a flutuar em torno de $\left(-h_{\gamma}, 0\right)$.

Lembrando que $P_{t}=H_{t}+\bar{P}$, temos que o preço $P_{t}$ tende a subir ou descer até alcançar um dos preços, $\bar{P}_{+}=\bar{P}+h_{\gamma}$ ou $\bar{P}_{-}=\bar{P}-h_{\gamma}$. Uma vez alcançado um destes preços, digamos $\bar{P}_{+}$, o preço $P_{t}$ passa a flutuar em torno de $\bar{P}_{+}$e o excesso de demanda $\bar{A}_{t}$, em torno do zero. O preço $\bar{P}_{+}$de fato satisfaz o conceito de preço de equilíbrio de mercado estável, no sentido que iguala oferta e procura de forma localmente estável. No entanto, após um tempo aleatório com distribuição aproximadamente exponencial, o preço do ativo abandona o nível $\bar{P}_{+}$e é atraído para $\bar{P}_{-}$, em torno do qual permanece flutuando por um tempo exponencial de igual parâmetro e assim por diante. Consideramos natural conceber estes preços, $\bar{P}_{+}$e $\bar{P}_{-}$, como sendo "preços metaestáveis de equilíbrio de mercado", unindo o conceito de metaestabilidade da mecânica estatística e o conceito de preço de equilíbrio de mercado da teoria econômica.

Por fim, gostaríamos de esclarecer em que sentido a escolha do parâmetro $\xi(1 / \xi$ é o tempo médio decorrido entre duas atualizações de preço) é compatível com um comportamento estratégico do market maker cuja liberdade de ação é a de ajustar o preço em intervalos de tempo de seu livre arbítrio. Do ponto de vista de um comportamento 
estratégico do market maker, é natural supor que o market maker opte por não reajustar o preço do ativo imediatamente após o último ajuste de preço efetuado. Ou seja, é natural supor que o market maker aguarde a reação do mercado e venha a reajustar o preço somente após ter julgado que o excesso de demanda tenha se estabilizado. No entanto, quando o excesso de demanda pertencer ao domínio de atração de um excesso de demanda metaestável $\left(\bar{A}_{t} \simeq \overline{(A}\right)_{+}\left(H_{t}\right), 0<H_{t}<h_{\gamma}$ ou $\left.\bar{A}_{t} \simeq \overline{(}(A)_{-}\left(H_{t}\right),-h_{0}<H_{t}<0\right)$, é igualmente natural supor que o market maker venha reajustar o preço assim que o excesso de demanda metaestável se estabeleça, i.e., muito antes que o excesso de demanda de equilíbrio seja atingido.

Isso ocorre devido à aparente estabilidade do excesso de demanda metaestável alcançado. Como julgamos que os tempos de espera necessários para que $\bar{A}_{t}$ atinja um excesso de demanda de equilíbrio ou excesso de demanda metaestável (partindo de seus respectivos domínios de atração) tenham, em média, a mesma ordem de grandeza $1 / \xi$, podemos então concluir que o market maker atualiza o preço em intervalos de tempo de duração média $1 / \xi$.

\subsubsection{Observações finais}

O modelo de interações locais entre especuladores que acabamos de apresentar carece de um tratamento mais rigoroso. Apresentamos aqui somente nossas conjecturas sobre o seu comportamento qualitativo e as principais idéias de como abordá-las.

Postulamos que a função de distribuição acumulada $\Phi$ possui uma forma paramétrica específica. Mediante esta forma paramétrica de $\Phi$, pudemos aplicar os conhecimentos sobre sistemas de spins, oriundos da teoria de Sistema de Partículas Interagentes, e remeter o processo das configurações de atitudes de investimentos de especuladores ao modelo de Ising ferromagnético estocástico. Neste contexto, a influência social direta $K$ emergiu como sendo diretamente proporcional à força de interação entre os spins; a subvalorização do ativo $-h$, igual ao campo externo; e a heterogeneidade das avaliações individuais sobre o valor fundamental do ativo $\left(\Phi^{\prime}(0)\right)^{-1}$, diretamente proporcional à temperatura. Essa abordagem permitiu conjecturar um comportamento do modelo que fosse análogo ao comportamento do modelo apresentado no Capítulo 2 (modelo de campo médio).

Não abordaremos o caso genérico, de interações globais e locais, no qual $J>0$ e $K>0$. 
Escolhemos o caso em que somente pode haver interações locais, $J=0$ e $K \geq 0$, pois este reúne os principais fenômenos que gostaríamos de focar: 1) existềncia de um único preço estável de equilíbrio de mercado igual à avaliação média sobre o valor fundamental do ativo; 2) existência de dois preços metaestáveis de equilíbrio de mercado; e 3) formação de bolhas especulativas e crashes de preço do ativo.

O tratamento do caso $J>0$ e $K>0$ implicaria na abordagem de interações globais e locais simultaneamente, cujo comportamento acreditamos não incorporar outros fenômenos além dos três já mencionados. Em última análise, acreditamos que o modelo que acabamos de apresentar tem o mesmo comportamento qualitativo que o modelo de campo médio apresentado no Capítulo 2. As implicações socioeconômicas são as mesmas já observadas naquele modelo. 


\section{Capítulo 5}

\section{Conclusão}

Apresentamos uma estrutura matemática baseada em sistemas de spins e usamos esta estrutura para construir três modelos sobre a dinâmica do preço de um ativo financeiro. Com base nos resultados obtidos, derivamos alguns fenômenos que podem ocorrer na vida real:

1. Existência de um único preço estável de equilíbrio de mercado do ativo igual à avaliação individual média sobre o valor fundamental do ativo. Esse fenômeno ocorre quando a fração de especuladores for suficientemente pequena e/ou quando a dispersão das avaliações individuais sobre o valor fundamental do ativo for relativamente alta, comparado com o grau com que especuladores procuram imitar o comportamento de outros agentes financeiros.

2. Existência de dois preços de equilíbrio de mercado localmente estáveis do ativo. Esse fenômeno ocorre quando a fração de especuladores for suficientemente positiva e pequena e/ou quando a dispersão das avaliações individuais sobre o valor fundamental do ativo for relativamente baixa, comparado com o grau com que especuladores procuram imitar o comportamento de outros agentes financeiros.

3. Formação de bolhas especulativas e crashes de preço do ativo. Esse fenômeno ocorre quando a fração de especuladores for suficientemente alta e/ou quando a dispersão das avaliações individuais sobre o valor fundamental do ativo for relativamente baixa, comparado com o grau com que especuladores procuram imitar o comportamento de outros agentes financeiros. 
Com base no primeiro modelo apresentado (modelo de campo médio), calculamos os seguintes valores críticos: dispersão crítica das avaliações individuais sobre o valor fundamental do ativo, preços de reversão de tendência (preços de crashes) e fração crítica de especuladores. Uma vez ajustado o modelo a dados reais, tais grandezas podem ser também de utilidade prática para a estimativa do risco de crash de preço de um ativo financeiro.

Pudemos também descrever como e quando uma intervenção no mercado pode ter um impacto positivo ou negativo sobre o equilíbrio de preço resultante. De acordo com o modelo, quando a dispersão das avaliações individuais sobre o valor fundamental do ativo for relativamente baixa, pequenas intervenções podem derrubar o preço de mercado do ativo, assim como gerar uma bolha especulativa (positiva).

Perspectivas futuras. Os modelos apresentados permitem várias outras investigações. Uma delas diz respeito ao fenômeno de "caldas pesadas" da função de densidade de probabilidades do retorno do ativo. Se, ao invés de estudarmos a evolução do preço $P_{t}$, estudarmos a distribuição limite do retorno, i.e., a distribuição de probabilidades de $\lim _{t \rightarrow \infty}\left(P_{t+1}-P_{t}\right)$, e considerarmos uma avaliação média sobre o valor fundamental do ativo não-fixa, mas seguindo um movimento Browniano $\bar{P}_{t}$ (modelando o impacto de notícias sobre a avaliação média sobre o valor fundamental do ativo ao longo do tempo), veremos, muito provavelmente, que as caldas da função de densidade de probabilidades de $\lim _{t \rightarrow \infty}\left(P_{t+1}-P_{t}\right)$, será tanto mais pesada quanto mais próxima da dispersão crítica estiver a dispersão das avaliações individuais sobre o valor fundamental do ativo. Isto, porque a derivada parcial do excesso de demanda de equilíbrio (magnetização do sistema) pela sobrevalorização do ativo (valor negativo do campo externo) tende ao infinito quando a dispersão das avaliações individuais sobre o valor fundamental do ativo (temperatura) tende à dispersão crítica (temperatura crítica). Ou seja, o excesso de demanda reage sensivelmente a pequenas oscilações da sobrevalorização do ativo. Este fato pode ser observado tanto no modelo de campo médio como no modelo de interações locais. Tal estudo poderia oferecer uma explicação para o fenômeno de "caldas pesadas" das funções de densidade de probabilidades de retornos de ativos financeiros, bem como indicar novos caminhos para a estimativa do risco de superdesvalorização de ativos financeiros. 
Quanto ao ajuste do modelo a dados reais, poderíamos iniciar a investigação com os dados provenientes do "livro de ordens" ( order book) de uma ativo financeiro. O livro de ordens é um arquivo que contém as últimas ordens de compra e venda do ativo. Neste arquivo estão listados: o tipo de ordem (compra ou venda), a quantidade e o preço para o qual o agente pretende fechar a transação. A partir do livro de ordens, podemos observar uma distribuição do volume de ordens de compra e venda em função do preço. Essa distribuição varia de ativo para ativo e também ao longo do tempo. Poderíamos iniciar alguns experimentos, ajustando a essa distribuição uma estimativa para o que chamamos em nosso modelo de "distribuição das avaliações individuais sobre o valor fundamental do ativo". 


\section{Capítulo 6}

\section{Apêndice}

Neste apêndice apresentaremos as provas das proposições 2 e 4 . -

\subsection{Demonstração da Proposição 2}

Proposição 2. Seja $\Phi$ uma função de distribuição de probabilidades que satisfaz as propriedades de (2.5). Sejam $\alpha \in[0,1], J \in[0, \infty)$ e $h \in \mathbb{R}$ três constantes arbitrárias e seja g como definida em (2.62). Definindo ${ }^{1} M\left(\alpha, J, \Phi^{\prime}(0)^{-1}, h\right):=\{x \in[-1,1] \mid x=$ $g(x ; h)\}$ e denotando o número de elementos de $M\left(\alpha, J, \Phi(0)^{-1}, h\right)$, quer dizer, o número de equilibrios do sistema (2.61), por $\left|M\left(\alpha, J, \Phi^{\prime}(0)^{-1}, h\right)\right|$, temos as seguintes relações:

1. Se $\left(\Phi^{\prime}(0)\right)^{-1} \geq 2 \alpha J$ então $\left|M\left(\alpha, J, \Phi^{\prime}(0)^{-1}, h\right)\right|=1$; e o único elemento de $M\left(\alpha, J, \Phi^{\prime}(0)^{-1}, h\right)$ é globalmente estável; mantendo os parâmetros $\alpha, J$ e $\Phi$ fixos, porém, arbitrários, e, denotando o único equilíbrio de $M\left(\alpha, J, \Phi^{\prime}(0)^{-1}, h\right)$ por $\bar{A}(h)$, temos que $\bar{A}(h)$ é estritamente decrescente, impar $\overline{(A}(0)=0$ ) e continuo em $h$ (veja a Figura 2.8).

2. $\operatorname{Se}\left(\Phi^{\prime}(0)\right)^{-1}<2 \alpha J$

então $\left|M\left(\alpha, J, \Phi^{\prime}(0)^{-1}, h\right)\right|$ pode ser 1,2 ou 3 , dependendo das condições expostas abaixo; estas condições envolvem o par de valores $\left(h_{*}, \bar{A}_{*}\right)>(0,0)$ chamados de

\footnotetext{
${ }^{1}$ A princípio, $M$ depende de todo $\Phi$; porém, as propriedades de $M$ que estão sendo estudadas na Proposição 2, dependem somente da característica $\left(\Phi^{\prime}(0)\right)^{-1}$ da função $\Phi$. É por isso que escrevemos $M$ como função desta característica e não de $\Phi$ como um todo.
} 
sobrevalorização crítica do ativo e excesso de demanda crítico do ativo, respectivamente, definidos pela solução do sistema abaixo que satisfaz $h_{*}>0$ e $\bar{A}_{*}>0$

$$
\left\{\begin{array}{l}
\bar{A}_{*}=\alpha\left[2 \Phi\left(J \bar{A}_{*}-h_{*}\right)-1\right]+(1-\alpha)\left[2 \Phi\left(-h_{*}\right)-1\right] \\
1=2 \alpha \Phi^{\prime}\left(J \bar{A}_{*}-h_{*}\right) J
\end{array}\right.
$$

(a) $S e-\infty<h<-h_{*}$

então $\left|M\left(\alpha, J, \Phi^{\prime}(0)^{-1}, h\right)\right|=1$ e o único elemento de $M\left(\alpha, J, \Phi^{\prime}(0)^{-1}, h\right)$, denotado por $\bar{A}_{+}(h)$, é um equilibrio globalmente estável;

(b) Se $h=-h_{*}$

então $\left|M\left(\alpha, J, \Phi^{\prime}(0)^{-1}, h\right)\right|=2$ e os dois elementos de $M\left(\alpha, J, \Phi^{\prime}(0)^{-1}, h\right)$, denotados por $\bar{A}_{-}\left(-h_{*}\right)$ e $\bar{A}_{+}\left(-h_{*}\right)$, satisfazem:

i. $\bar{A}_{-}\left(-h_{*}\right)<\bar{A}_{+}\left(-h_{*}\right) \operatorname{com} \bar{A}_{-}\left(-h_{*}\right)=-\bar{A}_{*}$;

ii. $\bar{A}_{-}\left(-h_{*}\right)$ é instável e $\bar{A}_{+}\left(-h_{*}\right)$ é localmente estável;

iii. $D\left(\bar{A}_{-}\left(-h_{*}\right)\right)=\left[-1,-\bar{A}_{*}\right]$ e $D\left(\bar{A}_{+}\left(-h_{*}\right)\right)=\left(-\bar{A}_{*}, 1\right]$

(domínios de atração dos equilíbrios);

(c) $S e-h_{*}<h<h_{*}$

então $\left|M\left(\alpha, J, \Phi^{\prime}(0)^{-1}, h\right)\right|=3$ e os três elementos de $M\left(\alpha, J, \Phi^{\prime}(0)^{-1}, h\right)$, denotados por $\bar{A}_{-}(h), \bar{A}(h)$ e $\bar{A}_{+}(h)$, satisfazem:

i. $\bar{A}_{-}(h)<-\bar{A}_{*}<\bar{A}(h)<\bar{A}_{*}<\bar{A}_{+}(h)$;

ii. $\bar{A}(h)$ é instável, enquanto que $\bar{A}_{-}(h)$ e $\bar{A}_{+}(h)$ são localmente estáveis;

iii. $D\left(\bar{A}_{-}(h)\right)=[-1, \bar{A}(h))$,

$$
\begin{aligned}
& D(\bar{A}(h))=\{\bar{A}(h)\}, \\
& D\left(\bar{A}_{+}(h)\right)=(\bar{A}(h), 1]
\end{aligned}
$$

(domínios de atração dos equilíbrios);

(d) $\operatorname{Se} h=h_{*}$

então $\left|M\left(\alpha, J, \Phi^{\prime}(0)^{-1}, h\right)\right|=2$ e os dois elementos de $M\left(\alpha, J, \Phi^{\prime}(0)^{-1}, h\right)$, denotados por $\bar{A}_{-}\left(h_{*}\right)$ e $\bar{A}_{+}\left(h_{*}\right)$, satisfazem:

i. $\bar{A}_{-}\left(h_{*}\right)<\bar{A}_{+}\left(h_{*}\right) \operatorname{com} \bar{A}_{+}\left(h_{*}\right)=\bar{A}_{*}$;

ii. $\bar{A}_{-}\left(h_{*}\right)$ é localmente estável e $\bar{A}_{+}\left(-h_{*}\right)$ é instável; 
iii. $D\left(\bar{A}_{-}\left(h_{*}\right)\right)=\left[-1, \bar{A}_{*}\right)$ e $D\left(\bar{A}_{+}\left(h_{*}\right)\right)=\left[\bar{A}_{*}, 1\right]$

(domínios de atração dos equilibrios);

(e) $S e h_{*}<h<\infty$

então $\left|M\left(\alpha, J, \Phi^{\prime}(0)^{-1}, h\right)\right|=1$ e o único elemento de $M\left(\alpha, J, \Phi^{\prime}(0)^{-1}, h\right)$, denotado por $\bar{A}_{-}(h)$, é um equilíbrio globalmente estável; $e$

(f) $\bar{A}_{+}(h)$ e $\bar{A}_{-}(h)$ são funções decrescentes e contínuas para $h$ pertencente a seus respectivos domínios de definição, i.e., para $h$ pertencente a $\left(-\infty, h_{*}\right]$ $e\left[-h_{*}, \infty\right)$, respectivamente. Além disso, vale $1=\bar{A}_{+}(-\infty)>\bar{A}_{+}(h) \geq \bar{A}_{*}>$ $0>-\bar{A}_{*} \geq \bar{A}_{-}(h)>\bar{A}_{-}(\infty)=-1, \forall h \in\left[-h_{*}, h_{*}\right]$ (veja a Figura 2.9).

Demonstração da Proposição 2. Vamos considerar o sistema dinâmico discreto

$$
\bar{A}_{t}=g\left(\bar{A}_{t-1} ; h\right), \quad t=0,1,2, \ldots
$$

onde

$$
g(\bar{A} ; h)=\alpha[2 \Phi(J \bar{A}-h)-1]+(1-\alpha)[2 \Phi(-h)-1]
$$

O objetivo aqui é estudar os equilíbrios de (6.1), bem como suas propriedades de estabilidade para diversas regiões dos parâmetros $J, \Phi^{\prime}(0), \alpha$ e $h$, tendo em vista que $\Phi$ satisfaz as propriedades estabelecidas em (2.5).

Seja $F(\bar{A} ; h):=g(\bar{A} ; h)-\bar{A}$. Os equilíbrios de (6.1) para cada $h$ correspondem às raízes de $F(\cdot, h)=0$, onde $F(\cdot, h)(x):=F(x ; h), \forall x \in[-1,1]$. É, entretanto, conveniente começar estudando as raízes de $F(\bar{A} ; \cdot)=0$, onde $F(\bar{A} ; \cdot)(y):=F(\bar{A} ; y), \forall y \in \mathbb{R}$. De fato, vamos mostrar inicialmente que existe uma única função $h(\bar{A}),-1<\bar{A}<1$, tal que $F(\bar{A} ; h(\bar{A}))=0$, e estudar suas propriedades.

Lema 3. Seja $F(\bar{A} ; h):[-1,1] \times \mathbb{R} \rightarrow \mathbb{R}$ definida por

$$
F(\bar{A} ; h)=g(\bar{A} ; h)-\bar{A}
$$

onde g é dada em (6.2). Para cada $-1<\bar{A}<1$, existe um único valor $h(\bar{A}) \in \mathbb{R}$, satisfazendo a equação $F(\bar{A} ; h(\bar{A}))=0$. A função $h(\bar{A})$, assim definida, é ímpar, derivável $e \lim _{\bar{A} \rightarrow \mp 1} h(\bar{A})= \pm \infty$. Além disso, vale: 
1. Se $\left(\Phi^{\prime}(0)\right)^{-1} \geq 2 \alpha J$, temos que

(a) $h(\bar{A})$ é estritamente decrescente.

(b) Seja, portanto, $\bar{A}(h)$ a função inversa de $h(\bar{A})$, então, segue de (1a):

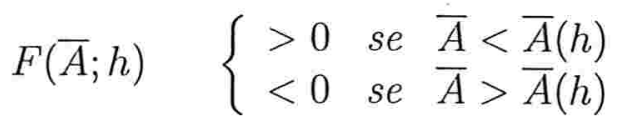

2. Se $\left(\Phi^{\prime}(0)\right)^{-1}<2 \alpha J$, temos que

(a) existe um único valor $\bar{A}_{*}$, tal que $0<\bar{A}_{*}<1, h^{\prime}\left(\bar{A}_{*}\right)=h^{\prime}\left(-\bar{A}_{*}\right)=0$ e

$$
h^{\prime}(A) \quad\left\{\begin{array}{lll}
<0 & \text { se } & \bar{A}<-\bar{A}_{*} \\
>0 & \text { se }-\bar{A}_{*}<\bar{A}<\bar{A}_{*} \\
<0 & \text { se } A>\bar{A}_{*}
\end{array}\right.
$$

(b) O sistema de equações para as variáveis $h$ e $\bar{A}$ dado por

$$
\left\{\begin{array}{l}
\bar{A}=\alpha[2 \Phi(J \bar{A}-h)-1]+(1-\alpha)[2 \Phi(-h)-1] \\
1=2 \alpha \Phi^{\prime}(J \bar{A}-h) J
\end{array}\right.
$$

admite somente as soluções $\left(h_{*}, \bar{A}_{*}\right)$ e $\left(-h_{*},-\bar{A}_{*}\right)$, onde $h_{*}:=h\left(\overline{A_{*}}\right)>0$, ou seja, $h_{*}>0$ e $\bar{A}_{*}>0$.

(c) Sejam, portanto, $\bar{A}_{-}(h), \bar{A}(h)$ e $\bar{A}_{+}(h)$ as funções inversas de $h(\bar{A})$ restritas a $\bar{A} \in\left(-1, \bar{A}_{*}\right], \bar{A} \in\left(-\bar{A}_{*}, \bar{A}_{*}\right)$ e $\bar{A} \in\left[\bar{A}_{*}, 1\right)$, respectivamente. Para estas funções, vale:

$\left.\bar{A}_{+}(h) \bar{A}_{-}(h)\right)$ é uma função estritamente decrescente e contínua em seu domínio de definição, i.e., em $\left(-\infty, h_{*}\right]\left(\left[-h_{*}, \infty\right)\right)$. $\bar{A}(h)$ é uma função estritamente crescente e contínua em seu domínio de definição, i.e., em $\left(-h_{*}, h_{*}\right)$. Além disso, $1=\bar{A}_{+}(-\infty)>\bar{A}_{+}(h) \geq \bar{A}_{*}>\bar{A}(h)>-\bar{A}_{*} \geq \bar{A}_{-}(h)>\bar{A}_{-}(\infty)=$ $-1, \forall h \in\left(-h_{*}, h_{*}\right)$.

Além disso, para $\forall h \in \mathbb{R}$, vale:

i. Se $|h|>h_{*}$, então $F(\cdot ; h)=0$ possui apenas uma raiz. Esta raiz é $\bar{A}_{+}(h)$ $\left.\bar{A}_{-}(h)\right)$ se $h<-h_{*}\left(h>h_{*}\right)$. Para a função $F(\cdot ; h)$ e para ambas as raízes, $\bar{A}_{+}(h)$ e $\bar{A}_{-}(h)$, representadas abaixo por $\bar{A}_{ \pm}(h)$, vale:

$$
F(\bar{A} ; h) \quad\left\{\begin{array}{lll}
>0 & \text { se } & \bar{A}<\bar{A}_{ \pm}(h) \\
<0 & \text { se } & \bar{A}>\bar{A}_{ \pm}(h)
\end{array}\right.
$$


ii. Se $h=-h_{*}$, então $F(\cdot ; h)=0$ possui exatamente duas raízes. Estas raízes são $\bar{A}_{-}(h)$ e $\bar{A}_{+}(h)$, onde

$$
F(\bar{A} ; h) \quad\left\{\begin{array}{lll}
<0 & \text { se } & \bar{A}>\bar{A}_{+}(h) \\
>0 & \text { se } & \bar{A}_{-}(h)<\bar{A}<\bar{A}_{+}(h) \\
>0 & \text { se } & \bar{A}<\bar{A}_{-}(h)
\end{array}\right.
$$

iii. Se $h=h_{*}$, então $F(\cdot ; h)=0$ possui exatamente duas raízes. Estas raízes são $\bar{A}_{-}(h)$ e $\bar{A}_{+}(h)$, onde

$$
F(\bar{A} ; h) \quad\left\{\begin{array}{lll}
<0 & \text { se } & \bar{A}>\bar{A}_{+}(h) \\
<0 & \text { se } & \bar{A}_{-}(h)<\bar{A}<\bar{A}_{+}(h) \\
>0 & \text { se } & \bar{A}<\bar{A}_{-}(h)
\end{array}\right.
$$

iv. Se $|h|<h_{*}$, então $F(\cdot ; h)=0$ possui exatamente três raízes. Estas raízes são $\bar{A}_{-}(h), \bar{A}(h)$ e $\bar{A}_{+}(h)$, onde

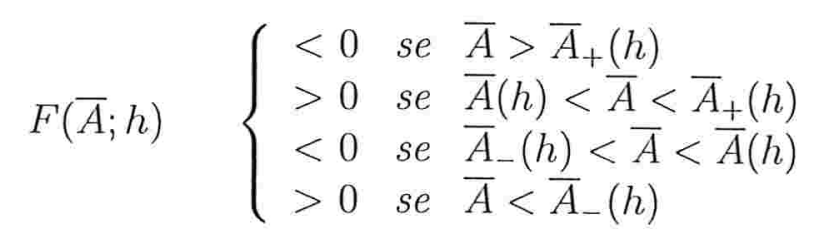

Demonstração do Lema. Observemos inicialmente que, para $-1<\bar{A}<1$, temos

$$
\lim _{h \rightarrow+\infty} F(\bar{A} ; h)=-1-\bar{A}<0<1-\bar{A}=\lim _{h \rightarrow-\infty} F(\bar{A} ; h)
$$

Assim, para cada $-1<\bar{A}<1$, a equação $F(\bar{A}, \cdot)=0$ possui pelo menos uma raiz. Observemos também que $F(\bar{A}, \cdot)$ é estritamente decrescente, isto é,

$$
\frac{\partial F(\bar{A} ; h)}{\partial h}=-\alpha\left[2 \Phi^{\prime}(J \bar{A}-h)\right]-(1-\alpha)\left[2 \Phi^{\prime}(-h)\right]<0, \quad \forall h \in \mathbb{R}
$$

De (6.10) e (6.11) obtemos a existência e unicidade de $h(\bar{A})$. Que $h(\bar{A})$ é ímpar, obtemos pelo seguinte argumento: como $2 \Phi-1$ é ímpar, $F$ definida em (6.3) é ímpar também, i.e., $F(-\bar{A},-h)=-F(\bar{A}, h), \forall(\bar{A}, h) \in[-1,1] \times \mathbb{R}$. Do fato de $F$ ser ímpar e da existência e unicidade de $h(\bar{A}), \forall \bar{A} \in(-1,1)$, segue

$$
F(-\bar{A},-h(\bar{A}))=-F(\bar{A}, h(\bar{A}))=0=F(-\bar{A}, h(-\bar{A})), \quad \forall \bar{A} \in(-1,1)
$$


De (6.12) e da unicidade da raiz de $F(\bar{A}, \cdot)=0$, segue então $h(-\bar{A})=-h(\bar{A}), \forall \bar{A} \in$ $(-1,1)$, o que mostra que $h(\bar{A})$ é ímpar.

Vamos mostrar agora que $\lim _{\bar{A} \rightarrow-1} h(\bar{A})=+\infty$. O argumento para $\bar{A} \rightarrow 1$ é análogo. Para tanto, temos que mostrar que, para qualquer $h \in \mathbb{R}$, existe $\varepsilon>0$, tal que

$$
h(\bar{A}) \geq h, \quad \forall \bar{A}:-1<\bar{A}<-1+\varepsilon
$$

Como $F(\bar{A} ; \cdot)$ é decrescente segundo (6.11), temos que a primeira desigualdade de (6.13) é equivalente a $F(\bar{A}, h(\bar{A})) \leq F(\bar{A}, h)$, onde, por definição, vale $F(\bar{A}, h(\bar{A}))=0$. Mostraremos portanto, que, para qualquer $h \in \mathbb{R}$, existe $\varepsilon>0$, tal que

$$
F(\bar{A}, h) \geq 0, \quad \forall \bar{A}:-1<\bar{A}<-1+\varepsilon
$$

Prova. Seja $\varepsilon:=\Phi(-J-h)$. Para todo $\bar{A}:-1<\bar{A}<-1+\varepsilon$, vale $\bar{A}<-1-\Phi(-J-h)<$ 0. Portanto, temos

$$
\begin{aligned}
F(\bar{A} ; h) & =\alpha[2 \Phi(J \bar{A}-h)-1]+(1-\alpha)[2 \Phi(-h)-1]-A \\
& \geq \alpha[2 \Phi(J \bar{A}-h)-1]+(1-\alpha)[2 \Phi(J \bar{A}-h)-1]-A \\
& =2 \Phi(J \bar{A}-h)-1-A \\
& \geq 2 \Phi(-J-h)-1-A \geq \Phi(-J-h) \geq 0
\end{aligned}
$$

A seguir, apresentaremos a derivada de $h(\bar{A})$ e mostraremos que $h^{\prime}(\bar{A})<J$. Tanto a expressão de $h^{\prime}(\bar{A})$, quanto a desigualdade $h^{\prime}(\bar{A})<J$ serão usadas na verificação dos itens 1 e 2 do Lema 3. Que $h(\bar{A})$ é diferenciável, segue do Teorema das Funções Implícitas. Calculando a derivada de $h(\bar{A})$, obtemos

$$
\begin{aligned}
h^{\prime}(A) & =\frac{\alpha 2 \Phi^{\prime}(J \bar{A}-h(\bar{A})) J-1}{\alpha 2 \Phi^{\prime}(J \bar{A}-h(\bar{A}))+(1-\alpha) 2 \Phi^{\prime}(-h(\bar{A}))} \\
& \leq J\left\{\frac{\alpha 2 \Phi^{\prime}(J \bar{A}-h(\bar{A}))}{\alpha 2 \Phi^{\prime}(J \bar{A}-h(\bar{A}))+(1-\alpha) 2 \Phi^{\prime}(-h(\bar{A}))}\right\}-\frac{1}{2 \Phi^{\prime}(0)} \\
& <J-\frac{1}{2 \Phi^{\prime}(0)}<J
\end{aligned}
$$

Demonstração do item 1 a do Lema 3. De $\left(\Phi^{\prime}(0)\right)^{-1}>2 \alpha J$ segue $\alpha\left[2 \Phi^{\prime}(0) J\right]-1<0$. Como $\Phi^{\prime}$ atinge seu valor máximo em 0 , segue que $2 \Phi^{\prime}(J \bar{A}-h(\bar{A})) J-1 \leq \alpha\left[2 \Phi^{\prime}(0) J\right]-1<0$, e, 


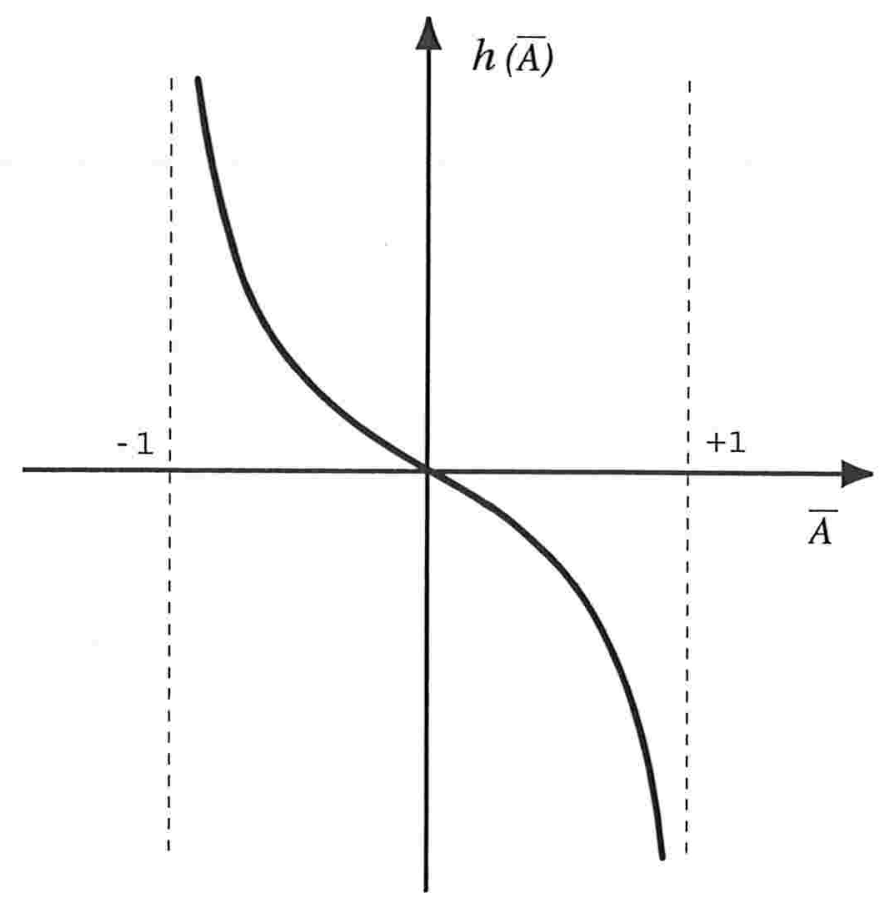

Figura 6.1: Caso $\left(\Phi^{\prime}(0)\right)^{-1} \geq 2 \alpha J$. Gráfico de $h(\bar{A})$.

portanto, $h^{\prime}(\bar{A})<0, \forall \bar{A} \in(-1,1)$. Isto é, $h(\bar{A})$ é estritamente decrescente (veja a Figura 6.1).

Como sugere a Figura 6.1, é fácil de ver também que $\left|h^{\prime}(\bar{A})\right|$ é estritamente decrescente para $\bar{A} \in(-1,0]$ e estritamente crescente para $\bar{A} \in[0,1)$. Essa propriedade de $h^{\prime}(\bar{A})$ segue do cálculo em (6.15) e das propriedades de $\Phi$ em (2.5).

Demonstração do item $1 b$ do Lema 3. Inicialmente, observemos que $F(-1, h)>0>$ $F(1, h), \forall h \in \mathbb{R}$. As duas últimas desigualdades seguem de $|2 \Phi(x)-1|<1, \forall x \in \mathbb{R}$. Como $h(\bar{A})$ é estritamente decrescente, $h(\bar{A})$ é inversível. Portanto, para qualquer $h \in \mathbb{R}$, a equação $F(\cdot ; h)=0$ possui uma única solução $\bar{A}(h)$. Uma vez que $\bar{A}(h)$ é a única solução de $F(\cdot ; h)=0$ e $F(-1, h)>0>F(1, h)$, segue $F(\bar{A}, h) \gtrless 0$ se $\bar{A} \lessgtr \bar{A}(h)$. Caso contrário, pelo Teorema do Valor Médio, teríamos uma outra solução de $F(\cdot ; h)=0$ à esquerda ou à direita de $\bar{A}(h)$.

Demonstração do item 2a do Lema 3. Como $2 \alpha \Phi^{\prime}(0) J-1>0$ e como a função de densidade de probabilidades $\Phi^{\prime}$ é simétrica em torno do zero e estritamente decrescente 
em $[0, \infty) \operatorname{com} \lim _{x \rightarrow \infty} \Phi^{\prime}(x)=0$, existe um único valor $a>0$, tal que

$$
2 \alpha \Phi^{\prime}(x) J-1 \quad\left\{\begin{array}{lll}
>0 & \text { se } & |x|<a \\
=0 & \text { se } & |x|=a \\
<0 & \text { se } & |x|>a
\end{array}\right.
$$

Agora, dada a desigualdade (6.16), a função $\varphi(\bar{A}):=J \bar{A}-h(\bar{A})$ é estritamente crescente, onde $\lim _{\bar{A} \rightarrow 1} \varphi(\bar{A})=\infty$. Além disso, $\varphi$ é ímpar, uma vez que $\varphi$ é uma combinação linear de funções ímpares. Portanto, segue de (6.17)

$$
2 \alpha \Phi^{\prime}(\varphi(\bar{A})) J-1 \quad\left\{\begin{array}{lll}
>0 & \text { se } & |\bar{A}|<\bar{A}_{*} \\
=0 & \text { se } & |\bar{A}|=\bar{A}_{*} \\
<0 & \text { se } & |\bar{A}|>\bar{A}_{*}
\end{array}\right.
$$

onde $\bar{A}_{*}$ é unicamente determinado por $\varphi\left(\bar{A}_{*}\right)=a$. Considerando que $2 \alpha \Phi^{\prime}(\varphi(\bar{A})) J-1$ é o numerador da fração do lado direito de (6.15), e que seu denominador é sempre positivo, segue

$$
h^{\prime}(\bar{A}) \quad\left\{\begin{array}{lll}
>0 & \text { se } & |\bar{A}|<\bar{A}_{*} \\
=0 & \text { se } & |\bar{A}|=\bar{A}_{*} \\
<0 & \text { se } & |\bar{A}|>\bar{A}_{*}
\end{array}\right.
$$

As desigualdades em (6.19) são as mesmas afirmadas no item 2a do Lema 3. A Figura (6.2) ilustra o gráfico de $h(\bar{A})$ no caso $\left(\Phi^{\prime}(0)\right)^{-1}<2 \alpha J$.

Demonstração do item $2 b$ do Lema 3. Vamos mostrar agora que $\left(h_{*}, \bar{A}_{*}\right)$ e $\left(-h_{*},-\bar{A}_{*}\right)$, $h_{*}:=h\left(\bar{A}_{*}\right)$, são as únicas soluções do sistema (6.5), sendo que $h_{*}>0$ e $\bar{A}_{*}>0$.

Inicialmente, motraremos que $\bar{A}_{*}>0$ e $h_{*}>0 . \bar{A}_{*}>0$ segue de $a>0$ em (6.17) e da monotonicidade de $\varphi$ definida logo após (6.17); agora, $h_{*}:=h\left(\bar{A}_{*}\right)>0$ segue de $h(0)=0$ e de $h^{\prime}(x)>0, \forall x: 0 \leq x \leq \overline{A_{*}}$.

Vamos mostrar agora que $\left(h_{*}, \bar{A}_{*}\right)$ é uma solução de (6.5). A segunda equação de (6.5) segue imediatamente de (6.18) e da definição de $\varphi$ apresentada logo após (6.18). A primeira equação de (6.5) segue da definição de $h_{*}$, isto é, como $h_{*}=h\left(\bar{A}_{*}\right)$, segue $F\left(\bar{A}_{*}, h_{*}\right)=0$. Esta última equação é equivalente à primeira equação de (6.5).

Mostraremos que $\left(h_{*}, \bar{A}_{*}\right)$ é a única solução "positiva" de $(6.5)$, i.e., onde $h_{*}>0$ e $\bar{A}_{*}>0$. Vamos mostrar que, para qualquer outra solução positiva $\left(h_{*}^{\prime}, \bar{A}_{*}^{\prime}\right)$, vale $\left(h_{*}, \bar{A}_{*}\right)=\left(h_{*}^{\prime}, \bar{A}_{*}^{\prime}\right)$. Pela segunda equação de $(6.5)$, segue $2 \alpha \Phi^{\prime}\left(\varphi\left(\bar{A}_{*}\right)\right) J=2 \alpha \Phi^{\prime}\left(\varphi\left(\bar{A}_{*}^{\prime}\right)\right) J$. 


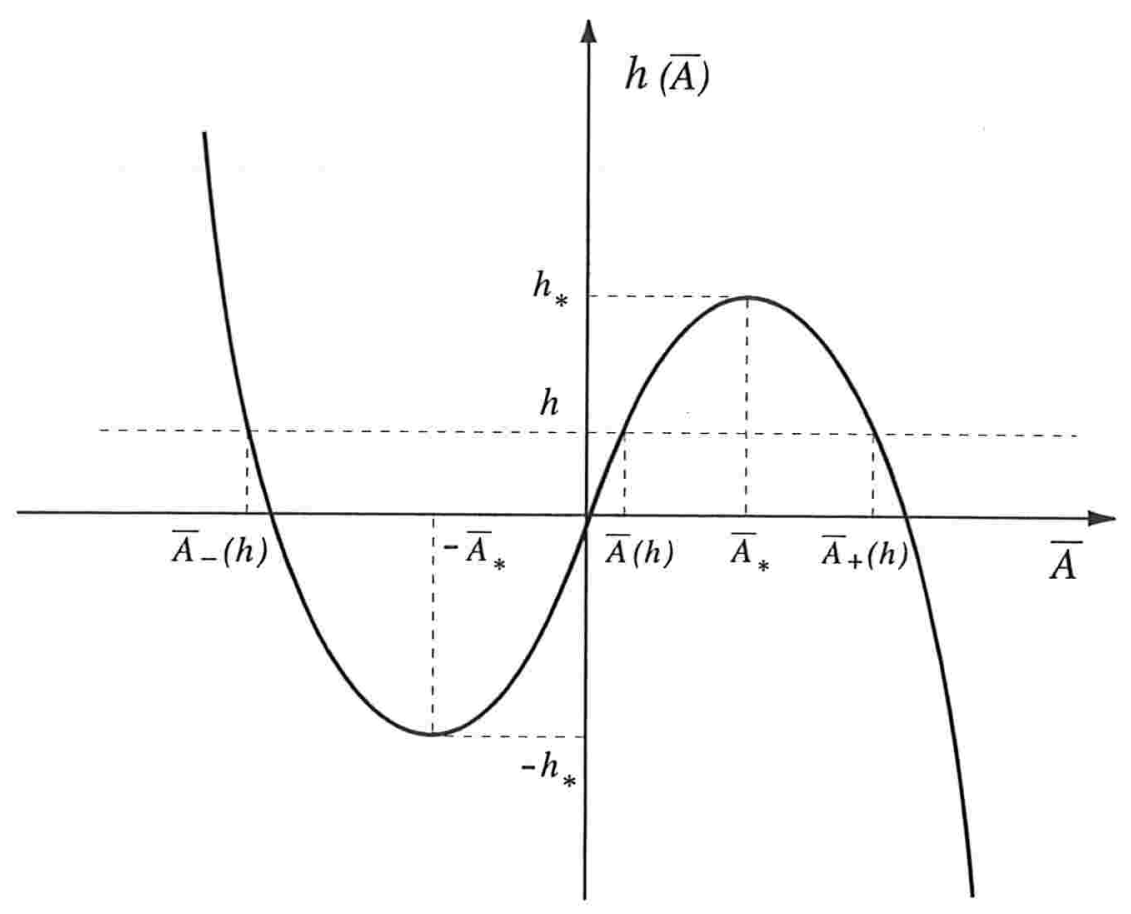

Figura 6.2: Caso $\left(\Phi^{\prime}(0)\right)^{-1}<2 \alpha J$. Gráfico de $h(\bar{A})$.

Como $\varphi$ é ímpar e estritamente crescente, e $\Phi^{\prime}$ é estritamente decrescente em [0,1], temos que $\Phi^{\prime}(\varphi(\cdot))$ é estritamente decrescente em $[0, \infty)$ também. Uma vez que $\bar{A}_{*}>0$ e $\bar{A}_{*}^{\prime}>0$, segue $\bar{A}_{*}=\bar{A}_{*}^{\prime}$. Agora, como para todo $\bar{A}$, existe um único $h(\bar{A})$ satisfazendo $F(\bar{A}, h(\bar{A}))=0$, segue, portanto, $h_{*}=h\left(\bar{A}_{*}\right)=h\left(\bar{A}_{*}^{\prime}\right)=h_{*}^{\prime}$.

Vamos mostrar agora que $\left(h_{*}, \bar{A}\right)$ e $\left(-h_{*},-\bar{A}\right)$ são as únicas soluções de (6.5). Como $\Phi^{\prime}(\varphi(\cdot)):[-1,1] \rightarrow \mathbb{R}$ é simétrica em torno do zero e estritamente decrescente em $[0,1]$ segue que $\left(h\left(-\bar{A}_{*}\right),-\bar{A}_{*}\right)$ também é solução da segunda equação de $(6.5)$, e, claro, não existe outra. Pela definição de $h\left(-\bar{A}_{*}\right)$, segue $F\left(h\left(-\bar{A}_{*}\right),-\bar{A}_{*}\right)=0$ que equivale à primeira equação de (6.5). Como $h(\cdot)$ é ímpar, segue que o sistema (6.5) possui somente as soluções $\left(h_{*}, \bar{A}\right)$ e $\left(-h_{*},-\bar{A}\right)=\left(-h\left(\bar{A}_{*}\right),-\bar{A}_{*}\right)$.

Demonstração do item $2 c$ do Lema 3. A monotonicidade das funções $\bar{A}_{-}(h), \bar{A}(h)$ e $\bar{A}_{+}(h)$ e suas localizações seguem imediatamente dos sinais da derivada de $h(A)$ em (6.19) e do fato de $h(\bar{A})$ ser uma função ímpar. A Figura 6.2 ilustra as localizações $\bar{A}_{-}(h), \bar{A}(h)$ e $\bar{A}_{+}(h)$ para um dado valor de $h$.

Provaremos a seguir os itens 2(c)i - 2(c)iv do Lema 3. Estes itens dizem respeito ao 
sinal de $F(\bar{A} ; h):=g(\bar{A} ; h)-\bar{A}$ para $(\bar{A}, h) \in[-1,1] \times \mathbb{R}$. Nos gráficos da Figura 2.5, que apresentamos na Seção 2.4.1.4, podemos reconhecer o sinal de $F(x, h)$ para $(x, h) \in$ $[-1,1] \times \mathbb{R}$; i.e., o valor de $F(x ; h)$ é positivo (negativo) quando o ponto $(g(x ; h), x)$ estiver acima (abaixo) da reta bissetriz do primeiro e terceiro quadrantes destes gráficos. Estas relações serão provadas abaixo.

Demonstração do item $2(c) i$ do Lema 3. Inicialmente, lembremos que sempre vale $F(-1, h)>$ $0>F(1, h), \forall h \in \mathbb{R}$. Isto porque $|2 \Phi(x)-1|<1, \forall x \in \mathbb{R}$.

Se $h>h_{*}\left(h<-h_{*}\right)$, segue das desigualdades estabelecidas em (6.19) que $\bar{A}_{-}(h)$ $\left(\bar{A}_{+}(h)\right)$ é a única raiz de $F(\cdot ; h)=0$. Como $F(\cdot ; h)=0$ possui uma única raiz e $F(-1, h)>0>F(1, h)$, seguem as desigualdades (6.6). Caso contrário, pelo Teorema do Valor Médio, existiria uma outra raiz à direita ou esquerda de $\bar{A}_{+}(h)\left(\bar{A}_{-}(h)\right)$.

Demonstração do item 2(c)ii do Lema 3. Se $h=-h_{*}$ segue, novamente de (6.19), que $\bar{A}_{-}(h)$ e $\bar{A}_{+}(h)$ são as únicas raízes de $F(\cdot ; h)=0$. Vamos mostrar inicialmente que $\partial F\left(\bar{A}_{+}(h) ; h\right) / \partial \bar{A}_{+}(h) \neq 0$.

Vamos supor, por contradição, $\partial F\left(\bar{A}_{+}(h) ; h\right) / \partial \bar{A}_{+}(h)=0$. Neste caso, $\partial g\left(\bar{A}_{+}(h) ; h\right) \partial \bar{A}_{+}(h)=$ 1. Como neste caso $\left(h, \bar{A}_{+}(h)\right)$ satisfaz a segunda equação de (6.5) e, pela definição de $\bar{A}_{+}(h)$, vale a primeira equação de $(6.5)$, i.e., $F\left(\bar{A}_{+}(h) ; h\right)=0$, segue que $\left(-h_{*}, \bar{A}_{*}\right)$ $\left(=\left(-h_{*}, \bar{A}_{+}\left(h_{*}\right)\right)\right.$ é solução de $(6.5)$. No entanto, já vimos com a verificação do item $2 \mathrm{~b}$ que o sistema $(6.5)$ admite somente as soluções $\left(h_{*}, \bar{A}_{*}\right)$ e $\left(-h_{*},-\bar{A}_{*}\right)$. Portanto, segue por contradição $\partial F\left(\bar{A}_{+}(h) ; h\right) / \partial \bar{A}_{+}(h) \neq 0$.

Agora, sendo que $F(1, h)<0, \forall h \in \mathbb{R}$, e, para as duas únicas raízes de $F(\cdot, h)=0$, vale $\bar{A}_{-}(h)<\bar{A}_{+}(h)$, segue imediatamente $\partial F\left(\bar{A}_{+}(h) ; h\right) / \partial \bar{A}_{+}(h)<0$ e $F(\bar{A}, h) \gtrless 0$ se $\bar{A}_{-}(h)<\bar{A} \lessgtr \bar{A}_{+}(h)$, caso contrário, pelo Teorema do Valor Médio, $F(\cdot, h)=0$ possuiria uma terceira raiz diferente de $\bar{A}_{-}(h)$ e $\bar{A}_{+}(h)$ localizada entre $\bar{A}_{-}(h)$ e 1 . Como $F(-1, h)>0$ e $\bar{A}_{-}(h)$ é a única raiz entre -1 e $\bar{A}_{-}(h)$, segue da mesma forma que $F(\bar{A}, h)>0$ se $\bar{A}<\bar{A}_{-}(h)$.

Os itens 2(c)iii e 2(c)iv, nos quais $h=-h_{*}$ e $|h|<h_{*}$, respectivamente, podem ser verificados argumentando de forma análoga. 
Acabamos de demonstrar o Lema 3. Vamos agora aplicar o Lema 3 e concluir a demonstração da Proposição 2.

Conclusão da Demonstração da Proposição 2. Os equilíbrios de (2.61) são as raízes de $F(\cdot ; h)=0, \operatorname{com} F(\bar{A} ; h)$ definida em (6.3). Portanto, as afirmações sobre o número e localização dos pontos de equilíbrio seguem imediatamente do Lema 3.

Vamos agora, então, estudar as propriedades de estabilidade desses equilíbrios. Observemos inicialmente que o gráfico de $g(\cdot ; h):[-1,1] \rightarrow[-1,1]$, onde $g(\cdot ; h)(\bar{A}):=$ $g(\bar{A} ; h)=\alpha[2 \Phi(J \bar{A}-h)-1]+(1-\alpha)[2 \Phi(-h)-1]$ (lado direito de (6.2)), pode ser obtido facilmente a partir do gráfico de $\Phi(x)$. De fato, $g(\cdot ; h)$ é obtida compondo a aplicação $x \mapsto 2 \Phi(x)-1$, com multiplicações e translações nas variáveis dependente e independente.

Os argumentos restantes da prova visam determinar o limite de $g^{(t)}\left(\bar{A}_{0} ; h\right)$ para os possíveis valores de $\bar{A}_{0}$ e $h$ em ambos os casos, $\left(\Phi^{\prime}(0)\right)^{-1} \geq 2 \alpha J$ e $\left(\Phi^{\prime}(0)\right)^{-1}<2 \alpha J$. Lembramos que $g^{(t)}\left(\bar{A}_{0} ; h\right)$ denota a $t$-ésima interação de $g(\cdot ; h)$ em $\bar{A}_{0}$, isto é,

$$
g^{(t)}\left(\bar{A}_{0} ; h\right)=g\left(g^{(t-1)}\left(\bar{A}_{0} ; h\right) ; h\right) \quad t=1,2, \ldots
$$

onde $g^{(1)}\left(\bar{A}_{0} ; h\right)=g\left(\bar{A}_{0} ; h\right)$.

As figuras 2.1 e 2.2, que apresentamos na Seção 2.4.1.3, ilustram o comportamento do sistema dinâmico $g^{(t)}\left(\bar{A}_{0} ; h\right)$ nos casos $\left(\Phi^{\prime}(0)\right)^{-1} \geq 2 \alpha J$ e $\left(\Phi^{\prime}(0)\right)^{-1}>2 \alpha J h<0$, respectivamente. No caso $\left(\Phi^{\prime}(0)\right)^{-1} \geq 2 \alpha J$ (Figura 2.1), o sistema dinâmico possui apenas um equilíbrio; no caso $\left(\Phi^{\prime}(0)\right)^{-1}<2 \alpha J$ (Figura 2.2), o sistema pode possuir um, dois ou três equilíbrios, dependendo do valor de $h$.

Vamos considerar primeiramente o caso $\left(\Phi^{\prime}(0)\right)^{-1} \geq 2 \alpha J$. Como já sabemos, neste caso $\left(\left(\Phi^{\prime}(0)\right)^{-1} \geq 2 \alpha J\right)$ existe um único ponto de equilíbrio $\bar{A}(h)$, para qualquer $h \in \mathbb{R}$.

Vamos provar que $\lim _{t \rightarrow \infty} g^{(t)}\left(\bar{A}_{0} ; h\right)=\bar{A}(h)$ para qualquer $\overline{A_{0}} \in[-1,1]$. Vamos supor inicialmente que $\bar{A}_{0} \geq \bar{A}(h)$. Observemos agora a seguinte implicação:

$$
\forall(h, \bar{A}) \in \mathbb{R} \times[-1,1]: \quad \bar{A} \geq \bar{A}(h) \Rightarrow \bar{A} \geq g(\bar{A} ; h) \geq \bar{A}(h)
$$

Provaremos (6.20) logo a seguir. Vamos supor que $\bar{A}_{0} \geq \bar{A}(h)$ e que a implicação (6.20) esteja satisfeita. Sendo assim, segue imediatamente por indução que a seqüência 
$g^{(t)}\left(\bar{A}_{0} ; h\right)$ será monótona, decrescente e inferiormente limitada por $\bar{A}(h)$. Portanto, $\lim _{t \rightarrow \infty} g^{(t)}\left(\bar{A}_{0} ; h\right)$ existe. Pela continuidade de $g(\cdot ; h)$, sabemos também que o limite é um ponto fixo, i.e., $\lim _{t \rightarrow \infty} g^{(t)}\left(\bar{A}_{0} ; h\right)=g\left(\lim _{t \rightarrow \infty} g^{(t-1)}\left(\bar{A}_{0} ; h\right) ; h\right)$. Como $\bar{A}(h)$ é o único ponto fixo, segue necessariamente $\lim _{t \rightarrow \infty} g^{(t)}\left(\bar{A}_{0} ; h\right) \downarrow \bar{A}(h)$.

Vamos agora verificar a validade de (6.20). Obtemos a segunda desigualdade do lado direito da implicação (6.20) aplicando $g(\cdot ; h)$ dos dois lados da desigualdade do lado esquerdo da implicação (6.20), onde levamos em conta que $g(\cdot ; h)$ é crescente e que $\bar{A}(h)$ é um ponto fixo de $g(\cdot ; h)$. Obtemos a primeira desigualdade do lado direito da implicação (6.20) pelo seguinte argumento: como, de acordo com o item 1b do Lema 3, vale $F(\bar{A}, h):=g(\bar{A}, h)-\bar{A}<0$ sempre que $\bar{A}>\bar{A}(h)$, segue $\bar{A}>g(\bar{A}, h)$ sempre que $\bar{A}>\bar{A}(h)$ e, claro, $\bar{A}=g(\bar{A}, h)$, sempre que $\bar{A}=\bar{A}(h)$.

Vamos supor agora $\bar{A}_{0}<\bar{A}(h)$. Neste caso, observamos que

$$
\forall(h, \bar{A}) \in \mathbb{R} \times[-1,1]: \quad \bar{A}<\bar{A}(h) \Rightarrow \bar{A}<g(\bar{A} ; h) \leq \bar{A}(h)
$$

Sob validade de $(6.21)$, segue novamente por indução que $g^{(t)}\left(\bar{A}_{0} ; h\right)$ é crescente e limitada superiormente por $\bar{A}(h)$. Novamente o limite existe e é ponto fixo. Uma vez que este limite é único, segue $g^{(t)}\left(\bar{A}_{0} ; h\right) \uparrow \bar{A}(h)$.

Verificaremos agora a validade de (6.21). A segunda desigualdade do lado direito da implicação de (6.21) segue igualmente do fato de $g(\cdot ; h)$ ser crescente. A primeira desigualdade do lado direito da implicação de (6.21) segue do item 1b do Lema 3, i.e., $F(\bar{A}, h):=g(\bar{A}, h)-\bar{A}>0$ sempre que $\bar{A}<\bar{A}(h)$.

Vamos considerar agora o caso $\left(\Phi^{\prime}(0)\right)^{-1}<2 \alpha J$. Como já sabemos, o número de equilíbrios de (6.1) depende neste momento do valor de $h$.

Se $|h|>h_{*}$, temos que os equilíbrios $\bar{A}_{+}(h)$ e $\bar{A}_{-}(h)$ são únicos em cada um dos subcasos $h<-h_{*}$ e $h>h_{*}$, respectivamente. Representando ambos os equilíbrios em cada um dos subcasos por $\bar{A}_{ \pm}(h)$, temos as desigualdades $g(\bar{A} ; h) \lessgtr \bar{A}$ se $\bar{A} \gtrless \bar{A}_{ \pm}(h)$ de (6.6). Como $g(\cdot ; h)$ é crescente, podemos argumentar da mesma forma como argumentamos no caso $\left(\Phi^{\prime}(0)\right)^{-1} \geq 2 \alpha J$ e mostrar a convergência monótona de $g^{(t)}\left(\bar{A}_{0} ; h\right)$ para $\bar{A}_{ \pm}(h)$, i.e., $g^{(t)}\left(\bar{A}_{0} ; h\right) \downarrow \bar{A}_{ \pm}(h)$ se $\bar{A}_{0} \geq \bar{A}_{ \pm}(h)$ e $g^{(t)}\left(\bar{A}_{0} ; h\right) \uparrow \bar{A}_{ \pm}(h)$ se $\bar{A}_{0}<\bar{A}_{ \pm}(h)$. 
Vamos considerar os casos $h=-h_{*}$ e $h=h_{*}$. Por simetria, basta que consideremos o caso $h=h_{*}$. Nesse caso, temos dois pontos de equilíbrio $\bar{A}_{-}(h)$ e $\bar{A}_{+}(h)$.

No caso $\bar{A}_{0} \geq \bar{A}_{+}(h)$, segue que $g^{(t)}\left(\bar{A}_{0} ; h\right)$ é decrescente e inferiormente limitada por $\bar{A}_{+}(h)$. Este fato segue de $g(\bar{A} ; h)<\bar{A} \Leftarrow \bar{A}_{+}(h)<\bar{A}$, assegurado por (6.8), argumentando da mesma forma como no caso $|h|>h_{*}$ e $\bar{A}_{0} \geq \bar{A}_{ \pm}(h)$, tratado anteriormente. Portanto, o limite existe, é superior ou igual a $\bar{A}_{+}(h)$ e é ponto fixo. Como não existem pontos fixos acima de $\bar{A}_{+}(h)$, segue $g^{(t)}\left(\bar{A}_{0} ; h\right) \downarrow \bar{A}_{+}(h)$.

No caso $\bar{A}_{-}(h) \leq \bar{A}_{0}<\bar{A}_{+}(h)$, segue que $g^{(t)}\left(\bar{A}_{0} ; h\right)$ é novamente decrescente e inferiormente limitada por $\bar{A}_{-}(h)$. Este fato segue de $g(\bar{A} ; h)<\bar{A} \Leftarrow \bar{A}_{-}(h)<\bar{A}<\bar{A}_{+}(h)$, assegurado igualmente por (6.8), argumentando da mesma forma como no caso $\bar{A}_{0} \geq \bar{A}_{+}(h)$ tratado acima. Portanto, o limite existe, satisfaz $\bar{A}_{-}(h) \leq \lim _{t \rightarrow \infty} g^{(t)}\left(\bar{A}_{0} ; h\right)<\bar{A}_{+}(h)$ e é ponto fixo. Como não existem pontos fixos abaixo de $\bar{A}_{-}(h)$, segue necessariamente $g^{(t)}\left(\bar{A}_{0} ; h\right) \downarrow \bar{A}_{-}(h)$.

No caso $\bar{A}_{0} \leq \bar{A}_{+}(h)$, segue que $g^{(t)}\left(\bar{A}_{0} ; h\right)$ é crescente e superiormente limitada por $\bar{A}_{-}(h)$. Este fato segue de $g(\bar{A} ; h)>\bar{A} \Leftarrow \bar{A} \leq \bar{A}_{-}(h)$, assegurado igualmente por (6.8), argumentando da mesma forma como no caso $|h|>h_{*}$ e $\bar{A}_{0} \leq \bar{A}_{ \pm}(h)$. Portanto, o limite existe, é inferior ou igual a $\bar{A}_{-}(h)$ e é ponto fixo. Como não existem pontos fixos abaixo de $\bar{A}_{-}(h)$, segue $g^{(t)}\left(\bar{A}_{0} ; h\right) \uparrow \bar{A}_{-}(h)$.

Finalmente, no caso $|h|<h_{*}$ temos três equilíbrios: $\bar{A}_{-}(h), \bar{A}(h)$ e $\bar{A}_{+}(h)$. Neste caso, temos: $g^{(t)}\left(\bar{A}_{0} ; h\right) \downarrow \bar{A}_{+}(h)$ se $\bar{A}_{0} \geq \bar{A}_{+}(h) ; g^{(t)}\left(\bar{A}_{0} ; h\right) \uparrow \bar{A}_{+}(h)$ se $\bar{A}(h)<\bar{A}_{0} \leq \bar{A}_{+}(h)$; $g^{(t)}\left(\bar{A}_{0} ; h\right) \downarrow \bar{A}_{+}(h)$ se $\bar{A}_{-}(h) \leq \bar{A}_{0}<\bar{A}(h)$; e $g^{(t)}\left(\bar{A}_{0} ; h\right) \uparrow \bar{A}_{+}(h)$ se $\bar{A}_{0} \leq \bar{A}_{-}(h)$. Estas convergências seguem aplicando um argumento análogo aos casos anteriores, levando em conta que $g(\cdot ; \bar{A})$ é crescente e levando em conta o sinal de $F(\bar{A} ; h):=g(\bar{A} ; h)-\bar{A}$ apresentado em (6.9), i.e., $g(\bar{A} ; h)<\bar{A}$ se $\bar{A}>\bar{A}_{+}(h) ; g(\bar{A} ; h)>\bar{A}$ se $\bar{A}(h)<\bar{A}<\bar{A}_{+}(h)$; $g(\bar{A} ; h)<\bar{A}$ se $\bar{A}_{-}(h)<\bar{A}<\bar{A}(h)$; e $g(\bar{A} ; h)>\bar{A}$ se $\bar{A}<\bar{A}_{-}(h)$.

Com a verificação deste último caso, fechamos a demonstração da Proposição 2. 


\subsection{Demonstração da Proposição 4}

Proposição 4. Seja $\left(H_{t}, \bar{A}_{t}\right)$ dado por

$$
\left\{\begin{array}{l}
H_{t}=H_{t-1}+\lambda \bar{A}_{t-1} \\
\bar{A}_{t}=\alpha\left[2 \Phi\left(J \bar{A}_{t-1}-H_{t}\right)-1\right]+(1-\alpha)\left[2 \Phi\left(-H_{t}\right)-1\right]
\end{array}\right.
$$

Então, $(0,0)$ é o único equilíbrio de $\left(H_{t}, A_{t}\right)_{t \geq 0}$. Além disso, existe um número positivo $\lambda_{c}$, tal que, para todo $\lambda$ com $0<\lambda<\lambda_{c}$, vale:

1. Se $\left(\Phi^{\prime}(0)\right)^{-1}>2 \alpha J$

então $(0,0)$ é um equilıbrio globalmente estável de $\left(H_{t}, \bar{A}_{t}\right)_{t \geq 0}$;

2. $\operatorname{Se}\left(\Phi^{\prime}(0)\right)^{-1}<2 \alpha J$

então $(0,0)$ é um equilibrio instável de $\left(H_{t}, A_{t}\right)_{t \geq 0}$ e para $\left(H_{0}, A_{0}\right) \neq(0,0)$ vale:

(a) $|\mathcal{T}|=\infty$;

(b) $\exists \tau_{\lambda} \in \mathbb{N}:\left|H_{\tau}\right|>h_{*} \forall \tau \in \mathcal{T} \operatorname{com} \tau>\tau_{\lambda}$,

onde $\mathcal{T}=\left\{\tau \in \mathbb{N} \mid \bar{A}_{\tau} \cdot \bar{A}_{\tau+1} \leq 0\right\}$ denota o conjunto dos tempos de reversão de sinal de $\left(\bar{A}_{t}\right)_{t \geq 0}$.

Demonstração. Dividiremos a demonstração em duas seções. Na seção seguinte apresentaremos a prova da Proposição 4 para o caso $\left(\Phi^{\prime}(0)\right)^{-1}>2 \alpha J$. Na seção posterior apresentaremos a prova para o caso $\left(\Phi^{\prime}(0)\right)^{-1}<2 \alpha J$. Em ambos os casos adotaremos novamente as seguintes notações: para cada $h \in \mathbb{R}$, denotamos com $g(\cdot, h)$ a função $g(\cdot, h):[-1,1] \rightarrow[-1,1]$, onde $g(\cdot, h)(\bar{A}):=g(\bar{A}, h)$; e para cada $\bar{A} \in[-1,1]$, denotamos com $g(\bar{A} ; \cdot)$ a função $g(\bar{A} ; \cdot): \mathbb{R} \rightarrow[-1,1]$, onde $g(\bar{A} ; \cdot)(h):=g(\bar{A}, h)$ e onde

$$
g(\bar{A}, h)=\alpha[2 \Phi(J \bar{A}-h)-1]+(1-\alpha)[2 \Phi(-h)-1]
$$

Das definições de $g(\cdot, h)$ e $g(\bar{A} ; \cdot)$ segue imediatamente que $g(\cdot, h)$ é crescente enquanto $g(\bar{A} ; \cdot)$ é decrescente, quaisquer que sejam $\bar{A} \in[-1,1]$ e $h \in \mathbb{R}$. Usaremos a monotonicidade de $g(\cdot, h)$ e $g(\bar{A} ; \cdot)$ no decorrer da demonstração. 


\subsubsection{Demonstração no caso $\left(\Phi^{\prime}(0)\right)^{-1}>2 \alpha J$}

Vamos mostrar inicialmente que $(0,0)$ é o único equilíbrio do sistema dinâmico $\left(H_{t}, \bar{A}_{t}\right)_{t \geq 0}$ dado por (6.22).

Demonstração. Seja $(H, \bar{A})$ um ponto de equilíbrio de (6.22). Da primeira equação de (6.22), segue que $\bar{A}=0$, caso contrário, pela condição de equilíbrio da primeira equação de (6.22), teríamos $H=H+\lambda \bar{A}=H+\lambda \bar{A} \neq H$. Substituindo $\bar{A}=0$ na segunda equação de (6.22), obtemos $2 \Phi(-\bar{H})-1=0$. Como $2 \Phi-1$ é ímpar, segue que $H=0$.

Vamos provar inicialmente a convergência de $\left(\bar{H}_{t}, \bar{A}_{t}\right)$ para $(0,0)$ no caso em que $J=0$ ou $\alpha=0$. Isto é, mostraremos que existe $\lambda_{c}>0$, tal que $\left(\bar{H}_{t}, \bar{A}_{t}\right) \rightarrow 0$ para todo $\lambda<\lambda_{c}$. Inicialmente, observemos que, se $J=0$ ou $\alpha=0$, o sistema dinâmico (6.22) adquire a forma

$$
\left\{\begin{array}{l}
H_{t}=H_{t-1}+\lambda \bar{A}_{t-1} \\
A_{t}=2 \Phi\left(-H_{t}\right)-1
\end{array}\right.
$$

Portanto, partindo de uma condição inicial $\left(H_{0}, \bar{A}_{0}\right)$, temos $H_{1}=H_{0}+\lambda \bar{A}_{0}$ e $\bar{A}_{1}=$ $2 \Phi\left(H_{1}\right)-1$, onde para $t \geq 2$, vale

$$
H_{t}=H_{t-1}+\lambda 2\left[\Phi\left(-H_{t-1}\right)-1\right]
$$

De (6.25) podemos derivar a seqüência $\left(\bar{A}_{t}\right)_{t \geq 0}$, uma vez que $\bar{A}_{t}=\left(H_{t+1}-H_{t}\right) / \lambda$, $\forall t \geq 1$. É claro que, se $H_{t}$ convergir para zero, $\bar{A}_{t}$ convergirá para zero também. Vamos provar, portanto, que a seqüência definida em (6.25) converge para zero.

Seja $f: \mathbb{R} \rightarrow \mathbb{R}$ dada por $f(x):=x+2 \lambda[\Phi(-x)-1]$. Como sua derivada $f^{\prime}(x)=$ $1-2 \lambda \Phi^{\prime}(-x)$ é simétrica em torno do zero, $f^{\prime}(x)$ restrito a $x \in[0, \infty)$ é estritamente crescente e como $\lim _{x \rightarrow 0} f^{\prime}(x)=1$, é fácil ver que a convergência de $H_{t}$, dada pela dinâmica $H_{t}=f\left(H_{t-1}\right)$, está garantida desde que $-1<f^{\prime}(0)$, isto é, desde que

$$
\lambda<\Phi^{\prime}(0)^{-1}
$$

Observação. É fácil ver que, se $\lambda>\Phi^{\prime}(0)^{-1}$, a seqüência $\left(H_{t}\right)_{t \geq 0}$ não converge para zero. Neste caso, a órbita de $\left(H_{t}\right)_{t \geq 0}$ se caracteriza por um "ciclo" em torno do 0 . É interessante observar que, mesmo sob ausência de atividade especulativa ( $\alpha=0$ ou 
$J=0$ ), se a heterogeneidade das avaliações individuais sobre o valor fundamental do ativo $\left(\Phi^{\prime}(0)\right)^{-1}$ for relativamente baixa em relação ao "ajuste do preço" $\lambda$, então $\lambda>\Phi^{\prime}(0)^{-1}$ e conseqüentemente $H_{t} \nrightarrow 0$.

Vamos agora considerar o subcaso $J>0$ e $\alpha>0$. Provaremos inicialmente cinco lemas que serão usados na prova da convergência de $\left(H_{t}, \bar{A}_{t}\right)$ para $(0,0)$ (Proposição 9). Para tanto, observemos que, se $g(\cdot ; h)=0$ possuir uma raiz, esta será única no caso que estamos considerando, i.e., no caso $\left(\Phi^{\prime}(0)\right)^{-1}>2 \alpha J$. Vamos adotar a seguinte notação: quando $g(\cdot ; h)=0$ possuir uma raiz, esta será denotada por $r(h)$, i.e.,

$$
g(r(h) ; h)=0
$$

Caso $g(\cdot ; h)=0$ não possua raiz, i.e., caso $g(x ; h)<0, \forall x \in \mathbb{R}$ ou $g(x ; h)>0, \forall x \in \mathbb{R}$, convencionaremos $r(h):=\infty$ se $g(x ; h)<0, \forall x \in \mathbb{R}$; e $r(h):=-\infty$ se $g(x ; h)>0$, $\forall x \in \mathbb{R}$.

Observação. Levando em conta a convenção acima estabelecida e o fato de que $g(\cdot ; h)$ é crescente, temos as seguintes relações para quaisquer $(\bar{A}, h) \in[-1,1] \times \mathbb{R}$ :

$$
g(\bar{A}, h) \leq 0 \text { se } \bar{A} \leq r(h) \quad \text { e } \quad g(\bar{A}, h) \geq 0 \text { se } \bar{A} \geq r(h)
$$

As relações em (6.27) serão usadas nos lemas que apresentaremos.

Precisamos, inicialmente, de estimativas para os valores de $r(h)$ e $\bar{A}(h)$. Lembramos que $\bar{A}(h)$ denota o ponto fixo de $g(\cdot, h)$, i.e., $\bar{A}(h)=g(\bar{A}(h) ; h)$. No Lema que segue apresentaremos estas estimativas.

Lema 4. Seja $b=2 \alpha \Phi^{\prime}(0)<1,0<a<b, h>0(h<0), M=\max \left\{\frac{1-\alpha}{\alpha} \frac{b}{a} h, h\right\}$. Suponhamos que $2 \alpha J \Phi^{\prime}(x) \geq a>0$ no intervalo $[-M, M]$. Então $\frac{1}{\alpha J} \frac{a}{b} h \leq r(h) \leq \frac{1}{\alpha J} \frac{b}{a} h$ $\left(-\frac{1}{\alpha J} \frac{b}{a} h \leq r(h) \leq-\frac{1}{\alpha J} \frac{a}{b} h\right)$.

Demonstração. Suporemos $h>0$. A prova para $h<0$ é análoga. Como $\frac{a}{\alpha J} \leq 2 \Phi^{\prime}(x) \leq \frac{b}{\alpha J}$ para $-h \leq x \leq h$, temos a seguinte estimativa para $\bar{F}:=(1-\alpha)[2 \Phi(-h)-1]$ :

$$
0 \leq \frac{1-\alpha}{\alpha J} a h \leq-\bar{F} \leq \frac{1-\alpha}{\alpha J} b h
$$


O gráfico de $g(\cdot ; \bar{A})$ passa pelo ponto $P=\left(\frac{h}{J}, \bar{F}\right)$. Sejam $r_{1}$ e $r_{2}$ as intersecções com o eixo das abscissas das retas que passam por $P$ com inclinação $b$ e $a$, respectivamente. É claro que $0<\frac{h}{J}<r_{1}<r_{2}$. Usando (6.28), obtemos

$$
\begin{aligned}
r_{2} & =\frac{-\bar{F}}{a}+\frac{h}{J} \leq \frac{1-\alpha}{\alpha J} \frac{b}{a} h+\frac{h}{J} \\
& =\frac{(1-\alpha) b+\alpha a}{\alpha J a} h \leq \frac{(1-\alpha) b+\alpha b}{\alpha J a} h=\frac{1}{\alpha J} \frac{b}{a} h \\
r_{1} & =\frac{-\bar{F}}{b}+\frac{h}{J} \geq \frac{1-\alpha}{\alpha J} \frac{a}{b} h+\frac{h}{J} \\
& =\frac{(1-\alpha) a+\alpha b}{\alpha J b} h \geq \frac{(1-\alpha) a+\alpha a}{\alpha J b} h=\frac{1}{\alpha J} \frac{a}{b} h
\end{aligned}
$$

Agora, como $a \leq 2 \alpha J \Phi^{\prime}(y) \leq b$ para $0 \leq y \leq \frac{1-\alpha}{\alpha} \frac{b}{a} h$, obtemos $a \leq g(\cdot ; h)^{\prime}(x) \leq b$ para $0 \leq J x-h \leq \frac{1-\alpha}{\alpha} \frac{b}{a} h$, ou seja,

$$
a \leq g(\cdot ; h)^{\prime}(x) \leq b, \quad \forall x: \frac{h}{J} \leq x \leq r_{2}
$$

Segue, então, que $r(h)$ está entre $r_{1}$ e $r_{2}$. Usando as desigualdades (6.29) e (6.30), obtemos, portanto, $\frac{1}{\alpha J} \frac{a}{b} h \leq r_{1} \leq r(h) \leq r_{2} \leq \frac{1}{\alpha J} \frac{b}{a} h$, como afirmado no lema (veja a Figura 6.3).

Observação. Dado qualquer valor $0<a<b$, temos $2 \alpha \Phi^{\prime}(x)>a$ para $|x| \leq \varepsilon$. Se $h$ é suficientemente pequeno (mais precisamente se $h$ é tal que $M \leq \varepsilon$ ), as hipóteses do Lema 4 se verificam.

Lema 5. Para $h>0$ (resp. $h<0)$, temos $\bar{A}(h) \geq \frac{-b}{J \alpha(1-b)} h\left(\right.$ resp. $\left.\bar{A}(h) \leq \frac{b}{J \alpha(1-b)} h\right)$.

Demonstração. Suporemos novamente $h>0$. Como já observamos durante a demonstração do Lema 4 , o gráfico de $g(\cdot ; h)$ passa pelo ponto $P=\left(\frac{h}{J}, \bar{F}\right)$. A reta que passa por $P$ e tem inclinação $b=2 \alpha J \Phi^{\prime}(0)$ é o gráfico da função $\Psi_{h}(x)=\bar{F}+b\left(x-\frac{h}{J}\right)$. Se $(\tilde{A}(h), \tilde{A}(h))$ denota a intersecção dessa reta com a bissetriz do primeiro e terceiro quadrantes, então, claramente, $\bar{A}(h) \geq \tilde{A}(h)$. Temos agora

$$
\Psi_{h}(\tilde{A}(h))=\tilde{A}(h) \Longleftrightarrow \tilde{A}(h)=\frac{\bar{F} J-b h}{J(1-b)}
$$




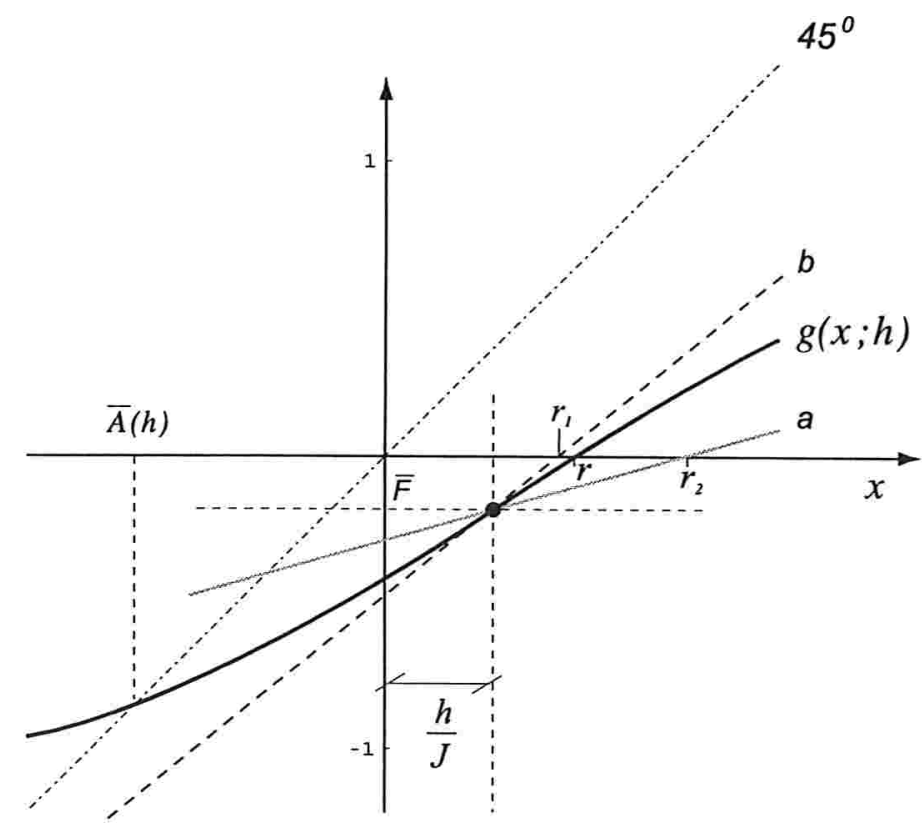

Figura 6.3: Caso $\left(\Phi^{\prime}(0)\right)^{-1}>2 \alpha J, h>0$. Gráfico de $g(\cdot ; h)$ e as raízes $r_{1}$ e $r_{2}$ das retas com inclinação $b$ e $a$ que passam pelo ponto $(h / J, \bar{F})$.

Da segunda desigualdade em (6.28) (que vale para qualquer valor de $h>0$ ) e de $\bar{A}(h) \geq \tilde{A}(h)$, segue então que

$$
\bar{A}(h) \geq \tilde{A}(h) \geq \frac{-\left(\frac{1-\alpha}{\alpha J} b h\right) J-b h}{J(1-b)}=-\frac{b}{J \alpha(1-b)} h
$$

provando o Lema.

Lema 6. Seja $\left(H_{t}, \bar{A}_{t}\right)_{n \geq 0}$ uma órbita do sistema dinâmico definido por (6.22). Então, ou $\left(H_{t}, \bar{A}_{t}\right) \rightarrow(0,0)$ ou existe $t \geq 0$, tal que $0 \leq H_{t+1} \leq \lambda \bar{A}_{t}$ ou $\lambda \bar{A}_{t} \leq H_{t+1} \leq 0$.

Demonstração. Suponhamos primeiro que $\bar{A}_{0}$ e $H_{0}$ tenham sinais distintos ou $\left(H_{0}, \bar{A}_{0}\right)=$ $(0,0)$ e, de fato, sem perda de generalidade,

$$
\bar{A}_{0} \leq 0 \leq H_{0}
$$

Vamos provar inicialmente que ou $\left(H_{t}, \bar{A}_{t}\right)$ converge para $(0,0)$ ou existe $t$, tal que $\lambda \bar{A}_{t} \leq H_{t+1} \leq 0$.

Demonstração. Basta provar que, se não existir $t \geq 0$, tal que $\lambda \bar{A}_{t} \leq H_{t+1} \leq 0$, então $\lim _{t \rightarrow \infty}\left(H_{t}, \bar{A}_{t}\right)=(0,0)$. Como $H_{t+1}-H_{t}=\lambda \bar{A}_{t}$, temos que $\lambda \bar{A}_{t} \leq H_{t+1} \leq 0 \Leftrightarrow H_{t+1} \leq$ 
$0 \leq H_{t}$. Agora, como por hipótese $0 \leq H_{0}$, segue que $0 \leq H_{t}, t=0,1,2, \ldots$, se não existir $t$, tal que $H_{t+1} \leq 0 \leq H_{t}$. Supondo que não exista tal $t$, temos $H_{t} \geq 0 \mathrm{e}$, conseqüentemente, $r\left(H_{t}\right) \geq 0, t=0,1,2, \ldots$ Usando $\bar{A}_{0} \leq r\left(H_{1}\right)$ e a monotonicidade de $g\left(\cdot ; H_{1}\right)$, segue imediatamente $\bar{A}_{1}=g\left(\bar{A}_{0} ; H_{1}\right) \leq g\left(r\left(H_{1}\right) ; H_{1}\right)=0$. Da mesma forma, concluímos: $\bar{A}_{t} \leq 0, t=2,3,4 \ldots$ Como $H_{t+1}=H_{t}+\lambda \bar{A}_{t}$ e $0 \leq H_{t}, t=0,1,2, \ldots$, segue imediatamente que $H_{t}$ é decrescente e limitada por zero. Portanto, $\bar{H}:=\lim _{t \rightarrow \infty} H_{t}$ existe. Deste fato segue imediatamente que $\bar{A}_{t}=\left(H_{t+1}-H_{t}\right) / \lambda \rightarrow 0$. Ou seja, $\left(H_{t}, \bar{A}_{t}\right)$ converge para $(\bar{H}, 0)$. Como o limite $(\bar{H}, 0)$ é um equilíbrio de $(6.22)$ e $(0,0)$ é único equilíbrio, obtemos $\lim _{t \rightarrow \infty}\left(H_{t}, \bar{A}_{t}\right)=(\bar{H}, 0)=(0,0)$.

Suponhamos agora que $\bar{A}_{0}$ e $H_{0}$ tenham o mesmo sinal. Em vista da simetria do problema, podemos supor, sem perda de generalidade:

$$
\bar{A}_{0} \geq 0 \quad \text { e } \quad H_{0} \geq 0
$$

Vamos mostrar que ou $\lim _{t \rightarrow \infty}\left(H_{t}, \bar{A}_{t}\right)=(0,0)$ ou existe $t$, tal que $\bar{A}_{t} \leq 0 \leq H_{t}$. Note que no último caso, i.e., caso exista um $t$, tal que $\bar{A}_{t} \leq 0 \leq H_{t}$, obtemos a situação (6.31); sob esta hipótese, o Lema já está provado.

Demonstração. Basta provar que, se não existir $t$, tal que $\bar{A}_{t} \leq 0 \leq H_{t}$, então $\lim _{t \rightarrow \infty}\left(H_{t}, \bar{A}_{t}\right)=$ $(0,0)$. Vamos supor que não exista tal $t$. Inicialmente, temos $H_{1}=H_{0}+\lambda \bar{A}_{0} \geq$ $H_{0} \geq 0$. Como o referido instante $t$ não existe, segue $\bar{A}_{1} \geq 0$. Da mesma forma, concluímos que $\bar{A}_{t} \geq 0, t=2,3, \ldots$ Deste fato segue imediatamente que $H_{t}$ é crescente. Se $\bar{H}:=\lim _{t \rightarrow \infty} H_{t}<\infty$, obtemos $\lim _{t \rightarrow \infty}\left(H_{t}, \bar{A}_{t}\right)=(\bar{H}, 0)=(0,0)$, como já concluímos no caso anterior. Mostraremos, por absurdo, que, se $\bar{H}=\infty$, então existe $t$, tal que $\bar{A}_{t} \leq 0 \leq H_{t}$. Como $H_{1} \geq 0$, segue que $\bar{A}\left(H_{1}\right) \leq 0$. Sendo também que $\left|\bar{A}_{1}-\bar{A}\left(H_{1}\right)\right|=\left|g\left(\bar{A}_{0} ; H_{1}\right)-\bar{A}\left(H_{1}\right)\right| \leq b\left|\bar{A}_{0}-\bar{A}\left(H_{1}\right)\right|$ e $\bar{A}\left(H_{1}\right) \leq 0 \leq \bar{A}_{0}$ e $\bar{A}\left(H_{1}\right) \leq 0 \leq \bar{A}_{1}$, obtemos $\bar{A}_{1} \leq \bar{A}_{0}$. Da mesma forma, obtemos $\bar{A}_{t+1} \leq \bar{A}_{t}, t=1,2, \ldots$, i.e., $\bar{A}_{t}$ é decrescente. Por outro lado, como $H_{t} \rightarrow \infty$, é fácil ver que $r\left(H_{t}\right) \rightarrow \infty$. Portanto, existe um primeiro instante $t \geq 0$ para o qual vale $\bar{A}_{t} \leq r\left(H_{t+1}\right)$. Para este instante, vale $\bar{A}_{t+1}=g\left(\bar{A}_{t} ; H_{t+1}\right) \leq g\left(r\left(H_{t+1}\right) ; H_{t+1}\right)=0 \leq H_{t+1}$, contrariando a hipótese da qual partimos.

Lema 7. Suponhamos $\lambda \leq \alpha J \frac{a}{b}$ e, tal que, se $h \leq \lambda$, as hipóteses do Lema 4 estejam satisfeitas. Suponhamos ainda $0 \leq H_{1}<\lambda \bar{A}_{0}$. Então, existe $t \geq 1$, tal que $0 \leq \bar{A}_{j} \leq$ 
$b \bar{A}_{j-1}, H_{j+1} \geq H_{j}, \bar{A}_{j-1} \geq r\left(H_{j}\right)$ para $j=1, \cdots, t$ e $\bar{A}_{t} \leq r\left(H_{t+1}\right)$.

Demonstração. Temos $\bar{A}_{0} \geq \frac{1}{\lambda} H_{1} \geq \frac{1}{\alpha J} \frac{b}{a} H_{1} \geq r\left(H_{1}\right)$, pelo Lema 4 (observe que as hipóteses do Lema 4 se verificam já que $\left.H_{1} \leq \lambda\right)$. Portanto, $\bar{A}_{1}=g\left(\bar{A}_{0} ; H_{1}\right)$ satisfaz $0 \leq \bar{A}_{1} \leq b \bar{A}_{0}$ e $H_{2}=H_{1}+\lambda \bar{A}_{1} \geq H_{1}$. Argumentando como no Lema 6 , concluímos que existe um valor mínimo de $t \geq 1$ para o qual $\bar{A}_{t} \leq r\left(H_{t+1}\right)$. Para $1 \leq j \leq t$, teremos $0 \leq \bar{A}_{j}=g\left(\bar{A}_{j-1} ; H_{j}\right) \leq b \bar{A}_{j}$ e $H_{j+1}=H_{j}+\lambda \bar{A}_{j} \geq H_{j}$.

Lema 8. Suponhamos $\lambda$ como no Lema 7 e $0 \leq \bar{A}_{0} \leq r\left(H_{1}\right)$. Então, ou existe $t \geq 1$, tal que $\bar{A}\left(H_{1}\right) \leq \bar{A}_{j} \leq 0$, para $j=1, \cdots, t ; 0 \leq H_{j} \leq H_{j-1}$ para $j=2, \cdots, t$; e $\lambda \bar{A}_{t} \leq H_{t+1} \leq 0$, ou $\left(H_{t}, \bar{A}_{t}\right) \rightarrow(0,0)$.

Demonstração. Observemos inicialmente que $H_{1} \geq 0$. Agora, como $\bar{A}\left(H_{1}\right) \leq 0 \leq \bar{A}_{0} \leq$ $r\left(H_{1}\right)$, segue da monotonicidade de $g\left(\cdot ; H_{1}\right)$ que $\bar{A}\left(H_{1}\right)=g\left(\bar{A}\left(H_{1}\right) ; H_{1}\right) \leq g\left(\bar{A}_{0} ; H_{1}\right)=$ $\bar{A}_{1} \leq g\left(r\left(H_{1}\right), H_{1}\right)=0$. Ou seja,

$$
\bar{A}\left(H_{1}\right) \leq \bar{A}_{1} \leq 0
$$

Agora, temos $H_{2}=H_{1}+\lambda \bar{A}_{1} \leq H_{1}$. Se $H_{2} \leq 0, \lambda \bar{A}_{1} \leq H_{1}+\lambda \bar{A}_{1}=H_{2} \leq 0$ e temos o resultado com $t=1$.

Caso contrário, i.e., caso $H_{2}>0, \bar{A}_{2}=g\left(\bar{A}_{1} ; H_{2}\right)$, satisfaz

$$
\left|\bar{A}_{2}-\bar{A}\left(H_{2}\right)\right| \leq b\left|\bar{A}_{1}-\bar{A}\left(H_{2}\right)\right|
$$

Além disso, como $0 \leq H_{2} \leq H_{1}$, temos

$$
\bar{A}\left(H_{1}\right) \leq \bar{A}\left(H_{2}\right)
$$

Se $\bar{A}_{1} \leq \bar{A}\left(H_{2}\right)$, temos pela monotonicidade $g\left(\cdot ; H_{2}\right)$ que $\bar{A}_{2}=g\left(\bar{A}_{1} ; H_{2}\right) \leq g\left(\bar{A}\left(H_{2}\right) ; H_{2}\right)=$ $\bar{A}\left(H_{2}\right)$. Como, então, $\bar{A}_{1} \leq \bar{A}\left(H_{2}\right)$ e $\bar{A}_{2} \leq \bar{A}\left(H_{2}\right)$, obtemos de (6.34) $\bar{A}_{1} \leq \bar{A}_{2}$. Da primeira desigualdade de (6.33) segue, portanto, $\bar{A}\left(H_{1}\right) \leq \bar{A}_{2}$.

Se $\bar{A}\left(H_{2}\right) \leq \bar{A}_{1}$, então, devido a (6.35) e pela monotonicidade de $g\left(\cdot ; H_{2}\right)$, temos $\bar{A}\left(H_{1}\right) \leq$ $\bar{A}\left(H_{2}\right)=g\left(\bar{A}\left(H_{2}\right) ; H_{2}\right) \leq g\left(\bar{A}_{1} ; H_{2}\right)=\bar{A}_{2}$.

Em qualquer caso temos, portanto, $\bar{A}\left(H_{1}\right) \leq \bar{A}_{2}$. Além disso, como $\bar{A}_{1} \leq 0 \leq H_{2}$, temos $\bar{A}_{1} \leq 0 \leq r\left(H_{2}\right)$ e, portanto, $\bar{A}_{2}=g\left(\bar{A}_{1} ; H_{2}\right) \leq 0$. 
Agora, $\lambda \bar{A}_{2} \leq H_{3}=H_{2}+\lambda \bar{A}_{2} \leq H_{2}$. Se $H_{3} \leq 0$, temos o resultado com $t=2$.

Prosseguindo desta maneira, se após $k$ passos obtivermos $H_{k+1} \leq 0$, teremos o resultado com $t=k$. Caso contrário, $H_{t}$ é decrescente e limitada inferiormente por zero. Neste caso, o limite $\bar{H}:=\lim _{t \rightarrow 0} H_{t}$ existe e, como já vimos no Lema anterior, segue deste fato: $\lim _{t \rightarrow 0}\left(H_{t}, \bar{A}_{t}\right)=(\bar{H}, 0)=(0,0)$, o que prova o Lema.

Estamos agora em condições de provar a primeira parte da Proposição 4 no caso $J>0$ e $\alpha>0$. A Proposição 9, que apresentaremos a seguir, resulta na prova da primeira parte da Proposição 4 no caso $J>0$ e $\alpha>0$. Esclareceremos esta implicação após a demonstração da Proposição 9.

Proposição 9. Seja $k \geq 1$ número inteiro, tal que $b^{k} \leq \frac{1}{4} \frac{\alpha(1-b)}{b}$. Suponhamos que $\lambda$ satisfaz as condições do Lema 7 , e tal que $\lambda k \leq \frac{1}{4} \frac{J \alpha(1-b)}{b}$. Então, para toda condição inicial $\left(\bar{A}_{0}, H_{0}\right)$, temos $\left(\bar{A}_{t}, H_{t}\right) \rightarrow(0,0)$.

Demonstração. Pelo Lema 6, podemos supor, sem perda de generalidade, $\lambda \bar{A}_{0} \geq H_{1} \geq 0$. Seja $t \geq 1$, dado pelo Lema 7 .

Se $(t-1) \geq k$, então

$$
H_{t} \leq J r\left(H_{t}\right) \leq J \bar{A}_{t-1} \leq J b^{k} \bar{A}_{0} \leq J \frac{1}{4} \frac{\alpha(1-b)}{b} \bar{A}_{0}
$$

A primeira desigualdade acima vem de $H_{t} / J \leq r\left(H_{t}\right)$, uma vez que $H_{t} \geq 0$ para $t$ escolhido segundo o Lema 7. A segunda desigualdade acima segue de $0 \leq \bar{A}_{j} \leq b \bar{A}_{j-1}, j=1, \ldots t$ segundo o Lema 7 e a última desigualdade segue do enunciado desta demonstração. Por outro lado, se $(t-1)<k$, então

$$
\begin{aligned}
H_{t} \leq H_{t}+\left(\lambda \overline{A_{0}}-H_{1}\right) & =H_{1}+\lambda \sum_{j=1}^{t-1} \bar{A}_{j}+\left(\lambda \overline{A_{0}}-H_{1}\right) \\
& =\lambda \sum_{j=0}^{t-1} \bar{A}_{j} \leq \lambda k \bar{A}_{0} \leq J \frac{1}{4} \frac{\alpha(1-b)}{b} \bar{A}_{0}
\end{aligned}
$$

A desigualdade de (6.37) segue da condição inicial $\lambda \bar{A}_{0} \geq H_{1} \geq 0$ que assumimos com base no Lema 6. A primeira desigualdade de (6.38) segue de $k \geq n$ e, segundo o Lema 7, de $\bar{A}_{j} \leq \bar{A}_{0}$ para $j=1,2, \ldots$. A última desigualdade de (6.38) segue do enunciado desta demonstração. 
Portanto, em ambos os casos, vale

$$
H_{t} \leq J \frac{1}{4} \frac{\alpha(1-b)}{b} \bar{A}_{0}
$$

Como $\overline{A_{t}} \leq \overline{A_{0}}$ e $\lambda \leq k \lambda$, temos

$$
H_{t+1}=H_{t}+\lambda \bar{A}_{t} \leq H_{t}+k \lambda \bar{A}_{0} \leq J \frac{1}{2} \frac{\alpha(1-b)}{b} \bar{A}_{0}
$$

e, usando o Lema 5, segue

$$
\bar{A}\left(H_{t+1}\right) \geq-\frac{b}{J \alpha(1-b)} H_{t+1} \geq-\frac{1}{2} \bar{A}_{0}
$$

Agora, pela escolha de $t$ segundo o Lema 7 , temos $0 \leq \bar{A}_{t} \leq r\left(H_{t+1}\right)$. As duas últimas desigualdades satisfazem a hipótese do Lema 8. Pelo Lema 8, ou $\left(H_{t}, \bar{A}_{t}\right) \rightarrow(0,0)$ ou existe $m \geq 1$, tal que $\bar{A}\left(H_{t+1}\right) \leq \bar{A}_{j} \leq 0$, para $j=t+1, \cdots, t+m, 0 \leq H_{j} \leq H_{j-1}$ para $j=t+$ $2, \cdots, t+m$ e $\lambda \bar{A}_{t+m} \leq H_{t+m+1} \leq 0$. Portanto, a seqüência $\bar{A}_{0}, \bar{A}_{1}, \cdots, \bar{A}_{t}, \bar{A}_{t+1}, \cdots, \bar{A}_{t+m}$ possui a seguinte propriedade: $\bar{A}_{0}, \bar{A}_{1}, \cdots, \bar{A}_{t}$ é decrescente pelo Lema 7 e $\bar{A}_{t+1}, \cdots, \bar{A}_{t+m}$ são maiores ou iguais a $-\frac{1}{2} \bar{A}_{0}$, uma vez que estes termos são maiores ou iguais a $\bar{A}\left(H_{t+1}\right)$ $\left(\geq-\frac{1}{2} \bar{A}_{0}\right)$ pelo Lema 8 .

Como $\lambda \bar{A}_{t+m} \leq H_{t+m+1} \leq 0$, o argumento acima pode ser repetido, tomando $\bar{A}_{t+m}$ e $H_{t+m+1}$ como condição inicial. Obteremos assim uma sequência $\bar{A}_{t+m+1}, \bar{A}_{t+m+2}$, $\cdots, \bar{A}_{t+m+l}, \cdots \bar{A}_{t+m+l+1}, \bar{A}_{t+m+l+2}, \cdots, \bar{A}_{t+m+l+s}$, com $l, s \geq 1$, tal que $\bar{A}_{t+m+1} \leq$ $\bar{A}_{t+m+2} \leq \bar{A}_{t+m+l} \leq 0$ e $0 \leq \bar{A}_{t+m+l+1}, \bar{A}_{t+m+2}, \cdots, \bar{A}_{t+m+l+s} \leq-\frac{1}{2} \bar{A}_{t+m} \leq\left(\frac{1}{2}\right)^{2} \bar{A}_{0}$. O mesmo argumento pode ser repetido indefinidamente e concluímos que $\bar{A}_{t} \rightarrow 0$.

Mostraremos agora que $H_{t} \rightarrow 0$. Vamos supor que $H_{t} \nrightarrow 0$. Então, existe $\varepsilon>0$ e uma subsequiência $\left(t_{k}\right)_{k \geq 0}$, tal que $H_{t_{k}}>\varepsilon, \forall k \geq 0$ ou $H_{t_{k}}<-\varepsilon, \forall k \geq 0$. Vamos supor, sem perda de generalidade, que $H_{t_{k}}>\varepsilon, \forall k \geq 0$. Como $g(x ; h)=\alpha(2 \Phi(J x-h)-1)+(1-$ $\alpha)(2 \Phi(-h)-1)$ é decrescente em $h$, segue imediatamente que

$$
\bar{A}_{t_{k}}=g\left(\bar{A}_{t_{k}-1} ; H_{t_{k}}\right) \leq g\left(\bar{A}_{t_{k}-1} ; \varepsilon\right)
$$

Como $\bar{A}_{t} \rightarrow 0$ e $g(\cdot ;-\varepsilon)$ é contínua, podemos passar o limite dos dois lados da desigualdade acima obtendo $0 \leq g(0 ;-\varepsilon)=2 \Phi(-\varepsilon)-1<0$. Posto que a última desigualdade é um absurdo, segue $\bar{A}_{t} \rightarrow 0$ e, portanto, $\left(H_{t}, \bar{A}_{t}\right) \rightarrow(0,0)$. 
Observação. Esta demonstração impõe uma série de condições implícitas sobre $\lambda$. Estas condições serão reunidas agora. Destas condições segue a existência de um $\lambda_{c}$ para o caso $J>0$ e $\alpha>0$, tal que $\left(H_{t}, \bar{A}_{t}\right) \rightarrow(0,0)$ sempre que $\lambda<\lambda_{c}$.

Sejam $a<b:=2 \alpha \Phi^{\prime}(0) J$ e $k \geq 1$, tal que $b^{k} \leq \frac{1}{4} \frac{\alpha(1-b)}{b}$. Basta escolher $\lambda_{c}>0$, tal que valha simultaneamente:

1. $\lambda_{c} k<\frac{1}{4} \frac{J \alpha(1-b)}{b}$;

2. $\lambda_{c}<\alpha J \frac{a}{b} ; \mathrm{e}$

3. $2 \alpha \Phi^{\prime}\left(\operatorname{Max}\left\{\frac{1-\alpha}{\alpha} \frac{b}{a} h, h\right\}\right)>a$ para qualque $h$ satisfazendo $0 \leq h<\lambda_{c}$.

A primeira condição vem do enunciado da Proposição 9. A segunda condição vem do Lema 7 e a terceira condição, da Observação que segue o Lema 4. É claro que existe $\lambda_{c}>0$ e que as condições acima também estão satisfeitas para todo $\lambda \leq \lambda_{c}$. Resta saber para qual escolha de $a$ e $k$ podemos encontrar $\lambda_{c}$ máximo. Este tema não será abordado.

Com a Proposição 9 e a última observação acima, fechamos a demonstração da Proposição 4 no caso $\left(\Phi^{\prime}(0)\right)^{-1}>2 \alpha J$. 


\subsubsection{Demonstração no caso $\left(\Phi^{\prime}(0)\right)^{-1}<2 \alpha J$}

A presente seção destina-se à demonstração das afirmações 2(a) e 2(b) da Proposição 4. Lembramos que 2(a) afirma que, sob certas condições, $|\mathcal{T}|=\infty$, onde $\mathcal{T}:=\{\tau \in$ $\left.\mathbb{N}: \bar{A}_{\tau} \cdot \bar{A}_{\tau+1}<0\right\}$ denota o conjunto dos tempos de reversão de sinal da seqüência $\left(\bar{A}_{t}\right)_{t \geq 0}$, enquanto 2(b) afirma que, sob certas condições, $\left|h_{\tau}\right|>h_{*}$ para todo $\tau$ suficientemente grande. Lembramos ainda que $\left(\bar{A}_{t}\right)_{t \geq 0}$ e $\left(H_{t}\right)_{t \geq 0}$ são as seqüências "componentes" da seqüência $\left(H_{t}, \bar{A}_{t}\right)_{t \geq 0}$, que está determinada, a partir de uma dada condição inicial $\left(H_{0}, \bar{A}_{0}\right)$, pelo sistema $(6.22)$.

Nos argumentos da presente demonstração usaremos as constantes $h_{*}$ e $\bar{A}_{*}$, onde a constante $h_{*}$ aparece no enunciado da Proposição 4. Estas constantes são positivas e unicamente determinadas pelo sistema

$$
\left\{\begin{array}{l}
\bar{A}_{*}=\alpha\left[2 \Phi\left(J \bar{A}_{*}-h_{*}\right)-1\right]+(1-\alpha)\left[2 \Phi\left(-h_{*}\right)-1\right] \\
1=2 \alpha \Phi^{\prime}\left(J \bar{A}_{*}-h_{*}\right) J
\end{array}\right.
$$

Achamos útil lembrar que a interpretação geométrica das constantes positivas $h_{*}$ e $\bar{A}_{*}$ é a seguinte: $h_{*}$ é o valor de $h$, para o qual o gráfico da função $g(\cdot ; h)$ tangencia a semi-reta bissetriz do primeiro quadrante; $\bar{A}_{*}$ é o valor do argumento de $g\left(\cdot ; h_{*}\right)$ para o qual ocorre essa tangência (veja a Figura 2.5, subcaso d).

A demonstração das afirmações 2(a) e 2(b) da Proposição 4 para as condições iniciais em que $\left|\bar{A}_{0}\right| \geq \bar{A}_{*}$ segue do Lema 9 apresentado a seguir. Para as condições iniciais em que $\left|\bar{A}_{0}\right|<0$ e $\left(\bar{A}_{0}, H_{0}\right) \neq(0,0)$, usamos adicionalmente a conjectura apresentada adiante.

Lema 9. Existe $\lambda_{c}>0$ (cujo valor exato depende dos parâmetros do modelo), tal que, caso $\lambda \in\left(0 ; \lambda_{c}\right],\left(\Phi^{\prime}(0)\right)^{-1}<2 \alpha J$ e $\bar{A}_{0} \geq \bar{A}_{*}$ existem $u$ e $v, u \leq v<\infty$, tais que o processo $\left(H_{t}, \bar{A}_{t}\right)_{t \geq 0}$ possui as seguintes propriedades:

$$
\begin{gathered}
\bar{A}_{0}>0, \ldots, \bar{A}_{u-1}>0, \bar{A}_{u} \leq 0, \bar{A}_{u+1}<0, \ldots, \bar{A}_{v-1}<0, \bar{A}_{v} \leq-\bar{A}_{*} \\
e \\
H_{u}>h_{*}
\end{gathered}
$$

Conjectura. Existe $\lambda_{c}>0$, tal que para qualquer que seja a condição inicial $\left(H_{0}, \bar{A}_{0}\right) \neq$ $(0,0)$ e $\lambda<\lambda_{c}$, existe um tempo finito $\tau_{\lambda}$, tal que

$$
\operatorname{Max}\left\{\left|\bar{A}_{0}\right|,\left|\bar{A}_{1}\right|,\left|\bar{A}_{2}\right|, \ldots\left|\bar{A}_{\tau_{\lambda}}\right|\right\} \geq \bar{A}_{*}
$$


A conjectura é intuitiva. Além disso, temos também evidências computacionais de sua validade.

Demonstração do Lema 9. Dividiremos a demonstração do Lema 9 em três afirmações que serão listadas a seguir. Logo em seguida argumentaremos que destas afirmações segue a afirmação do Lema 9.

1. Se $\bar{A}_{0} \geq \bar{A}_{*}$ e $H_{0} \leq h_{*}$, existe $l \in \mathbb{N}$, tal que

$$
H_{j} \leq h_{*}, \quad \bar{A}_{j}>0, \quad 0 \leq j \leq l \text { e } H_{l+1}>h_{*}
$$

2. Se $\bar{A}_{0}>\bar{A}_{*}$ e $H_{1}>h_{*}$, existe $i \in \mathbb{N}, i \geq 1$, tal que

$$
\bar{A}_{j}>0, \quad 1 \leq j \leq i-1, \quad \bar{A}_{i} \leq 0 ; \quad H_{i}>h_{*}
$$

3. Se $\bar{A}_{0} \leq 0$ e $H_{0}>h_{*}$, existem $\lambda_{c} \in \mathbb{R}, \lambda_{c}>0$, e $k \in \mathbb{N}, k \geq 1$, tais que

$$
\bar{A}_{j}<0, \quad 1 \leq j \leq k, \quad \bar{A}_{k}<-\bar{A}_{*}
$$

A partir da validade das afirmações (1), (2) e (3), podemos facilmente concluir a afirmação do Lema 9. Note que cada seqüência de cada afirmação possui uma condição final que inclui a condição inicial da sequiência seguinte. Ou seja, se $H_{0} \leq h_{*}$, concluímos por (1), (2) e (3) que existem $l, i$ e $k$ finitos, tais que (6.39) e (6.40) valham para $u:=l+i$ e $v:=l+i+k$. Se (já logo de início) valer $H_{0}>h_{*}$, concluímos por (2) e (3) a validade de (6.39) e (6.40) onde, neste caso então, $u:=i$ e $v:=i+k$.

Posto isto, checaremos a seguir tão somente a validade das afirmações (1), (2) e (3) isoladamente. 
Demonstração da afirmação (1). Sejam $\bar{A}_{0} \geq \bar{A}_{*}$ e $l \in \mathbb{N} \cup\{\infty\}$ ( $l$ fixo, porém arbitrário, podendo inclusive ser infinito). Inicialmente, provaremos a seguinte implicação:

$$
H_{t} \leq h_{*}, \quad 0 \leq t<l+1 \quad \Rightarrow \quad \bar{A}_{t}>\bar{A}_{*}, 0 \leq t<l+1
$$

Assumiremos que a afirmação do lado esquerdo da implicação (6.45) seja verdadeira e provaremos, por indução em $t$, a afirmação do lado direito da implicação (6.45).

Para $t=0$ esta afirmação está garantida pela condição inicial $\bar{A}_{0} \geq \bar{A}_{*}$. Vamos supor então que a afirmação valha para algum $t$, onde $0 \leq t<l$, i.e., $\bar{A}_{t} \geq \bar{A}_{*}$ e vamos provar $\bar{A}_{t+1} \geq \bar{A}_{*}$.

Como $\bar{A}_{t+1}=g\left(\bar{A}_{t} ; H_{t+1}\right)$ e como, por hipótese, $\bar{A}_{t} \geq \bar{A}_{*}$ e $H_{t+1} \leq h_{*}$, bașta provar que

$$
\bar{A} \geq \bar{A}_{*}, h \leq h_{*} \quad \Rightarrow \quad g(\bar{A} ; h) \geq \bar{A}_{*}
$$

Demonstração de (6.46). De acordo com a Proposição $2, g(\cdot ; h)$ possui uma solução $\bar{A}_{+}(h) \geq \bar{A}_{*}$ sempre que $h \leq h_{*}$. Portanto, se $\bar{A} \geq \bar{A}_{+}(h)$, segue $g(\bar{A} ; h) \geq g\left(\bar{A}_{+}(h) ; h\right)=$ $\bar{A}_{+}(h)>\bar{A}_{*}$ de onde concluímos (6.46). Observamos que a primeira das duas últimas desigualdades segue do fato de $g(\cdot ; h)$ ser crescente.

Por outro lado, as desigualdades (6.6), (6.8) e (6.9) garantem que $g(\bar{A} ; h) \geq \bar{A}$ se $\bar{A}_{*} \leq$ $\bar{A} \leq \bar{A}_{+}(h)$. Portanto, a afirmação (6.46) vale também para o caso em que $\bar{A} \leq \bar{A}_{+}(h)$. Ou seja, provamos (6.46). Assim, acabamos de mostrar (6.45) também.

Vamos agora mostrar que, se $\bar{A}_{0}>\bar{A}_{*}$ e $H_{0} \leq h_{*}$, então existe um primeiro momento $l+1 \in \mathbb{N}$, tal que $H_{t+1}>H_{*}$. O argumento segue por contradição. Caso não exista $l+1$, i.e., caso $H_{t} \leq h_{*}, \forall t \in \mathbb{N}$, então teríamos, de acordo com (6.45), $\bar{A}_{t} \geq \bar{A}_{*}, \forall t \in \mathbb{N}$ e conseqüentemente $\bar{A}_{t}=H_{0}+\lambda \sum_{t=0}^{t-1} \bar{A}_{t} \geq H_{0}+t \bar{A}_{*} \uparrow \infty$. Este fato contradiz $H_{t} \leq h_{*}$, $\forall t \in \mathbb{N}$. Provamos que existe um momento $l+1 \in \mathbb{N}$ em que $H_{l+1}>0$.

Podemos assumir então, sem perda de generalidade, que $l+1$ é o primeiro momento em que $H_{l+1}>0$, i.e., $H_{t} \leq h_{*}, 0 \leq t<l+1$. Como (6.45) já foi provado, concluímos $\bar{A}_{t}>t, 0 \leq t<l+1$.

Acabamos de provar a afirmacão (1).

Demonstração da afirmação (2). Mostraremos que, se não valer a afirmação (2), obteremos uma contradição. Mais precisamente, mostraremos inicialmente que, se não valer (2), 
então existe condições iniciais $\bar{A}_{0}>\bar{A}_{*}$ e $H_{1}>h_{*}$, tais que

$$
\bar{A}_{t}>0, \quad H_{t} \geq H_{1}\left(>h_{*}\right) \quad t=0,1,2, \ldots
$$

A partir desse fato, obteremos a contradição.

Suponhamos que a afirmação (2) não valha. Neste caso, existe $\bar{A}_{0}>\bar{A}_{*}$ e $H_{1}>h_{*}$, tais que, para cada $t=1,2, \ldots$, ou $\bar{A}_{t}>0$ ou $H_{t} \leq h_{*}$. Vamos provar então que para estas condições iniciais vale a afirmação (6.47).

Demonstração. Por indução. Para $t=1$, segue pela condição inicial que $H_{1}>h_{*}$. Portanto, como ou $\bar{A}_{1}>0$ ou $H_{1} \leq h_{*}$, temos necessariamente $\bar{A}_{1}>0$. Suponhamos que valem $H_{t}>H_{1}$ e $\bar{A}_{t}>0$ para algum $t \geq 1$. Então, temos $H_{t+1}=H_{t}+\lambda \bar{A}_{t}$. Usando a hipótese de indução, segue $H_{t+1} \geq H_{1}$. Novamente, como deve valer $\bar{A}_{t+1}>0$ ou $H_{t+1} \leq h_{*}<H_{1}$, segue necessariamente $\bar{A}_{t+1}>0$. Acabamos de provar (6.47) sob a hipótese de que a afirmação (2) não é verdadeira.

Vamos mostrar agora que, para toda órbita $\left(H_{t}, \bar{A}_{t}\right)_{t \geq 0}$, onde $H_{t} \geq H_{1} \geq h_{*}, t=$ $1,2, \ldots$, existe um $i \in \mathbb{N}$, tal que $\bar{A}_{i}<0$. Este fato contradiz (6.47) e prova, portanto, a afirmação (2). Mais precisamente, provaremos que, para qualquer $\bar{A}_{0} \in[-1,1]$, vale a implicação

$$
H_{t}>H_{1}>h_{*}, \quad t=1,2, \ldots \Rightarrow \bar{A}_{t} \leq g^{(t)}\left(\bar{A}_{0} ; H_{1}\right), \quad t=1,2, \ldots
$$

onde usamos novamente a notação $g^{(t)}\left(\bar{A}_{0} ; H_{1}\right)$ para designar a t-ésima interação de $g\left(\cdot ; H_{1}\right)$ em $\bar{A}_{0}$, i.e., $g^{(t)}\left(\bar{A}_{0} ; H_{1}\right)=g^{(t)}\left(g^{(t-1)}\left(\bar{A}_{0} ; H_{1}\right) ; H_{1}\right)$, onde $g^{(1)}\left(\bar{A}_{0} ; H_{1}\right)=g\left(\bar{A}_{0} ; H_{1}\right)$. Como $H_{1}>h_{*}$, segue da Proposição 2 que $g\left(\cdot ; H_{1}\right)$ possui um único ponto fixo $\bar{A}_{-}\left(H_{1}\right)$, onde $\bar{A}_{-}\left(H_{1}\right)<-\bar{A}_{*}<0$. Também pela Proposição 2 , segue que $g^{(t)}\left(\bar{A}_{0} ; H_{1}\right)$ converge para o ponto fixo negativo $\bar{A}_{-}\left(H_{1}\right)$. Dessa convergência e da desigualdade do lado direito da implicação (6.48), obtemos imediatamente que existe um $i \in \mathbb{N}$, tal que $\bar{A}_{i}<0$. Este fato contradiz (6.47) e prova a afirmação (2). Vamos provar, portanto, (6.48).

Demonstração. Por indução. Para $t=1$ segue da definição do sistema dinâmico que $\bar{A}_{1}=g\left(\bar{A}_{0} ; H_{1}\right)=g^{(1)}\left(\bar{A}_{0} ; H_{1}\right)$. Vamos supor que a desigualdade do lado direto da implicação (6.48) vale para $t \geq 1$, então

$$
\bar{A}_{t+1}=g\left(\bar{A}_{t} ; H_{t+1}\right) \leq g\left(\bar{A}_{t} ; H_{1}\right) \leq g\left(g^{(t)}\left(\bar{A}_{0} ; H_{1}\right) ; H_{1}\right)=g^{(t+1)}\left(\bar{A}_{0} ; H_{1}\right)
$$


A primeira desigualdade de (6.49) segue do fato de $g\left(\bar{A}_{t} ; \cdot\right)$ ser decrescente e $H_{t+1} \geq$ $H_{1}$. A segunda desigualdade de (6.49) segue do fato de $g\left(\cdot ; H_{1}\right)$ ser crescente e $\bar{A}_{t} \leq$ $g^{(t)}\left(\bar{A}_{0} ; H_{1}\right)$. Esta última desigualdade que acabamos de afirmar constitui a hipótese de indução. Acabamos, portanto, de demonstrar a afirmação (2).

Demonstração da afirmação (3). Para a demonstração da afirmação (3) faremos uso da seqüência decrescente $g^{(t)}\left(0 ; h_{*} / 2\right)$ e de sua convergência monótona para o ponto fixo negativo $\bar{A}_{-}\left(h_{*} / 2\right)$ de $g\left(\cdot ; h_{*} / 2\right)$. Novamente adotamos a notação $g^{(t)}\left(0 ; h_{*} / 2\right)$ para designar a $t$-ésima interação de $g\left(\cdot ; h_{*} / 2\right)$ em 0 , i.e., $g^{(t)}\left(0 ; h_{*} / 2\right)=g^{(t)}\left(g^{(t-1)}\left(0 ; h_{*} / 2\right) ; h_{*} / 2\right)$, onde $g^{(1)}\left(0 ; h_{*} / 2\right)=g\left(0 ; h_{*} / 2\right)$.

Lembramos que, pela Proposição 2, o menor ponto fixo de $g(\cdot ; h)$, denotado por $\bar{A}_{-}(h)$, está sempre abaixo de $-\bar{A}_{*}$ para quaisquer valores de $h$ satisfazendo $|h|<h_{*}$. Em particular, vale $\bar{A}_{-}\left(h_{*} / 2\right)<-\bar{A}_{*}$.

A demonstração da afirmação (3) consistirá em provar a seguinte afimação: para todo $k \geq 1, A_{0} \leq 0$ e $H_{0}>h_{*}$, vale

$$
\lambda \leq \lambda(k):=\frac{h_{*}}{2(k+1)} \quad \Rightarrow \bar{A}_{t} \leq g^{(t)}\left(0 ; h_{*} / 2\right) \quad \forall t \in\{1, \ldots k\}
$$

Note que a afirmação (3) segue da implicação (6.50), onde $\lambda_{c}$ é definido por

$$
\lambda_{c}:=\lambda(k)=\frac{h_{*}}{2(k+1)}, \quad \text { para um } k>0: g^{(k)}\left(0 ; h_{*} / 2\right)<-\bar{A}_{*}
$$

Para concluir a afirmação (3) a partir da implicação (6.50), basta verificar que:

(a) Zero pertence ao domínio de atração de $\bar{A}_{-}\left(h_{*} / 2\right)$, i.e., $g^{(t)}\left(0 ; h_{*} / 2\right)$ converge para $\bar{A}_{-}\left(h_{*} / 2\right)$ (e não para $\bar{A}\left(h_{*} / 2\right)$ e nem para $\left.\bar{A}_{+}\left(h_{*} / 2\right)\right)$; e

(b) $g^{(t)}\left(0 ; h_{*} / 2\right) \downarrow \bar{A}_{-}\left(h_{*} / 2\right)<-\bar{A}_{*}$, i.e., a convergência de $g^{(t)}\left(0 ; h_{*} / 2\right)$ para $\bar{A}_{-}\left(h_{*} / 2\right)$ é monótona.

No parágrafo seguinte verificaremos a observação $(a)$; e no parágrafo subseqüente verificaremos a observação $(b)$. Após a verificação destas duas observações, mostraremos a validade da implicação (6.50).

Verificação da observação (a). Lembramos que, para $|h|<h_{*}$, a função $g(\cdot ; h)$ possui três pontos fixos, $\bar{A}_{-}(h), \bar{A}(h)$ e $\bar{A}_{+}(h)$, dos quais $\bar{A}_{-}(h)$ e $\bar{A}_{+}(h)$ são atratores. Quando 
$|h|<h_{*}$, o domínio de atração de $\bar{A}_{-}(h)$ é dado pelo intervalo $[-1, \bar{A}(h))$. Precisamos checar, portanto, se $0<\bar{A}\left(h_{*} / 2\right)$. Pelo item 2c do Lema 3 , segue que o ponto fixo intermediário $\bar{A}(h)$ é uma função ímpar e estritamente crescente de $h$ para $h \in\left(-h_{*}, h_{*}\right)$. Como $0<h_{*} / 2<h_{*}$, segue, portanto, $0=\bar{A}(0)<\bar{A}\left(h_{*} / 2\right)$, i.e., 0 pertence ao domínio de atração de $\bar{A}_{-}\left(h_{*} / 2\right)$.

Verificação da observação (b). Lembramos ainda que, segundo a demonstração da Proposição $2, g^{(t)}\left(\bar{A}_{0} ; h\right) \downarrow \bar{A}_{-}(h)$, sempre que $|h|<h_{*}$ e $\bar{A}_{-}(h)<\bar{A}_{0}<\bar{A}_{+}(h)$. Como, em particular, $\bar{A}_{-}\left(h_{*} / 2\right)<-\bar{A}_{*}<0<\bar{A}_{+}\left(h_{*} / 2\right)$, segue $g^{(t)}\left(0 ; h_{*} / 2\right) \downarrow \bar{A}_{-}\left(h_{*} / 2\right)<-\bar{A}_{*}$, como havíamos afirmado logo após (6.50).

Demonstraremos agora a implicação (6.50) sob a condição de que $\bar{A}_{0} \leq 0$ e $H_{0} \geq h_{*}$. Para tanto, vamos inicialmente estimar $H_{t}$ para $0 \leq t \leq k, \lambda<\lambda(k)$ e $H_{0}>h_{*}$. Esta estimativa segue abaixo.

$$
\begin{aligned}
H_{t+1} & =H_{0}+\lambda \sum_{j=0}^{t} \bar{A}_{j} \\
& \geq H_{0}-\lambda(t+1) \\
& \geq H_{0}-\lambda(k)(k+1)=H_{0}-\left[h_{*} / 2(k+1)\right](k+1)=H_{0}-h_{*} / 2 \\
& \geq h_{*} / 2
\end{aligned}
$$

A desigualdade (6.52) vem de $\bar{A}_{t} \geq-1, \forall t \geq 0$.

Vamos agora concluir a prova de (6.50) por indução em $t=0,1,2, \ldots \mathbb{N}$ para $k$ constante, porém arbitrário $(k \geq 1)$.

Início da indução $(t=0)$. Vamos checar inicialmente a validade de (6.50) para $t=0$. Pela condição inicial, temos $\bar{A}_{0} \leq 0$; e, pela desigualdade (6.53), temos $H_{1}>h_{*} / 2$. Portanto, para $t=0$, segue

$$
\bar{A}_{1}=g\left(\bar{A}_{0} ; H_{1}\right) \leq g\left(\bar{A}_{0} ; h_{*} / 2\right) \leq g\left(0 ; h_{*} / 2\right)
$$

A primeira desigualdade de (6.54) segue do fato de $g\left(\bar{A}_{0} ; \cdot\right)$ ser decrescente e $H_{1} \geq h_{*} / 2$. A segunda desigualdade de (6.54) segue do fato de $g\left(\cdot ; h_{*} / 2\right)$ ser crescente e $\bar{A}_{0} \leq 0$.

Passo da indução $(t \curvearrowright t+1)$. Supondo agora que a desigualdade do lado direito da implicação (6.50) vale para $0 \leq t<k$, vamos concluir que esta mesma desigualdade vale 
para $t+1$. Sob a hipótese de indução, temos

$$
\bar{A}_{t+1}=g\left(\bar{A}_{t} ; H_{t+1}\right) \leq g\left(\bar{A}_{t} ; h_{*} / 2\right) \leq g\left(g^{(t)}\left(0 ; h_{*} / 2\right) ; h_{*} / 2\right)=g^{(t+1)}\left(0 ; h_{*} / 2\right)
$$

A primeira desigualdade de (6.55) segue do fato de $g\left(\bar{A}_{t} ; \cdot\right)$ ser decrescente e $H_{t+1} \geq h_{*} / 2$ por (6.53). A segunda desigualdade de (6.55) segue do fato de $g\left(\cdot ; h_{*} / 2\right)$ ser crescente e $\bar{A}_{t} \leq g^{(t)}\left(0 ; h_{*} / 2\right)$. Esta última desigualdade que acabamos de afirmar é a hipótese de indução.

Acabamos de demonstrar a afirmação (3). O término da demonstração da afirmação (3) fecha a demonstração do Lema 9. 


\section{Referências Bibliográficas}

[1] W. B. Arthur. Inductive reasoning and bounded rationality (the el farol problem). Amer. Econ. Review (P.apers and Proceedings), 84:406, 1994.

[2] W. B. Artthur. Increasing returns, competing technologies and lock-in by historical small events: The dynamics of allocation under increasing returns to scale. Economic Journal, 99:116-131, 1989.

[3] A. Banerjee. A simple model of herd behavior. Quarterly Journal of Economics, CVII:797-818, 1992.

[4] F. P. A. Prado; P. Tommasini; V. Belitsky. Night clubs pricing. Universidade de São Paulo, Working in Progress, 2004.

[5] L. Blume. The statistical mechanics of strategic interaction. Games and Economic Behavior, 5:387-424, 1993.

[6] W. Brock. Pathways to randomness in the economy: Emergent nonlinearity and chaos in economics and finance. Estudios Economicos, 8(1):3-55, 1993.

[7] S. Durlauf. Nonergodic economic growth. Review of Economic Stuedies, 60:349-366, 1993.

[8] W. Brock; S. Durlauf. Discrete choice with social interactions. Working paper, University of Wisconsin at Madison, 1995.

[9] W. Brock; S. Durlauf. Interactions Based Models. Handbook of Econometrics. Ed. by J. Heckman and E. Learner, Amsterdam: North-Holland, to appear. 
[10] G. Ellison. Learning, local interaction and coordination. Econometrica, 61:10471072, 1993.

[11] H. Föllmer. Random economies with many interacting agents. Journal of Mathematical Economics, 1:51-62, 1974.

[12] G. Ellison; D. Fudemberg. Rules of thumb for social learning. Jornal of Political Economy, 101:612-644, 1993.

[13] K. Kacperski; J. A. Holyst. Opinion formation model with strong leader and external impact: A mean field approach. Physica A, 269:511-526, 1999.

[14] K. Kacperski; J. A. Holyst. Phase transitions as a persistent feature of groups with leaders in models of opinion formation. Physica A, 287:631-643, 2000.

[15] T. Kaizoji. Speculative bubbles and crashes in stock markets: An interacting-agent model of speculative activity. Physica A, 287:493-506, 2000.

[16] A. Kirman. Ants, rationality, and recruitment. Quarterly Journal of Economics, 93:137-156, 1993.

[17] Thomas M. Liggett. Interacting Particle System. Springer-Verlag, 1985.

[18] F. Gul; R. Lundholm. Endogenous timing and the ckystering of agents' decisions. Journal of Political Economy, 103:619-638, 1995.

[19] T. Lux. Herd behavior, bubbles and crashes. The Economic Jornal, 105:881-896, 1995.

[20] G. Becker; K. M. Murphy. Social Economics: Market Behavior in a Social Environment. Cambridge: Belknap-Harvard University Press, 2000.

[21] W. Pesendorfer. Design innovation and fashion cycles. The America Economic Review, 85(4):771-792, 1995.

[22] E. Glaeser; B. Sacerdote; J. Scheinkman. Crime and social interactions. Quarterly Journal of Economics, CXI:507-548, 1996. 
[23] E. Glaeser; J. Scheinkman. Non-market interactions. Working paper, Dep. of Economics, Princeton University, 2000.

[24] E. Glaeser; J. Scheinkman. Measuring Social Interactions. Social Dynamics. Ed. by S. Durlauf and P. Young., Cambridge: MIT Press, 2001.

[25] V. Madrigal; J. Scheinkman. Price crashes, information aggregation, and marketmaking. Jornal of Economic Theory, 75:16-63, 1997.

[26] E. J. Neves; R. H. Schonmann. Critical droplets and metastability for a glauber dynamics at very low temperature. Communications in Mathematical Physics, 137:209230, 1991.

[27] E. J. Neves; R. H. Schonmann. Behavior of droplets for a class of glauber dynamics at very low termperature. Probability Theory and Related Fields, 91:331-354, 1992.

[28] A. Aleksiejuk; J. A. Holyst; Dietrich Stauffer. Ferromagnetic phase transiction in barabási-albert networks. Physica A, 310:260-267, 2002.

[29] G. Topa. Social interactions, local spillovers and unemployment. Working paper. New York University, 1997.

[30] S. Bickhchandani; D. Hirshleifer; I. Welch. A theory of fads, fashion, custom, and cultural exchange as information cascades. Journal of Political Economy, 100:9921026, 1992.

[31] K. D. West. Bubbles, fads, and stock price volatility tests: A partial evaluation. Journal of Finance, 43:639-660, 1988.

[32] P. Bak; K. Chen; J. Sheinkman; M. Woodford. Aggregate fluctuations from independent setorial shocks: Self-organized criticality in a model or production and inventory dynamics. Ricerche Economiche, 47:3-30, 1993.

[33] J. Scheinkman; W. Xiong. Overconfidence and speculative bubbles. Working paper, Dep. of Economics, Princeton University, 2002. 
[34] D. Challet; M. Marsili; Y.-C.Zhang. From minority games to real markets. condmat/0011042, 1, 2000.

[35] H. P. Young. The evolution of conventions. Econometrica, 61:57-84, 1993.

[36] D. Challet; M. Marsili; R. Zecchina. Modeling market mechanism with minority game. cond-mat/9909265vl, page 1999, 2000.

[37] D. Challet; M. Marsili; R. Zecchina. Statistical mechanics of heterogeneous agents. Phys. Rev. Lett., 84:1824, 2000.

[38] M. Marsili; Challet; R. Zecchina. Exact solution of a modified el farol's bar problem. Physica A, 280:522, 2000.

[39] A. Dembo; O. Zeitouni. Large Deviations Techinques and Applications. Jones and Bartlett Publishers, London W6 7RS, England, 1993.

[40] D. Challet; Y.-C. Zhang. Emergence of cooperation and organization in an evolutionary game. Physica A, 246:407, 1997.

[41] D. Challet; Y.-C. Zhang. Phase transition and symmetry breaking in the minority game. Phys. Rev. E, 60:R6271, 1997. 